\title{
Observation of s-Channel Single Top Quark Production at the Tevatron
}

\author{
Matteo Cremonesi \\ St. Catherine's College, Oxford
}

Thesis submitted in fulfilment of the requirements for the degree of Doctor of Philosophy at the University of Oxford

Michaelmas Term, 2014 


\section{Declaration}

This dissertation is the result of my own work, except where explicit reference is made to the work of others, and has not been submitted for another qualification to this or any other university. This dissertation does not exceed the page limit for the respective Degree Committee.

Matteo Cremonesi

\section{Personal Contribution}

Due to the large nature of particle physics experimental collaborations, the work in this thesis relies on the results of a large number of individual researchers and analysis subgroups. A major result of the work presented in this thesis was the observation of the $s$-channel single top quark production. The author contributed to this result as the main analyzer of one of the three analyses that were included in the final Tevatron combination. The author was also responsible for both the CDF and the Tevatron combination. The work presented in this thesis is reported in the papers listed below:

- Observation of $s$-Channel Production of Single Top Quarks at the Tevatron Published in PRL

T. Aaltonen et al. (CDF and D0 Collaborations), Phys. Rev. Lett. 112, 231803 (2014). Selected as PRL Editors' Suggestion

- Search for $s$-Channel Single-Top-Quark Production in Events with Missing Energy Plus Jets in $p \bar{p}$ Collisions at $\sqrt{s}=1.96 \mathrm{TeV}$

Published in PRL

T. Aaltonen et al. (CDF Collaboration), Phys. Rev. Lett. 112, 231805 (2014).

- Measurement of the single-top-quark cross section and the CKM matrix element $\left|V_{t b}\right|$ at the Tevatron

CDF and D0 Collaborations, Tevatron Electroweak Working Group, To be submitted soon. 
- Updated measurement of the single top quark production cross section and $\left|V_{t b}\right|$ in the missing transverse energy plus jets topology in $p \bar{p}$ collisions at $\sqrt{s}=\mathbf{1 . 9 6}$ $\mathrm{TeV}$

Submitted to PRL

T. Aaltonen et al. (CDF Collaboration), arXiv:1410.4909.

\section{- Search for New Resonances Decaying to a Top and a Bottom Quark at CDF}

T. Aaltonen et al. (CDF Collaboration), To be submitted soon.

Leading contributions by the author presented in this thesis are outlined below, subdivided by thesis chapter.

\section{Chapter 4: Model of the Selected Data Sample}

The author has performed a study on the dependence of the $k$ factor that corrects the $V+\mathrm{HF}$ prediction on the number of $b$ tags. This study led to two conclusions: the rate of $V+\mathrm{HF}$ is enhanced in the single-tag category by $V+$ two-heavy-flavour-quark events in which both the jets originating from the two HF quarks are clustered into a single jet; $V$ +two-heavy-flavour-quark events with one heavy flavour jet and one mistagged light-flavour jet originating from initial or final state radiation were not properly taken into account. The study resulted in an improved modelling of the kinematic variables considered in the analysis and in the systematic uncertainty.

\section{Chapter 6: Observation of s-Channel Single Top Quark Production}

The author has been the main analyzer of the $E_{T} b \bar{b}$ analysis, as well as both the CDF and the Tevatron combination, presented in this chapter. The original contribution consists of the development of the staged multivariate technique adopted in the $\mathbb{E}_{T} b \bar{b}$ analysis, starting from the definition of the strategy and the selection of the kinematic variables used in the training, to the derivation of the different neural networks employed in this analysis and their validation. The statistical analysis that uses these discriminants to extract the signal was performed by the author. The author wrote the framework for the statistical combination of the two CDF searches for s-channel single top quark production. The author participated actively in the discussion on the correlation of uncertainties among the two searches to be treated as nuisance parameters. The author had responsibility for the final Tevatron combination that led to the first observation of the process, setting up the code for the statistical combination, iterating and cross-checking with the D0 experiment and extracting the cross section of the process. 


\section{Chapter 7: Search for New Resonances Decaying to a Top and a Bottom Quark}

The author was the main analyzer of the $W^{\prime}$ search presented in this chapter. The original contributions consist of the optimization of the event selection, including events with reconstructed leptons; the development of the staged multivariate technique, starting from the definition of the strategy and the selection of the kinematic variables used in the training to the derivation of the different neural networks employed in this analysis and their validation. The statistical analysis that uses these discriminants to extract the limits was performed by the author.

\section{Appendix A: Measurement of the Single Top Quark Production Cross Section and the CKM Matrix Element $\left|V_{t b}\right|$ at the Tevatron}

The author has been the main analyzer of the $E_{T} b \bar{b}$ analysis, as well as both the CDF and the Tevatron combination, presented in this chapter. The original contribution consists of the development of the staged multivariate technique adopted in the $\mathbb{E}_{T} b \bar{b}$ analysis, starting from the definition of the strategy and the selection of the kinematic variables used in the training to the derivation of the different neural networks employed in this analysis and their validation. The statistical analysis that uses these discriminants to extract the signal was performed by the author. The author wrote the framework for the statistical combination of the two CDF analyses on single top quark production. The author participated actively in the discussion on the correlation of uncertainties among the two searches to be treated as nuisance parameters. The author had responsibility for the final Tevatron combination, setting up the code for the statistical combination, iterating and cross-checking with the D0 experiment and extracting the cross section of the process. 


\section{Abstract}

Testing the Standard Model (SM) and looking for new phenomena have been the focus of generations of particle physicists in the last decades. Following this spirit, this thesis presents two searches.

The first is the search for single top quark production from the exchange of an s-channel virtual $W$ boson using events with an imbalance in the total transverse energy, $b$-tagged jets, and no identified leptons. Assuming the electroweak production of top quarks of mass $172.5 \mathrm{GeV} / c^{2}$ in the $s$-channel, a cross section of $1.12_{-0.57}^{+0.61}$ (stat+syst) pb, with a significance of 1.9 standard deviations, is measured. This measurement is combined with the result obtained from events with an imbalance in total transverse momentum, $b$-tagged jets, and exactly one identified lepton, yielding a cross section of $1.36_{-0.32}^{+0.37}$ (stat+syst) pb, with a significance of 4.2 standard deviations. The first observation of single-top-quark production in the $s$ channel through the combination of the CDF and D0 measurements is also reported. The measured cross section is $\sigma_{s}=1.29_{-0.24}^{+0.26} \mathrm{pb}$. The probability of observing a statistical fluctuation of the background to a cross section of the observed size or larger is $1.8 \times 10^{-10}$, corresponding to a significance of 6.3 standard deviation.

The second is the search for $W^{\prime}$-like resonances decaying to $t b$. No significant excess above the SM prediction is found. Using a benchmark $W^{\prime} \rightarrow t b$ left-right symmetric model, 95\% C.L. mass-dependent upper limits are placed on the $W^{\prime}$ boson production cross section times branching ratio to $t b$. Assuming a $W^{\prime}$ boson with SM-like couplings and allowed (forbidden) decay to leptons, $W^{\prime} \rightarrow t b$ is excluded with 95\% C.L. for $W^{\prime}$ boson masses below 860 (880) $\mathrm{GeV} / c^{2}$. Relaxing the hypothesis on SM-like couplings, we exclude $W^{\prime}$ boson coupling strength values as a function of the $W^{\prime}$ boson mass above $10 \%$ of the SM coupling strength for $M_{W^{\prime}}=300 \mathrm{GeV} / c^{2}$. The constraints obtained with the present analysis are the most stringent for charged resonance masses below $570 \mathrm{GeV} / c^{2}$ decaying to a top and a bottom quark. 
To my parents. 


\section{Acknowledgements}

I would like to thank all the people who contributed to the completion of this thesis work.

My advisor in Oxford, Dr Chris Hays, for his presence over these years as a DPhil student in Oxford, for the precise and detailed correction of this thesis, for being constantly in touch with me, for his crucial insight.

My advisor at UChicago, Prof Young-Kee Kim, for the invaluable support she gave me in solving both technical and personal issues, for giving me the possibility to develop skills that will be essential to my future career, for being for me an example that made me grow as a physicist and as a man.

My Fermilab supervisor, Dr Tom Junk, for the patience and the time he spent with me. I learned a lot from him, from his deep knowledge and wisdom.

Professor Melissa Franklin, for her precious advice and her availability. I enjoyed so much the conversation we had in Boston, they helped me building a better comprehension of the field.

CDF spokespersons and conveners, Dr Costas Vellidis, Dr Luciano Ristori, Prof Dave Toback and Dr Jon Wilson, for being constantly present in every aspect of my life at CDF, for giving me the opportunity to take responsibilities and risks, for trusting me.

Professor Giorgio Bellettini, for being an extremely passionate and attentive mentor.

Dr Fabrizio Margaroli, for always giving me the right suggestion. The ideas that I developed in this thesis work come from him.

Dr Qiuguang Liu, for teaching me the analysis code, for being always available every time a problem arose, for being a good friend.

Prof Craig Group, Dr Hao Liu, Prof Reinhard Schwienhorst and Prof Christian Schwanenberger, for the fruitful, successful and enjoyable collaboration. 
Dr Eric James, for the effort he put in showing me how to write a good paper and how to prepare and give a good seminar.

And my mother and my father, for being a unique source of strength, for being my family. 


\section{Contents}

1 Theoretical Background $\quad 1$

1.1 The Standard Model . . . . . . . . . . . . . . . . . . . . . . . . . 1

1.1.1 Fundamental Forces and Particles . . . . . . . . . . . . . . . 2

1.1.2 The Electroweak Model and the Higgs Mechanism . . . . . . . . . . 3

1.1.3 Quantum Chromodynamics ................. 5

1.2 The Top Quark . . . . . . . . . . . . . . . . . . . . . . . 6

1.2.1 Top-Pair Production Through the Strong Interaction . . . . . . . . . . 6

1.2.2 Electroweak Single Top Quark Production . . . . . . . . . . . . . . . 8

1.2 .3 Top-Quark Decay . . . . . . . . . . . . . . . . . . . . . . . . . . . . . . . . . . 11

1.3 Searching for a New Particle . . . . . . . . . . . . . . . . . . . 11

1.3.1 Features of a $W^{\prime}$ Boson . . . . . . . . . . . . . . 13

2 The Experiment $\quad 15$

2.1 The Tevatron Collider . . . . . . . . . . . . . . . . . . . . . . 15

2.1 .1 The Proton Source . . . . . . . . . . . . . . . . . . . 15

2.1 .2 The Antiproton Source . . . . . . . . . . . . . . . . 16

2.1 .3 The Tevatron Ring . . . . . . . . . . . . . . . . . . . 17

2.1.4 Luminosity and Tevatron Performance . . . . . . . . . . . . 18

2.2 The CDF II Detector . . . . . . . . . . . . . . . . . . . . . . 18

2.2.1 Coordinates System and Conventions at CDF . . . . . . . . . . 20

2.3 The Tracking System . . . . . . . . . . . . . . . . . . . . . . 21

2.3.1 The Silicon Tracker . . . . . . . . . . . . . . . . . . . 23

2.3.2 Central Outer Tracker . . . . . . . . . . . . . . . . 24

2.3 .3 Time of Flight . . . . . . . . . . . . . . . . . . . 26

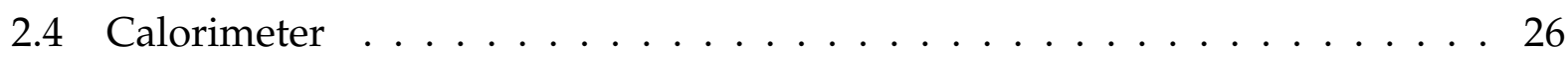

2.4 .1 The Central Calorimeter . . . . . . . . . . . . . . . . . . 27

2.4 .2 The Plug Calorimeter . . . . . . . . . . . . . . . . . . 29

2.5 The Muon Chambers . . . . . . . . . . . . . . . . . . . . . . . . . . . . 29

2.6 CLC Detector . . . . . . . . . . . . . . . . . . . . 31

2.7 The CDF Trigger System . . . . . . . . . . . . . . . . . . . . . . . . . . . . . . . . .

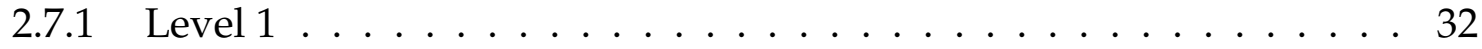

2.7 .2 Level $2 \ldots \ldots \ldots \ldots \ldots \ldots$

2.7 .3 Level $3 \ldots \ldots \ldots \ldots \ldots$ 
3 Reconstruction of Physics Objects $\quad 35$

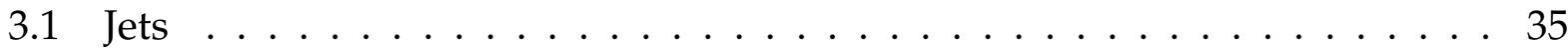

3.1 .1 Jet Clustering . . . . . . . . . . . . . . . 35

3.1 .2 Jet Energy Corrections . . . . . . . . . . . . . . . . . . . 37

3.1 .3 The H1 Algorithm . . . . . . . . . . . . . . . . . . 38

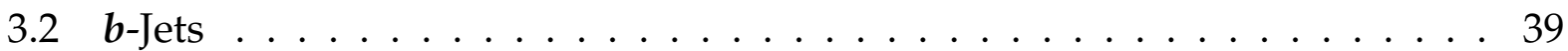

3.2.1 The SECVTX Algorithm . . . . . . . . . . . . . . . 40

3.2 .2 The JETPROB Algorithm . . . . . . . . . . . . . . . . 41

3.2 .3 The HOBIT Algorithm . . . . . . . . . . . . . . . 43

3.3 Missing Transverse Energy . . . . . . . . . . . . . . . . . . . . 44

3.4 Lepton Identification . . . . . . . . . . . . . . . . . . . . . 48

3.4 .1 Electron Identification . . . . . . . . . . . . . . . . . 48

3.4 .2 Muon Identification . . . . . . . . . . . . . . . . . . . . 49

4 Model of the Selected Data Sample $\quad 50$

4.1 Data Sample . . . . . . . . . . . . . . . . . . . . . . . 50

4.2 Event Selection . . . . . . . . . . . . . . . . . . . 50

4.2.1 Online Requirements and Trigger Efficiency . . . . . . . . . . . . . 50

4.2.2 Offline Requirements and Control Regions . . . . . . . . . . . . . 53

4.3 Background Model . . . . . . . . . . . . . . . . . . 55

4.3.1 EWK Heavy-Flavour Background Model . . . . . . . . . . . . . 56

4.3 .2 EWK Light-Flavour Mistag Model . . . . . . . . . . . . . . . . . . . . 59

4.3 .3 QCD Multijet Model . . . . . . . . . . . . . . . . . . . . 62

4.4 Signal Model . . . . . . . . . . . . . . . . . . . 65

5 Statistical Interpretation $\quad 66$

5.1 Bayesian Approach . . . . . . . . . . . . . . . . . 67

5.1 .1 Markov Chain Integration . . . . . . . . . . . . . . . . . 68

5.1.2 Combination of Multiple Bins and Channels . . . . . . . . . . . . 69

5.2 Upper Limit and Cross Section Measurement . . . . . . . . . . . . . . . 70

5.2.1 Expected and Observed Limits and Cross Sections . . . . . . . . . . . 71

5.3 Significance Calculation . . . . . . . . . . . . . . . 71

6 Observation of $s$-Channel Single Top Quark Production $\quad 73$

6.1 Search for $s$-Channel Single Top Quark Production in the $\boldsymbol{E}_{T} \boldsymbol{b} \overline{\boldsymbol{b}}$ Sample . . 74

6.1.1 Definition of the Analysis Subsamples . . . . . . . . . . . . . . 74

6.1 .2 Multivariate Discriminants . . . . . . . . . . . . . . . 75

6.1.3 Cross Section Extraction and Significance Calculation . . . . . . . . . 78

6.2 CDF Combination . . . . . . . . . . . . . . . . . . 83

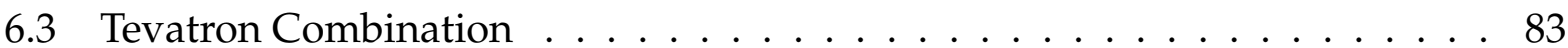


7 Search for New Resonances Decaying to a Top and a Bottom Quark 90

7.1 Definition of the Analysis Subsamples . . . . . . . . . . . . . . . . . . 91

7.2 Multivariate Discriminants . . . . . . . . . . . . . . . 91

7.3 Limit Extraction . . . . . . . . . . . . . . . . . . . . 95

8 Conclusions $\quad 100$

A Measurement of the Single Top Quark Production Cross Section and the CKM Matrix Element $\left|V_{t b}\right|$ at the Tevatron $\quad 101$

A.1 Measurement of Single Top Quark Production in the $\mathbb{E}_{T} \boldsymbol{b} \bar{b}$ Sample . . . . . 102

A.1.1 Multivariate Discriminants . . . . . . . . . . . . . . . . 102

A.1.2 Cross Section and $\left|V_{t b}\right|$ Limit Extraction . . . . . . . . . . . . . . . 103

A.2 CDF Combination . . . . . . . . . . . . . . . . 106

A.3 Tevatron Combination . . . . . . . . . . . . . . . . 107

B Validation Plots for the Single Top Analyses 113

B.1 Two-Jets EWK Sample . . . . . . . . . . . . . . . . . . . . . . . . . . 113

B.2 Three-Jets EWK Sample . . . . . . . . . . . . . . . . . . . . 116

B.3 Two-Jets QCD Sample . . . . . . . . . . . . . . . . . . . . . . . . 118

B.4 Three-Jets QCD Sample . . . . . . . . . . . . . . . . . . . . . . 120

C Validation Plots for the $W^{\prime}$ Boson Analysis 122

C.1 Two-Jets Lepton QCD Sample . . . . . . . . . . . . . . . . . . . . . . . . 122

C.2 Three-Jets Lepton QCD Sample . . . . . . . . . . . . . . . . . . . . . 125

C.3 Two-Jets No-Lepton QCD Sample . . . . . . . . . . . . . . . . . . . 128

C.4 Three-Jets No-Lepton QCD Sample . . . . . . . . . . . . . . . . . . . . 131 


\section{List of Figures}

1.1 Parton distribution functions at the scale $\mu=m_{t}$, relevant for top-quark production. From [14]. . . . . . . . . . . . . . . 7

1.2 Leading order Feynman diagram of the $t \bar{t}$ production via quark annihilation 8

1.3 Leading-order Feynman diagram of $t \bar{t}$ production via gluon fusion. . . . . . 8

1.4 Leading-order Feynman diagrams for electroweak single-top-quark production via the (a) s-channel, (b), (c), and (d) t-channel, and (e) associated $t W$ process. Diagrams with off-diagonal CKM matrix elements, such as $W t \bar{s}$ or $W t \bar{d}$ vertices, are suppressed due to the small values of their CKM matrix elements. . . . . . . . . . . . . . . . . 10

1.5 Comparison between expected limits for CDF, CMS, and ATLAS in a search for a $Z^{\prime}$ boson resonance decaying to $t \bar{t}$. The x-axis shows the value of $M_{Z^{\prime}}$ and the $y$-axis shows the $95 \%$ C.L. observed limit normalized to the theoretical cross section in a benchmark model. From [36] . . . . . . . . . . . . . 12

1.6 Feynman diagrams for $W^{\prime}$-boson $s$-channel production and subsequent leptonic decay of the $W$ boson originating from the $t$ quark. . . . . . . . . . . . 14

2.1 The Fermilab accelerator system and the Tevatron collider. . . . . . . . . . . 16

2.2 Tevatron integrated luminosity as a function of Run II week number (top) and peak luminosity as a function of calendar date (bottom). Empty regions correspond to Tevatron shut-down periods. . . . . . . . . . . . . . 19

2.3 Isometric view of the CDF II detector. . . . . . . . . . . . . . . . 20

2.4 (top) The CDF II tracker layout showing the different subdetector systems. (bottom) Schematic drawing of the impact parameter $d_{0}$. The sign of the impact parameter is defined as positive or negative with reference to the direction of the track momentum vector (in the $x, y$ quadrant in the drawing). The sign of the impact parameter is negative for tracks 2 and 3, positive for tracks 1 and 4.

2.5 The SVX silicon detector: on the left, a three-dimensional view of the detector shows the barrel structure along the beam axis; on the right, the transverse plane section shows in detail the layer sequence. The innermost layer L00 is supported by the beam pipe. . . . . . . . . . . . . . . . . . . 24 
2.6 (top) $60^{\circ}$ section of the COT end-plate. For each super-layer the total number of cells, the wire orientation (axial or stereo) and the average radius in $\mathrm{cm}$ are shown. The enlargement shows in detail the slot where the wire planes (sense and field) are installed. (bottom) Cross-section of three axial cells in super-layer 2; the arrow indicates the radial direction. . . . . . . . 25

2.7 (top) Artist's view of a central calorimeter wedge showing the pipes collecting light from the front electromagnetic towers; (bottom left) cut view of the plug; (bottom right) elevation view of the detector showing the components of the calorimeter system. . . . . . . . . . . . 28

2.8 Coverage of the muon detectors in the $\eta-\phi$ plane . . . . . . . . . . . . . . 30

2.9 Functional block diagram of the CDF II trigger and data acquisition systems. 33

3.1 Sketch of a jet shower with fragmentation and hadronization. . . . . . . . . 36

3.2 Candidate event of $W+$ jets production (run 166063, event 279746) with two secondary vertices tagged. The $\vec{E}_{T}$ direction, a muon track, a prompt track and tracks from the secondary vertices are shown . . . . . . . . . . . . . 40

3.3 Left: JETPROB distributions for jets matched to $b$ (full circles), $c$ (empty circles), and light (empty squares) quarks in MC simulated events. Right: JETPROB distributions for electron jets in inclusive electron data (full circles) and for generic QCD jets in Jet50 data (empty squares). From [62] . . . . 42

3.4 Distribution of the impact parameter significance for tracks in an inclusive jet sample with at least 5 good SVX hits, $p_{T}>5 \mathrm{GeV} / \mathrm{c}$, and $|\eta|<0.6$. From [62].

3.5 The HOBIT output distribution. The black histogram is for light-flavour jets and the red histogram is for $b$-jets. Both distributions are MC simulations normalized to unity. From [63] . . . . . . . . . . . . . . . . . . 4

3.6 The $b$-jet and light-jet efficiencies in MC before scale factor corrections as a function of $\eta$ and $E_{T}$. The black triangles are for the looser operating point and the coloured triangles are for the tighter operating point. From [63]. . . 45

3.7 The $b$-jet and light-jet efficiencies for the HOBIT tagger compared to the other CDF taggers. From [63] . . . . . . . . . . . . . . . . . . 45

4.1 Trigger efficiency for the $\mathbb{E}_{T}$-triggers as a function of $\mathbb{E}_{T}$ parametrized by a neural-network function in the (left) MUON and (right) JET-50 samples. The agreement of the MUON and JET-50 parameterizations in the samples where they are derived is simply a consistency check. From [72] . . . . . . . 53

4.2 The kinematic regions defined for events with two high- $E_{T}$ jets and $E_{T}$. . . 55

4.3 Schematic view of contribution from the SM processes to the selected data sample. The $V H$ production is not shown. . . . . . . . . . . . . . . 56

4.4 Illustration of the strategy for the background model. . . . . . . . . . . . . . 57

4.5 Representative Feynman diagrams for: (a) $W+H F,(b) Z+H F$, (c) $t \bar{t}$, (d) $s$ channel single top, (e) $t$-channel single top, and (f) $W W$ production. . . . . . 58 
4.6 The measured value of the $b$-tag scale factor for the HOBIT tagger as a function of the minimum HOBIT output value. Variations are shown assuming two values of the $t \bar{t}$ cross section. The straight lines are fits to the scale factors assuming the central value of the $t \bar{t}$ cross section $\left(\sigma_{t \bar{t}}=7.04\right.$ $\mathrm{pb}$ ), and $\sigma_{t \bar{t}}=6.336 \mathrm{pb}$, the more conservative case for the purpose of estimating uncertainties. The latter fit has been reflected through the central line to obtain a symmetric uncertainty band. From [63] . . . . . . . . . . . . 60

4.7 The measured value of the mistag scale factor for the HOBIT tagger as a function of the minimum HOBIT output value. Variations are shown assuming two values of the $t \bar{t}$ cross section. Parabolas are fit to the results assuming the central value of the $t \bar{t}$ cross section $\left(\sigma_{t \bar{t}}=7.04 \mathrm{pb}\right)$, and for $\sigma_{t \bar{t}}=6.336 \mathrm{pb}$. The latter has been reflected through the curve for the central value to obtain the depicted uncertainty band. From [63]. . . . . . . 61

4.8 Feynman diagrams for QCD multijet production. Usually no neutrino is present, except when it is produced in the decay of a hadron. . . . . . . . . . 62

6.1 The $b$-tag efficiency and the mistag rate as a function of jet $E_{T}$ in MC samples independent from the samples used in the training. The black triangles are for the HOBIT loose operating point and the colored triangles are for the HOBIT tight operating point. The $\mathrm{x}$-axis is extended compare to Figure 3.6 to show the behaviour of the $b$-tag efficiency and the mistag rate for high- $E_{T}$ jets. From $[101] \ldots \ldots \ldots \ldots$. . . . . . . . . . . . . . . . . .

6.2 Predicted and observed distributions in the signal region for some of the most separating kinematic variables used in the training of the $\mathrm{NN}_{\mathrm{QCD}}$, $\mathrm{NN}_{V \text { jets }}$ and $\mathrm{NN}_{t \bar{t}}$ discriminants in the two-jet subsample. . . . . . . . 76

6.3 Predicted and observed distributions in the signal region for some of the most separating kinematic variables used in the training of the $\mathrm{NN}_{\mathrm{QCD}}$, $\mathrm{NN}_{V \text { jets }}$ and $\mathrm{NN}_{t \bar{t}}$ discriminants in the three-jet subsample. . . . . . . . . 77

6.4 Predicted and observed final discriminant distributions in the signal region, for (a) 1T two-jet, (b) 1T three-jet, (c) TL two-jet, (d) TL three-jet, (e) TT two-jet and (f) TT three-jet event subsamples. . . . . . . . . . . . . . . . 79

6.5 The $\mathrm{NN}_{\mathrm{QCD}}$ output variable in the preselection region for the TT two-jet subsample. The strategy applied to derive the QCD multijet normalization is summarized. The subsample composed by the events that do not satisfy the $\mathrm{NN}_{\mathrm{QCD}}>0.55$ requirement is used to calculate a scale factor, as described in Section 4.3.3. The scale factor is applied to the events in the signal region, defined by the the $\mathrm{NN}_{\mathrm{QCD}}>0.55$ requirement. . . . . . . . 80

6.6 The posterior probability distribution for the CDF $\mathbb{E}_{T} b \bar{b}$ analysis. . . . . . . 82

6.7 Pseudo-experiment distributions for the background-only (red) and SMsignal-plus-background (blue) hypotheses from the CDF $\mathbb{E}_{T} b \bar{b}$ analysis. . . 82

6.8 The posterior probability distribution for the CDF combination. . . . . . . . 84 
6.9 Pseudo-experiment distributions for the background-only (red) and SMsignal-plus-background (blue) hypotheses from the CDF combination. . . . 84

6.10 Distribution of the discriminant histograms, summed for bins with similar signal-to-background ratio $(s / b)$. The expected sum of the backgrounds is shown by the unfilled histogram, and the total uncertainty of the background is represented by the gray shaded band. The expected s-channel signal contribution is shown by a filled blue histogram. . . . . . . . . . . . 86

6.11 The background-subtracted distribution of the discriminant histograms, summed for bins with similar signal-to-background ratio $(s / b)$. The background and s-channel signal (blue filled histogram) have been normalized to the most likely values returned from the likelihood fit and the uncertainty on the background uncertainty has also been constrained by the data (gray shaded band). . . . . . . . . . . . . . . . . 87

6.12 The posterior probability distribution for the combination of the CDF and D0 analysis channels compared with the theoretical prediction [22] . . . . . 87

6.13 Measured single-top-quark s-channel production cross sections from each of the individual analyses and various combinations of these analyses com-

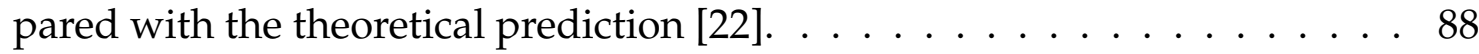

6.14 Log-likelihood ratios using an asymptotic approximation for the backgroundonly (solid green line) and SM-signal-plus-background (dashed blue) hypotheses from the combined measurement. . . . . . . . . . . . . . . 8

7.1 Predicted and observed distributions in the signal region for some of the most separating kinematic variables used in the training of the $\mathrm{NN}_{\mathrm{QCD}}$, $\mathrm{NN}_{V \text { jets }}$ and $\mathrm{NN}_{t \bar{t}}$ discriminants in the no-lepton two-jet subsample. . . . . 93

7.2 Predicted and observed distributions in the signal region for some of the most separating kinematic variables used in the training of the $\mathrm{NN}_{\mathrm{QCD}}$, $\mathrm{NN}_{V \text { jets }}$ and $\mathrm{NN}_{t \bar{t}}$ discriminants in the no-lepton three-jet subsample. . . . . 94

7.3 Predicted and observed distributions in the signal region for some of the most separating kinematic variables used in the training of the $\mathrm{NN}_{\mathrm{QCD}}$, $\mathrm{NN}_{V \text { jets }}$ and $\mathrm{NN}_{t \bar{t}}$ discriminants in the lepton two-jet subsample. . . . . . . 95

7.4 Predicted and observed distributions in the signal region for some of the most separating kinematic variables used in the training of the $\mathrm{NN}_{\mathrm{QCD}}$, $\mathrm{NN}_{V \text { jets }}$ and $\mathrm{NN}_{t \bar{t}}$ discriminants in the lepton three-jet subsample. . . . . . . 96

7.5 Predicted and observed final discriminant distributions in the signal region, for (a) 1T two-jet, (b) 1T three-jet, (c) TL two-jet, (d) TL three-jet, (e) TT two-jet and (f) TT three-jet event subsamples. Events from the no-lepton and lepton subsamples are merged. The distribution for a $W^{\prime}$ boson mass of $300 \mathrm{GeV} / c^{2}$ with SM coupling is superimposed. . . . . . . . . . . . . . 97 
7.6 Observed and expected limits on $\sigma\left(p \bar{p} \rightarrow W^{\prime}\right) \times \mathcal{B}\left(W^{\prime} \rightarrow t b\right)$, with $\pm 1 \sigma$ and $\pm 2 \sigma$ confidence intervals and theoretical predictions for a right-handed $W^{\prime}$ with SM-like couplings in cases where the leptonic decay mode $W^{\prime} \rightarrow$ $\ell v$ is allowed (solid line) or forbidden (dashed). The CDF limits are compared with observed limits from the latest $W^{\prime}$ boson searches from ATLAS [105], CMS [106] and D0 [104]. . . . . . . . . . . . . . . . . . . . 98

7.7 Expected $95 \%$ C.L. limits on the production cross section times branching ratio of a right-handed $W^{\prime}$ with SM coupling strength, normalized to the theoretical cross section times branching ratio as a function of $M_{W^{\prime}}$ in cases where the leptonic decay mode $W^{\prime} \rightarrow \ell v$ is forbidden. The CDF limits are compared with observed limits from the latest $W^{\prime}$ boson searches from ATLAS [105], CMS [106] and D0 [104]. . . . . . . . . . . . . . . . . . 99

A.1 Predicted and observed $N N_{\mathrm{sig}}{ }^{s+t}$ distributions in the signal region, for $1 \mathrm{~T}$ two-jet (a), 1T three-jet (b), TL two-jet (c), TL three-jet (d), TT two-jet (e) and TT three-jet (f) subsamples. . . . . . . . . . . . . . . . . . . . . 104

A.2 The posterior probability distribution for the for the $\mathbb{E}_{T} b \bar{b}$ analysis presented here. The orange region indicates the allowed values of $\left|V_{t b}\right|^{2}$ corresponding to the limit of $\left|V_{t b}\right|>0.63$ at $95 \%$ C.L. . . . . . . . . . 105

A.3 Two-dimensional posterior probability densities of the $s$ - and $t$-channel cross sections for the $\mathbb{E}_{T}^{t} b \bar{b}$ analysis presented here. . . . . . . . . . 106

A.4 The posterior probability distribution for the CDF combination of the $\mathbb{E}_{T} b \bar{b}$ and $\ell v b \bar{b}$ analysis results. The orange region indicates the allowed values of $\left|V_{t b}\right|^{2}$ corresponding to the limit of $\left|V_{t b}\right|>0.84$ at $95 \%$ C.L. . . . . . . 107

A.5 Two-dimensional posterior probability densities of the $s$ - and $t$-channel cross sections for the CDF combination of the $\mathbb{E}_{T} b \bar{b}$ and $\ell v b \bar{b}$ analysis results. . . . . . . . . . . . . . . . . . . . . . . . . . . 108

A.6 Distribution of the discriminant, summed over bins with similar ratios of (s-channel - $t$-channel) signals over background. Displayed are the data, $s$ - and $t$-channel production as predicted in the SM and the expected background. The total expected background (black solid line) is shown with uncertainty (gray shaded band). A nonlinear scale is used on the abscissa to better display the range of values. . . . . . . . . . . . . . . . . . 109

A.7 The 2D posterior probability with one, two, and three standard deviation probability contours for the combination of the CDF and D0 analysis channels compared with the theoretical prediction of the SM [22]. Several BSM predictions are also shown. . . . . . . . . . . . . . . . . . . 110

A.8 Measured single-top-quark production cross sections from the CDF and D0 Collaborations in different production channels and the Tevatron combinations of these analyses compared with the NLO+NNLL theoretical prediction [22]. 
A.9 The posterior probability distribution for the combination of CDF and D0 analysis channels. The region delimited by the solid blue line indicates the allowed values of $\left|V_{t b}\right|^{2}$ corresponding to the limit of $\left|V_{t b}\right|>0.92$ at $95 \%$ C.L.112 


\section{List of Tables}

1.1 Properties of the four fundamental forces of Nature. The strengths of interactions are shown relative to the strength of the electromagnetic force for two $u$ quarks separated by the specified distances. . . . . . . . . . . 2

1.2 The SM fundamental particles, with the gauge bosons in the bottom row. The values for the masses are from Ref. [8]. The Higgs boson, not included here, has properties consistent with those of the newly discovered boson at the $\mathrm{LHC} \ldots \ldots \ldots \ldots \ldots \ldots$

2.1 Accelerator parameters for Run II [43] . . . . . . . . . . . . . . . . 18

2.2 Summary of the main parameters of the CDF II calorimeter system. . . . . . 30

2.3 Design parameters of the muon detectors. See $[53,54] \ldots \ldots$

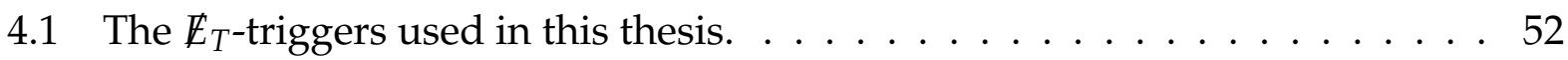

6.1 Numbers of predicted and observed two-jet events in the 1T, TL, and TT subsamples. The uncertainties on the predicted numbers of events are due to the theoretical-cross-section uncertainties and the uncertainties on signal and background modeling. Central values and uncertainties are obtained from the fit to the data which incorporates the theoretical constraints. . . . . 78

6.2 Numbers of predicted and observed three-jet events in the 1T, TL, and TT

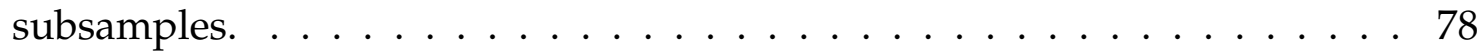

6.3 Systematic uncertainties associated with the CDF and D0 single top quark $s$-channel cross section measurements. The values shown for each category indicate the range of uncertainties applied to the predicted normalizations for signal and background contributions over the full set of analysis samples from each experiment. The black dots indicate which categories contribute uncertainties on the shape of the final multivariate discriminant output variable. It is also noted if categories are treated as fully correlated between the two experiments. . . . . . . . . . . . . . 8 
7.1 Numbers of predicted and observed two-jet events with and without identified leptons, in the 1T, TL, and TT subsamples. The uncertainties on the predicted numbers of events are due to the theoretical and experimental uncertainties on signal and background modeling. Expected number of events for a right-handed $W^{\prime}$ boson with SM-like couplings and 300 $\mathrm{GeV} / c^{2}$ mass are shown. . . . . . . . . . . . . . . . . . 92

7.2 Numbers of predicted and observed three-jet events with and without identified leptons, in the 1T, TL, and TT subsamples. The uncertainties on the predicted numbers of events are due to the theoretical and experimental uncertainties on signal and background modeling. Expected number of events for a right-handed $W^{\prime}$ boson with SM-like couplings and 300 $\mathrm{GeV} / c^{2}$ mass are shown. . . . . . . . . . . . . . . . . . . 92

A.1 Numbers of predicted and observed events in the two-jet signal region in the subsamples with exactly one tightly tagged jet (1T), one tightly and one loosely tagged jet (TL), and two tightly tagged jets (TT). The uncertainties in the predicted numbers of events are due to the theoretical cross section uncertainties and to the uncertainties on signal and background modeling. Both the uncertainties and the central values are those determined by the fit to the data with theory constraints. . . . . . . . . . . . . . 103

A.2 Numbers of predicted and observed three-jet events in the 1T, TL, and TT subsamples. . . . . . . . . . . . . . . . . . . . . 105 


\section{Chapter 1}

\section{Theoretical Background}

Our understanding of Nature is based on the existence of four fundamental forces: the gravitational, electromagnetic, weak and strong forces. Significant effort in theoretical physics is directed towards the unification of all interactions under a complete theory. Currently, the electromagnetic, weak and strong forces are included in a larger framework called the Standard Model (SM) of the fundamental interactions. Since it does not include gravitation, the search for a universal model is still ongoing.

The SM is a theory that describes most of the elementary particle physics processes. It successfully predicted the existence of new particles, like the $W$ and $Z$ bosons and the top quark, that were later experimentally confirmed. As of July 2012, the missing piece was the evidence for the existence of the Higgs boson. The discovery of a new particle of mass about $125 \mathrm{GeV} / \mathrm{c}^{2}$, showing many of the features expected for the SM Higgs boson, was announced at CERN in 2012 [1, 2]. Although not yet conclusive, the ongoing studies to ascertain its nature seem to confirm that this particle is indeed the SM Higgs boson [3, 4].

A vast number of different models, usually referred to as physics beyond the Standard Model (BSM), have been proposed to replace or extend the SM. One of the goals of the Tevatron and LHC physics programs has been testing BSM models, but currently they have found no clear evidence of discrepancies with SM expectations. The search for new physics will be the main focus of the LHC Run II physics program.

\subsection{The Standard Model}

The SM is a theory for particle physics based on the gauge group $S U(3) \times S U(2)_{L} \times$ $U(1)_{Y}$. The strong force, which is described by quantum chromodynamics (QCD), is represented by $S U(3)$, where 3 indicates the number of rows (and columns) in the unitary matrices forming the group. It can be thought of as the number of charges, or colours, 
possessed by elements in the fundamental representation of the group (quarks). In 1961, Glashow [5] proposed an unified gauge theory for the electromagnetic and the weak interactions based on $S U(2)_{L} \times U(1)_{Y}$ group symmetry. The subscript $L$ indicates that the weak interaction only acts on the left-handed fermions. $Y$ is the generator of $U(1)_{Y}$, and is called the weak hypercharge.

\subsubsection{Fundamental Forces and Particles}

There are four known fundamental forces of Nature: the gravitational force, the electromagnetic force, the weak force, and the strong force. All of these forces, except gravity, are described by the SM. The properties of the fundamental forces, or interactions, are summarized in Table 1.1.

Table 1.1 - Properties of the four fundamental forces of Nature. The strengths of interactions are shown relative to the strength of the electromagnetic force for two $u$ quarks separated by the specified distances.

\begin{tabular}{|c|c|c|c|c|}
\hline Force & Gravitational interaction & Weak interaction & Electromagnetic interaction & Strong interaction \\
\hline Acts on: & Mass - Energy & Flavour & Electric charge & Colour charge \\
\hline Particles experiencing & All & Quarks, leptons, and $W / Z$ bosons & Electrically charged & Quarks and gluons \\
\hline Particles mediating & Graviton (not yet observed) & $Z^{0}, W^{ \pm}$ & $\gamma$ & Gluons \\
\hline Strength at $10^{-18} \mathrm{~m}$ & $10^{-41}$ & 0.8 & 1 & 25 \\
\hline Strength at $3 \times 10^{-17} \mathrm{~m}$ & $10^{-41}$ & $10^{-4}$ & 1 & 60 \\
\hline
\end{tabular}

Some features of the forces are as follows. The electromagnetic force acts between electrically charged particles and is responsible for atomic bound states. The strong force holds quarks together to form hadrons by acting on the colour charge. The strong force also binds protons and neutrons to form nuclei. It is the strongest force at the nuclear scale. The weak force was discovered in beta decay $\left(n \rightarrow p+e^{-}+\bar{v}_{e}\right)$, in which a continuous energy spectrum of the electron was observed. The strength of the weak force is comparable to the electromagnetic force at $10^{-18} \mathrm{~m}$, while its strength is $10^{-4}$ that of the electromagnetic force at $3 \times 10^{-17} \mathrm{~m}$.

According to the SM [6], all fundamental particles are either bosons or fermions.

Particles associated with the matter fields-leptons and quarks-are fermions. They have half-integer spin and are divided into three families, or six flavours each for quarks and leptons. The quarks have electric charge, colour charge, baryon number and flavour quantum number, and they are the only fundamental particles in the SM to experience all four fundamental forces. There are two kinds of leptons: charged leptons (like the electron) and neutral leptons (the neutrinos).

Gauge bosons are the force carriers for the interactions of the matter fields. Fundamental particles, whose interactions are described by a gauge theory, interact with each other by the exchange of gauge bosons. Gauge bosons have spin equal to one. There are three kinds of gauge bosons in the SM: photons, which are massless and mediate the electro- 
magnetic interaction; $W^{ \pm}$and $Z$ bosons, which have masses on the order of $100 \mathrm{GeV} / c^{2}$ and carry the weak force; and gluons, predicted to be massless, which carry the strong force. The existence of a Higgs boson is a consequence of the Higgs mechanism [7], included in the SM as the mechanism that gives mass to fundamental particles.

Overall, the SM includes six types of quarks and six types of leptons, and their corresponding anti-particles; the photon and $W^{ \pm}$and $Z$ bosons; eight coloured gluons, and the Higgs boson. A summary of the properties of these particles is shown in Table 1.2.

Table 1.2 - The SM fundamental particles, with the gauge bosons in the bottom row. The values for the masses are from Ref. [8]. The Higgs boson, not included here, has properties consistent with those of the newly discovered boson at the LHC.

\begin{tabular}{cccccc}
\hline \hline & Name & Symbol & Spin $(\hbar)$ & Charge $(e)$ & Mass $\left(\mathrm{MeV} / c^{2}\right)$ \\
\hline \multirow{5}{*}{ Leptons } & $e$ neutrino & $v_{e}$ & $1 / 2$ & 0 & $<2 \cdot 10^{-6}$ \\
& electron & $e$ & $1 / 2$ & -1 & $0.510998910 \pm 0.000000013$ \\
\cline { 2 - 6 } & $\mu$ neutrino & $v_{\mu}$ & $1 / 2$ & 0 & $<0.19$ \\
& muon & $\mu$ & $1 / 2$ & -1 & $105.6583668 \pm 0.0000038$ \\
\cline { 2 - 6 } & $\tau$ neutrino & $v_{\tau}$ & $1 / 2$ & 0 & $<18.2$ \\
& tau & $\tau$ & $1 / 2$ & -1 & $1776.82 \pm 0.16$ \\
\hline \hline \multirow{5}{*}{ Quarks } & up & $u$ & $1 / 2$ & $2 / 3$ & $2.49 \pm_{0.79}^{0.81}$ \\
& down & $d$ & $1 / 2$ & $-1 / 3$ & $5.5 \pm_{0.95}^{0.75}$ \\
\cline { 2 - 6 } & charm & $c$ & $1 / 2$ & $2 / 3$ & $\left(1.27 \pm_{0.09}^{0.07}\right) \cdot 10^{3}$ \\
& strange & $s$ & $1 / 2$ & $-1 / 3$ & $101 \pm 29$ \\
\cline { 2 - 6 } Gauge Bosons & beauty & $b$ & $1 / 2$ & $2 / 3$ & $\left(4.19 \pm_{0.06}^{0.18}\right) \cdot 10^{3}$ \\
& top & $t$ & $1 / 2$ & $-1 / 3$ & $\left(172.0 \pm \pm_{1.3}^{0.9}\right) \cdot 10^{3}$ \\
\hline \hline & photon & $\gamma$ & 1 & 0 & 0 \\
& $W$ boson & $W^{ \pm}$ & 1 & \pm 1 & $(80.399 \pm 0.023) \cdot 10^{3}$ \\
& $Z$ boson & $Z^{0}$ & 1 & 0 & $(91.1876 \pm 0.0021) \cdot 10^{3}$ \\
& gluon & $g$ & 1 & 0 & 0 \\
\hline \hline
\end{tabular}

\subsubsection{The Electroweak Model and the Higgs Mechanism}

In quantum electrodynamics the electromagnetic amplitudes are calculated using an electromagnetic current $j_{\mu}^{e m}$ that is coupled to an electromagnetic field $A^{\mu}$ :

$$
-i e j_{\mu}^{e m} A^{\mu}=-i e\left(\bar{\Psi} \gamma_{\mu} Q \Psi\right) A^{\mu} \quad U(1)_{E M}
$$

where $Q$ is the charge of the fermion field $\Psi$. Similarly, the weak amplitudes are calculated using an isotriplet of weak currents $J_{\mu}$ :

$$
J_{\mu}=\frac{1}{2} \bar{\Psi}_{i}^{L} \gamma_{\mu} \tau \Psi_{i}^{L}
$$


where $\tau$ are the Pauli spin matrices and $\Psi_{i}^{L}$ is the weak isospin doublet, defined by the left-handed lepton fields $v_{i}, l_{i}$ and the left-handed quark fields $u_{i}, d_{i}^{\prime}$ :

$$
\Psi_{i}^{L}=\left(\begin{array}{c}
v_{i} \\
l_{i}
\end{array}\right)_{L} \text { and }\left(\begin{array}{c}
u_{i} \\
d_{i}^{\prime}
\end{array}\right)_{L},
$$

where the subscript $i$ runs over the different fermion families, $d_{i}^{\prime}=\sum_{j} V_{i j} d_{j}{ }^{1}$, and $V$ is the Cabbibo-Kobayashi-Maskawa mixing matrix [9].

The weak hypercharge can be defined from the electric charge $Q$ and the weak isospin charges $I_{3}^{W}$ :

$$
\frac{Y}{2}=Q / e-I_{3}^{W}
$$

so that left-handed neutral leptons $v_{i}$ have $I_{3}^{W}=\frac{1}{2}, Y=-1$ and the left-handed charged leptons have $I_{3}^{W}=-\frac{1}{2}, Y=-1$.

Electromagnetic and weak forces are unified in a gauge theory based on a $S U(2)_{L} \times U(1)_{Y}$ group symmetry, where the weak current $J_{\mu}$ is coupled to three vector gauge fields $W^{\mu}$ for weak interaction:

$$
-i g J_{\mu} W^{\mu}=-i g \bar{\Psi}_{i}^{L} \gamma_{\mu} \tau W^{\mu} \Psi_{i}^{L} \quad S U(2)_{L}
$$

and the weak hypercharge current $j_{\mu}^{Y}$ is coupled to a vector gauge field $B^{\mu}$ for electromagnetic interaction:

$$
-i \frac{g^{\prime}}{2} j_{\mu}^{Y} B^{\mu}=-i g^{\prime}\left(\bar{\Psi} \gamma_{\mu} \frac{Y}{2} \Psi\right) B^{\mu} \quad U(1)_{Y} .
$$

The operators $\tau$ and $Y$ are the generators of the $S U(2)_{L}$ and $U(1)_{Y}$ groups of gauge transformations, respectively, and $g$ and $g^{\prime}$ are the coupling constants.

The photon and massive bosons can be written as linear combinations of $W_{\mu}^{i}, B_{\mu}$ and $\theta_{W}$, where $\theta_{W}$ is the Weinberg angle or weak mixing angle:

$$
\begin{array}{lrl}
\text { photon } & A^{\mu} & =B^{\mu} \cos \theta_{W}+W_{3}^{\mu} \sin \theta_{W} \\
\text { neutral weak boson } & Z^{\mu} & =-B^{\mu} \sin \theta_{W}+W_{3}^{\mu} \cos \theta_{W}, \\
\text { charged weak boson } & W_{ \pm}^{\mu} & =\left(W_{1}^{\mu} \mp i W_{2}^{\mu}\right) / \sqrt{2}
\end{array}
$$

where

$$
\theta_{W}=\tan ^{-1} \frac{g^{\prime}}{g}
$$

The invariant Lagrangian energy density is written as:

$$
\mathcal{L}=\frac{g}{\sqrt{2}}\left(J_{\mu}^{-} W_{\mu}^{+}+J_{\mu}^{+} W_{\mu}^{-}\right)+\frac{g}{\cos \theta_{W}}\left(J_{\mu}^{(3)}-\sin ^{2} \theta_{W} J_{\mu}^{e m} Z_{\mu}\right)+g \sin \theta_{W} J_{\mu}^{e m} A_{\mu} .
$$

\footnotetext{
${ }^{1}$ With $d_{i}^{\prime}$ is indicated the weak interaction state, while with $d$ is indicated the mass eigenstates
} 
The first and second terms represent the weak charged current and the neutral current, respectively. The third term represents the electromagnetic neutral current.

In order to retain gauge invariance, the fields $W_{\mu}^{i}$ and $B_{\mu}$ should be massless. The Higgs mechanism is thus introduced to generate the particle masses, keeping gauge invariance.

The four Higgs fields are arranged in an isospin doublet with weak hypercharge $Y=1$ :

$$
\phi=\left(\begin{array}{c}
\phi^{+} \\
\phi^{0}
\end{array}\right) \text { with } \begin{aligned}
& \phi^{+}=\left(\phi_{1}+i \phi_{2}\right) / \sqrt{2} \\
& \phi^{0}=\left(\phi_{3}+i \phi_{4}\right) / \sqrt{2}
\end{aligned} .
$$

The Higgs doublet generates $W^{ \pm}$and $Z$ masses. The Yukawa couplings between the Higgs doublet and the fermion fields is responsible for the masses of the leptons and quarks.

\subsubsection{Quantum Chromodynamics}

Quantum Chromodynamics is the theory of the strong interactions between quarks and gluons. A single colour charge is assigned to quarks while gluons, the mediators of the strong force, carry a colour and an anti-colour charge. In $S U(3)$, three different colour charges can be assigned, leading to a total of nine states for gluons, collected into a colour octet and colour singlet (which is colourless). Quarks only exist in bound states with integer electromagnetic charge: quark-antiquark pairs (mesons), or three quarks or antiquarks (baryons). Mesons and baryons are subject to strong interactions and are called hadrons. The main feature of the strong force is that the interaction becomes stronger as the distance increases. In perturbative QCD, the coupling constant $\alpha_{S}$ is a function of the momentum transferred in the interaction, $q$, and is approximately given by

$$
\alpha_{S}\left(q^{2}\right)=\frac{12 \pi}{\left(33-2 n_{f}\right) \log \left(q^{2} / \Lambda_{Q C D}^{2}\right)},
$$

where $\Lambda_{Q C D} \approx 0.1 \mathrm{GeV}$ and $n_{f}$ is the number of quark flavours whose mass is greater than the $q^{2}$ of the interaction [10].

At very short distances, corresponding to very large $q^{2}$, the interaction becomes very weak. This effect is known as asymptotic freedom. It allows the use of perturbative expansions to calculate high- $q^{2}$ cross sections, which are relevant for the collisions at the Tevatron.

Production of quarks and gluons in a high energy physics experiment results in a process called hadronization or showering: when a parton is produced in an interaction, the potential tries to keep it bound until the energy is sufficient to create $q \bar{q}$ pairs. The new partons are approximately collinear with the original parton and combine into mesons or baryons such that a jet of colourless particles is observed. 
During the fragmentation, particle energies become successively smaller and perturbative QCD is no longer applicable. This makes most fragmentation processes non-perturbative and not analytically predictable. In order to describe jet properties [13], phenomenological models are usually applied and included in the Monte Carlo description of the jet kinematics.

\subsection{The Top Quark}

The top quark was discovered in 1995 by the CDF and D0 Collaborations at the Tevatron accelerator at Fermilab [11]. The top quark completes the third quark generation and interacts with other particles through the electroweak and strong forces. The most recent world combination of measurements performed at the Tevatron and at the LHC yields a top-quark mass of $m_{t}=173.34 \pm 0.27$ (stat) \pm 0.71 (syst) $\mathrm{GeV} / c^{2}$ [12], indicating that the top quark is the heaviest known fundamental particle. Its mass is about 35 times larger than its weak partner, the bottom quark. Measurements of the top quark mass and of the $W$ boson mass can be used to set indirect constrains on the Higgs boson mass. Comparing this with the direct measurement of the Higgs boson mass, deviations would indicate hints of new physics. Given its mass, the predicted top-quark lifetime is $\tau \approx 5 \times 10^{-25} \mathrm{~s}$, one order of magnitude smaller than the time scale for hadronization. Therefore, the top quark does not form bound states but decays through the weak interaction. In the SM the top quark decays nearly $100 \%$ of the time into a $b$-quark and a $W$ boson, while the decays to light-flavour quarks (down or strange) are suppressed because of the very small values of the $\left|V_{t d}\right|$ and $\left|V_{t s}\right|$ elements of the CKM quark mixing matrix.

\subsubsection{Top-Pair Production Through the Strong Interaction}

In the SM, interaction cross sections between quarks and gluons can be calculated. However, at the Tevatron the collisions are between protons and antiprotons, bound states of two $u$ quarks and one $d$ quark, and of their antiparticles, respectively. These quarks are called valence quarks and they interact through virtual gluons that can split into quarkantiquark pairs, called sea quarks. The total momentum $P$ of the proton (or antiproton) is distributed among the constituent partons, each one carrying a fraction $x$ of the parent hadron momentum. The momentum carried by parton $i$ is given by $P_{i}=x_{i} \cdot P$ and its distribution is described by parton distribution functions (PDFs) $f_{i}\left(x_{i}, \mu^{2}\right)$, corresponding to the probability to find the given parton inside the hadron with momentum fraction $x_{i}$, when probed at an energy scale $\mu^{2}$. In top-quark production the typical energy scale of the interaction is usually set to the top mass $\mu=m_{t}$. Since the Tevatron is a $p \bar{p}$ collider with symmetric beam energy, the square of the centre of mass energy of two interacting 
partons is given by

$$
\hat{s}=\left(x_{1} P_{p}+x_{2} P_{\bar{P}}\right)^{2}=4 x_{1} x_{2} s,
$$

where $s$ is the square of the centre of mass energy of the colliding protons and antiprotons ( $\sqrt{s}=1.96 \mathrm{TeV}$ for Run II of the Tevatron). The minimum square centre of mass energy of the interacting partons required to produce a $t \bar{t}$ pair is $\hat{s}_{\min }=4 m_{t}^{2}$. This leads to a $\hat{s}_{\text {min }}$ value of about $3.2 \%$ of $s$, so

$$
x_{1} x_{2} \geq \frac{\hat{s}_{\min }}{s} \approx 0.032
$$

At these values the partons responsible for the production of the top-quark pairs are mostly valence quarks, as can be seen in Figure 1.1. The figure shows that $x f(x)$ is largest for gluons at $\mathrm{x}<0.18$.

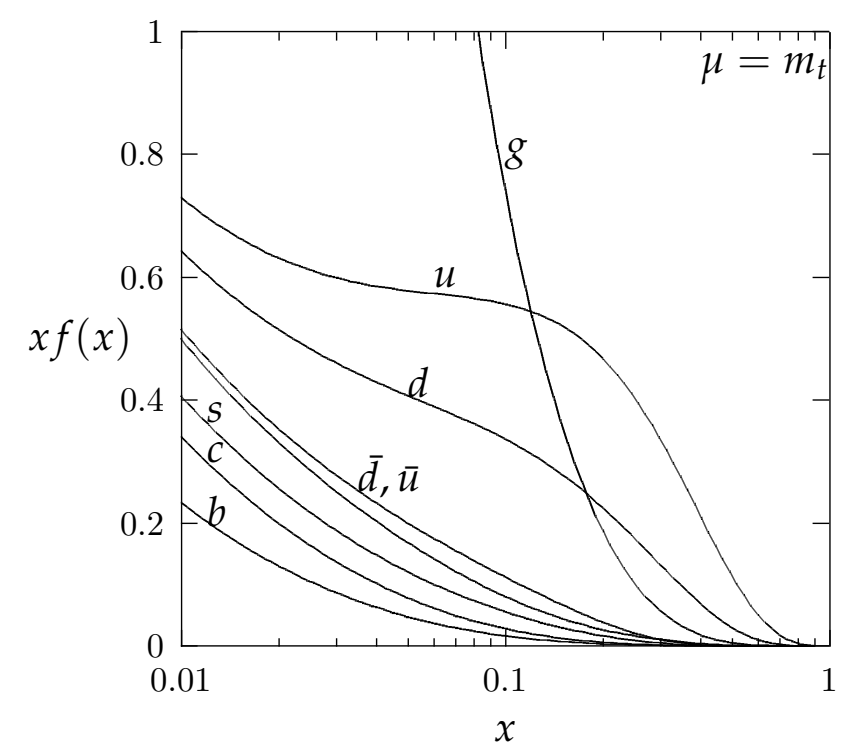

Figure 1.1 - Parton distribution functions at the scale $\mu=m_{t}$, relevant for top-quark production. From [14].

Calculations show that at the Tevatron roughly $85 \%$ of the production cross section is due to quark-antiquark annihilation, while the remaining fraction is due to gluon interactions [15]. The production rate is computed by integrating the production cross sections of all involved partons over their structure functions:

$$
\sigma_{p \bar{p} \rightarrow t \bar{t}}\left(\sqrt{s}, m_{t}\right)=\sum_{i, j=q, \bar{q}, g} \int \hat{\sigma}_{i j \rightarrow t \bar{t}}\left(\sqrt{\hat{s}}, m_{t}\right) f_{i}\left(x_{i}, m_{t}\right) f_{j}\left(x_{j}, m_{t}\right) d x_{i} d x_{j} .
$$

The parton-parton cross section can be calculated as a perturbation series in the strong coupling constant $\alpha_{s}\left(m_{t}^{2}\right)$. The leading-order (LO) Feynman diagrams of $t \bar{t}$ production are shown in Figures 1.2 and 1.3. At next-to-leading order (NLO), contributions due to initialstate and final-state radiation, gluon splitting and bremsstrahlung are added to the leading order calculation. The current next-to-next-to-leading order (NNLO) approximated topquark pair-production cross section at the Tevatron is $7.24_{-0.27}^{+0.23} \mathrm{pb}$ [16] for a top quark with mass $m_{t}=172.5 \mathrm{GeV} / c^{2}$. 


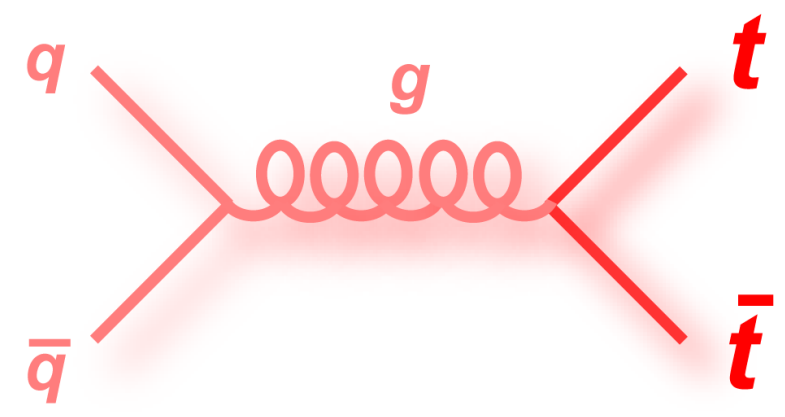

Figure 1.2 - Leading order Feynman diagram of the $t \bar{t}$ production via quark annihilation

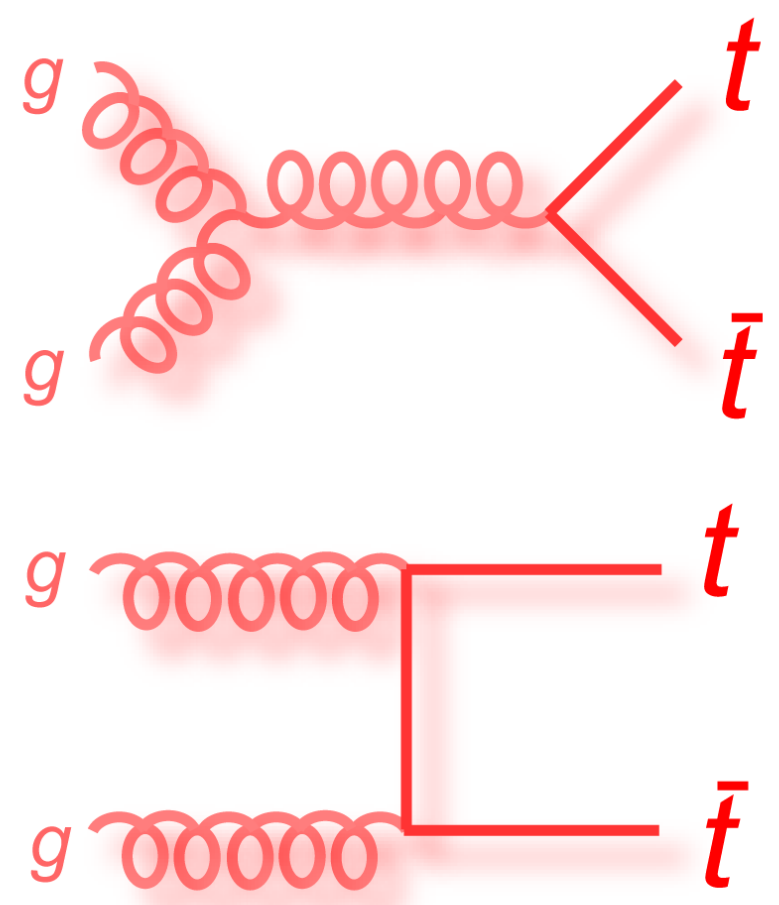

Figure 1.3 - Leading-order Feynman diagram of $t \bar{t}$ production via gluon fusion.

\subsubsection{Electroweak Single Top Quark Production}

Top quarks can also be produced singly without their antiparticle partners via the electroweak interaction. Three channels of single-top-quark production are possible: $s$-channel, $t$-channel, and associated $t W$ production (all indicated in Figure 1.4). The letters $s$ and $t$ refer to the partonic kinematic invariants (Mandelstam variables) for the process $p_{1}+p_{2} \rightarrow$ $p_{3}+p_{4}$, where the partons are represented by their four-momenta $p_{i}$, with $s=\left(p_{1}+p_{2}\right)^{2}$, and $t=\left(p_{1}-p_{3}\right)^{2}$. Charge-conjugate processes (the production of antitop quarks) are expected to have the same diagrams and rates at the Tevatron. Throughout this dissertation, the charge-conjugate processes are included, unless the contrary is explicitly stated. 
The s-channel corresponds to the process $q^{\prime} \bar{q} \rightarrow t \bar{b}$ shown in Figure 1.4(a) [17, 18, 19, 20, 21]: an initial-state quark-antiquark pair annihilates into an off-shell virtual, timelike $\left[q_{W}^{2}=s \geq\left(m_{t}+m_{b}\right)^{2}\right] W$ boson, which then decays into a top quark and a $b$-quark. Except for collinear production, the final state $b$-quark recoils against the top quark with large transverse momentum. Since the $W$ boson does not interact through the strong force, the initial state of the s-channel must be a colour singlet, which limits the number of initial colour combinations and therefore the production rate. For a top quark mass of $m_{t}=172.5 \mathrm{GeV} / c^{2}$, the predicted NNLO cross section is $1.05 \pm 0.06 \mathrm{pb}^{2}$ at $\sqrt{s}=1.96 \mathrm{TeV}$ at the Tevatron [22], while it is $4.56_{-0.18}^{+0.19} \mathrm{pb}$ at $\sqrt{s}=7 \mathrm{TeV}$ at the LHC [23]. At the LHC the $s$-channel cross section increases slowly with centre-of-mass energy since the initial antiquark must be provided from the sea. Together with a higher background contamination, at the LHC the s-channel cross section is difficult to measure so the Tevatron provides a higher purity sample with which to perform this measurement. Since the $t$-channel has a higher fraction of initial-state gluons and the uncertainty on gluon distribution functions is larger, the $s$-channel cross section can be calculated more reliably than the $t$-channel cross section because the quark and antiquark distribution functions at the energy scales of the Tevatron and LHC are well known [18].

The $t$-channel corresponds to the process $q^{\prime} b \rightarrow t q$ or $q^{\prime} g \rightarrow t q \bar{b}[21,24,25,26,27]$, where a spacelike $\left(q_{W}^{2}=t<0\right) W$ boson, with imaginary (virtual) mass, is exchanged. In the $q^{\prime} b \rightarrow t q$ subprocess [Figure 1.4(b)], a $b$-quark from the sea couples with the virtual $W$ boson, producing a top quark. In the $q^{\prime} g \rightarrow t q \bar{b}$ subprocess [Figure 1.4(c)], one of the $b$ quarks originates from gluon splitting, couples with the virtual $W$ boson and produces a top quark, whereas the other $b$-quark tends to continue along the original direction of the incident gluon, with a small transverse momentum. Since the first process is suppressed by the PDFs of $b$-quarks in the sea, the second process gives the largest contribution to the $t$-channel cross section. For $m_{t}=172.5 \mathrm{GeV}$, the predicted cross section for all $t$-channel contributions is $2.10 \pm 0.12 \mathrm{pb}$ at the Tevatron $(\sqrt{\mathrm{s}}=1.96 \mathrm{TeV})$ [22], and $64.6_{-0.7-1.7}^{+2.1+1.5} \mathrm{pb}^{3}$ at the LHC $(\sqrt{s}=7 \mathrm{TeV})$ [28]. The $t$-channel cross section is the largest of the three modes of single top-quark production at both the Tevatron and LHC, and it is far larger at the LHC. This is mainly due to the gluon colour-octet nature in the initial state, so the number of colour combinations in the $t$-channel is greater than that in the $s$-channel, leading to larger $t$-channel production cross section.

The associated $t$-quark and $W$-boson production, or $t W$, corresponds to the process $b g \rightarrow$ $t W$ of Figure 1.4(e) $[29,30]$, where an on-shell $W$ boson $\left(q_{W}^{2}=m_{W}^{2}\right)$ is produced together with a top quark. The predicted cross section for this process is $0.28 \pm 0.06 \mathrm{pb}$ [29] at the

\footnotetext{
${ }^{2}$ Unless explicitly mentioned, all the uncertainties on the cross section calculations shown in this paragraph are the total theorical uncertainties, that include scale, PDF, $\alpha_{s}$ uncertainties.

${ }^{3}$ The first error set denotes the sum of the renomalisation and factorisation scale uncertainties, the second the $\mathrm{PDF}+{ }_{s}$ error.
} 


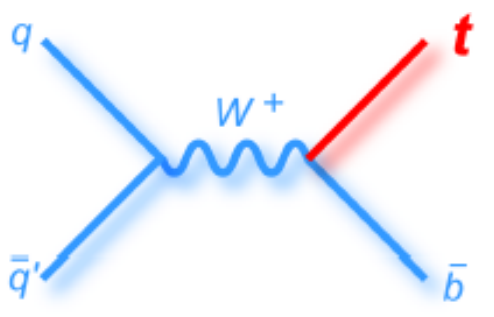

(a)

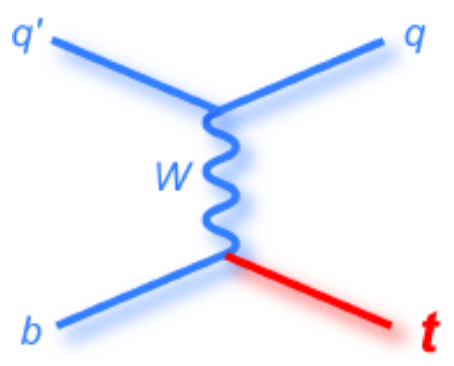

(b)

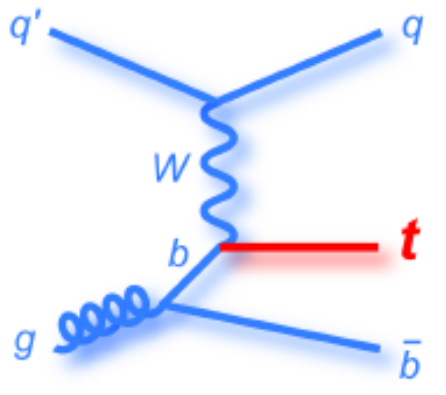

(c)

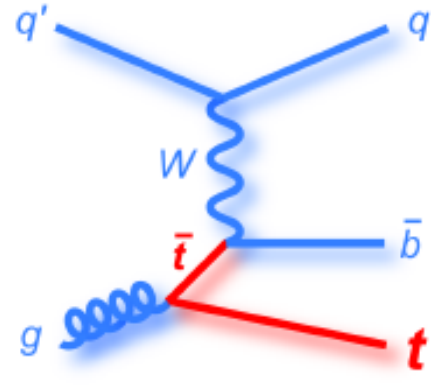

(d)
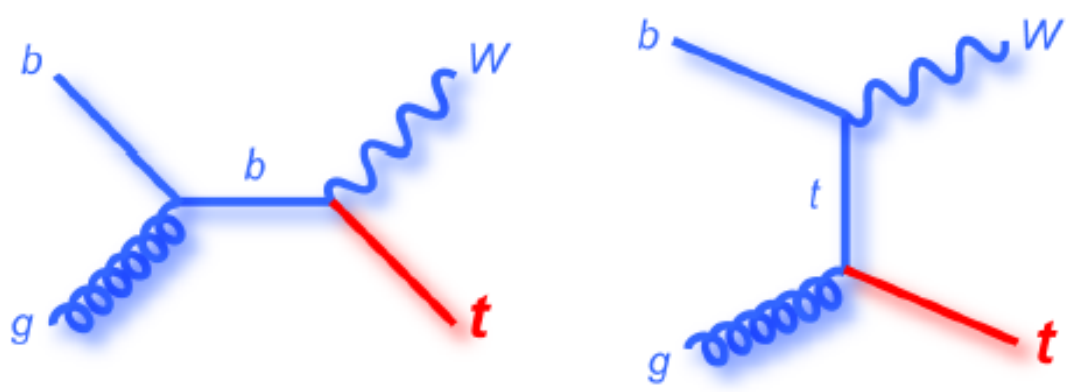

(e)

Figure 1.4 - Leading-order Feynman diagrams for electroweak single-top-quark production via the (a) s-channel, (b), (c), and (d) $t$-channel, and (e) associated $t W$ process. Diagrams with off-diagonal CKM matrix elements, such as $W t \bar{s}$ or $W t \bar{d}$ vertices, are suppressed due to the small values of their CKM matrix elements. 
Tevatron, where it can be neglected ${ }^{4}$. The $t W$ cross section is $15.6 \pm 0.4_{-1.2}^{+1.0} \mathrm{pb}^{5}$ at $\sqrt{s}=7$ $\mathrm{TeV}$ at the LHC [30].

\subsubsection{Top-Quark Decay}

Assuming the existence of three quark generations, the unitarity of the CKM matrix constrains the $\left|V_{t b}\right|$ element to be very close to unity [31]. Consequently, the top quark decays into a $W$ boson and a $b$-quark nearly $100 \%$ of the time. The $W$ boson can decay into one of the three charged-lepton-neutrino $\left(\ell v_{\ell}\right)$ pairs or into three coloured doublets of up and down $(u \bar{d})$ quarks or of charm and strange $(c \bar{s})$ quarks. The final state of top-quark decay is therefore either $\ell v b$ or $q \bar{q}^{\prime} b$, where $q$ and $\bar{q}^{\prime}$ denote quarks in the first and second generations ("light-flavoured quarks"6).

\subsection{Searching for a New Particle}

Although the SM predicts phenomena that have been experimentally proven, it shows several limitations. Neutrinos are predicted as massless while several measurement showed the presence of non-zero mass $[32,33]$. The SM does not include any prediction for dark matter particles with the properties deduced from the cosmological evidence, as well as any explanation for dark energy. Also, it does not incorporate the full theory of gravitation as described by general relativity. Since the SM can not be considered as a complete theory of fundamental interactions, a goal of many particle physics experiments is to search for new phenomena or new particles. Limitations in an experiment's sensitivity to new particles arise from either the production or the detection of the particle, or both. Particles can be difficult to produce if they have a small coupling to known SM particles or if their mass is near the upper limit of the accelerator's capabilities. Detection is difficult for particles with small couplings, but their presence can still be inferred from the energy and momentum they carry away from an interaction, as is done for neutrino identification. Detection is also difficult if particles are massive and quickly decay into ordinary quarks and leptons. For particles with masses well above that of the top quark, decay to a large number of particles is energetically possible, complicating the types of signals for which one must search. The challenge is to identify a few unusual events in a sea of ordinary interactions.

\footnotetext{
${ }^{4}$ Even though the $t W$ cross section is almost one fourth of the s-channel cross section, $t W$ production is neglected since the kinematic properties of this process can not be distinguished from kinematic properties of the $t \bar{t}$ production, resulting in an enhancement of just $4 \%$ to the background composed by top-antitop events.

${ }^{5}$ The first error set denotes the sum of the renomalisation and factorisation scale uncertainties, the second the $\mathrm{PDF}+{ }_{s}$ error.

${ }^{6}$ Since the mass of the charm quark is closer to the mass of the light-flavoured quarks $u, d, s$, than to the $b$-quark mass, we categorize it as a light-flavoured quark.
} 
Much of the evolution of particle physics has been based on the discovery of new patterns and symmetries. Most theoretical predictions for modifying or extending the SM are based on plausible new symmetries which include additional particles. To narrow the focus for new particles, some general assumptions are made in this thesis. We consider the generic case that some SM particles are mirrored at higher mass scales, remaining undiscovered; this is analogous to the second and third generation particles that are identical to the first generation particles but with larger masses.

With the CDF data there is still an opportunity to sharpen the search for massive new electroweak bosons, denoted $W^{\prime}$ and $Z^{\prime}$ bosons. The CDF Collaboration has recently completed a search for $Z^{\prime} \rightarrow t \bar{t}$ [34] with the full dataset, which is so far the most sensitive search for $t \bar{t}$ resonances decaying to a top-antitop pair below $730 \mathrm{GeV}$. The most recent analyses from Tevatron/LHC experiments have already excluded a benchmark leptophobic $Z^{\prime}$ model for $Z$ ' boson masses below $1 \mathrm{TeV}$. With the full Run II dataset CDF is sensitive to models with a lower cross section and/or lower branching ratios. The cross section for $\mathrm{SM} t \bar{t}$ production at the LHC is almost twenty times higher than at the Tevatron; as a result, LHC analyses are affected by a larger background of $t \bar{t}$ production. Although the cross section for a narrow $Z^{\prime}$ boson with mass below $730 \mathrm{GeV}$ is five times higher at the LHC [35], CDF has higher sensitivity to such a particle. Figure 1.5 shows that CDF is sensitive to possible narrow resonances with a cross section times branching ratio to $t \bar{t}$ ten times lower than benchmark $Z^{\prime}$ boson model in the low mass region.

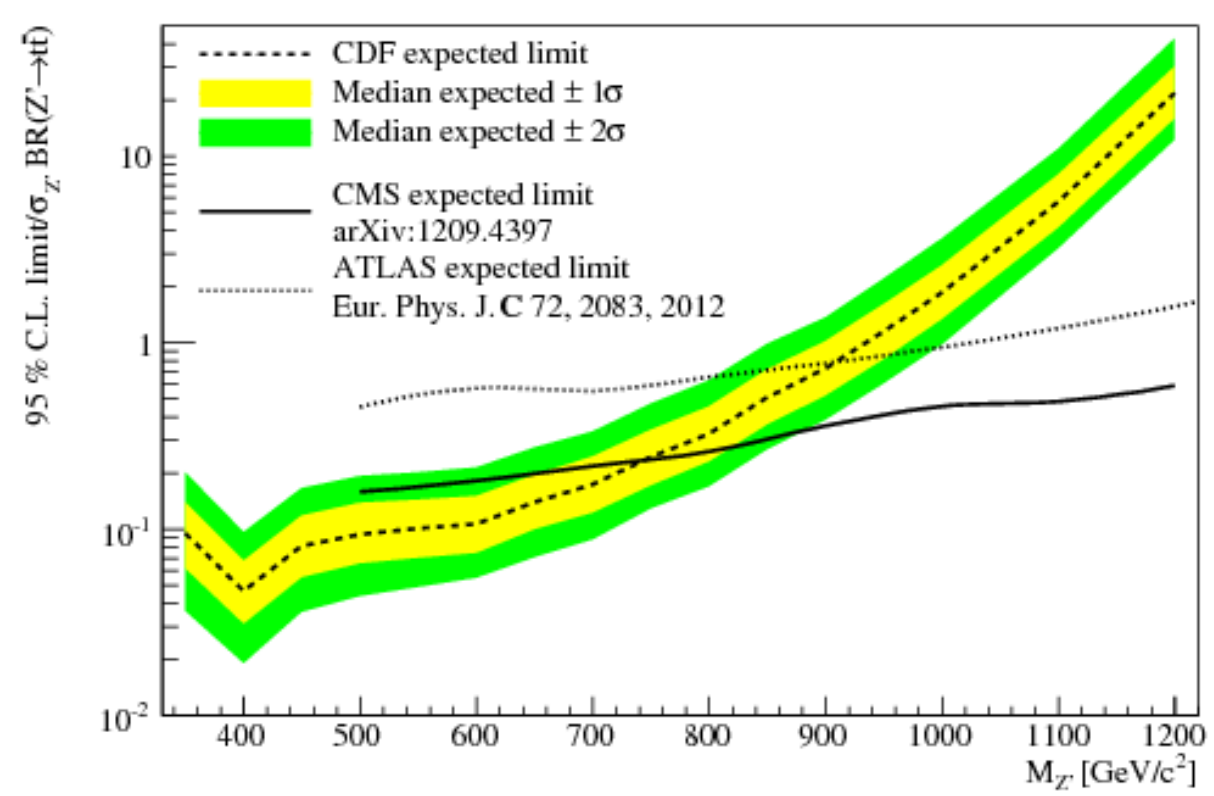

Figure 1.5 - Comparison between expected limits for CDF, CMS, and ATLAS in a search for a $Z^{\prime}$ boson resonance decaying to $t \bar{t}$. The $x$-axis shows the value of $M_{Z^{\prime}}$ and the y-axis shows the $95 \%$ C.L. observed limit normalized to the theoretical cross section in a benchmark model. From [36].

Preliminary studies indicate that CDF has higher sensitivity than the LHC also for a $W^{\prime}$ 
boson with mass just above the $t b$ mass, providing an opportunity to perform a search that is complementary to $Z^{\prime} \rightarrow t \overline{\text { }}$. As with the $Z^{\prime}$ boson search, the Tevatron and LHC experiments have previously searched for a $W^{\prime}$ boson, excluding a benchmark $W^{\prime}$ model for $\mathrm{W}^{\prime}$ boson masses below $2 \mathrm{TeV}$. In particular, in $2009 \mathrm{CDF}$ analyzed $1.9 \mathrm{fb}^{-1}$ of integrated luminosity to set limits on $W^{\prime}$ boson production by searching for an excess in events with a top quark and a bottom quark, with the top quark decaying to a $W$ boson and a bottom quark, and the $W$ boson decaying leptonically [37]. The invariant mass of the lepton, the missing transverse energy and the two $b$-jets in the final state was used to discriminate $W^{\prime}$ boson events from the background.

\subsubsection{Features of a $W^{\prime}$ Boson}

A boson with features of a $W^{\prime}$ boson that decays to $t b$ occurs in a variety of proposed beyond-the-Standard-Model (BSM) theories, manifesting itself as excitations of the $W$ boson in Kaluza-Klein extra-dimension models [38], as the techni- $\rho$ of technicolour theories [39], or as a bosonic partner in Little Higgs scenarios [40]. The classic $W^{\prime}$ boson is a simple extension of the Standard Model to a left-right symmetric group $\mathrm{SU}(2)_{L} \times$ $\mathrm{SU}_{R}(2) \times \mathrm{U}(1)$ [41], which has a right-handed charged boson $W_{R}^{\prime}$ with universal weak coupling strength and large mass. Searches in the $t b$ final state can probe models where the couplings are unknown, in particular when the $W_{R}^{\prime}$ leptonic channels are suppressed. The Lagrangian describing the $W^{\prime}$ coupling to fermions can be written as:

$$
\mathcal{L}=\frac{g}{\sqrt{2}} \bar{f}_{i} \gamma_{\mu}\left(a_{f_{i} f_{j}}^{L} P_{L}+a_{f_{i} f_{j}}^{R} P_{R}\right) W^{\prime \mu} f_{j}+\text { h.c. },
$$

where $P_{L, R}=\left(1 \mp \gamma^{5}\right) / 2$ are the projection operators, $g$ is the gauge coupling, and the $a_{f_{i} f_{j}}^{L, R}$ are arbitrary couplings that may differ for quarks and leptons.

To make a quantitative constraint, some general assumptions must be made. Our signal model assumes a $W^{\prime}$ boson with unknown mass, unspecified coupling strength to $t b$, and coupling to fermions with purely right- or left-handed chirality $\left(W_{R}^{\prime}\right.$ or $\left.W_{L}^{\prime}\right)$; this is referred to as a SM-like $W^{\prime}$ boson.

The $W^{\prime}$ boson is produced entirely via an s-channel process; contributions from the $t$ and $u$ channels are suppressed by the large $W^{\prime}$ boson mass. Like the Standard Model $W$ boson, the $W^{\prime}$ decays nearly instantaneously to a quark-antiquark pair, or to a lepton and a neutrino. The search described in this thesis is focused on events with a $W^{\prime}$ boson decaying to $t b$. 


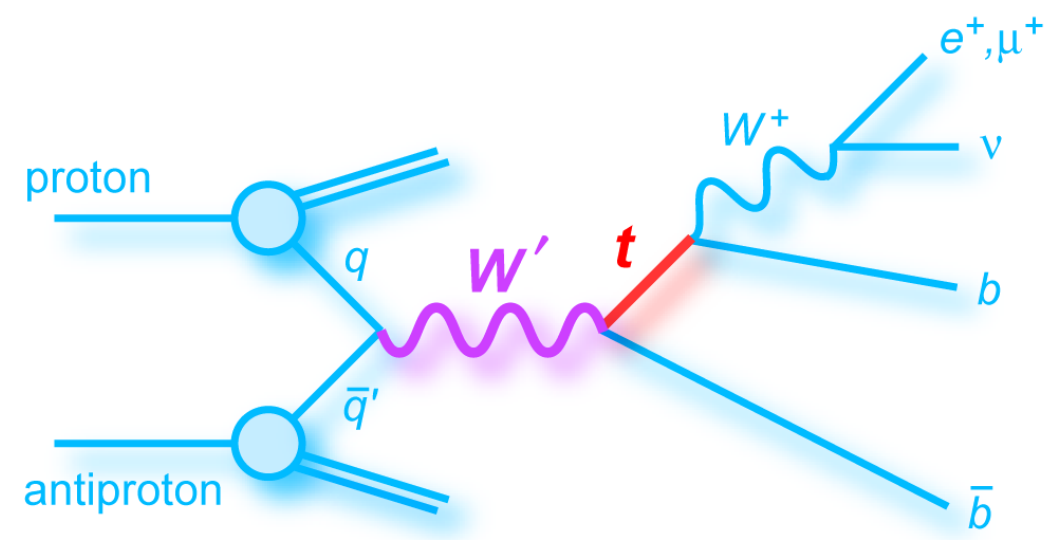

Figure 1.6 - Feynman diagrams for $W^{\prime}$-boson $s$-channel production and subsequent leptonic decay of the $W$ boson originating from the $t$ quark. 


\section{Chapter 2}

\section{The Experiment}

\subsection{The Tevatron Collider}

The Tevatron was a proton-antiproton accelerator located at the Fermi National Accelerator Laboratory. From 2001 to 2011 it produced $p \bar{p}$ collisions at a centre-of-mass energy of $\sqrt{s}=1.96 \mathrm{TeV}$. Protons and antiprotons collide at the two interaction points, where the Collider Detector at Fermilab (CDF) and D0 detector were installed. The Tevatron proton and antiproton beams were produced by a chain of accelerators, which performed proton and antiproton production, antiproton storage, and intermediate acceleration up to the injection into the Tevatron ring. The Tevatron accelerator complex is shown in Figure 2.1.

\subsubsection{The Proton Source}

The acceleration cycle of the proton beam begins with the production of negatively ionized hydrogen atoms $\left(H^{-}\right)^{1}$, which are initially accelerated to a kinetic energy of 750 $\mathrm{KeV}$ by a Cockroft-Walton electrostatic accelerator (the Preaccelerator). The $H^{-}$ions are then injected into the linear accelerator (LINAC), where they reach a kinetic energy of $400 \mathrm{MeV}$ by traveling through a $150 \mathrm{~m}$ long chain of radio-frequency (RF) accelerating cavities. Prior to being injected into the Booster synchrotron, the $\mathrm{H}^{-}$ions pass through a carbon foil which strips off the electrons. In the booster the protons are accelerated to 8 $\mathrm{GeV}$ by a number of RF cavities and are then transferred to another synchrotron, called the Main Injector ${ }^{2}$, which brings their energy up to $150 \mathrm{GeV}$. This is the final step before protons are injected into the Tevatron.

\footnotetext{
${ }^{1}$ At sub-relativistic energies one must accelerate negative ions rather than positive protons in order to avoid beam neutralization by electron capture.

${ }^{2}$ Completed in 1999 to operate in Tevatron Run II, which started in 2001, the Main Injector is located in a $3 \mathrm{~km}$ circumference tunnel, which also houses the antiproton Recycler and is approximately tangent to the Tevatron.
} 


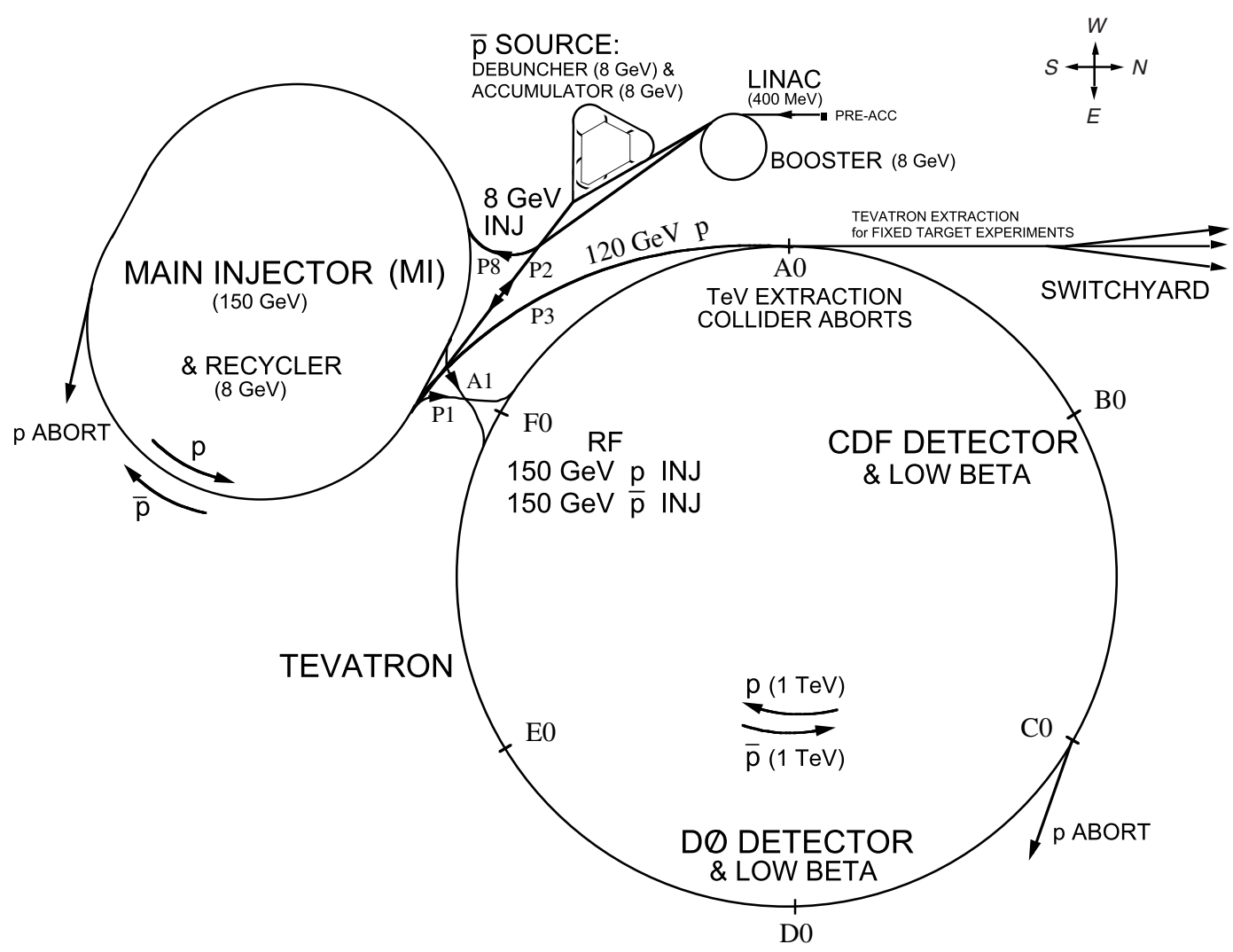

Figure 2.1 - The Fermilab accelerator system and the Tevatron collider.

\subsubsection{The Antiproton Source}

The production of the antiproton beam is significantly more complicated. The cycle starts with the extraction from the Main Injector of a $150 \mathrm{GeV}$ proton beam, which is directed onto a nickel alloy target. The collisions create a variety of different particles, among which are $\bar{p}$, produced with an efficiency of about $18 \bar{p} / 10^{6} p$. The particles, coming off the target at different angles, are focused into a beam by means of a magnetic lithium collection lens. In order to select only the antiprotons, the beam is sent through a pulsed magnet which acts as a charge-mass spectrometer. The emerging antiprotons, which have a bunch structure similar to that of the incident protons and a large momentum spread, are stored in the Debuncher, a storage ring where the $\bar{p}$ momentum spread is reduced via stochastic cooling ${ }^{3}$.

At the end of the debunching process the bunch structure is destroyed, resulting in a continuous beam of $8 \mathrm{GeV}$ antiprotons which are successively transferred to the Accumulator. The Accumulator is a triangle-shaped storage ring, housed in the same tunnel as the Debuncher, where the antiprotons are further cooled and stored. When the collected

\footnotetext{
${ }^{3}$ Stochastic cooling [42] is a technique used to reduce the transverse momentum and energy spread of a particle beam without any accompanying beam-loss. This is achieved by iteratively applying a feedback mechanism that senses the beam deviation from the ideal orbit with a set of electrostatic plates, processes and amplifies the signal, and transmits an adequately-sized synchronized correction pulse to another set of plates downstream.
} 
antiprotons saturate the Accumulator acceptance $\left(\sim 6 \times 10^{11}\right.$ antiprotons), they are transferred to the Recycler ${ }^{4}$, an $8 \mathrm{GeV}$ fixed-energy storage ring with a larger acceptance, made of permanent magnets and placed in the Main Injector tunnel. In the Recycler the size and spread of the antiproton beam is further shrunk by the electron cooling process: in a straight section of the Recycler a beam of electrons travels close to the antiprotons at the same velocity, absorbing energy from the antiprotons. When a current sufficient to create 36 bunches with the required density is available, the $\bar{p}$ are injected into the Main Injector where they are accelerated to a momentum of $150 \mathrm{GeV}$. At this point the antiprotons are ready to be injected into the Tevatron in the opposite direction to the previously stored $150 \mathrm{GeV}$ proton beam.

\subsubsection{The Tevatron Ring}

The Tevatron is a $1 \mathrm{~km}$-radius superconducting synchrotron that accelerates particles from $150 \mathrm{GeV}$ to $980 \mathrm{GeV}$. The proton and antiproton beams circulate in opposite directions in the same beam pipe. Electrostatic separators produce a strong electric field that keeps the two beams away from each other except at the collision points. The beam is steered by 77 super-conducting dipole magnets with a maximum magnetic field of $4.2 \mathrm{~T}$ and focused by 240 quadrupole magnets. A cryogenic system consisting of liquid nitrogen followed by liquid helium cools the Tevatron magnets to $4.2^{\circ} \mathrm{K}$, at which temperature the niobium-titanium alloy of the magnet coils becomes superconducting. The process of injecting particles into the machine, accelerating them, and initiating collisions, is referred to as a shot. It starts with the injection from the Main Injector of $150 \mathrm{GeV}$ protons, two bunches at a time. Once the proton beam is in the machine, groups of four antiprotons bunches are mined from the Recycler, accelerated to $150 \mathrm{GeV}$ in the Main Injector and injected into the Tevatron. After the RF cavities accelerate the beams to 980 $\mathrm{GeV}$, electrostatic separators switch polarity and cause the beams to collide at two points. Each interaction point lies at the centre of a particle detector: D0 named after its location in the Tevatron, and CDF, located at the Tevatron optic point B0.

Subsequently, beams are scraped with remotely-operated collimators to remove the beam halo and, as soon as the beam conditions are stable, the experiments begin to take data. A continuous period of collider operation with the same proton and antiproton beams is called a store.

\footnotetext{
${ }^{4}$ Antiproton availability is the most limiting factor at the Tevatron for attaining high luminosities: keeping a large antiproton beam inside the Recycler has been one of the most significant engineering achievements in Run II.
} 
Table 2.1 - Accelerator parameters for Run II [43].

\begin{tabular}{lc}
\hline \hline Parameter & Run II value \\
\hline number of bunches $\left(N_{b}\right)$ & 36 \\
revolution frequency $[\mathrm{MHz}](f)$ & 1.7 \\
rms bunch length $[\mathrm{m}] \sigma_{l}$ & 0.26 \\
bunch spacing [ns] & 396 \\
protons/bunch $\left(N_{p}\right)$ & $2.7 \times 10^{11}$ \\
antiprotons/bunch $\left(N_{\bar{p}}\right)$ & $3.0 \times 10^{10}$ \\
total antiprotons & $1.1 \times 10^{12}$ \\
$\beta^{*}[\mathrm{~cm}]$ & 35 \\
\hline \hline
\end{tabular}

\subsubsection{Luminosity and Tevatron Performance}

The performance of a collider is evaluated in terms of two key parameters: the available centre-of-mass energy, $\sqrt{s}$, and the instantaneous luminosity, $\mathcal{L}$. The former defines the accessible phase-space for the production of final state particles. The latter is defined as the number of crossing particles per unit area and per unit time [crossing particles $\left./\left(\mathrm{cm}^{2} \cdot \mathrm{s}\right)\right]$. In the absence of a crossing angle or position offset of the beams, the luminosity at CDF or D0, is given by the expression:

$$
\mathcal{L}=\frac{f N_{b} N_{p} N_{\bar{p}}}{2 \pi\left(\sigma_{\bar{p}}^{2}+\sigma_{\bar{p}}^{2}\right)} F\left(\frac{\sigma_{l}}{\beta^{*}}\right),
$$

where $f$ is the revolution frequency, $N_{b}$ is the number of bunches, $N_{p(\bar{p})}$ is the number of protons (antiprotons) per bunch, and $\sigma_{p(\bar{p})}$ is the transverse proton (antiproton) beam size at the interaction point. $F$ is a form factor with a complicated dependence on the beta function value at the interaction point $\left(\beta^{* 5}\right)$, and the bunch length, $\sigma_{l}$.

Table 2.1 shows the design Run II accelerator parameters, while Figure 2.2 shows the evolution of the integrated luminosity, defined as $L=\int \mathcal{L} d t$, and of the instantaneous luminosity at the start of Tevatron stores. The steady increase of the integrated and instantaneous luminosities demonstrate the outstanding performance of the accelerator.

The Tevatron program was terminated on September 30, 2011. During Run II the Tevatron delivered $12 \mathrm{fb}^{-1}$ integrated luminosity to each experiment, almost $10 \mathrm{fb}^{-1}$ of which were collected by each of the CDF and D0 detectors.

\subsection{The CDF II Detector}

The CDF II detector [44] is an approximately azimuthally and forward-backward symmetric apparatus designed to study the $p \bar{p}$ collisions at the B0 interaction point of the

\footnotetext{
${ }^{5}$ The beta function represents a measure of the local transverse beam size. The value of the beta function at an interaction point is referred to as $\left(\beta^{*}\right)$.
} 

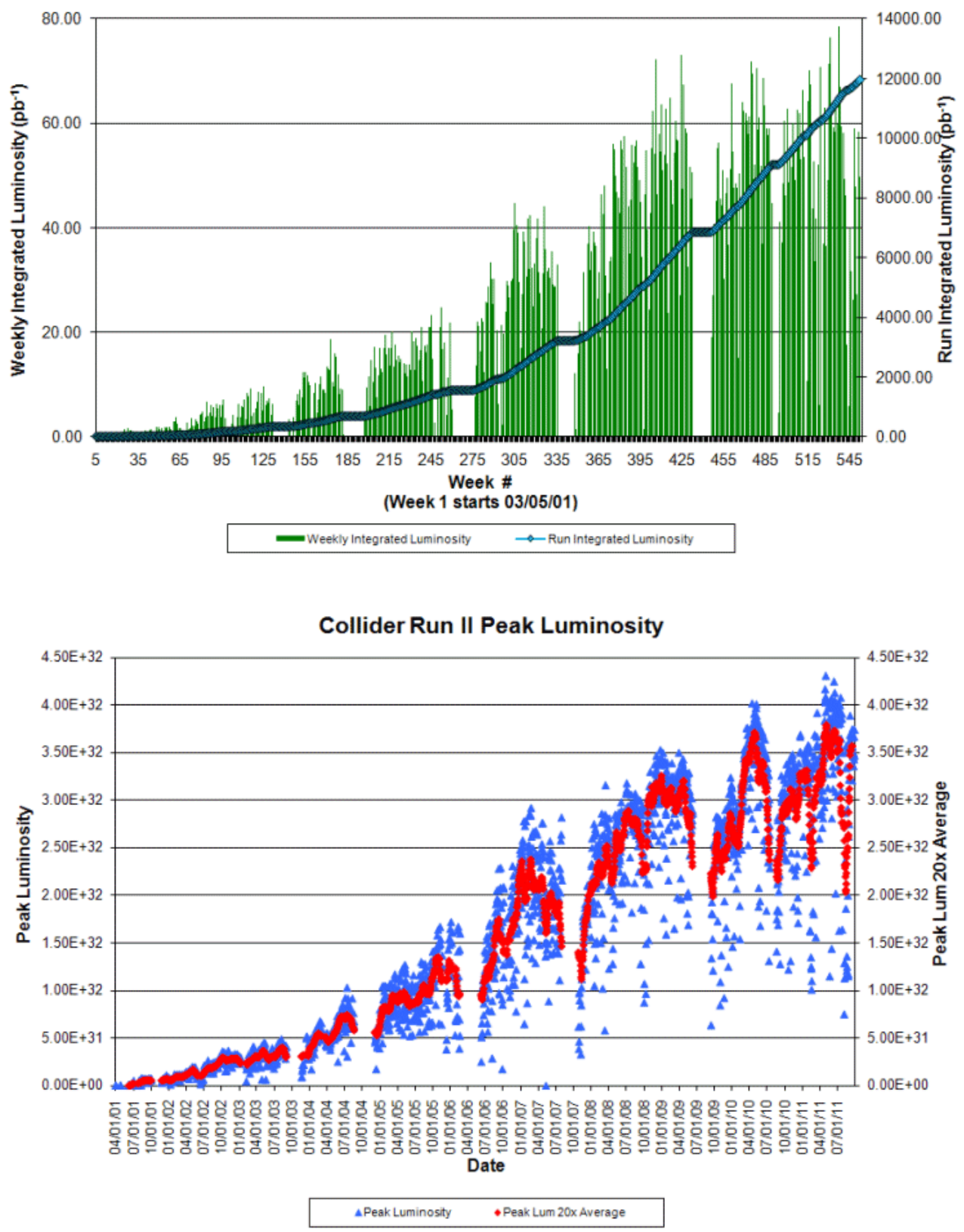

Figure 2.2 - Tevatron integrated luminosity as a function of Run II week number (top) and peak luminosity as a function of calendar date (bottom). Empty regions correspond to Tevatron shut-down periods.

Tevatron.

The CDF II sub-detectors (Figure 2.3) are:

- a tracking system, which comprises three silicon microstrip trackers (L00, SVX and ISL) and an open-cell drift chamber (COT) inside a superconducting solenoid that provides a constant $1.4 \mathrm{~T}$ magnetic field parallel to the beam direction, with the purpose of bending the trajectories of charged particles to allow the determination 


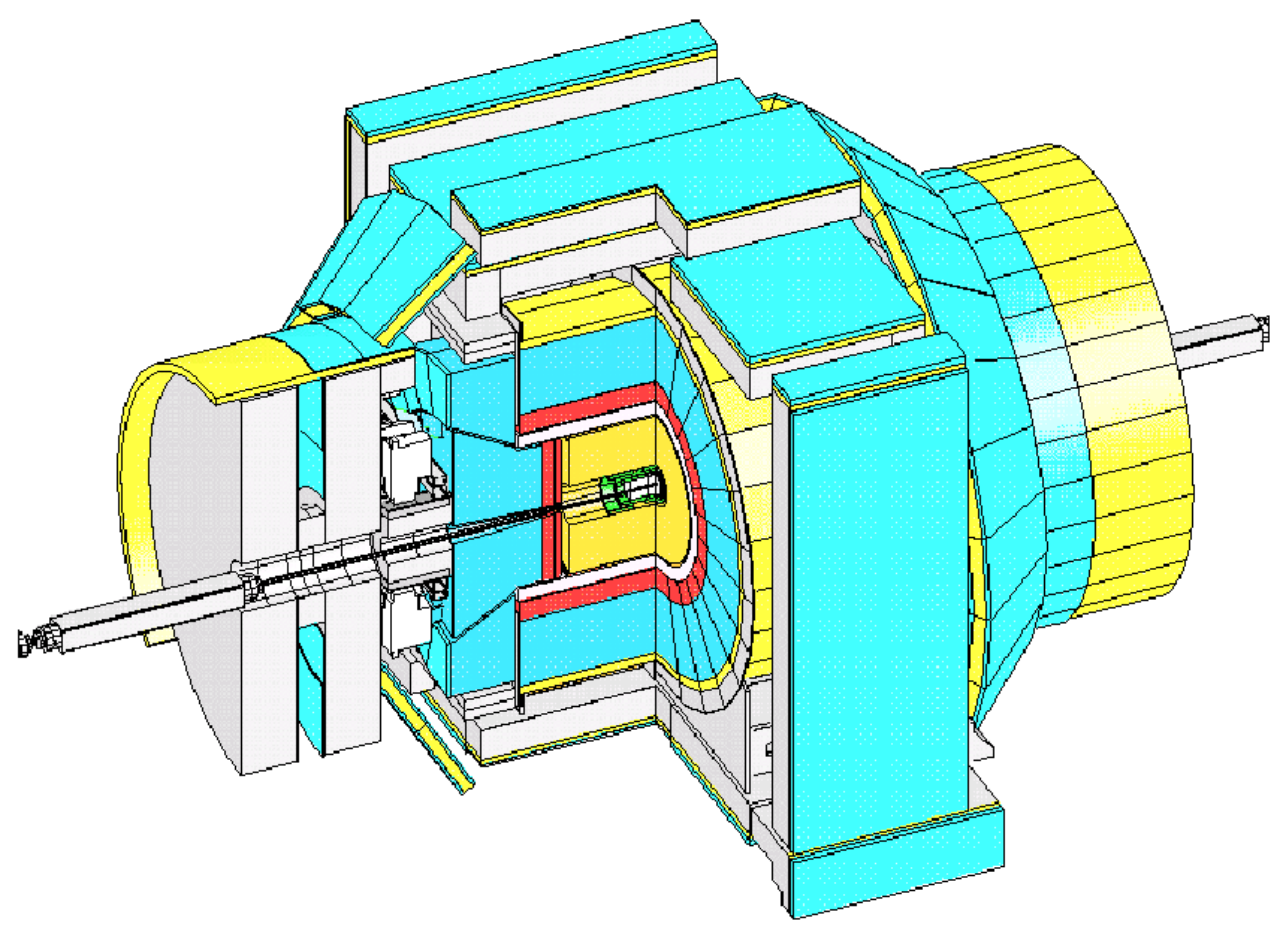

Figure 2.3 - Isometric view of the CDF II detector.

of their momenta and the signs of their charges;

- a time-of-flight system (TOF), located outside the COT, for measuring the masses of charged particles with momenta up to $2 \mathrm{GeV} / c$;

- a calorimeter system, with the purpose of measuring the energies of charged and neutral hadrons, and electrons and photons ${ }^{6}$;

- muon chambers and scintillators, used to track and identify muons that pass through the calorimeters and interact as minimum ionizing particles;

- luminosity monitors, for the instantaneous luminosity measurement, necessary to derive cross sections from event yields.

\subsubsection{Coordinates System and Conventions at CDF}

CDF adopts a right-handed Cartesian coordinate system with origin at the nominal interaction point, coincident with the centre of the drift chamber. The positive $z$-axis lies along the nominal beam-line and has the direction of the proton beam (eastward). The $x$ $y$ plane is therefore perpendicular to the beam-line, with the $y$-axis pointing upwards and the $x$-axis in the horizontal plane, pointing radially outward with respect to the centre of

\footnotetext{
${ }^{6}$ Muon momentum is measured in the tracker.
} 
the accelerator ring. A cylindrical $(r, \phi, z)$ coordinate system is particularly convenient to describe the detector geometry, where

$$
r=\sqrt{x^{2}+y^{2}} \text { and } \phi=\tan ^{-1} \frac{y}{x}
$$

A momentum-dependent coordinate, named rapidity, is also commonly used in highenergy physics for its transformation properties under Lorentz boosts. The rapidity is defined as

$$
y=\frac{1}{2} \ln \frac{E+p_{z}}{E-p_{z}}
$$

where $E$ is the energy and $p_{z}$ is the $z$ component of the particle momentum. Rapidity intervals are Lorentz invariant. In the relativistic limit, or when the mass of the particle is negligible, rapidity depends only upon the production angle of the particle with respect to the beam axis, $\theta=\tan ^{-1} \frac{\sqrt{x^{2}+y^{2}}}{z}$. This approximation is called pseudorapidity $\eta$ and is defined as

$$
y \stackrel{p \gg m}{\rightarrow} \eta=-\ln \left(\tan \frac{\theta}{2}\right)
$$

A value of $\theta=90^{\circ}$, perpendicular to the beam axis, corresponds to $\eta=0$.

Since the event-by-event longitudinal position of the interaction is distributed around the nominal interaction point with a $30 \mathrm{~cm}$ RMS width, sometimes a distinction between the detector pseudorapidity (usually indicated with $\eta_{\text {det }}$ ), measured with respect to the $(0,0,0)$ nominal interaction point, and the event pseudorapidity $(\eta)$, which is measured with respect to the $z$ position of the actual event vertex, is considered.

The spatial separation between particles in the detector is commonly given in terms of a Lorentz invariant variable defined as:

$$
\Delta R=\sqrt{\Delta \phi^{2}+\Delta \eta^{2}}
$$

Because of energy and momentum conservation in the transverse plane, other quantities useful to describe the kinematics of $p \bar{p}$ interactions are the transverse momentum and the transverse energy, defined as $p_{T}=p \sin \theta$ and $E_{T}=E \sin \theta$, respectively.

\subsection{The Tracking System}

The innermost silicon detectors provide a precise determination of the track impact parameter and the $z$ coordinate at production, whereas the drift chamber has excellent resolution on the transverse momentum, $\phi$ and $\eta$. The combined information of the tracking detectors provides very accurate measurements of the helical paths of charged particles inside the detector. We will describe this system starting from the devices closest to the beam and moving outwards (see Figure 2.4). 

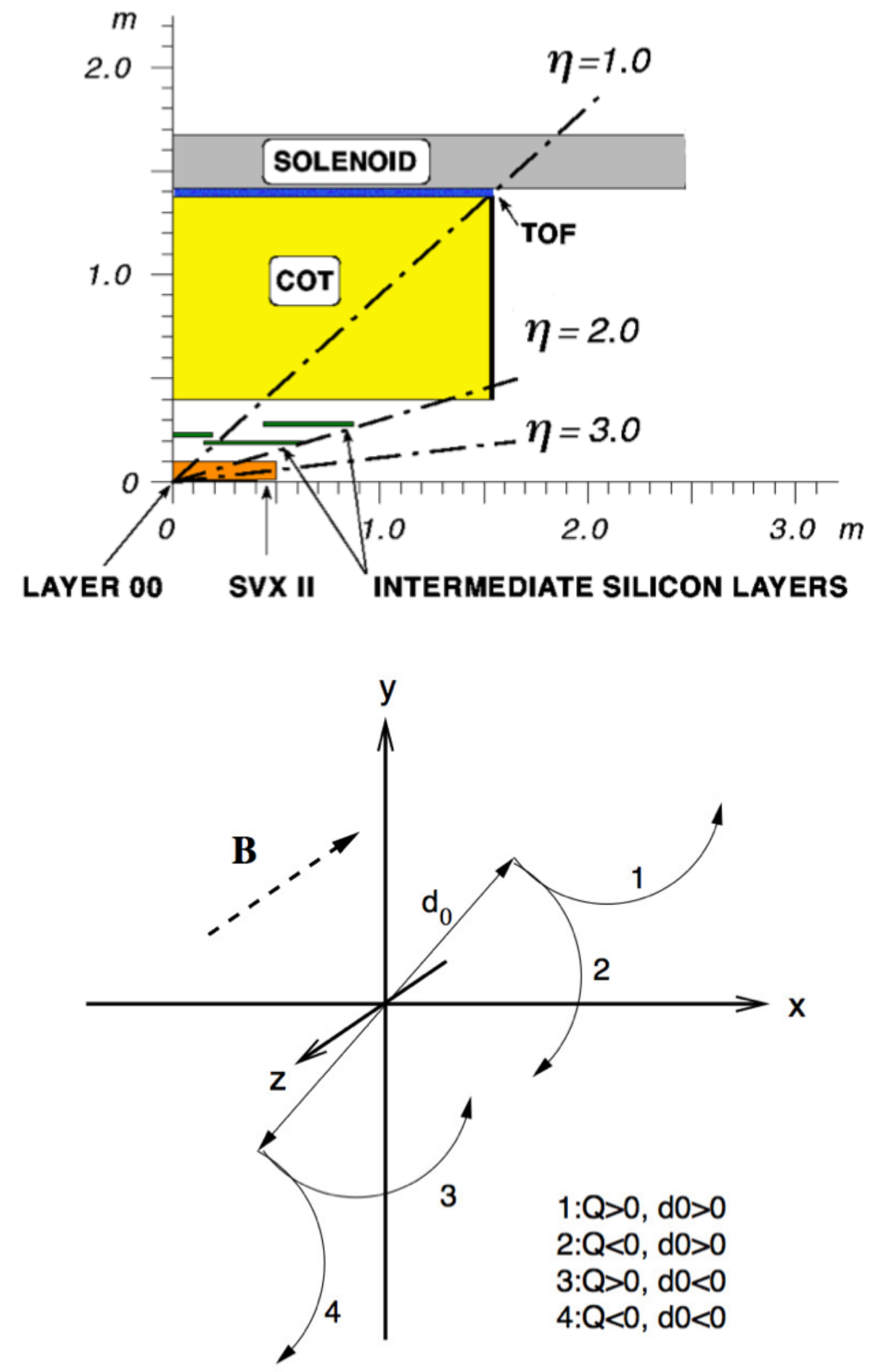

Figure 2.4 - (top) The CDF II tracker layout showing the different subdetector systems. (bottom) Schematic drawing of the impact parameter $d_{0}$. The sign of the impact parameter is defined as positive or negative with reference to the direction of the track momentum vector (in the $x, y$ quadrant in the drawing). The sign of the impact parameter is negative for tracks 2 and 3 , positive for tracks 1 and 4 . 


\subsubsection{The Silicon Tracker}

The full CDF II silicon detector is composed of three approximately cylindrical coaxial subsystems: the Layer 00 (L00), the silicon vertex detector (SVX) and the intermediate silicon layers (ISL).

Silicon sensors operate as reverse-biased $p$ - $n$ junctions. By segmenting the $p$ or $n$ side of the junctions into strips and reading out the charge deposition separately on every strip, the point of traversal of a charged particle is measured. At CDF the typical distance between two strips is about $60 \mu \mathrm{m}$. There are two types of microstrip detectors: single-sided and double-sided. In single-sided detectors only the $p$-side of the junction is segmented into strips, while double-sided detectors have both sides of the junction segmented into strips. Single-sided sensors have strips parallel to the $z$ direction and provide only $\phi$ position measurements, while double-sided detectors have strips at an angle (stereo angle) with respect to the $z$ direction on one side and, therefore, provide also information on the particle position along $z$.

L00 [45] is a $90 \mathrm{~cm}$-long, radiation-hard assembly of single-sided silicon detectors, structured in longitudinal strips. The detector tiles are mounted directly on the beam pipe at 1.35 or $1.62 \mathrm{~cm}$ (depending on their azimuth) from the beam axis. The detector support structure is carbon fiber with an integrated cooling system. Being so close to the beam, L00 allows a resolution of 25-30 $\mu \mathrm{m}$ on the impact parameter of tracks of moderate $p_{T}$, providing a powerful handle to identify long-lived hadrons containing a $b$ quark.

SVX [45] is composed of three $29 \mathrm{~cm}$-long cylindrical barrels, radially organized in five layers of double-sided silicon wafers extending from $2.5 \mathrm{~cm}$ to $10.7 \mathrm{~cm}$ (see Figure 2.5). Each barrel is segmented into 12 wedges, each covering $\sim 30^{\circ}$ in $\phi$. The double-sided structure of the wafers allows a three dimensional position measurement: one side of the wafer has axial strips (parallel to the beam), the other one has either $90^{\circ}$ strips (perpendicular to the beam) or $1.2^{\circ}$ stereo strips (at small angle with respect to the beam). This detector provides position information with $12 \mu \mathrm{m}$ resolution on a single hit and some $d E / d x$ ionization information.

ISL [47] consists of two layers of double-sided silicon wafers, similar to those of SVX. Two lateral sectors are assembled in a two-fold telescope with planes at a radial distance of $22 \mathrm{~cm}$ and $29 \mathrm{~cm}$ from the beam-line and covering $1<|\eta|<2$. One single central layer is located at $r=22 \mathrm{~cm}$, covering $|\eta|<1$. The two ISL layers are important to increase the tracking coverage in the forward region, where the COT coverage is limited, and to improve the matching between SVX and COT tracks.

The combined resolution of the CDF inner tracker on the point of origin for high momentum tracks is $\sim 12 \mu \mathrm{m}$ for the transverse impact parameter and $\sim 70 \mu \mathrm{m}$ along the $z$ direction. All silicon detectors are used in the off-line track reconstruction algorithms [48], 

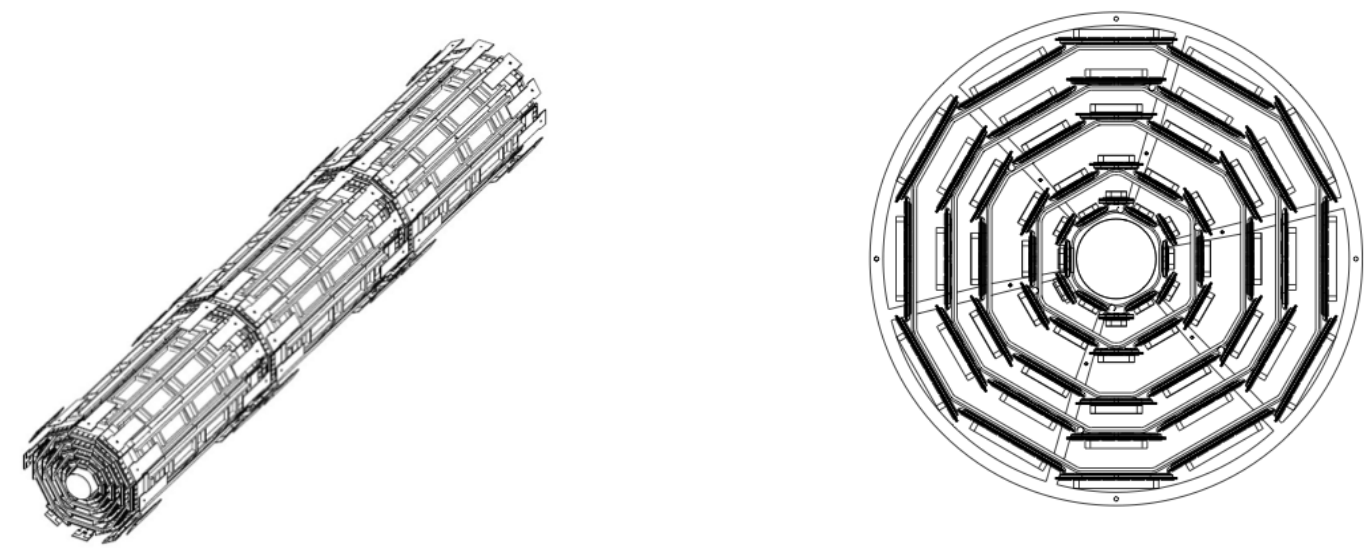

Figure 2.5 - The SVX silicon detector: on the left, a three-dimensional view of the detector shows the barrel structure along the beam axis; on the right, the transverse plane section shows in detail the layer sequence. The innermost layer L00 is supported by the beam pipe.

while the SVX plays a crucial role also in the on-line track reconstruction of the trigger system. The CDF trigger employs an innovative processor, the Silicon Vertex Trigger (SVT) [49], which uses the SVX information to measure the track impact parameter on-line with a resolution of $<20 \mu \mathrm{m}$, allowing the identification of secondary vertices, displaced from the primary interaction point, as typically produced by $B$-hadron decays.

\subsubsection{Central Outer Tracker}

Surrounding the silicon detector is the Central Outer Tracker (COT) [50]. It is a $3.1 \mathrm{~m}-l$ long cylindrical drift chamber, coaxial with the beam, and covers the radial range from 40 to $137 \mathrm{~cm}$ for $|\eta|<1$. The COT contains 96 sense wire layers, which are radially grouped into 8 superlayers, as shown in the end plate slot structure in Figure 2.6. Each superlayer is divided in $\phi$ into supercells, and each supercell has 12 alternating sense and field-shaping wires. The maximum drift distance is approximately the same for all superlayers. To achieve this, the number of supercells in a given superlayer scales approximately with the radius of the superlayer. The entire COT contains 30240 sense wires. Approximately half the wires run along the $z$ direction (axial wires), the other half are strung at a small stereo angle $\left(2^{\circ}\right)$ with respect to the $z$ direction (stereo wires). The combination of axial and stereo information allows the measurement of the track $z$ positions and therefore a three-dimensional reconstruction of tracks. Particles originating from the interaction point and having $|\eta|<1$ pass through all 8 COT superlayers. The COT is filled with an argon-ethane gas mixture and isopropyl alcohol. The mixture is chosen in order to have a constant drift velocity, approximately $50 \mu \mathrm{m} / \mathrm{ns}$, across the cell width. The maximum electron drift time is approximately $100 \mathrm{~ns}$. Due to the magnetic field present in the COT, electrons drift at a Lorentz angle of $35^{\circ}$. The supercells are tilted by $35^{\circ}$ with respect to the radial direction to compensate for this effect and make the drift path perpendicular to the radial direction. 

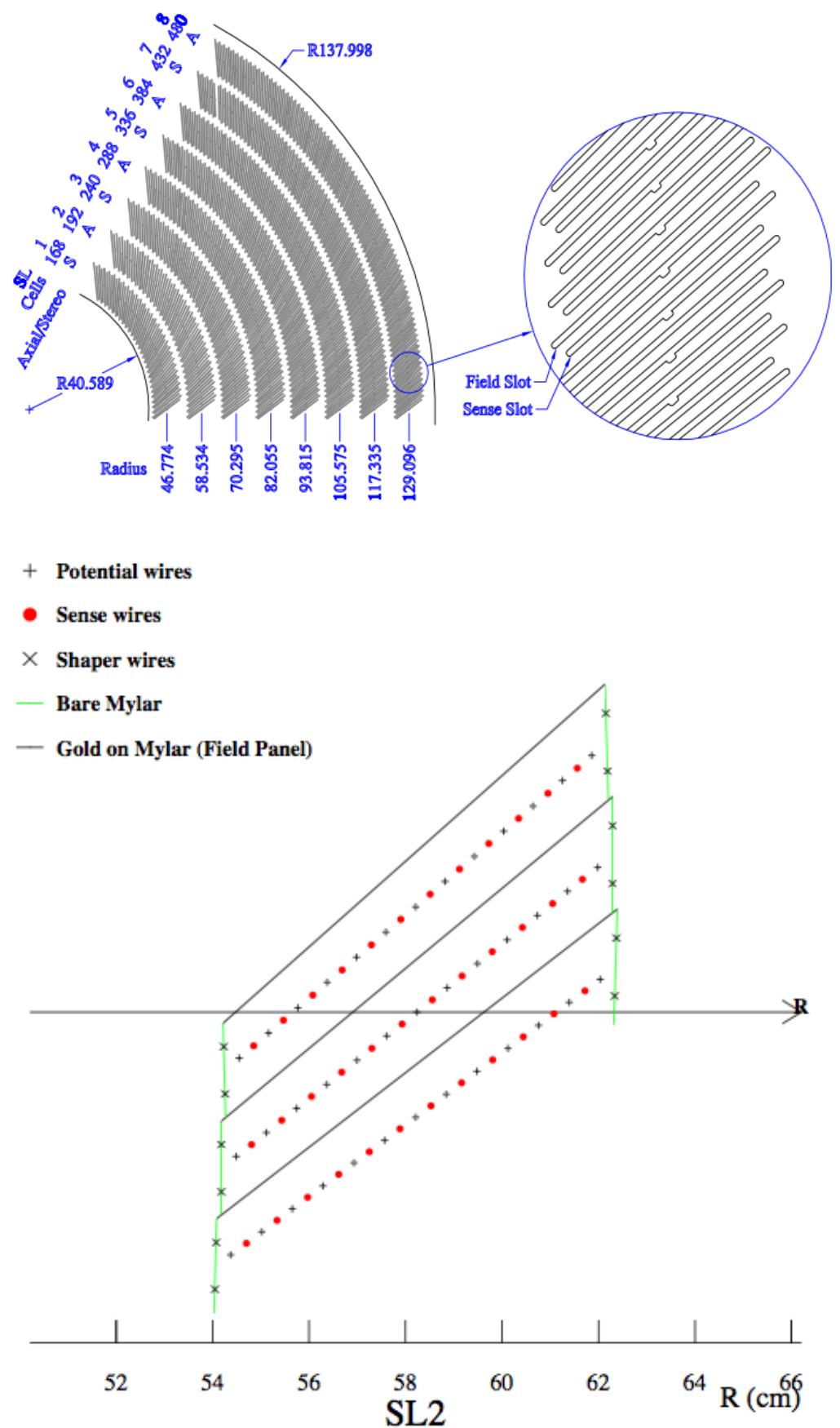

Figure 2.6 - (top) $60^{\circ}$ section of the COT end-plate. For each super-layer the total number of cells, the wire orientation (axial or stereo) and the average radius in $\mathrm{cm}$ are shown. The enlargement shows in detail the slot where the wire planes (sense and field) are installed. (bottom) Cross-section of three axial cells in super-layer 2; the arrow indicates the radial direction.

The hit position resolution in the $r-\phi$ plane is about $140 \mu \mathrm{m}$. Tracking algorithms are utilized to reconstruct particle trajectories (helices) that best fit to the observed hits. The reconstructed trajectories are referred to as tracks. Particle momentum and charge sign are determined from the bending of the tracks in the magnetic field. The COT hits are 
also processed on-line by the extremely fast tracker (XFT), which reconstructs the tracks at Level 1 of the trigger system, (Section 2.7). The transverse momentum resolution of off-line tracks with only COT hits, measured by analyzing cosmic ray tracks traversing the beam-beam source as two separate out-going tracks, is

$$
\frac{\sigma_{p_{T}}}{p_{T}^{2}}=0.0017(\mathrm{GeV} / c)^{-1}
$$

for tracks with $p_{T}>2 \mathrm{GeV} / c[51]$.

\subsubsection{Time of Flight}

The CDF II time-of-flight (TOF) detector [52] lies just outside the tracking system, supported on the inner wall of the solenoid (Figure 2.4). It is a barrel of $3 \mathrm{~m}$ long scintillators bars located at $140 \mathrm{~cm}$ from the beam line with a total of 216 bars, each covering $1.7^{\circ}$ in $\varphi$ and the pseudorapidity range $|\eta|<1$. Light is collected by photomultipliers at both ends of the bar. A single-hit position in the TOF is determined by comparing the arrival times of the signals at the two photomultipliers. Particle identification is achieved by measuring the time of arrival of a charged particle at the scintillators with respect to the collision time. By combining the measured time-of-flight $T$, the momentum $p$, and the path length $L$, the mass of the particle can be estimated.

$$
m=\frac{p}{c} \sqrt{\frac{1}{\beta^{2}}-1}=\frac{p}{c} \sqrt{\left(\frac{c T}{L}\right)^{2}-1},
$$

where the momentum and path length are precisely measured by the tracking system. For the TOF measurement the collision time must be known and this is determined to a precision of 50 ps using TOF measurements of all tracks from the collision. The design resolution of the time-of-flight measurement of a single track is $\approx 120 \mathrm{ps}$ and provides at least two standard-deviation separation between $K^{ \pm}$and $\pi^{ \pm}$for momenta $p<1.6 \mathrm{GeV} / c$.

\subsection{Calorimeter}

The calorimeter system is one of the main subdetectors of CDF II (the others are the muon and tracking systems). It has been designed to measure the energies and positions of neutral and charged particles leaving the tracking region. In particular, it is devoted to jet, electron, and photon reconstruction, and is also used to measure the momentum imbalance due to neutrinos. Particles hitting the calorimeter can be divided in two classes according to their main interaction with matter: electromagnetically interacting particles, such as electrons and photons, and hadronically interacting particles, such as mesons or baryons. To detect these two classes of particles, two different calorimetric sections are 
used: an inner electromagnetic section, and an outer hadronic section, both providing coverage for $|\eta|<3.64$.

In order to supply information on particle position, the calorimeter is also segmented into azimuthal wedges and polar-angle towers, projected toward the geometrical centre of the detector. Each tower consists of alternating layers of passive material and scintillator tiles. Depending on the angle, the light is read out by wave-shifting plates, fingers or wires coupled to the scintillators and carried to photomultipliers by means of light guides. The central sector of the calorimeter, covering the region $|\eta|<1$. , was recycled from Run I, while brand new calorimeters (called plug calorimeters) were built to cover the forward and backward regions of the CDF II calorimeter system. Figure 2.7 shows the main components of the calorimeter system.

\subsubsection{The Central Calorimeter}

Excluding upgrades on the readout electronics required to cope with the increased collision rate, the central calorimeter is the same as in Run I ${ }^{7}$. The central electromagnetic calorimeter (CEM) is segmented in $\Delta \eta \times \Delta \phi=0.11 \times 15^{\circ}$ projective towers consisting of 31 alternate layers of lead and scintillator, for a total material depth of $19 \mathrm{X}_{0}{ }^{8}$. The central and end-wall hadronic calorimeters (CHA and WHA respectively), whose geometric tower segmentation matches the one used for the CEM, use 32 steel layers of $2.5 \mathrm{~cm}$ thickness (5 $\mathrm{cm}$ in the wall), each sampled by a $1 \mathrm{~cm}$ thick acrylic scintillator. The total thickness of the hadronic section corresponds to 4.5 interaction lengths $\left(\lambda_{0}\right)^{9}$. A perspective view of a central electromagnetic calorimeter module (wedge) is shown in Figure 2.7, where both the arrangement in projective towers and the light pipes of the electromagnetic compartment are shown.

The projective geometry has been used in order to better measure the overall transverse momentum of particles in an event. The imbalance in total transverse momentum is a measure of the transverse momentum carried away by neutrinos. For each tower the transverse energy $E_{T}$ is defined as $E_{T}=E \sin \theta$, where $E$ is the energy detected by the tower and $\theta$ is the angle between the collision point and the projective tower direction.

\footnotetext{
${ }^{7} \mathrm{CDF}$, from 1992 to 2004, used embedded layers of gas proportional chambers in the central calorimeter to improve the identification of electrons and photons (central preshower (CPR) and central crack (CCR) detectors). Late in 2004 the CDF central preshower and crack detector upgrade system was installed. This system consists of scintillator tiles with wavelength-shifting fibers, clear-fiber optical cables, and multianode photomultiplier tubes.

${ }^{8}$ The radiation length $X_{0}$ describes the characteristic amount of matter transversed by high energy electrons to lose by bremsstrahlung all but $1 / e$ of their energy; this is equivalent to $7 / 9$ of the length of the mean free path of high energy photons before undergoing $e^{+} e^{-}$pair production. The average energy loss per distance travelled through the traversed material due to bremsstrahlung for an electron of energy $E$ is related to the radiation length by $\left(\frac{d E}{d x}\right)_{b r e m}=-\frac{E}{X_{0}}$

${ }^{9}$ An interaction length is the average distance that a particle will travel before interacting with a nucleus: $\lambda=\frac{A}{\rho \sigma N_{A}}$, where $A$ is the atomic weight, $\rho$ is the material density, $\sigma$ is the cross section and $N_{A}$ is Avogadro's constant.
} 

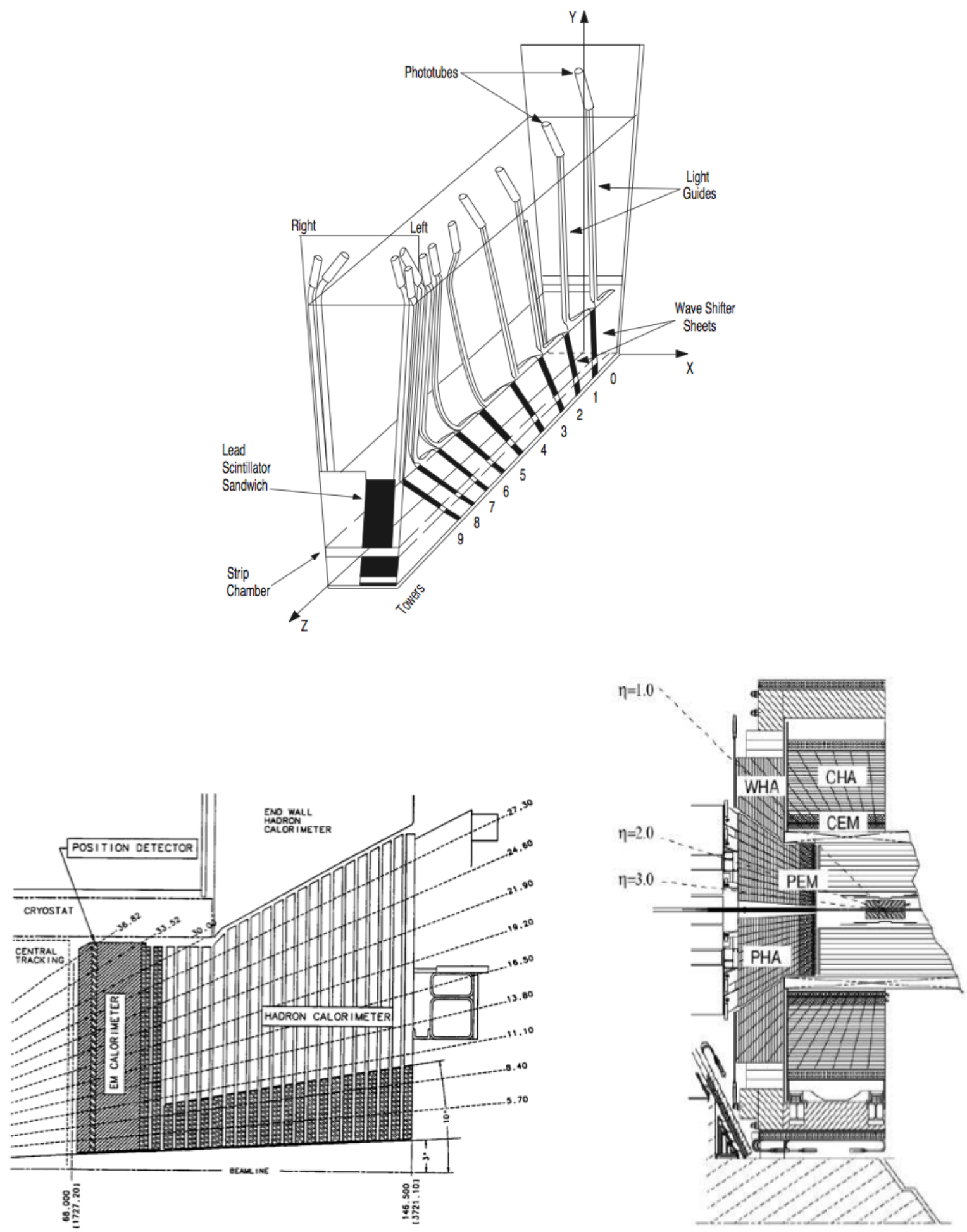

Figure 2.7 - (top) Artist's view of a central calorimeter wedge showing the pipes collecting light from the front electromagnetic towers; (bottom left) cut view of the plug; (bottom right) elevation view of the detector showing the components of the calorimeter system. 
Two position detectors complement each wedge of CEM:

- The central electromagnetic shower chamber (CES) is a two-dimensional strip-wire chamber located at the depth of the maximum electromagnetic shower $\left(\sim 5.9 X_{0}\right)$. It measures the charge deposition, providing information on their shower energy and position with a finer azimuthal segmentation than the calorimeter towers. This results in an increased purity of electromagnetic object reconstruction. The CES also measures the shower shape, which is used to distinguish electrons and photons from hadrons.

- The central pre-radiator (CPR) consists of two wire chamber modules placed immediately in front of the calorimeter. It acts as a pre-shower detector by using the tracker and the solenoid coil material as radiators, providing a useful tool for distinguishing hadrons from electrons and photons.

Table 2.2 summarizes the basic parameters of the calorimeter detectors. The energy resolution for each calorimeter section was measured in the test beam and, for a perpendicularly incident beam, can be parametrized as

$$
\frac{\sigma}{E}=\frac{\sigma_{1}}{\sqrt{E}} \oplus \sigma_{2}
$$

where the first term comes from the sampling fluctuations and the photostatistics of the PMTs (stochastic term), and the second term comes from the non-uniform response of the calorimeter (constant term). The symbol $\oplus$ indicates addition in quadrature.

\subsubsection{The Plug Calorimeter}

The Run II plug calorimeter covers the $|\eta|$ region from 1.1 to 3.64. Both electromagnetic and hadronic sectors are divided in $12 \eta$-regions, with $\Delta \eta$ ( $\eta$-width) increasing from 0.10 to 0.64 with increasing pseudorapidity. Each region is segmented in 48 or 24 (for $|\eta|>2.11$ or $|\eta|<2.11$ respectively) projective towers in azimuth. The actual size of these towers was chosen to have optimal separation of electron showers from the narrow forward hadron jets. Projective towers consist of alternating layers of absorbing material (lead and iron for electromagnetic and hadronic sectors, respectively) and scintillator tiles. The first layer of the electromagnetic tiles is thicker (10 $\mathrm{mm}$ instead of $6 \mathrm{~mm})$ and made of material with higher photon yield. It acts as a preshower detector.

\subsection{The Muon Chambers}

Most particles produced in the primary interaction or in subsequent decays have a very high probability of being absorbed in the calorimeter system. Muons are an exception. 
Table 2.2 - Summary of the main parameters of the CDF II calorimeter system.

\begin{tabular}{clclcl}
\hline \hline Calorimeter & CEM & CHA & WHA & PEM & PHA \\
\hline Absorber & Lead & Steel & Steel & Lead & Iron \\
Segmentation $(\eta \times \phi)$ & $0.1 \times 15^{\circ}$ & $0.1 \times 15^{\circ}$ & $0.1 \times 15^{\circ}$ & $(0.1-0.6) \times\left(7.5-15^{\circ}\right)$ & $(0.1-0.6) \times\left(7.5-15^{\circ}\right)$ \\
Num. Towers $(\eta \times \phi)$ & $20 \times 24$ & $9 \times 24$ & $6 \times 24$ & $12 \times 24(48)$ & $11 \times 24(48)$ \\
Thickness & $19 X_{0}, 1 \lambda_{0}$ & $4.7 \lambda_{0}$ & $4.7 \lambda_{0}$ & $23 X_{0}, 1 \lambda_{0}$ & $6.8 \lambda_{0}$ \\
Resolution $(\%)$ & $14 \sqrt{E_{T}} \oplus 2$ & $50 \sqrt{E_{T}} \oplus 3$ & $75 \sqrt{E_{T}} \oplus 4$ & $16 \sqrt{E} \oplus 1$ & $80 \sqrt{E} \oplus 5$ \\
\hline \hline
\end{tabular}

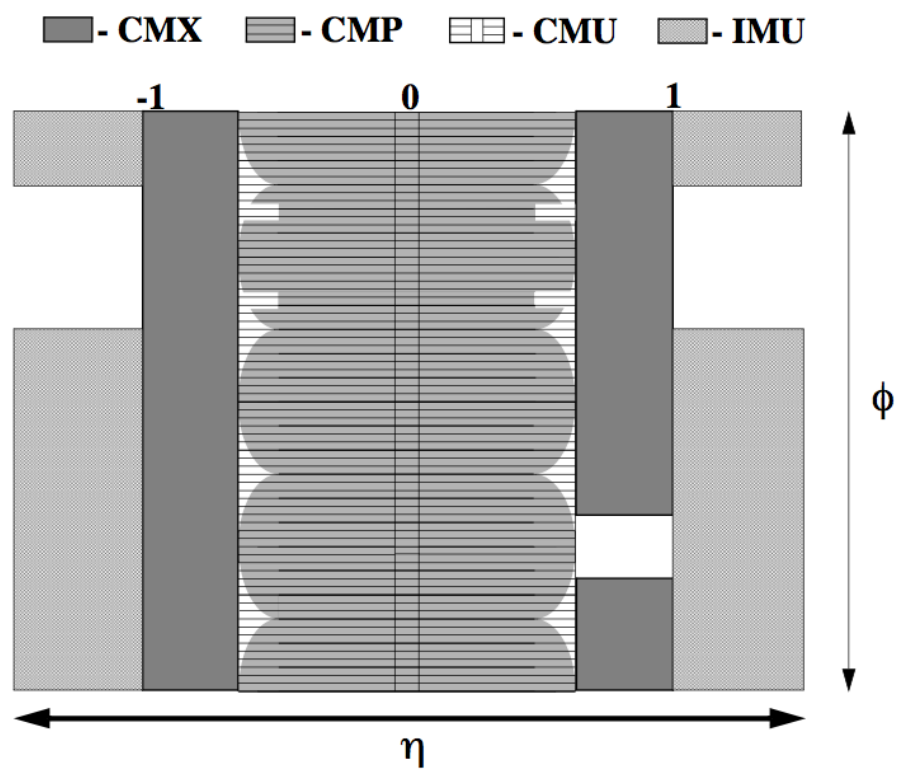

Figure 2.8 - Coverage of the muon detectors in the $\eta-\phi$ plane.

Muons do not interact strongly and when traversing matter lose energy almost exclusively through ionization ${ }^{10}$. At $90^{\circ}$ the minimum muon energy needed to traverse the central calorimeter and reach the first muon chamber is $1.4 \mathrm{GeV}$.

The muon system is the outermost layer of the CDF II detector, consisting of four layers of drift cells and one layer of scintillation counters, and is used to reconstruct track segments (stubs) of minimum ionizing particles and to provide accurate timing. Stubs are matched

\footnotetext{
${ }^{10}$ Muons are over 200 times more massive than electrons, so bremsstrahlung radiation, inversely proportional to the mass squared of the incident particle, is suppressed by a factor of $4 \times 10^{4}$ with respect to electrons.
}

Table 2.3 - Design parameters of the muon detectors. See [53, 54].

\begin{tabular}{l|cccc}
\hline \hline Parameter & CMU & CMP & CMX & IMU \\
\hline Pseudorapidity range & $|\eta|<0.6$ & $|\eta|<0.6$ & $0.6<|\eta|<1.0$ & $1.0<|\eta|<1.5$ \\
Azimuthal coverage [ $\left.{ }^{\circ}\right]$ & 360 & 360 & 360 & 270 \\
Maximum drift time [ns] & 800 & 1400 & 1400 & 800 \\
Drift tube cross section [cm] & $2.68 \times 6.35$ & $2.5 \times 15$ & $2.5 \times 15$ & $2.5 \times 8.4$ \\
Upstream interaction lengths & 5.5 & 7.8 & 6.2 & $6.2-20.0$ \\
Minimum $p_{T}(\mu)[\mathrm{GeV} / \mathrm{c}]$ & 1.4 & 2.2 & 1.4 & $1.4-2.0$ \\
\hline \hline
\end{tabular}


with the combined COT and SVX tracks, which provide momentum, direction, and vertex information. Four different systems $[53,54]$ detect muons in the $|\eta| \lesssim 1.5$ pseudo rapidity range. All types of muon detectors use single wire, rectangular drift chambers, arranged in arrays with fine azimuthal segmentation and coupled to scintillator counters. The scintillators are used for the triggers and for vetoes, while the drift chambers measure the $\phi$ coordinate given the drift time, and the $z$ coordinate using charge division. The chambers use a 50:50 admixture of argon and ethane, and operate in the proportional regime. The coverage of the four muon systems are depicted in Figure 2.8:

CMU: the CMU detector is located beyond the central hadronic calorimeter at a radius of $347 \mathrm{~cm}$ from the beam-line with coverage $0.03 \lesssim|\eta| \lesssim 0.63$. It is segmented into 24 azimuthal wedges of $15^{\circ}$. However, because of edge effects, only $12.6^{\circ}$ of each wedge is active, resulting in an overall azimuthal acceptance of $84 \%$. Each wedge is further segmented into three $4.2^{\circ}$ modules, each containing four radial layers of drift cells.

CMP: the CMP is a second set of muon drift chambers beyond the CMU and an additional $60 \mathrm{~cm}$-thick steel absorber. This material further reduces the probability of hadronic punch-through to the CMP. Muons need a transverse momentum of about $2.2 \mathrm{GeV}$ to reach the CMP. The CMP system is arranged in a box shape with similar acceptance to the $\mathrm{CMU}$ and serves as a confirmation of CMU tracks for higher momentum muons. A layer of scintillation counters (CSP) is mounted on the outer surfaces of the CMP chambers. The CMP and CMU have a large overlap in coverage and are often used together in identifying a muon track. The CMP helps to cover CMU $\phi$ gaps and the $\mathrm{CMU}$ covers the CMP $\eta$ gaps. Muon candidates which have both CMU and CMP stubs are the least contaminated by misidentified muons.

CMX: the CMX consists of drift tubes and scintillation counters (CSX) all arranged in a truncated conical shape. The CMX extends the muon pseudo-rapidity coverage to $0.6 \lesssim|\eta| \lesssim 1$ with 8 radial layers of drift chambers, each with a small stereo angle.

IMU: the IMU extends the pseudo-rapidity coverage even further to $1.0 \lesssim|\eta| \lesssim 1.5$. The IMU is mounted on the toroid magnets, which are not powered and just provide shielding.

Table 2.3 summarizes a few relevant design parameters of the detectors.

\subsection{CLC Detector}

The collider instantaneous luminosity as computed from measured beam parameters has an uncertainty of the order of $15-20 \%$. For this reason in CDF the collider luminosity is 
determined from the observed interaction rate in a detector whose cross section can be reliably computed. The measurement is made by gas Cherenkov counters (CLC) located in the pseudorapidity region $3.7<|\eta|<4.7$, which measure the average number of inelastic interactions per bunch crossing.

Each module consists of 48 thin, gas-filled, Cherenkov counters. The counters are arranged around the beam pipe in three conical layers, with 16 counters each pointing to the centre of the interaction region. The cones in the two outer layers are about $180 \mathrm{~cm}$ long, while the inner layer counters, closer to the beam pipe, have a length of $110 \mathrm{~cm}$. This geometry allows detection of particles produced at the collision point. The total signal of the counters allows separation of forward inelastic interactions from background and derivation of the collider luminosity from their observed rate.

\subsection{The CDF Trigger System}

$p \bar{p}$ collisions at Tevatron occur with a frequency of $2.5 \mathrm{MHz}$ (i.e every $396 \mathrm{~ns}$ ). The bunchbunch luminosity and the interaction cross-section are such that on average one or a few interactions take place in each bunch crossing. With an average event size of $\sim 250 \mathrm{~kb}$, this represents a huge amount of data which would flow through the CDF data acquisition system (DAQ). The CDF DAQ can sustain only a small fraction of this data flow: since the maximum rate for storing data to disk is $\sim 75 \mathrm{~Hz}$.

The trigger is the system that performs a quick online selection and keeps only events interesting for physics. A rejection factor of 10,000 is needed to match the DAQ capabilities. As shown in Figure 2.9, the CDF trigger is implemented in three levels of successively tighter and more sophisticated event selection. The first level is hardware-based; the second is a mixture of hardware and software, and the third is purely software-based, implemented in an on-line computer cluster.

\subsubsection{Level 1}

At Level 1 the decision logic is implemented in hardware: the selection algorithms are hard-coded into the electronic circuits of the trigger boards. In a synchronous pipeline up to 14 consecutive events can be stored for $\sim 5.5 \mu$ s while the hardware is making a decision. If no acceptance decision is made within that time the event is lost. Level 1 decisions are made on average in about $4 \mu \mathrm{s}$, so no dead time is expected from this trigger level. Level 1 rejects $97 \%$ of the events, reducing the input rate from $2.53 \mathrm{MHz}$ to an output rate of less than $40 \mathrm{kHz}$. The Level 1 decision is generated using information from:

- The XFT, which reconstructs transverse track parameters at low precision for $p_{T}>$ 


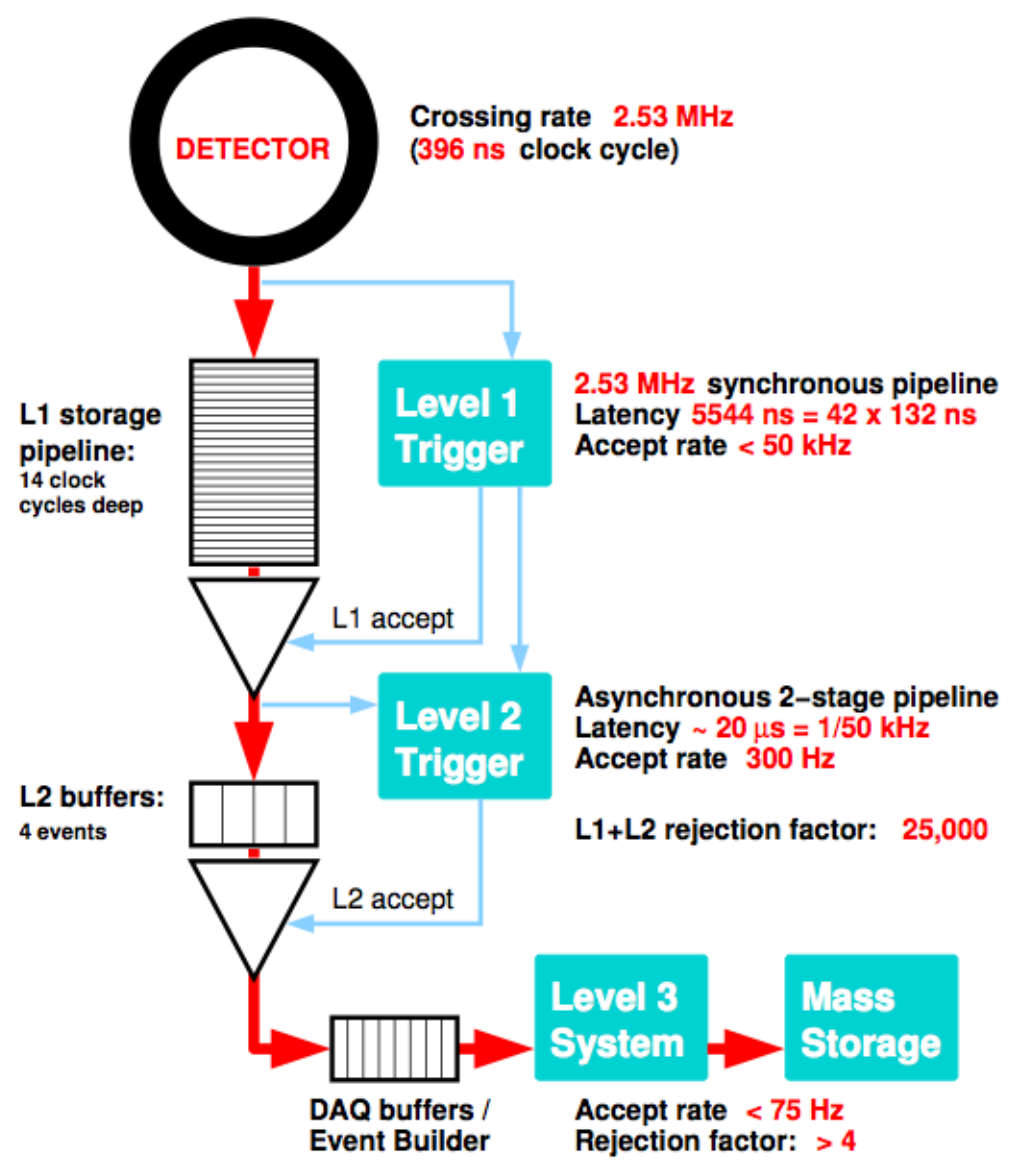

Figure 2.9 - Functional block diagram of the CDF II trigger and data acquisition systems.

$1.5 \mathrm{GeV} / \mathrm{c}$ by exploiting information from COT superlayers. These tracks are extrapolated to the calorimeters and muon chambers to contribute to all trigger levels;

- the calorimeter towers, which carry information on the electromagnetic and hadronic energy deposits (seeds, which can initiate electron, photon or jet identification);

- the muon stubs (segment of tracks reconstructed in the muon chambers), which are matched to the XFT tracks.

The XFT is a custom processor that can reconstruct tracks with an efficiency of about $95 \%$ and a fake rate of a few percent. The XFT has an angular segmentation of $1.25^{\circ}$, and an angular resolution of $0.3^{\circ}$. The momentum resolution is $\sigma_{p_{T}} / p_{T}^{2} \approx 0.017(\mathrm{GeV} / c)^{-1}[55]$. The XFT sends the tracks to an extrapolation unit (XTRP) feeding three Level 1 elements: L1CAL, L1TRACK, and L1MUON. L1CAL and L1MUON use extrapolated tracks and information from the calorimetry and muon systems to search for electrons, photons, jets, and muons. A decision stage uses the information from these low-resolution physics objects, called primitives. 


\subsubsection{Level 2}

Level 2 is an asynchronous system which processes events that have received a Level 1 accept in a FIFO (first in, first out) manner. It is structured as a two-stage pipeline with data buffering at the input of each stage. The first stage is based on dedicated hardware processors which assemble information from a particular section of the detector. The second stage consists of a computer which uses the list of objects generated by the first stage and implements in software the event selection. Each of the Level 2 stages is expected to take approximately $10 \mu \mathrm{s}$. The input buffers can store up to four events. After Level 2, the event rate is reduced to about $1 \mathrm{kHz}$ (rejection factor $\sim 40$ ). The reduction is achieved by:

- clustering the energy deposited in towers around Level 1 seeds, as an approximate measure of electron, photon or jet energy;

- using calorimeter and CES chamber information to improve separation of $e^{ \pm}$and $\gamma$ from hadrons;

- improving the matching between XFT tracks and muon stubs in order to have a better muon purity;

- and providing measurements of the track transverse impact parameters by means of the silicon vertex trigger (SVT), which allow the selection of events with secondary vertices indicating the presence of long-lived heavy-flavour hadron decay(s).

The SVT uses SVX $r-\phi$ hits to extend XFT track primitives into the SVX volume, closer to beam-line. The SVT improves the XFT $\phi_{0}$ and $p_{T}$ resolutions and allows a measurement of the impact parameter $d_{0}$ (the original XFT track primitives are assumed to come from the beamline).

\subsubsection{Level 3}

Level 3 is a software trigger. It is operated on a cluster of $\sim 300$ processors which reconstruct the entire event. The final decision to accept an event is made on the basis of a list of observables identifying candidate events of physical interest. Events that satisfy the Level 3 trigger requirements are passed on to the consumer server data logger system (CLS) for storage, first on disk and later on tape. The average processing time per event in Level 3 is on the order of $1 \mathrm{~s}$. 


\section{Chapter 3}

\section{Reconstruction of Physics Objects}

Particles generated in $p \bar{p}$ collisions are identified using the information provided by the CDF sub-detectors described in the previous chapter. From the raw data, high-level objects (such as tracks, vertices, and calorimeter clusters) are reconstructed and combined to identify physical objects, such as jets originating from $b$-quarks and missing transverse energy, both of which are of special interest for the analysis.

\subsection{Jets}

A jet is defined as a shower of particles produced in the hadronization of the originating parton (quark or gluon). Due to colour-charge confinement, quarks and gluons hadronize before they can be directly detected. A jet is mostly composed of hadrons like pions, kaons, protons and neutrons, as well as of electrons and muons. Figure 3.1 gives an illustration of the formation and the structure of a jet.

\subsubsection{Jet Clustering}

The energy of a jet can be calculated from the energy deposited in the calorimeter towers using a clustering algorithm. In this thesis jets are clustered using a cone algorithm named JETCLU [56] with a fixed cone size.

The JETCLU algorithm clusters together calorimeter towers with $E_{T_{i}}>1 \mathrm{GeV}$, where $E_{T_{i}}=$ $E_{i} \sin \theta_{i}$ is the transverse energy in a tower, and $E_{i}$ is the sum of the energies measured in the electromagnetic and hadronic compartments of that tower.

As a first step, seed towers are identified, ordered in decreasing $E_{T_{i}}$. Towers within a radius

$$
\Delta R \equiv \sqrt{\left(\eta^{\text {tower }}-\eta^{\text {jet }}\right)^{2}+\left(\phi^{\text {tower }}-\phi^{j e t}\right)^{2}}<0.4
$$




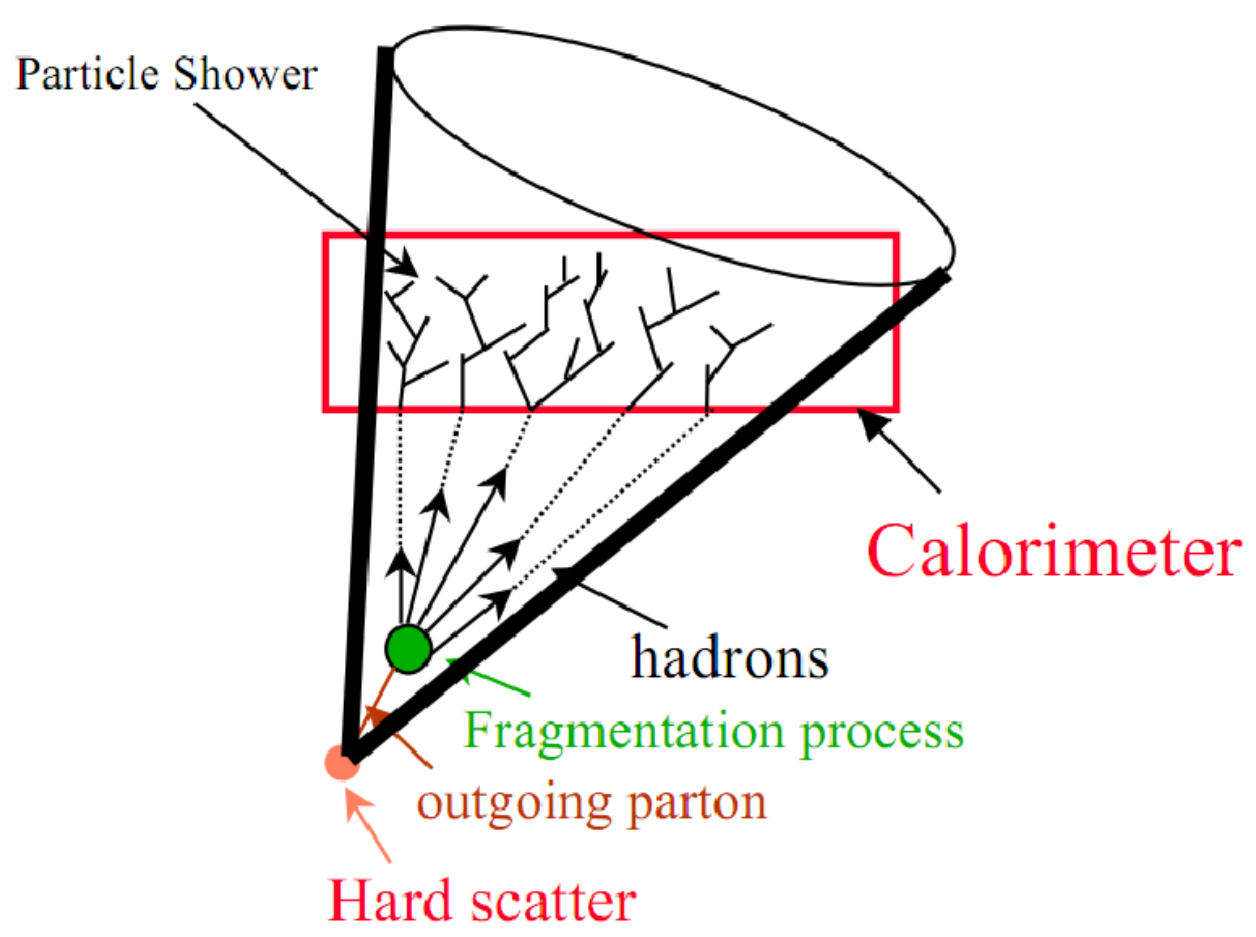

Figure 3.1 - Sketch of a jet shower with fragmentation and hadronization.

of a seed tower are combined into a cluster. Once we have an initial list of clusters, the jet transverse energy and the location (center) of the jet are calculated as follows:

$$
\begin{aligned}
E_{T}^{j e t} & =\sum_{i=0}^{N_{\text {tower }}} E_{T i} \\
\phi^{j e t} & =\sum_{i=0}^{N_{\text {tower }}} \frac{E_{T i} \phi_{i}}{E_{T}^{j e t}}, \\
\eta^{j e t} & =\sum_{i=0}^{N_{\text {tower }}} \frac{E_{T i} \eta_{i}}{E_{T}^{j e t}}
\end{aligned}
$$

where $N_{\text {tower }}$ is the number of towers inside the radius $\Delta R$ with $E_{T}>1 \mathrm{GeV}$. This procedure is repeated iteratively and a new list of towers around the new center is determined. The jet $E_{T}$ and direction are recalculated until the list of cluster towers and the cluster centroid are stable. Overlapping jets are merged into one jet if the overlap is more than $50 \%$ in the $\eta-\phi$ plane. When the overlap is smaller than $50 \%$, each tower in the overlap region is assigned to the nearest jet. The jet energy, momentum and coordinates, that form the raw jet information, are computed from the final list of towers. 


\subsubsection{Jet Energy Corrections}

The energy deposited in the calorimeter towers is only a part of the energy of the parton that produces the jet and needs to be corrected for detector effects and reconstruction algorithm imperfections.

At CDF the jet energy corrections are divided into different levels in order to correct independently for each bias that can distort the measured jet energy, such as response of the calorimeter to different particles, non-linear response of the calorimeter to the deposited energy, uninstrumented regions of the detector, energy released by final state particles not associated to the hard interaction of interest (spectators and multiple interactions), and jet energy radiated outside the integration cone. Depending on the physics analysis, a subset of these corrections can be applied.

The original parton transverse energy can be estimated by correcting the jet transverse energy for instrumental, radiation and fragmentation effects:

$$
p_{T}^{\text {parton }}=\left(p_{T}^{\text {jet }} \times C_{\eta}-C_{M I}\right) \times C_{A b s}-C_{U E}+C_{O O C}=p_{T}^{\text {particle }}-C_{U E}+C_{O O C},
$$

where:

- $p_{T}^{\text {parton }}$ : transverse momentum of the jet parent parton;

- $p_{T}^{\text {jet }}$ : jet transverse momentum;

- $p_{T}^{\text {particle }}$ : transverse momentum of the jet corrected by all instrumental effects and corresponds to the sum of the momenta of the hadrons, leptons, and photons within the jet cone;

- $C_{\eta}:$ : $\eta$-dependence" correction, ensuring homogeneous response over the entire angular range;

- $C_{M I}$ : "Multiple Interaction" correction, the energy to subtract from the jet due to pile-up of multiple $p \bar{p}$ interactions in the same bunch crossing;

- $C_{A b s}:$ "Absolute" correction of the calorimeter response to the momentum of the jet particles.

- $C_{U E}$ and $C_{O O C}$ : Underlying-event and out-of-cone corrections for radiation entering the jet cone but not belonging to the jet and for jet particles outside the jet cone, respectively. Note that these corrections are independent of the experimental setup, i.e., the CDF detector environment.

Details of the jet energy corrections for each of the effects presented above are described in Ref. [56]. This thesis uses "Level 5" (L5) corrections, which include the $\eta$-dependence correction, multiple-interaction correction, and the absolute correction. Corrections for underlying event and out-of-cone effects are not applied. 


\subsubsection{The H1 Algorithm}

The generic jet energy corrections shown in the previous section rely only on calorimeter information. For the analyses presented in this thesis jet energy corrections that employ tracking information are also applied.

Hadrons like $\pi^{ \pm}, \pi^{0}, K^{ \pm}, K_{S}, K_{L}, p$ and $n$ are produced by the parton initiating a jet. The momentum of a $\pi^{0}$ meson decaying to a pair of photons is measured by the EM calorimeter. The momentum of $\pi^{ \pm}, K^{ \pm}$, and $K_{S}$ decaying to $\pi^{+} \pi^{-}$can be measured in either the tracking system or the calorimeter system. Only $K_{L}$ mesons and neutrons are measured solely by the hadron calorimeter.

The majority of the charged hadrons that form a jet have low momenta and the tracking detectors are able to measure their energy to a much higher precision than the calorimeter. The estimate of the jet energy can be improved using the tracking information. We apply an algorithm based on a method developed by the H1 collaboration to correct the jet energy using tracker information [57]. This algorithm is applied subsequent to the generic jet energy corrections.

In order to have a reliable momentum measurement, tracks used in the $\mathrm{H} 1$ algorithm must satisfy the following requirements:

- $0.5<p_{T}^{\text {track }}<15 \mathrm{GeV} / c$,

- $N_{\text {COT }}>25$ and $\left|\eta_{\text {track }}\right|<0.8$

- $z_{0}<60 \mathrm{~cm}$

where $p_{T}^{\text {track }}$ is the transverse momentum of the track, $N_{\text {COT }}$ is the number of COT hit associated to the track, $\eta_{\text {track }}$ is the pseudo-rapidity of the track and $z_{0}$ is the distance of the closest approach to the beam line.

The tracks that satisfy the above conditions are sorted by ascending $p_{T}^{\text {track }}$ and extrapolated to the surface of the calorimeter. Towers with $\left|\eta_{\text {tower }}-\eta_{\text {track }}\right|<0.1$ and $\mid \phi_{\text {tower }}-$ $\phi_{\text {track }} \mid<0.2$ are considered as containing the full energy of the track. These conditions are based on a study of simulated single pion events.

The selected towers are sorted in order of distance to the track. If the track energy is greater than the total energy of the selected towers, the energy in the towers is replaced with the track energy and the towers are removed from further consideration ("locked"). If this is not true, the energy in the first $n$ towers are locked. The $(n+1)^{\text {th }}$ tower is also locked and its energy is scaled such that the total locked energy is equal to the track energy. The process is repeated for each track. Towers already locked by a previous track are not considered for subsequent tracks. The total energy of the jet is the scalar sum of the $p_{T}$ of all selected tracks and all non-locked tower energies. 
It is possible for a single tower to collect the energy of many particles, including neutral particles. In these cases the calorimeter tower energy exceeds the track energy. For these high density regions the $\mathrm{H} 1$ algorithm will not correct the calorimeter energy and the energy measurement will not be improved. Since a jet usually contains regions of both high and low densities, the $\mathrm{H} 1$ algorithm provides modest improvements in the jet energy resolution compared to the jet energy measurements performed using only the calorimeter information. However, even small improvements in energy resolution are crucial. In searches for small signals, like searches for a low mass $H \rightarrow b \bar{b}$ [60], the use of the H1 algorithm substantially improves the sensitivity of the search, increasing the acceptance to signal events by $\approx 10 \%$. The $\mathrm{H} 1$ algorithm also helps recover events where part of the energy is lost because energetic charged particles hit un-instrumented regions of the calorimeter. By properly reconstructing their energy, the $\mathrm{H} 1$ algorithm retains events that would have otherwise been rejected by the candidate event selection cuts.

\section{$3.2 b$-Jets}

Jets originating from bottom quarks are present in many interesting processes. The top quark nearly always decays to a $b$-quark and a $W$ boson; a $125 \mathrm{GeV} / c^{2} \mathrm{SM}$ Higgs boson is expected to decay to a $b$-quark pair more than $60 \%$ of the time. "Tagging" $b$-quark jets helps to identify these processes by reducing the light-flavour-quark background.

Bottom-flavoured hadrons have sufficient lifetime that they can travel a measurable distance before decaying. A $B^{+}$meson has $c \tau=492 \mu \mathrm{m}$; with the typical production momentum in a Tevatron event, it travels a few millimeters before decaying, which is enough to be identified by the tracking detectors. In the case of the single-top s-channel, the mean transverse momentum of a $B$-hadron in the event is $\approx 50 \mathrm{GeV} / c$. Since $B$-hadrons have a mass of $\approx 5 \mathrm{GeV} / c^{2}$, the corresponding boost is $\beta \gamma \approx 10$. Accordingly, the impact parameter of each secondary-vertex track is $\approx 5 \mathrm{~mm}$.

$B$-hadron decays produce sub-jets composed of tracks with large impact parameter, hence with low probability of coming from the primary vertex. The impact parameter $d_{0}$ is reconstructed in the silicon detector with a precision of $\approx 25 \mu \mathrm{m}$, making it possible to separate displaced tracks from prompt tracks coming from the primary interaction, as shown in Figure 3.2.

A bottom hadron in a $b$-quark-initiated jet has a larger mass than other hadrons in the jet, and is much more massive than its decay particles. As a consequence, in a $b$-jet the $b$-hadron decay particles dominate the phase space of large transverse momentum to the jet axis. This causes the $b$-jet to be wider, and to have a higher particle multiplicity and invariant mass. The $\approx 10 \%$ branching ratio of $B$-hadrons to leptons can also be used to identify $b$-jets. 


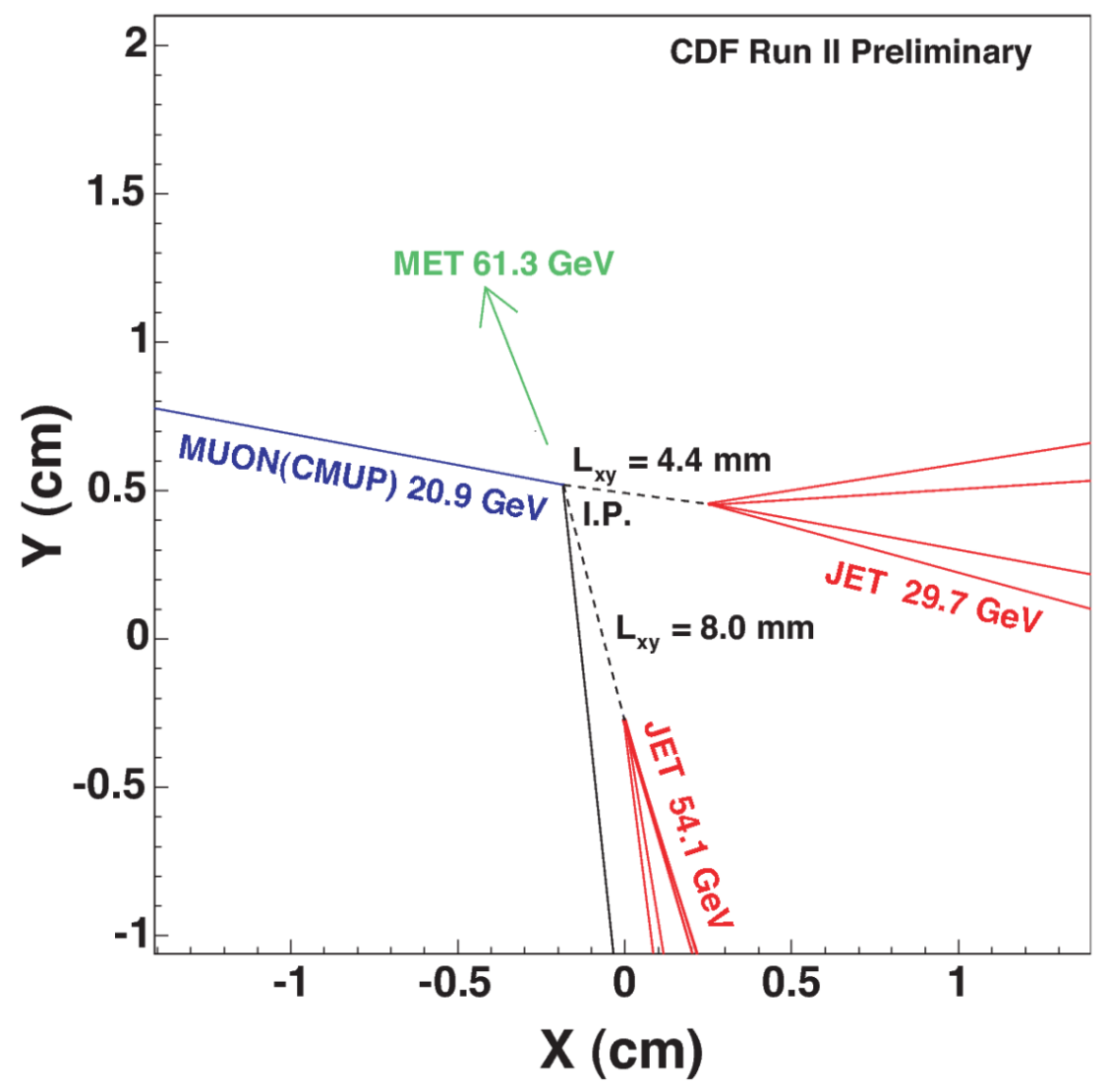

Figure 3.2 - Candidate event of $W+$ jets production (run 166063, event 279746) with two secondary vertices tagged. The $\vec{E}_{T}$ direction, a muon track, a prompt track and tracks from the secondary vertices are shown

These unique features can be used in "b-tagging" algorithms to identify $b$-jets. In this thesis three different $b$-tagging algorithms are used: SECVTX, JETPROB, and HOBIT ${ }^{1}$.

\subsubsection{The SECVTX Algorithm}

The SECVTX algorithm [61] relies on the displacement of secondary vertices relative to the primary event vertex to identify jets originating from $b$-quarks. The algorithm searches for secondary vertices on a per-jet basis, using the tracks within the jet cone and with large $d_{0}$ with respect to the primary vertex, requiring $\left|d_{0}\right|>1.5 \mathrm{~mm}$.

A precise knowledge of the collision point is necessary in order to select displaced tracks coming from decays of $B$-hadrons. If there is an identified high-momentum electron or muon in the event, the primary vertex is the nearest vertex to these objects. For events without high-momentum leptons, the algorithm uses the vertex which has the highest total scalar sum of transverse momentum of associated tracks. The position of the primary

\footnotetext{
${ }^{1}$ No specific techniques are used to identify $c$-jets in this thesis. The signal processes show only $b$-jets in the final state, while the discrimination from the background processes with $c$-jets is performed indirectly exploiting different kinematic properties.
} 
vertex is then determined by fitting a set of tracks within a $\pm 1 \mathrm{~cm}$ window in $z$ around this vertex and with impact parameter significance (relative to the beam line) $d_{0} / \sigma_{d_{0}}<3$, where $\sigma_{d_{0}}$ includes the uncertainties on both the track and the beam line positions.

Secondary vertex tagging operates on tracks that satisfy requirements on the transverse momentum, the number of silicon hits attached to the tracks, the quality of those hits, and the $\chi^{2}$ /ndf of the final track fit. Jets are defined as "taggable" if they have two good quality tracks.

Once a secondary vertex is found in a jet, the two-dimensional decay length $L_{2 D}$ is calculated as the projection onto the jet axis of the vector pointing from the primary vertex to the secondary vertex in the $r-\phi$ view. When the absolute difference $|\Delta \phi|$ between the jet axis and the secondary vertex vector is less than $90^{\circ}$ the sign of $L_{2 D}$ is positive, so that large positive $L_{2 D}$ are expected for secondary vertices corresponding to the decay of $B$ - and $D$-hadrons. For light-flavour hadrons, the $L_{2 D}$ distribution peaks at zero and the width of the distribution is determined by the tracking resolution. To identify a good secondary vertex, the SECVTX algorithm requires $L_{2 D} / \sigma_{L_{2 D}}>7.5$, where $\sigma_{L_{2 D}}$ is the total estimated uncertainty on $L_{2 D}$ including the error on the primary vertex. At most one good secondary vertex per jet can be found by the algorithm and a jet is defined as tagged if it contains a good secondary vertex.

To calculate the contribution from mistags, defined as the background from the false secondary vertices, the negative tags with a vertex $L_{2 D} / \sigma_{L_{2 D}}>-7.5$ are used. The mistags will be further discussed in Section 4.3.2.

To accommodate the needs of different analyses, loose, tight, and ultratight SECVTX tags are defined by varying the requirements on the track selection and the secondary vertex quality. For example, in top-quark-pair analyses where the distinctive kinematic properties of a $t \bar{t}$ event are used to keep the background contamination low, a loose selection of $b$-jets allows for a larger signal acceptance, while the tight SECVTX selection provides the optimal purity for the Higgs boson search.

\subsubsection{The JETPROB Algorithm}

The JETPROB (jet probability $b$-tagging) algorithm [62] is used along with SECVTX in order to increase the efficiency for identifying $b$-jets. It uses tracks associated to a jet to determine the probability for the ensemble of tracks to be consistent with originating from a primary vertex (primary jet). The calculation of the probability is based on the impact parameters $d_{0}$ of the tracks in the jet and their uncertainties.

The tracks in a jet which originate from a light parton should point to the primary vertex, but some of these tracks are reconstructed with a nonzero impact parameter due to the finite tracking resolution. They have an equal probability of being positively or nega- 

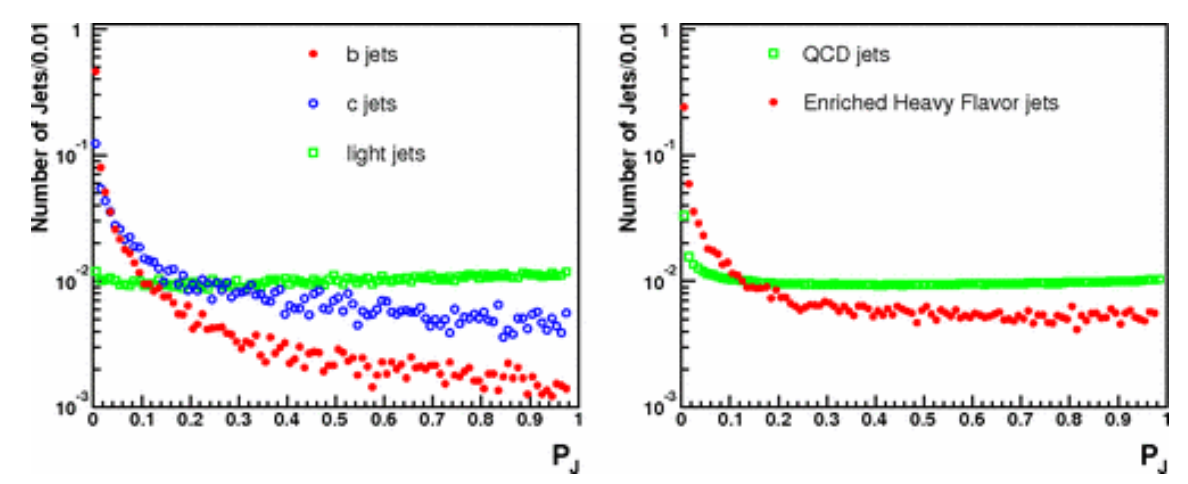

Figure 3.3 - Left: JETPROB distributions for jets matched to $b$ (full circles), $c$ (empty circles), and light (empty squares) quarks in MC simulated events. Right: JETPROB distributions for electron jets in inclusive electron data (full circles) and for generic QCD jets in Jet50 data (empty squares). From [62].

tively signed. The probability for tracks originating from the primary vertex is made by construction uniform from 0 to 1 .

Tracks produced by the decay of a high-momentum heavy-flavour quark are usually displaced in the jet direction and preferentially populate the positive side of the signed impact parameter distribution. The width of the negative impact parameter distribution is primarily due to the tracking detector resolution, beam spot size, and multiple scattering. A jet containing a heavy-flavour hadron producing displaced tracks has a low probability to originate fully from a primary vertex, so its JETPROB distribution is sharply peaked at 0 (Figure 3.3).

In the JETPROB algorithm, the tracks in a jet are divided into 72 different categories according to the number and quality of SVX hits, detector $\eta$, and $p_{T}$. The signed impact parameter significance $S_{d_{0}} \equiv d_{0} / \sigma_{d_{0}}$ is parametrized for each track category. An example of the $S_{d_{0}}$ distribution is shown in Figure 3.4.

Assuming tracks have negative impact parameter due to detector resolution, the probability $P_{J}$ for a jet to be consistent with a zero lifetime hypothesis is:

$$
P_{J}=\left[P i_{i}=1_{t}^{N} r k P_{t} r k\right] \times\left[\begin{array}{l}
N t r k-1 \\
k=0
\end{array}\left(\ln \Pi_{i}=1_{t}^{N} r k P_{t} r k\right)^{k} / k !\right],
$$

where $N_{t r k}$ is the number of tracks with positive impact parameter and:

$$
P_{t r}\left(S_{d_{0}}\right)=\frac{\int_{-\infty}^{-\left|S_{d_{0}}\right|} R(S) d S}{\int_{-\infty}^{0} R(S) d S},
$$

where $R(S)$ is the resolution function from the negative side of the $S_{d_{0}}$ distribution.

The JETPROB algorithm has a continuous output from 0 to 1 and a threshold is chosen depending on the desired $b$-tag efficiency and light-jet efficiency ("mistag rates"). In this thesis, jets with $P_{J}<0.05$ are defined as JETPROB-tagged $b$ jets. 


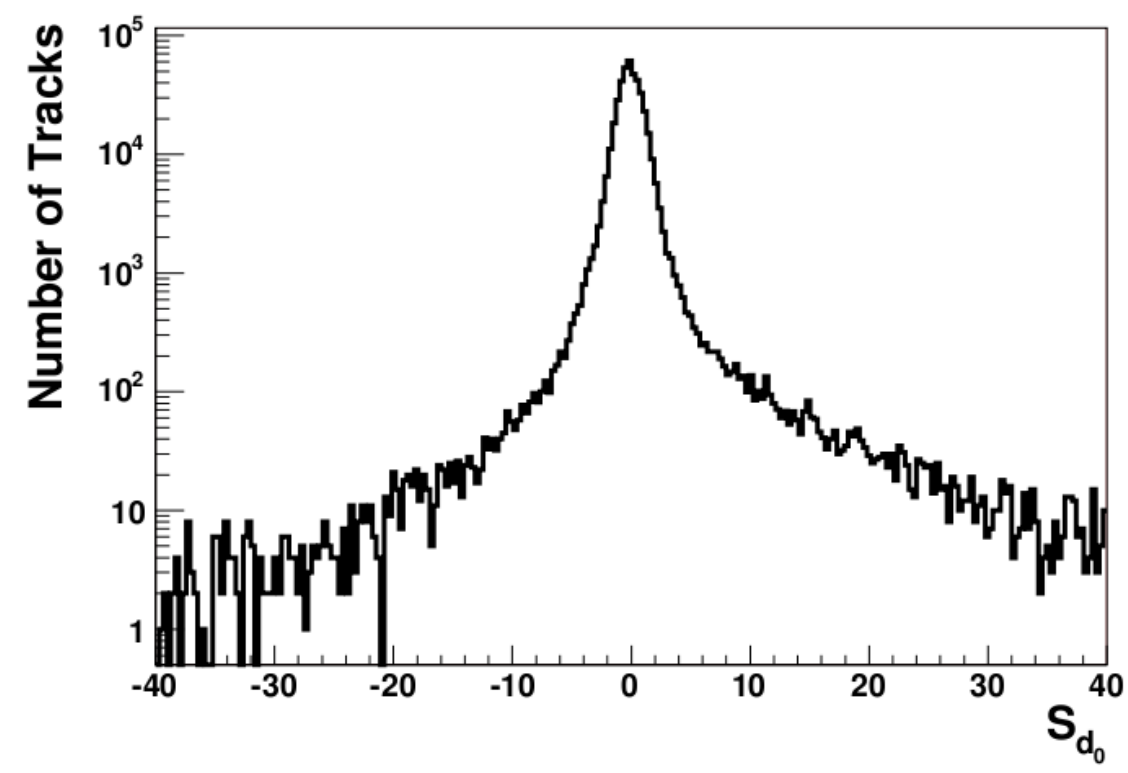

Figure 3.4 - Distribution of the impact parameter significance for tracks in an inclusive jet sample with at least 5 good SVX hits, $p_{T}>5 \mathrm{GeV} / \mathrm{c}$, and $|\eta|<0.6$. From [62].

\subsubsection{The HOBIT Algorithm}

The Higgs-optimized $b$-identification tagger (HOBIT) [63] is an algorithm optimized to identify $b$-jets produced by Higgs-boson decays. The HOBIT algorithm employs a multivariate technique to discriminate between light- and heavy-flavour jets. More details on multivariate techniques used in particle physics can be found in Ref. [64].

The HOBIT algorithm was trained on a set of 25 input variables, incorporating features of the other CDF $b$-taggers, such as the output of the CDF soft muon tagger, and the inputs to the RomaNN [65] and $b$-ness [66] multivariate taggers. The full list of variables is:

- Jet $E_{T}$ - transverse energy corrected to L5;

- RomaNN inputs:

- for the most significantly displaced vertex, the pseudo- $c \tau^{2}$, the 3-dimensional displacement and displacement significance, and the invariant mass of the tracks forming the vertex;

- the number of tracks in displaced vertices plus the number of standalone displaced tracks; the combined invariant mass of all displaced tracks; the ratio of the scalar sum of the $p_{T}$ of these tracks to the scalar sum of the $p_{T}$ of all tracks in the jet;

- the loose SECVTX output and the mass of the tracks used in the loose SECVTX vertex fit.

\footnotetext{
${ }^{2}$ pseudo-c $\tau=L_{x y} * \frac{M^{v t x}}{P_{T}^{v t x}}[65]$.
} 


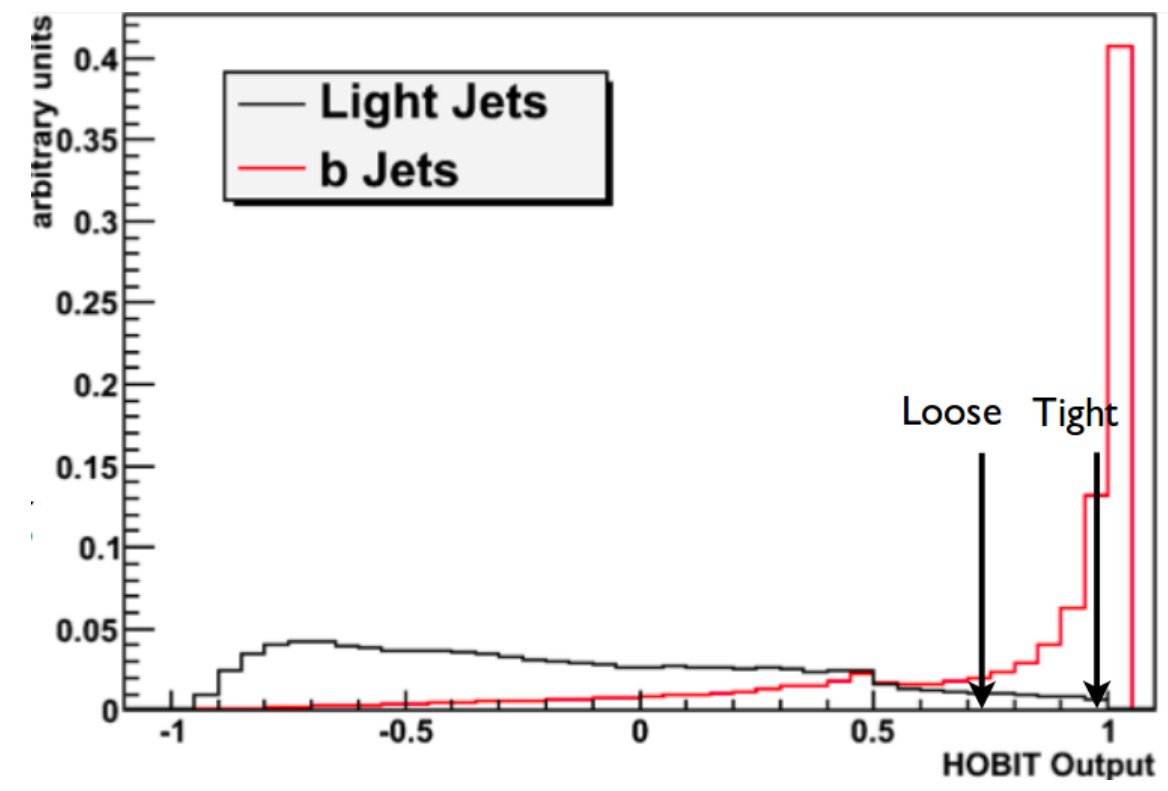

Figure 3.5 - The HOBIT output distribution. The black histogram is for light-flavour jets and the red histogram is for $b$-jets. Both distributions are MC simulations normalized to unity. From [63].

- $b$-ness inputs:

- the ten highest track-by-track neural-network output values for tracks in a jet ${ }^{3}$.

The neural network was trained using $W H\left(\mathrm{~m}_{H}=120 \mathrm{GeV} / \mathrm{c}^{2}\right)$ Monte Carlo events for the $b$-jet sample and $W+$ jets Monte Carlo events for the light-flavour-jet sample. The $E_{T}$ spectrum of light-flavour jets in $W+$ jets events is reweighted to match the $E_{T}$ spectrum of $b$-jets in $W H$ events, in order to ensure an $E_{T}$-independent output for the HOBIT algorithm.

In Figure 3.5 the HOBIT output, ranging from -1 to 1 , is shown. Jets are defined as loose HOBIT-tagged jets if the output is larger than 0.72 and as tight HOBIT-tagged jets if the output is larger than 0.98. In Figure 3.6, the $b$-jet efficiencies and the light-jet efficiencies as a function of jet $E_{T}$ and $\eta$ are shown for the two HOBIT operating points. Figure 3.7 shows a comparison between the $b$-jet efficiencies and the light-jet efficiencies for the HOBIT algorithm and for the other $b$-taggers in use at CDF.

\subsection{Missing Transverse Energy}

The momentum carried by non-detectable particles (like neutrinos) is denoted as missing energy. The energy of protons and antiprotons that collide at the Tevatron is split between

\footnotetext{
${ }^{3}$ The $b$-ness tagger uses the properties of individual tracks to determine whether a jet is $b$-like. To evaluate the information from individual tracks, the $b$-ness tagger utilizes a neural network (track-by-track NN). See Ref. [66]
} 

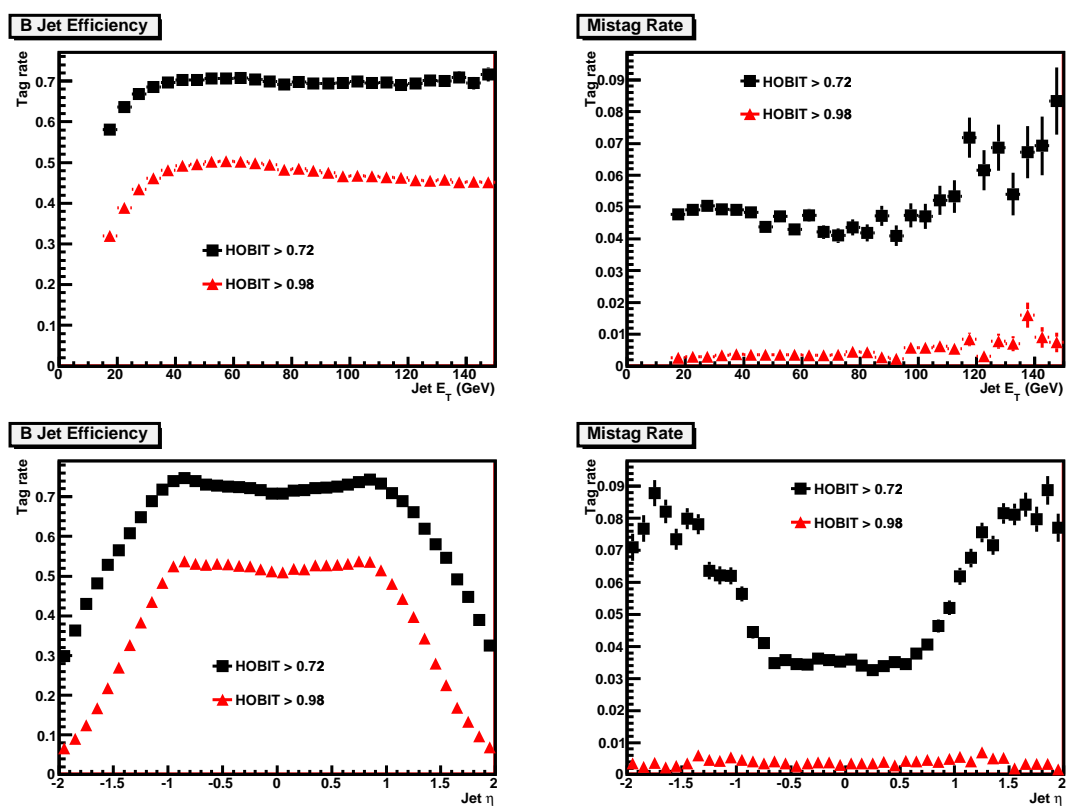

Figure 3.6 - The $b$-jet and light-jet efficiencies in MC before scale factor corrections as a function of $\eta$ and $E_{T}$. The black triangles are for the looser operating point and the coloured triangles are for the tighter operating point. From [63].

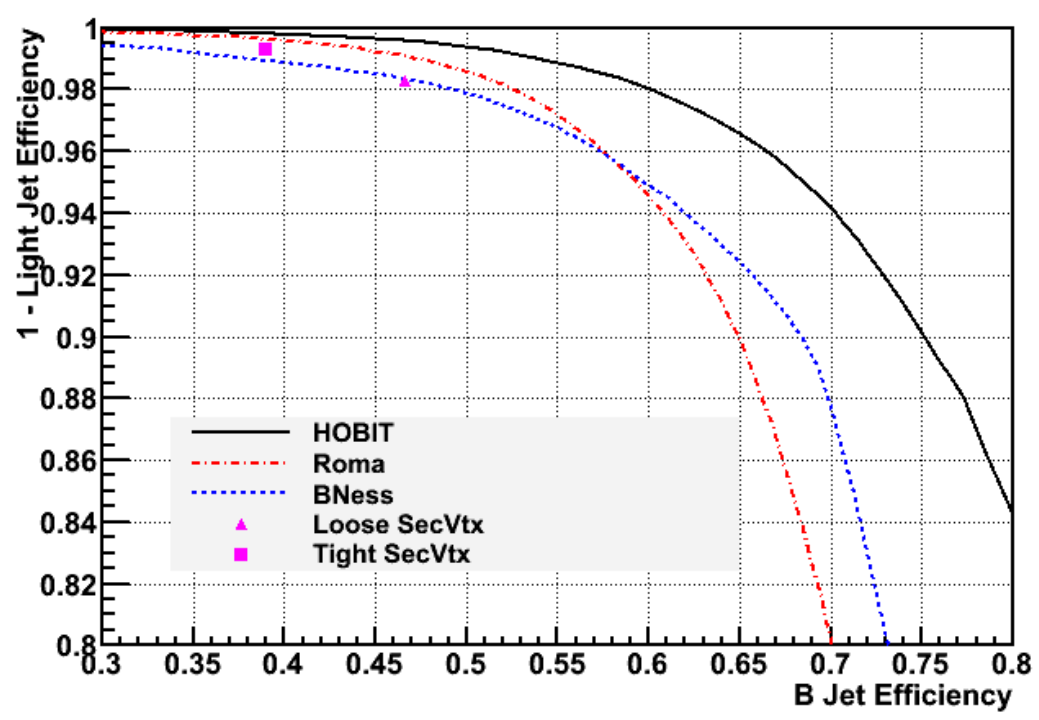

Figure 3.7 - The $b$-jet and light-jet efficiencies for the HOBIT tagger compared to the other CDF taggers. From [63]. 
their constituent quarks and gluons and is constantly exchanged, so that the initial momentum of the colliding partons along the beam axis is unknown and the total amount of missing energy cannot be determined in the $z$ direction. However, the colliding partons carry no transverse momentum on average so the missing energy in the transverse direction can be calculated using the conservation of momentum. The missing transverse energy $\left(\mathbb{E}_{T}\right)$ can be defined as the magnitude of the vector sum of the transverse particle momenta measured in all the calorimeter towers. Any transverse energy imbalance in the detector may indicate that a particle left the detector without interacting with its material. Many interesting physics processes contain significant $\mathbb{E}_{T}$, which is used to discriminate these processes from the background.

The analyses described in this thesis select events with large missing transverse energy in the final state; therefore an accurate reconstruction of this quantity is important. In the Level 3 trigger the raw $\mathbb{E}_{T}$ is calculated using the transverse energies measured in both the electromagnetic and hadronic calorimeters:

$$
\vec{E}_{T}=-\sum^{\text {towers }}\left(E_{i} \sin \theta_{i}\right) \hat{n}_{i},
$$

where $E_{i}$ is the energy of the $i$ th tower, $\theta_{i}$ is the polar angle of the line pointing from the center of the detector $(z=0)$ to the $i$ th tower, and $\hat{n}_{i}$ is a transverse unit vector pointing to the center of each tower. Offline the $\mathbb{E}_{T}$ is reconstructed using the primary vertex $z_{0}$ and $d_{0}$. The sum is taken over all towers that are above a threshold of $0.1 \mathrm{GeV}$ and extends to an $|\eta|$ of 3.6; the forward calorimeters are not used since uninstrumented regions designed to accommodate the quadrupole focusing magnets affect the measurement.

After the application of the jet energy corrections described in Sections 3.1.2 and 3.1.3, the $\mathbb{E}_{T}$ is recomputed using:

$$
\begin{aligned}
\mathbb{E}_{x}^{\text {corr }} & =\mathbb{E}_{x}^{\text {raw }}-\sum_{i=1}^{N_{\text {jets }}}\left(E_{x}^{\text {corr }, i}-E_{x}^{\text {raw }, i}\right) \\
E_{y}^{\text {corr }} & =\mathbb{E}_{y}^{\text {raw }}-\sum_{i=1}^{N_{\text {jets }}}\left(E_{y}^{\text {corr }, i}-E_{y}^{\text {raw }, i}\right) . \\
\phi_{E_{T}^{c o r r}} & =\tan ^{-1}\left(\frac{\mathbb{E}_{y}^{\text {corr }}}{\mathbb{E}_{x}^{\text {corr }}}\right)
\end{aligned}
$$

The $\mathbb{E}_{T}$ used in this thesis is the corrected $\mathbb{E}_{T}$. The $\mathbb{E}_{T}$ is further corrected for the presence of muons since, being minimum ionizing particles, they can pass through the calorimeter, without substantially losing energy.

While large $\mathbb{E}_{T}$ is expected in events that contain particles that do not interact, detector effects may also lead to a significant amount of $\mathbb{E}_{T}$ : 
- when protons or antiprotons of the Tevatron beam collide with nuclei of gas atoms or beam collimators, they produce a "halo" of muons, travelling roughly parallel to the beam. Some of these muons cross a row of calorimeter towers along the $z$-axis depositing energy in the calorimeters asymmetrically in $\phi$.

- problems with some of the calorimeter tower electronics or calibrations may cause the tower to report an inaccurate value of the energy of the incident particles.

- a cosmic muon travelling through the detector can produce $\mathbb{E}_{T}$ if the muon is not reconstructed on one side of the detector.

- when the direction of an energetic jet is near an uninstrumented calorimeter region, the energy of the jet will be underestimated.

For analyses requiring $\mathbb{E}_{T}$ and jets, the $\mathrm{CDF} \mathbb{E}_{T}$ working group recommends a set of selections to remove the instrumental sources of $\mathbb{E}_{T}$. The criteria are described in Ref. [67] and summarized as follows:

- Pass 1

- There must be at least one central jet with $\left|\eta_{\text {det }}\right|<0.9$ and $E_{T}>10 \mathrm{GeV}$.

- The event electromagnetic fraction (EEMF) must be larger than 0.1, where

$$
E E M F=\frac{\sum_{j=1}^{N_{j e t}} E_{T}^{j} \cdot E M F_{j}}{\sum_{j=1}^{N_{j e t}} E_{T}^{j}}
$$

and $E M F_{j}$ is the fraction of the jet energy deposited in the electromagnetic calorimeter. Only jets with $E_{T}>10 \mathrm{GeV}$ are considered in the EEMF calculation.

- There must be at least one COT track with $p_{T}>0.5 \mathrm{GeV} / c$ and one axial superlayer with six or more hits on it.

- Pass 2

- The event charged fraction (ECHF) must be larger than 0.1, where

$$
E C H F=\frac{\sum_{j=1}^{N_{j e t}} C H F_{j}}{N_{j e t}}
$$

and $\mathrm{CHF}_{j}$ is the jet charge fraction which is defined as the ratio of the sum $p_{T}$ of the jet tracks to the jet $E_{T}$.

- At least one good primary vertex in the event.

- Pass 3 
- Any jet with $E_{T}>10 \mathrm{GeV}$ that is in the "chimney" region of the calorimeter is excluded.

- The event primary vertex must have $|z|<60 \mathrm{~cm}$.

Events that fail any of the Pass 1-3 requirements are discarded from the analysis.

\subsection{Lepton Identification}

Lepton reconstruction depends on the type of lepton and its location inside the detector. Although this thesis focuses on events with energetic jets and large missing transverse energy, lepton identification is important since we reject events with identified leptons (electrons or muons), ensuring statistical independence with other CDF analyses probing the same physical processes. This facilitates the combination of the results. Rejected leptons are identified using the standard CDF selection criteria [68].

\subsubsection{Electron Identification}

Central electrons are those in the coverage of the central calorimeter and are identified with loose selection criteria based on information from the EM calorimeter and the COT. The selection requirements are:

- a COT track in the central EM calorimeter region $(|\eta|<1.1)$ and:

$-\left|z_{0}\right|<60 \mathrm{~cm}$

- $p_{T} \geq 10 \mathrm{GeV} / c$;

- 3 axial superlayers (SLs) with 5 hits/SL, and 2 stereo SLs with 5 hits/SL;

- $E_{T} \geq 20 \mathrm{GeV}$;

- not from the photon conversion;

- isolation $p_{T}(\Delta R=0.4) / E_{T}<0.1, p_{T}(\Delta R=0.4)$ represents the scalar sum of the $p_{T}$ of all other tracks in the cone radius $\Delta R \equiv \sqrt{(\Delta \eta)^{2}+(\Delta \phi)^{2}}=0.4$;

- very small hadronic calorimeter deposition, $E_{\text {had }} / E_{E M} \leq 0.055+0.00045 \times E$.

The plug electrons are identified with selection criteria based on information from the plug EM calorimeter (PEM) and the plug shower maximum detector (PES):

- $1.2 \leq|\eta| \leq 2.8$ 
- $E_{T} \geq 20 \mathrm{GeV}$;

- isolation $p_{T}(\Delta R=0.4) / E_{T}<0.1$;

- $E_{H a d} / E_{E m} \leq 0.05$;

- PEM $3 \times 3 \chi^{2} \leq 10$, where the $\chi^{2}$ is the result of a comparison of the PEM shower profile of the electron candidate with the measurements from test beam electrons. The test beam results for PEM were obtained using a $3 \times 3$ cluster size;

- PES $5 \times 9 \mathrm{U} \geq 0.65$, where $5 \times 9 \mathrm{U}$ is the $5 / 9$ profile ratio of the $\mathrm{u}$-strips of the best matching $2 \mathrm{~d}$ PES cluster of the PEM shower profile of the electron candidate that matches the measurements from test beam electrons;

- PES $5 \times 9 \mathrm{~V} \geq 0.65$, where $5 \times 9 \mathrm{~V}$ is the $5 / 9$ profile ratio of the $\mathrm{v}$-strips of the best matching $2 \mathrm{~d}$ PES cluster of the PEM shower profile of the electron candidate that matches the measurements from test beam electrons;

- $\triangle R$ between the PES and PEM centroids $\leq 3.0 \mathrm{~cm}$.

\subsubsection{Muon Identification}

The central muons are identified with a combination of selection criteria from the COT, muon chambers and calorimeter:

- $p_{T}>20 \mathrm{GeV} / c$;

- $\mathrm{EM}$ energy $<2 \mathrm{GeV}+\max (0 ; 0.0115 \times(p-100))$;

- Hadronic energy $<6 \mathrm{GeV}+\max (0 ; 0.028 \times(p-100))$;

- isolation $p_{T}(\Delta R=0.4) / p_{T} \leq 0.1$;

- with 3 axial SLs with 5 hits/SL, and 2 stereo SLs with 5 hits/SL;

- $\operatorname{COT}$ track $z_{0} \leq 60 \mathrm{~cm}$;

- $\left|d_{0}\right|<0.2 \mathrm{~cm}$ (track with no silicon hits);

- $\left|d_{0}\right|<0.02 \mathrm{~cm}$ (track with silicon hits);

- for CMU and CMP muons, $\left|\delta_{x}(C M U)\right|<7 \mathrm{~cm}$ and $\left|\delta_{x}(C M P)\right|<5 \mathrm{~cm}$, where $\delta_{x}$ is the difference between the muon stub position and the extrapolated track position;

- for CMX muons and run number $>150144,\left|\delta_{x}(C M X)\right|<6 \mathrm{~cm}$ and COT exit radius less than $140 \mathrm{~cm}$. 


\section{Chapter 4}

\section{Model of the Selected Data Sample}

\subsection{Data Sample}

Data collected by the CDF experiment is organized into "runs" [69] within each Tevatron store, which normally last about 16 hours. A run or a section of the run is marked as good if the detector was functional during that period. The "qcd si GR-LvHiggs" list of good runs, which includes runs recovered from all data periods, is used in the work presented in this thesis.

The full dataset collected by the CDF II detector in the Tevatron Run II is analyzed. The data were collected from March 2002 to the shutdown of the Tevatron collider on September 30, 2011. The luminosities are measured by the Cherenkov Luminosity Counter (CLC) period by period. The corrected luminosity is $1.019 \times$ CLC luminosity [70]. The full dataset corresponds to an integrated luminosity of $9.45 \mathrm{fb}^{-1}$.

\subsection{Event Selection}

\subsubsection{Online Requirements and Trigger Efficiency}

This thesis work is based on the analysis of events with a large imbalance in the transverse energy. Online, the most efficient triggers for collecting events with large $\mathbb{E}_{T}$ are chosen. Data from the MET_DIJET, MET35_CJET, and MET40/45 triggers are analyzed. These $\mathbb{E}_{T^{-}}$ based triggers select events using detector information at each of the three levels of the CDF trigger system.

At Level 1 the calorimeter towers are organized into a $24 \times 24$ array of trigger towers in the $\eta-\phi$ plane. Using a primary vertex of $z=0$, the transverse projection of the tower energies is computed and the missing energy is calculated as the vector sum of the trigger towers. The missing energy at Level 1 has poor resolution due to the limited information 
that can be collected in the $5.5 \mu$ s decision time, and it is usually underestimated. In order to maximize efficiency, the $\mathbb{E}_{T}$ threshold at Level 1 is chosen to be as low as possible. The selection applied at Level 1 is:

- JET10: at least one central jet $(|\eta|<1.1)$ or plug jet $(1.1<|\eta|<3.6)$ with $E_{T}>10$ $\mathrm{GeV}$;

- MET28: $\mathbb{E}_{T}>28 \mathrm{GeV}$.

Level 2 uses a simple algorithm developed during Run I to reconstruct jets as "clusters" of energy deposits. The algorithm finds a "seed" tower with an energy of at least $3 \mathrm{GeV}$, and then clusters adjacent towers imposing an energy threshold of $1 \mathrm{GeV}$. The process is iterated until no more towers that satisfy the minimum energy requirement are found. The seed tower coordinates define the cluster position. For the low instantaneous luminosities present in the initial phase of Run II the algorithm is efficient, but its application can cause mismeasurement as the luminosity increases. In high luminosity events, multiple interactions significantly affect the detector occupancy and lead to the presence of many spurious energetic towers around the seed tower. As a consequence, the algorithm can erroneously add those towers to the seed. The $\mathbb{E}_{T}$-based triggers require the presence of at least two clusters in an event and a significant increase in trigger rate can result from multiple interactions. The CDF calorimeter trigger was upgraded to solve the problems associated with data-taking at high luminosities [71]. At Level 2, the triggers require either:

- MET28: $\mathbb{E}_{T}>28 \mathrm{GeV}$;

- TWO JET3: at least two jets $(|\eta|<3.6)$ with $E_{T}>3 \mathrm{GeV}$;

- CJET20: at least one central jet $(|\eta|<1.1)$ with $E_{T}>20 \mathrm{GeV}$;

- MET30: $\mathbb{E}_{T}>30 \mathrm{GeV}$;

- TWO JET15: at least two jets $(|\eta|<3.6)$ with $E_{T}>15 \mathrm{GeV}$;

- MET40: $\mathbb{E}_{T}>40 \mathrm{GeV}$.

As luminosity increases a pre-scale ${ }^{1}$ is applied to all the above selections to limit the rate. For the MET30, CJET20 and TWO JET15 selections, a dynamic pre-scale ${ }^{2}$ (DPS) between 1 and 20 is chosen.

\footnotetext{
${ }^{1}$ A pre-scaled trigger operating with a rate $P_{R}$ records only $1 / P_{R}$ events.

${ }^{2} \mathrm{~A}$ dynamic prescale consists of a feedback loop that adjusts the pre-scale dynamically according to the total trigger rate.
} 
Level 3 exploits the full detector information, leading to a much improved reconstruction of the physics objects. Additional thresholds on $\mathbb{E}_{T}$ are applied for the three $\mathbb{E}_{T}$-triggers used in this thesis.

The requirements at the three trigger levels that define the different trigger paths are summarized in Table 4.1.

Table 4.1 - The $\mathbb{E}_{T}$-triggers used in this thesis.

\begin{tabular}{l|ccc}
\hline \hline Trigger paths & Level 1 & Level 2 & Level 3 \\
\hline MET_DIJET & JET10, MET28 & MET28, TWO JET3 & $E_{T}>30 \mathrm{GeV}$ \\
MET35_CJET & MET28 & MET30, CJET20, JET15, DPS & $35 \mathrm{GeV}$ \\
MET40/45 & MET28 & MET40 & $E_{T}>40 / 45 \mathrm{GeV}$ \\
\hline \hline
\end{tabular}

The MET_ DIJET trigger was introduced after the completion of the Level 2 trigger upgrade. It works at higher luminosities and collects events with $\mathbb{E}_{T}$ and at least two jets. This is the most efficient trigger selecting events in the analyses presented here.

The MET35_CJET trigger is the most common trigger used for analyses requiring $\mathbb{E}_{T}$ and at least two jets. Since it was implemented before the CDF calorimeter trigger upgrade, the requirements on $E_{T}$ and jet $E_{T}$ are tighter compared to the MET_DIJET trigger.

The MET_40/45 trigger is built by applying thresholds on $\mathbb{E}_{T}$ at the three levels of the trigger. No additional requirement are made.

The event selection at the trigger level relies on the online object reconstruction, while the data analysis is performed using objects reconstructed offline. Comparisons with experimental data show that the most accurate description of the simulated physics processes and the detector response is provided by the offline quantities. Therefore, the trigger efficiency needs to be parametrized as a function of offline quantities in order to have an estimate of the trigger acceptance for simulated physics processes.

A trigger efficiency parametrization based on a neural-network multivariate algorithm has recently been developed [72] to improve the modelling of the trigger turn-on outside of the fully efficient region with $E_{T}>50 \mathrm{GeV}$ and jet $E_{T}>30 \mathrm{GeV}$. This allows for a lower $E_{T}$ requirement in the event selection, increasing significantly the acceptance for the signal. The uncertainty of the neural network parametrization is estimated by the application of this technique to two different data samples: the high $p_{T}$ muon sample, where a reconstructed muon is required, and the JET-50 sample, which contains events with jets with $E_{T}>50 \mathrm{GeV}$. The MUON or BHMU sample is composed by high- $p_{T}$ muon events that are used to test the response of the trigger parametrization in the case of an expected imbalance of calorimeter $\mathbb{E}_{T}{ }^{3}$; the case in which the $\mathbb{E}_{T}$ is produced by mismea-

\footnotetext{
${ }^{3}$ The $\mathbb{E}_{T}$ is calculated at Level 2 and is not corrected for the presence of muons.
} 
surement of jet energies is considered by applying the trigger parametrization to events from the JET-50 sample. The trigger efficiency ranges from $40 \%$ for events with $\mathbb{E}_{T}=35$ $\mathrm{GeV}$ to $100 \%$ for events with $\mathbb{E}_{T}>50 \mathrm{GeV}$, as shown in Figure 4.1 .
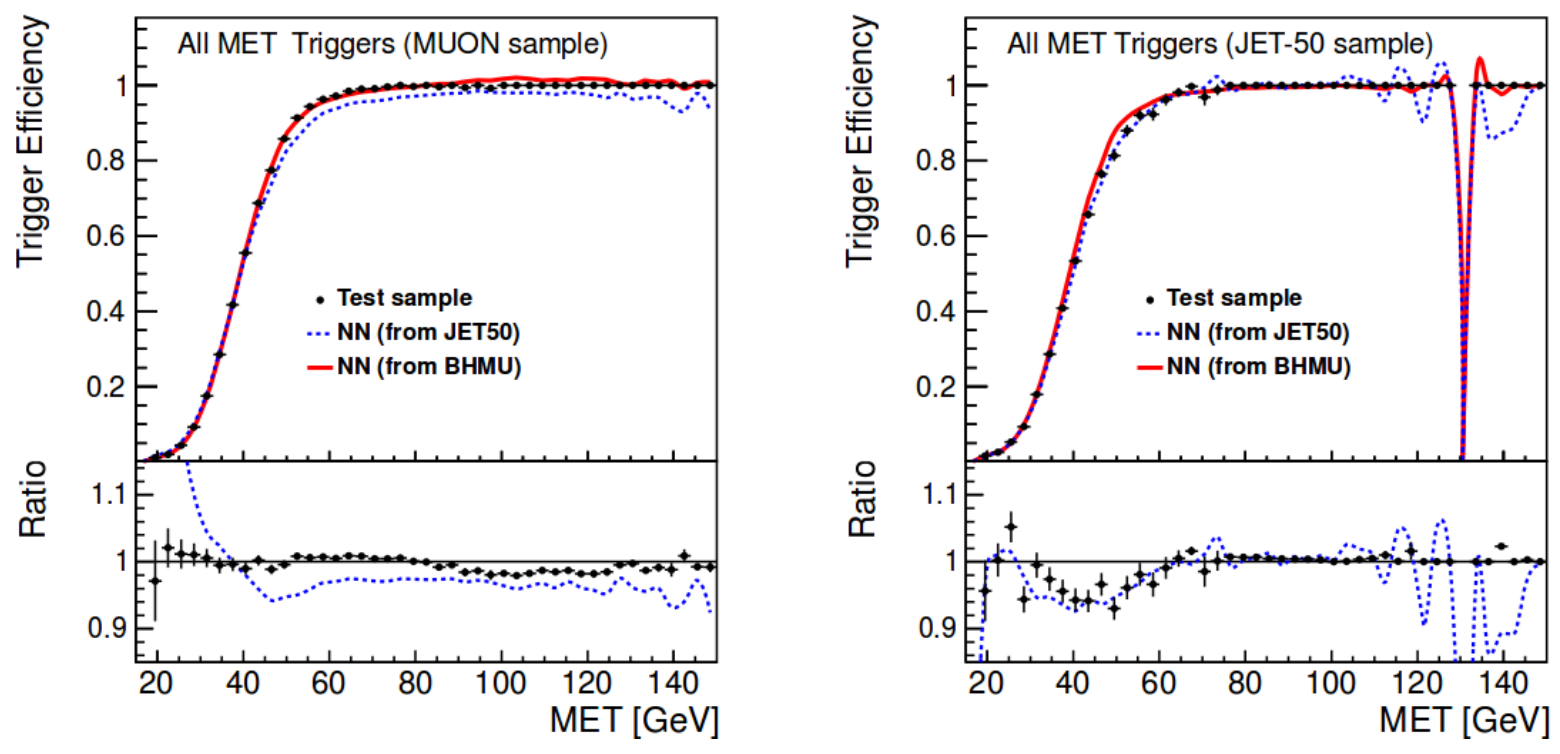

Figure 4.1 - Trigger efficiency for the $\mathbb{E}_{T}$-triggers as a function of $\mathbb{E}_{T}$ parametrized by a neuralnetwork function in the (left) MUON and (right) JET-50 samples. The agreement of the MUON and JET-50 parameterizations in the samples where they are derived is simply a consistency check. From [72].

\subsubsection{Offline Requirements and Control Regions}

The background model is derived from a combination of Monte Carlo (MC) simulations and data-driven methods. It is very important to validate the background model in different kinematic regions and for a variety of kinematic variables, especially when the signal we are searching for has a small cross section. Kinematic regions where the signal is negligible can be defined. For those regions, the data should be reproduced by the background model otherwise any discrepancy in the signal region could be a result of the imperfect modelling of the background processes. Furthermore, when using multivariate methods, all the input variables should be validated.

There are common selections between the s-channel single-top-quark search and the $W^{\prime}$ boson search presented in this thesis, as well as similarities in control region definitions. Events with two high- $E_{T}$ jets and $E_{T}$ are selected. Some of these events consist of $s-$ channel single top quark or $W^{\prime} \rightarrow t b$ candidates where $t \rightarrow W b \rightarrow \ell v$ and $\ell$ is an electron or a muon that is not identified in the detector or a $\tau$ lepton that is reconstructed as a jet in the calorimeters. To increase the acceptance for events with an unidentified $\tau$ lepton, events with three jets are also accepted. To suppress inclusive QCD multijet (MJ) production, events with four or more reconstructed jets are rejected. Specifically, we apply the following cuts: 
- $E_{T}>35 \mathrm{GeV}$ (both L5 and H1 corrected $\mathbb{E}_{T}$ );

- $E_{T}^{j 1}>25 \mathrm{GeV}, E_{T}^{j 2}>20 \mathrm{GeV}$ (jet energy thresholds are applied to both the L5corrected jet and the H1-corrected jet);

- No more than three jets with $E_{T}>15 \mathrm{GeV}$.

In addiction to this selection, further cuts are also applied:

- $\left|\eta^{j i}\right|<2.4 ;\left|\eta^{j 1}\right|$ and $\left|\eta^{j 2}\right|<2$; and $\left|\eta^{j 1}\right|$ or $\left|\eta^{j 2}\right|<0.9$ (to ensure that the two highest $E_{T}$ jets are within the silicon-detector acceptance);

- For data, we also apply the $E_{T}$ clean-up cuts, which include removing halo events and events with jets in the chimney area.

We further separate the events passing the above criteria into signal-sensitive regions (Preselection and, in the $W^{\prime}$ boson search, EWK) and control regions (TRM, QCD-rich, and, in the s-channel single top quark search, EWK ). The regions are defined as follows:

- TRM (Tag Rate Matrix): a QCD multijet dominated region, used for the QCD multijet data-derived model (see Section 4.3.3) and to perform validation.

- $\Delta \phi\left(\mathbb{E}_{T}, j_{2}\right)<0.4$

- $35<\mathbb{E}_{T}<70 \mathrm{GeV}$.

- QCD-rich: an independent QCD multijet dominated region, used to validate the QCD multijet data-derived model.

$-\Delta \phi\left(\mathbb{E}_{T}, j_{2}\right)<0.4$

- $\mathbb{E}_{T}>70 \mathrm{GeV}$.

- EWK/Top (hereinafter referred to simply as EWK): a region that requires the presence of at least one high $p_{T}$ lepton. It is dominated by electroweak and top processes, which are modelled with MC. In the s-channel analysis, it is used to validate the MC model as well as the QCD model; in the $W^{\prime}$ boson analysis, events accepted in this region are further analyzed as potential signal events.

- $\Delta \phi\left(\mathbb{E}_{T}, j_{2,3}\right)>0.4, \Delta \phi\left(\mathbb{E}_{T}, j_{1}\right)>1.5$

- identified electron or muon with $p_{T}>20 \mathrm{GeV} / c$.

- Preselection (or Signal Box): signal-sensitive region that vetoes events dominantly due to QCD multijets, as well as events with leptons. 
- QCD multijet background events most often contain $\mathbb{E}_{T}$ generated through jet energy mismeasurements. Neutrinos produced in semileptonic $b$-hadron decays can also contribute to the $\mathbb{E}_{T}$ of these events. In both cases, the $\vec{E}_{T}$ is typically aligned with the least energetic jet, and events are rejected by requiring $\Delta \phi\left(\mathbb{E}_{T}, j_{2,3}\right)>0.4, \Delta \phi\left(\mathbb{E}_{T}, j_{1}\right)>1.5$;

- no identified electron or muon with $p_{T}>20 \mathrm{GeV} / c$.

The definition of the regions is summarized in Figure 4.2.

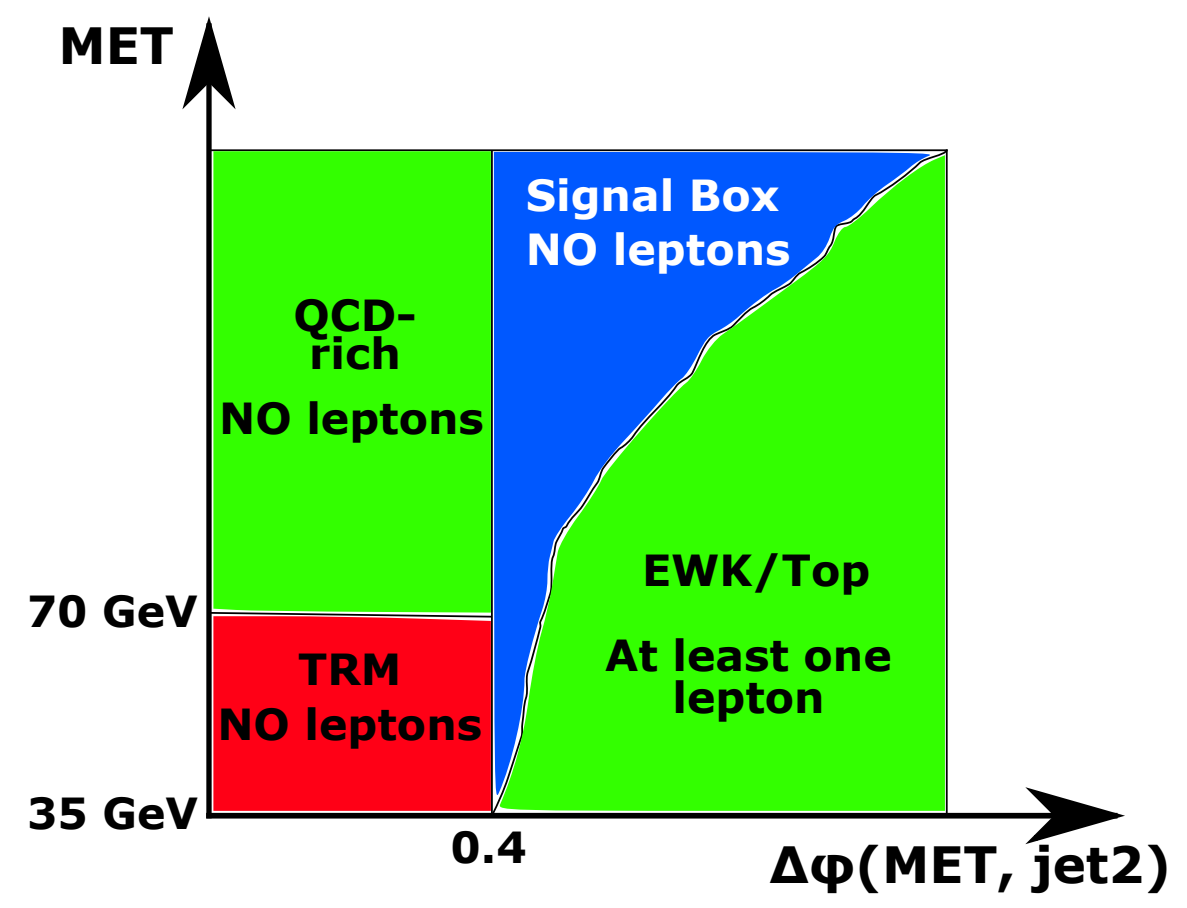

Figure 4.2 - The kinematic regions defined for events with two high- $E_{T}$ jets and $\mathbb{E}_{T}$.

\subsection{Background Model}

In the searches for s-channel single top quark production and for $W^{\prime}$ boson production, events with energetic jets and large missing transverse energy are selected. Many other SM processes can mimic this final state and are considered as backgrounds in the searches. Among these processes are QCD multijet production, $W / Z$ bosons produced in association with additional jets ( $V+$ jets, where $V$ stands for a $W$ or a $Z$ boson), top-quark (single in the $t$-channel and, in the case of the $W^{\prime}$ boson analysis, in the $s$-channel, and pair, denoted as $t \bar{t})$ production, and diboson $(W W, W Z$, and $Z Z$, also indicated as $V V$ ) production. Given the Higgs boson discovery, the associated production of a Higgs boson and a $W$ or $Z$ boson $(V H)$ is also included.

The most important contribution to the preselection sample, as defined in Section 4.2.2, is due to QCD multijet production, followed by $V+$ jets, top, $V V$ and $V H$ production. All 


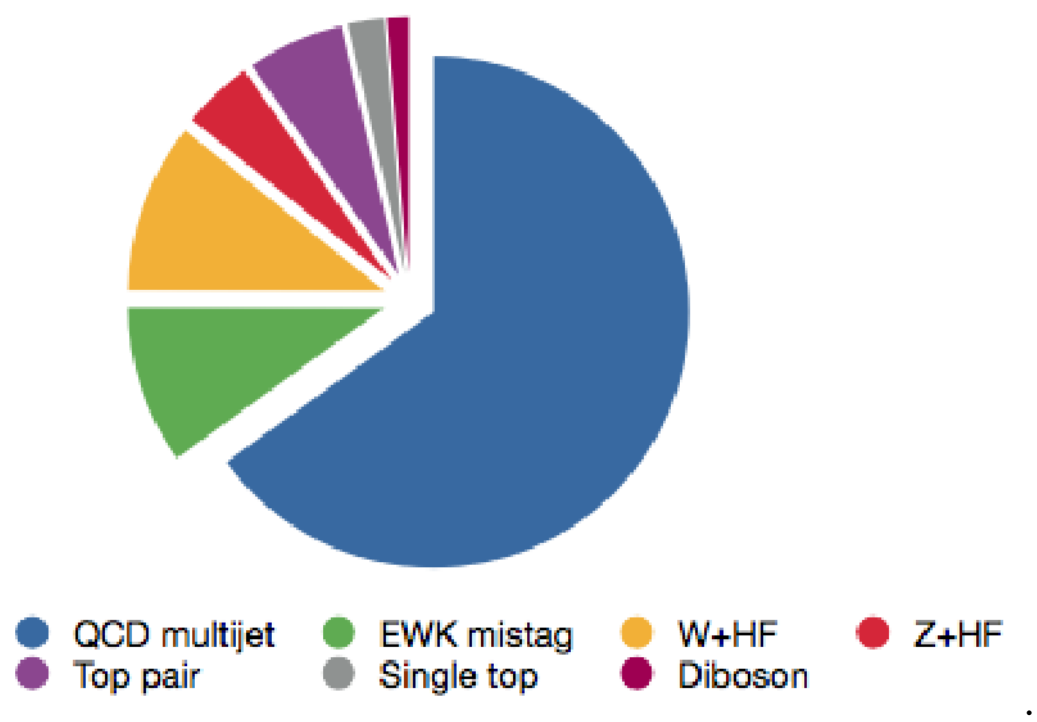

Figure 4.3 - Schematic view of contribution from the SM processes to the selected data sample. The $V H$ production is not shown.

processes other than QCD multijet production are denoted as EWK (even though some of them, e.g., $t \bar{t}$ production, can be produced via the strong interaction). This notation is chosen since the QCD multijet background is mainly instrumental with mismeasured $\mathbb{E}_{T}$, while the EWK processes have $\mathbb{E}_{T}$ in the final state due to the production of neutrinos.

Events are selected with at least one $b$-tagged jet. Each background process can be accompanied by the production of heavy-flavour (HF) $b$-tagged jets, and light-flavour (LF) falsely tagged (mistagged) jets. The EWK HF production is modelled with MC simulation, while the mistags are modelled with simulated EWK LF events weighted by a mistag matrix derived from data. For the QCD multijet background a data-driven Tag Rate Matrix method is applied that models both HF production and mistags from LF. Figure 4.4 shows a sketch that summarizes the strategy used to model the background processes.

\subsubsection{EWK Heavy-Flavour Background Model}

Since kinematic properties of events associated with $V+$ jets, top, $V V$ and $V H$ production are relatively well understood, they are modelled using $\mathrm{MC}$ simulations.

The ALPGEN generator [73] is used to model $V+$ jets production ${ }^{4}$. The processes are split by the boson decay channels and the number of associated partons (quarks or gluons). Montecarlo samples are generated with ALPGEN to describe $W(\rightarrow l v)+b \bar{b}+X, W(\rightarrow$ $l v)+c \bar{c}+X, W(\rightarrow l v)+c+X$, and $W(\rightarrow l v)+X$, where $X$ is the additional parton(s). Similarly samples for $Z(\rightarrow v v / \ell \ell)+b \bar{b}+X, Z(\rightarrow v v / \ell \ell)+c \bar{c}+X$, and $Z(\rightarrow v v / \ell \ell)+X$ are generated. A sample of $Z(\rightarrow b \bar{b})+X$ is also generated with PYTHIA. The normaliza-

\footnotetext{
${ }^{4}$ For the ALPGEN matrix element the CTEQ5L parton distribution function is used as input.
} 


\section{QCD EWK}

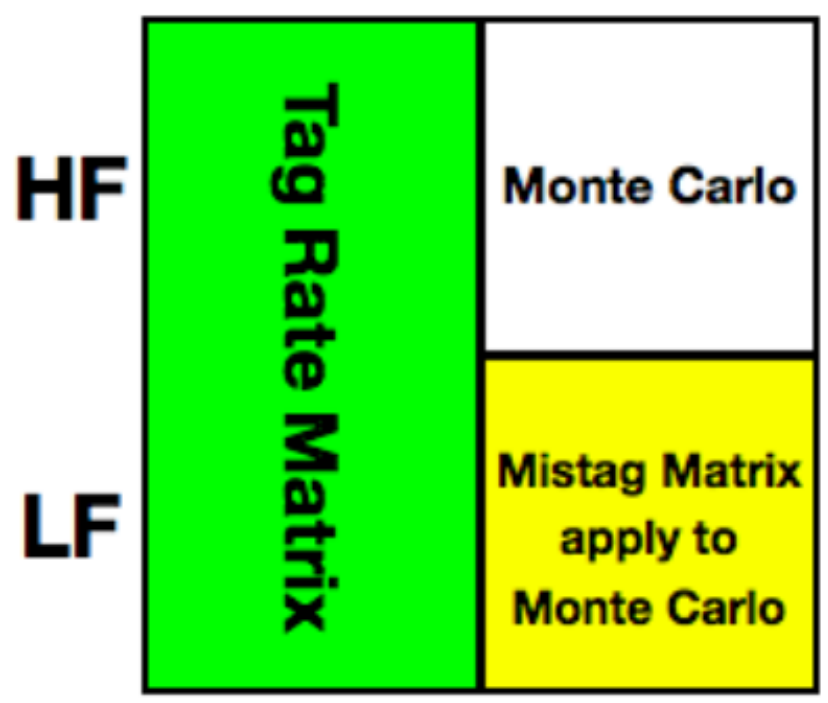

Figure 4.4 - Illustration of the strategy for the background model.

tion for $W+c$ production is taken from the measured cross section [77] while all the other processes are normalized to the leading order (LO) with up to four partons and combined generator-to-reconstructed-jet matching $[75,76]$. The HF fraction provided by the ALPGEN cross section is corrected with a scale factor based on the number of events observed in independent data control samples, following a procedure similar to the one described in Ref. [78]. A different scale factor is applied in the single-tag and in the double-tag samples to take into account the enhancement in the single-tag sample due to $V+$ twoHF-quark events in which both the jets originating from the two HF quarks are clustered into a single jet and an extra jet is produced by radiation.

The PYTHIA generator is used to model $V V, V H$, and $t \bar{t}$ pair production ${ }^{5}$. Normalizations are taken from theoretical cross section predictions $[79,80,16]$. The boson decays are set to be inclusive.

For the $t$-channel single top quark production, as well as for the $s$-channel (considered as a background in the $W^{\prime}$ boson analysis), the POWHEG [81] generator is used, and is normalized to the NNLO cross section [22] taking an NLO PDF as input to the simulation.

Parton showering and hadronisation are simulated in all cases with PYTHIA, using the CTEQ5L parton distribution function [82] as input tuned to the Tevatron underlyingevent data [83]. Event modelling also includes simulation of the detector response using GEANT [84] or a parameterization based on GEANT [85].

\footnotetext{
${ }^{5}$ The POWHEG generator was tested for $t \bar{t}$ production, showing no significant deviation from the kinematic distributions generated by PYTHIA and used in the analyses presented in this thesis.
} 


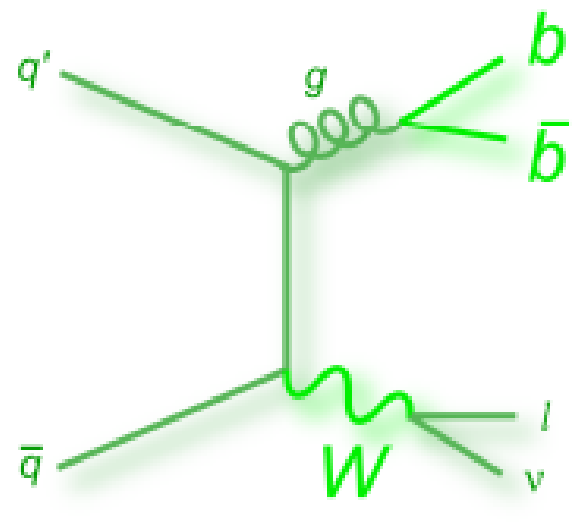

(a) $W+b \bar{b}$

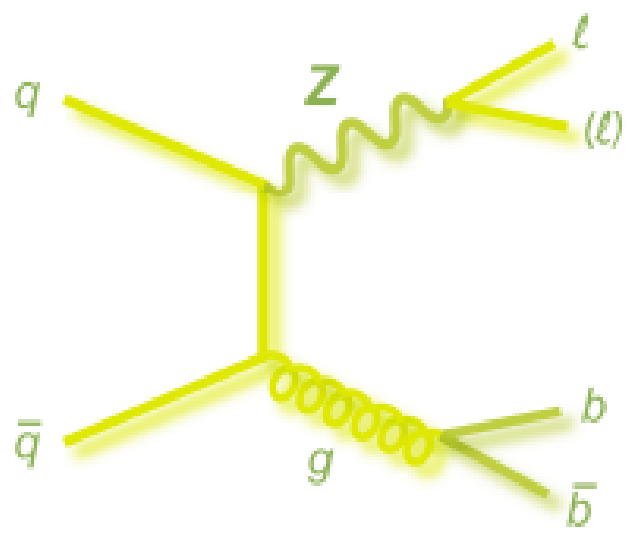

(b) $Z+\bar{b}$

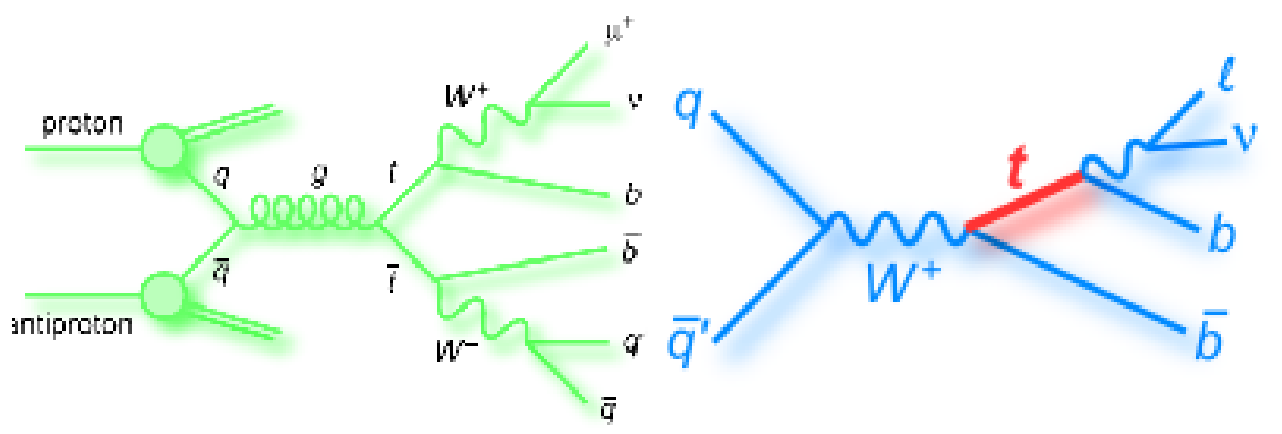

(c) Top-quark pair production

(d) Single top-quark production (schannel)

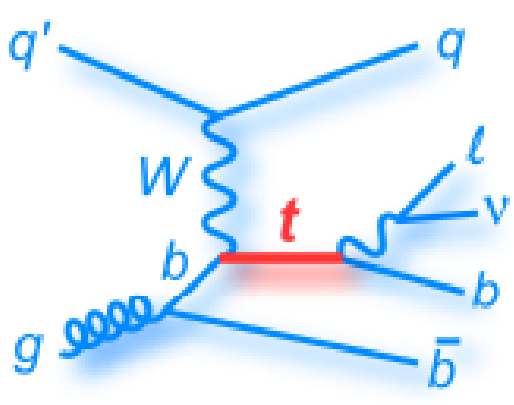

(e) Single top ( $t$-channel)

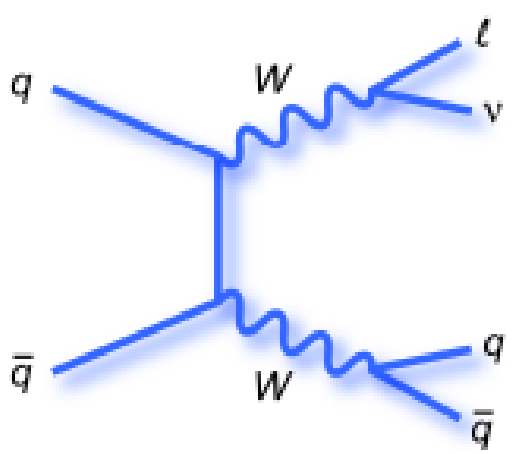

(f) $W W$ pair

Figure 4.5 - Representative Feynman diagrams for: (a) $W+\mathrm{HF}$, (b) $\mathrm{Z}+\mathrm{HF}$, (c) $t \bar{t}$, (d) s-channel single top, (e) $t$-channel single top, and (f) $W W$ production.

When using MC to estimate backgrounds containing $b$-quarks, the $b$-tagged jet is required to originate from a HF ( $b$ or $c$ ) quark at the generator level. The mistag LF contribution is estimated using the data-driven mistag model described in the next Section. 


\subsubsection{EWK Light-Flavour Mistag Model}

Due to the tracking resolution or the presence of long-lived particles such as $K_{S}$ or $\Lambda$, a light-flavour jet can be falsely $b$-tagged. The contribution to the background coming from mistags is classified in two categories: mistags from QCD multijet event, which will be discussed in Section 4.3.3, and mistags from EWK processes, which are described in this Section.

The mistag rate for light-flavour jets is estimated from data. The mistag models for SECVTX, JETPROB, and HOBIT are based on the application of multi-dimensional matrices parameterizing the mistag rate as a function of different kinematic quantities and calculating it for each bin of data.

\section{SECVTX and JETPROB Mistag Matrices}

As described in Sections 3.2.1 and 3.2.2, the SECVTX and JETPROB algorithms identify a jet originating from a $b$-quark using the two-dimensional decay length $L_{2 D}$. The sign of $L_{2 D}$ defines positively or negatively tagged jets.

The mistag probability for light-flavour jets is a result of tracking resolution, which causes a non-zero width of the $L_{2 D}$ distribution. While large positive $L_{2 D}$ values are expected for $b$-jets, the $L_{2 D}$ distribution for light-flavour jets peaks at zero. The chance of positively tagging or negatively tagging a light-flavour jet is about equal and the negatively tagged jets are thus used by both SECVTX and JETPROB to estimate the positive mistag rate.

A set of mistag rate matrices is derived from high $E_{T}$ jet data. The matrices are defined in bins of kinematic variables such as jet $E_{T}$ and $\eta$, the number of good tracks in a jet, the number of good vertices, and $z$ position of the primary vertex. The mistag rate is calculated bin-by-bin as the ratio of the number of negatively tagged jets over the number of taggable jets ${ }^{6}$. Additional asymmetry factors are applied to correct for the bias due to long-lived particles in light-flavour jets (e.g., $K_{L}$ ) [86]. The negative tag rates vary from period to period, and the standard parameterization does not predict them perfectly. The matrices that match the data analyzed in this thesis are used.

\section{HOBIT Mistag Matrix}

Since the HOBIT algorithm tags jets using a machine learning method, there are no negative tags that can be exploited to estimate the mistag rate. To measure simultaneously the $b$-tagging efficiency and the mistag rate, two new techniques are applied and combined:

\footnotetext{
${ }^{6}$ SECVTX (JETPROB) define a jet with more than two (one) good displaced tracks as a taggable jet. HOBIT defines a jet with at least one good SECVTX track as taggable
} 
the $t \bar{t}$ cross section method and the electron conversion method [63].

The $t \bar{t}$ cross section method calibrates the predicted $b$-tagging efficiency and mistag rate in MC using $t \bar{t}$ data events in a $W+\geq 3$-jets sample, assuming the $t \bar{t}$ cross section as known. A two-dimensional fit to data is performed as a function of the $b$-tag and the mistag scale factors. The tag rate probability is parameterized with a 5-dimensional matrix, where each element is the measured tag rate in a bin of jet $E_{T}$, jet $\eta$, the number of tracks in the jet, the number of primary vertices in the event, and the $z$ location of the primary vertex. Separate matrices are constructed for $b_{-}, c_{-}$, and light-flavour jets and for each HOBIT operating point. The fitted $b$-tag and mistag scale factors are shown in Figures 4.6 and 4.7, respectively, as functions of the minimum HOBIT output value. The curves represent a linear fit to the $b$-tag scale factor and a parabolic fit to the mistag scale factor as a function of the minimum HOBIT output value. The variation due to the uncertainty on the $t \bar{t}$ cross section is also shown.

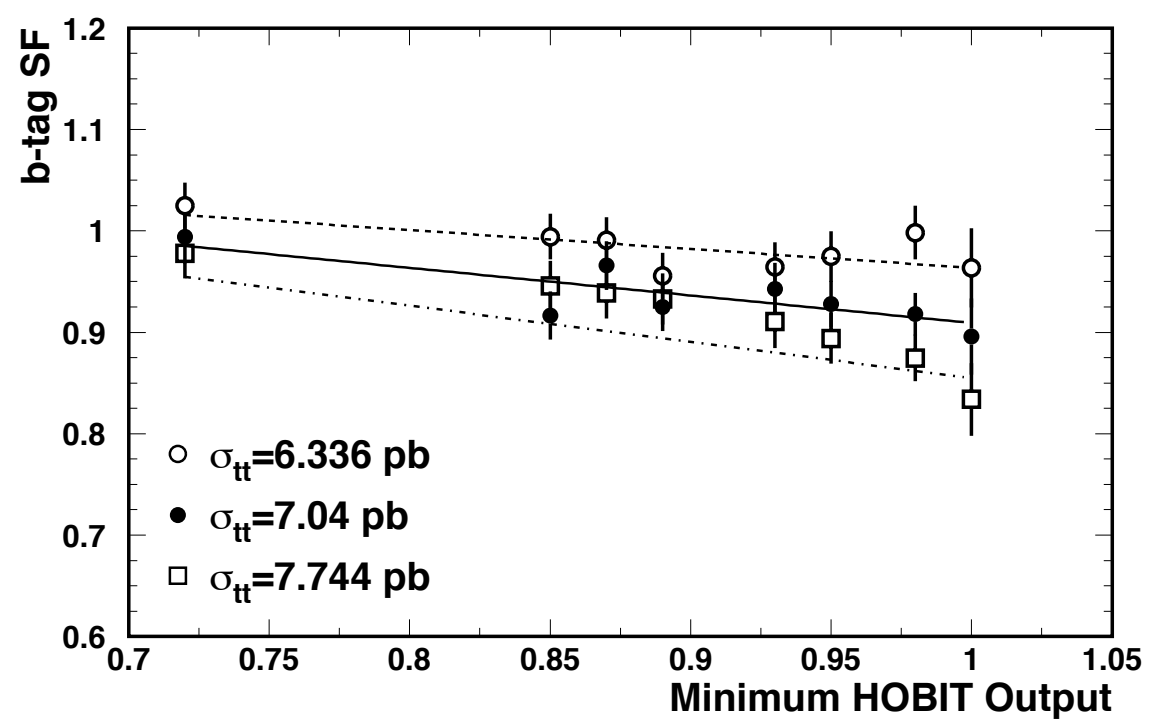

Figure 4.6 - The measured value of the $b$-tag scale factor for the HOBIT tagger as a function of the minimum HOBIT output value. Variations are shown assuming two values of the $t \bar{t}$ cross section. The straight lines are fits to the scale factors assuming the central value of the $t \bar{t}$ cross section $\left(\sigma_{t \bar{t}}=7.04 \mathrm{pb}\right)$, and $\sigma_{t \bar{t}}=6.336 \mathrm{pb}$, the more conservative case for the purpose of estimating uncertainties. The latter fit has been reflected through the central line to obtain a symmetric uncertainty band. From [63].

The electron conversion method takes advantage of the enhancement of heavy-flavour jets among the jets containing electrons. The $b$-tag and mistag scale factors are estimated using a dijet-event sample with one jet containing an electron candidate, discriminating between heavy- and light-flavour jets based upon whether the electron is identified as coming from a photon conversion.

The two methods are eventually combined to extract a $b$-tag scale factor of $0.993 \pm 0.032$ for loose HOBIT tags (HOBIT output between 0.72 and 0.98 , see Section 3.2.3) and 0.937 


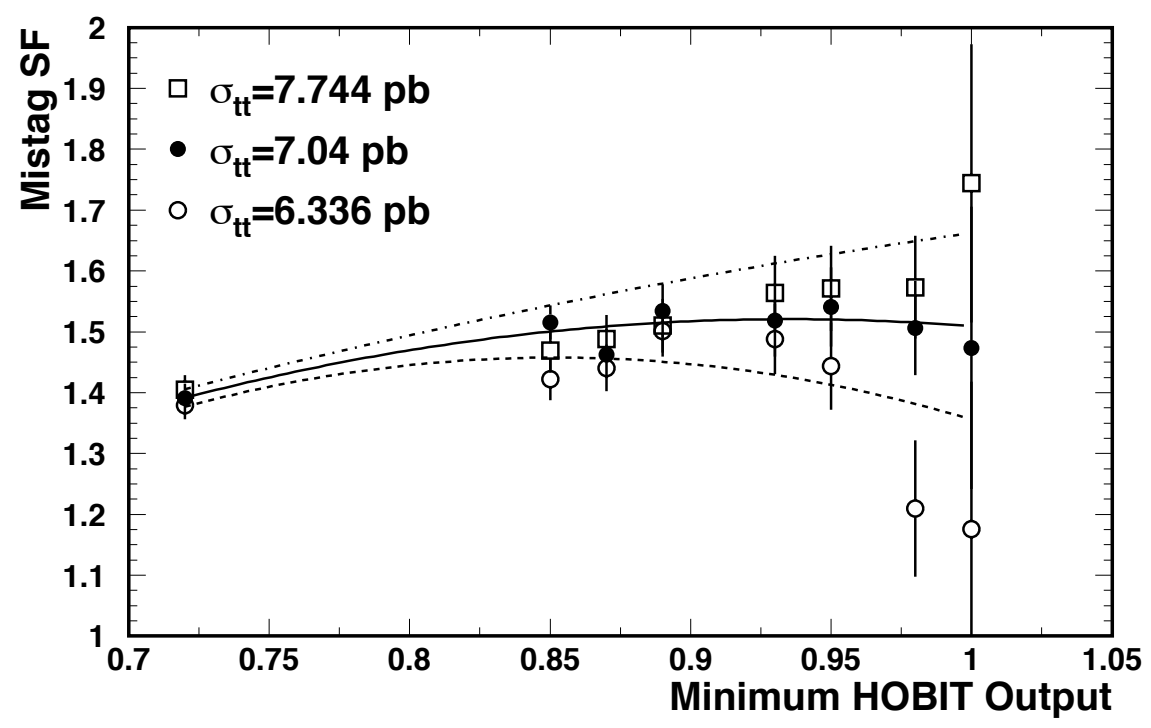

Figure 4.7 - The measured value of the mistag scale factor for the HOBIT tagger as a function of the minimum HOBIT output value. Variations are shown assuming two values of the $t \bar{t}$ cross section. Parabolas are fit to the results assuming the central value of the $t \bar{t}$ cross section $\left(\sigma_{t \bar{t}}=7.04 \mathrm{pb}\right)$, and for $\sigma_{t \bar{t}}=6.336 \mathrm{pb}$. The latter has been reflected through the curve for the central value to obtain the depicted uncertainty band. From [63].

\pm 0.037 for tight HOBIT tags (HOBIT output greater than 0.98 ). The combination results in an improvement of more than $25 \%$ in the $b$-tag scale factor uncertainty compared to SECVTX. The combined results for the mistag scale factors are $1.331 \pm 0.130$ for tight HOBIT tags, and $1.492 \pm 0.277$ for loose HOBIT tags.

\section{Mistag Matrix Application}

The mistag matrices return per-jet mistag rate probabilities that are recombined as follows to obtain per-event mistag rate probabilities for different event categories based on tagging requirements:

$$
\begin{aligned}
& \text { mistag rate probability }{ }_{1 T}=M_{S_{j 1}} \times\left(1-M_{S_{j 2}}\right)+M_{S_{j 2}} \times\left(1-M_{S_{j 1}}\right) \\
& \text { mistag rate probability }{ }_{T T}=M_{S_{j 1}} \times M_{S_{j 2}} \\
& \text { mistag rate probability } T_{L}=M_{S_{j 1}} \times\left(1-M_{S_{j 2}}\right) \times M_{J_{j 2}}+M_{S_{j 2}} \times\left(1-M_{S_{j 1}}\right) \times M_{j_{j 1}}
\end{aligned}
$$

where $1 \mathrm{~T}$ is the category with one jet tagged by SECVTX and one non-tagged jet, TT is the category with both the jets tagged by SECVTX, and TL is the category with one jet tagged by SECVTX and the other tagged by JETPROB but not by SECVTX. $M_{S_{j i}}\left(M_{J_{j i}}\right)$ is the SECVTX (JETPROB) mistag rate probability for jet $j_{i}$. 
For the tagging categories defined by HOBIT:

$$
\begin{aligned}
& \text { mistag rate probability }_{1 T}=M_{T_{j 1}} \times\left(1-M_{L_{j 2}}\right)+M_{T_{j 2}} \times\left(1-M_{L_{j 1}}\right) \\
& {\text { mistag rate probability } T_{T}}^{=} M_{T_{j 1}} \times M_{T_{j 2}} \\
& \text { mistag rate probability }_{T L}=M_{T_{j 1}} \times\left(M_{L_{j 2}}-M_{T_{j 2}}\right)+M_{T_{j 2}} \times\left(M_{L_{j 1}}-M_{T_{j 1}}\right)
\end{aligned}
$$

where $\mathrm{T}$ and $\mathrm{L}$ in subscripts stand for the tight HOBIT cut and loose HOBIT cut, so that the notations $1 \mathrm{~T}, \mathrm{TT}$, and TL, define events with one tight, two tight, and one tight and one loose HOBIT tags, respectively. $M_{T_{j i}}$ and $M_{L_{j i}}$ are the mistag rate probabilities for the tight and loose tagged HOBIT jets.

Only mistags from EWK samples with both the leading jets originating from light-flavour quarks are included in the mistag rate estimates shown above. In the analysis, mistags for double tagged events that are promoted from the single tagging categories are also considered, so that single tagged (1T) events with the non-tagged light-flavour jet weighed by its mistag rate probability are also included among the mistag-TT events. Mistag rate probabilities are applied only to light-flavour MC samples, which include $V+\mathrm{LF}$ and $V V$ events, while the contribution from top events is considered negligible. In case of mistagTT events promoted from single tagged events, the contribution from $V+\mathrm{HF}$ with one jet matched to a $b$-quark at generator level and the other jet originating from a light-flavour quark is also included.

\subsubsection{QCD Multijet Model}

The missing transverse energy detected in QCD multijet events does not result from the production of undetectable particles, as shown in Figure 4.8.

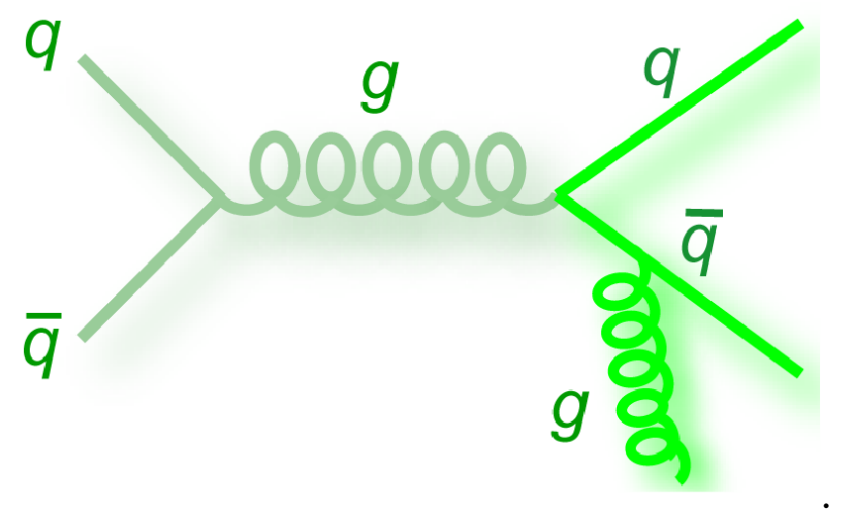

Figure 4.8 - Feynman diagrams for QCD multijet production. Usually no neutrino is present, except when it is produced in the decay of a hadron. 
For example, in a QCD dijet event the two jets should carry the same amount of transverse momentum such that the total transverse momentum of the event balances. The limited calorimeter energy resolution, of the order of $10 \%$ for jets with $E_{T}$ of $50 \mathrm{GeV}$, or the energy loss in uninstrumented regions of the detector, can cause mismeasurements of jet energies that result in an imbalance of the transverse momentum. Even though the $\mathbb{E}_{T}$ arises mainly from mismeasurements, the QCD multijet production rate is ten orders of magnitude larger than the sum of the other SM processes, dominating the data sample of events with large missing transverse energy and jets. Simulation of QCD multijet production with large $\mathbb{E}_{T}$ is challenging due to the large production rate and the large theoretical uncertainties. In this thesis, a data-driven Tag Rate Matrix method is developed and applied. Only tagged QCD multijet events are modelled by the method since both the single top and the $W^{\prime}$ boson analyses require $b$-tagged jets.

The tag rate matrix (TRM) method estimates the probability for a QCD multijet event to be either 1T, TL, or TT, with the definition of the notation changing according to the tagger used in the analysis, as discussed in the previous Section. The the QCD multijet tag rate probability is derived in the TRM control region defined in Section 4.2.2 and applied as a weight to pretag data satisfying our analysis selection criteria to build a model of the tagged QCD multijet background. The QCD-rich and EWK control regions are used to validate the TRM method, along with the model for the other EWK processes.

A tag rate matrix is constructed as a 4-D matrix, where each matrix element is the measured rate in a bin of the following variables:

- $H_{T}$ : the scalar sum $E_{T}$ of all the reconstructed jets;

- $p_{T}$ : the imbalance of transverse momentum of tracks;

- $j_{1,2} \mathrm{Z}$ : ratio of the sum $p_{T}$ of the tracks that pass the SECVTX pass1 selections to the jet transverse energy, for both $j_{1}$ and $j_{2}$.

The first two variables, $H_{T}$ and $p_{T}$, are event variables, while the $j_{i} \mathrm{Z}$ is a jet variable. The bin boundaries of the four dimensions of the matrix are:

$$
\begin{array}{rl}
H_{T} & \in[45,80,100,120,150,200,1000] \mathrm{GeV} \\
p_{T} & \in[0,8,15,25,35,45,60,1000] \mathrm{GeV} / c \\
j_{1} \quad Z & \in[0,0.025,0.05,0.1,0.15,0.2,0.3,0.4,0.6,0.8,10.0] \\
j_{2} & Z
\end{array}
$$

The TRM for the HOBIT categories has 2 extra dimensions, $N_{v t x}$ and $p_{\perp}^{\mu}$. 
- $N_{v t x}$ : number of reconstructed vertices;

- The "muon pt-rel" $p_{\perp}^{\mu} \equiv p_{\mu 1} \sin \left(\hat{\mu}_{1} \cdot \hat{j}_{1}\right)+p_{\mu 2} \sin \left(\hat{\mu}_{2} \cdot \hat{j}_{2}\right)$, where $p_{\mu i}$ represents the momentum of the most-energetic muon within the cone of jet $i$, and $\sin \left(\hat{\mu}_{i} \cdot \hat{j}_{i}\right)$ is the sine of the angle between the muon and jet directions.

The $p_{\perp}^{\mu}$ variable is an input to the HOBIT algorithm. Jets with large $p_{\perp}^{\mu}$ values tend to have a high probability of being tagged by the HOBIT tagger because $b$ quarks tend to decay semileptonically roughly $10 \%$ of the time and the HOBIT assumes that jets with a significant $p_{\perp}^{\mu}$ value arise from those decay.

The bin boundaries of these additional two dimensions are:

$$
\begin{aligned}
p_{\perp}^{\mu} & \in\left[1 \times 10^{-5}, 1 \times 10^{-4}, 0.3,0.6,0.9,1.2,1.4,1.7,2.0,100\right] \mathrm{GeV} / c \\
N_{v t x} & \in[0.5,1.5,2.5,3.5,100]
\end{aligned}
$$

Two matrices are filled for each tagging category, one to count taggable events and one to count tagged events. The tagging rate is calculated for each element of these two matrices:

$$
\text { tag rate probability }=\frac{\text { number of tagged events }}{\text { number of taggable events }}
$$

When applying the TRM in the control regions, we weight the pretag data with the calculated tag rate and subtract the weighted contributions from EWK processes.

QCD multijet $=($ tag rate probability $\times$ pretag data - tag rate probability $\times$ pretag electroweak $) \times S F$

The QCD multijet normalization is calculated as a scale factor (SF) on top of the prediction given by the application of the TRM method. The SF is calculated individually for each tagging category and defined as:

$$
S F=\frac{\text { data }- \text { electroweak }}{\text { tag rate probability } \times \text { pretag data }- \text { tag rate probability } \times \text { pretag electroweak }}
$$

The uncertainty on the SF is:

$$
\begin{aligned}
\left(\frac{\delta S F}{S F}\right)^{2} & \simeq\left(\frac{\delta(\text { data }- \text { electroweak })}{\text { data }- \text { electroweak }}\right)^{2}+ \\
& \left(\frac{\delta(\text { tag rate probability } \times \text { pretag data }- \text { tag rate probability } \times \text { pretag electroweak })}{(\text { tag rate probability } \times \text { pretag data }- \text { tag rate probability } \times \text { pretag electroweak })}\right)^{2}
\end{aligned}
$$

The systematic uncertainties from EWK HF production and mistagged EWK LF production are included in the calculation of the SF uncertainty, in order to properly propagate all the uncertainties to the determination of the QCD multijet background. 
To maintain a blind analysis, the QCD multijet normalization in the signal region is obtained from a SF calculated as above but derived in an independent sample, composed of events rejected when applying the full event selection, as explained in Sections 6.1.2 and 7.2.

\subsection{Signal Model}

The signal model for the s-channel analysis has been described in Section 4.3.1. The signal model for the $W^{\prime}$ boson analysis is simulated using PYTHIA for $W^{\prime}$ boson masses $M_{W^{\prime}}$ in the range $300 \leq M_{W^{\prime}} \leq 900 \mathrm{GeV} / c^{2}$ in $100 \mathrm{GeV} / c^{2}$ increments considering purely righthanded decays. The interference between the $W$ boson and $W_{L}^{\prime}$ boson is model-dependent and constrained to be very small, so it can be neglected ${ }^{7}$. Under this assumption the lefthanded model is identical to a right-handed model where the decay to a right-handed neutrino $v_{R}$ is allowed. The two models show identical cross sections and branching ratios so that a single simulation can be used to represent both. If the decays to $v_{R}$ are forbidden, as it would be for a leptophobic $W^{\prime}$ or in the case of a $v_{R}$ that is more massive than the $W^{\prime}$ boson, the only effect is an increased branching ratio to $t b$. The same model can be used to represent both the right-handed cases, scaling the branching ratio to $t b$ accordingly. Our model assumes a narrow $W^{\prime}$ width, i.e. the width of the reconstructed resonance is determined by detector resolution rather than the intrinsic width.

\footnotetext{
${ }^{7}$ Ref. [87] shows that the destructive interference between the $W$ boson and the $W_{L}^{\prime}$ boson reduces the total cross-section across the considered mass range, thus this model is only considered for $W^{\prime}$ bosons with arbitrarily small coupling strength.
} 


\section{Chapter 5}

\section{Statistical Interpretation}

Testing theory predictions with experimental data is the key component of the scientific method. The observed data are compared to predictions of each relevant source of background, each source of signal, and the associated uncertainties. When the uncertainties associated to the background predictions are larger than the predicted signal rates, a comparison of the total data event counts to the total theory prediction (known as a 'counting experiment') is not sufficient to test the theory. In this case, searches require the comparison of data to prediction in intervals (bins) of a distribution chosen as a final discriminant. This allows the data to constrain the background rate and shape, increasing sensitivity to the signal. Achieving good separation of signal events from background events is crucial. Typically, the low signal-to-background bins have higher rate and serve to constrain the background rates. The predicted shape for each background allows an extrapolation into the signal-rich region, and the shape uncertainties determine the uncertainty on the extrapolation.

The compatibility of the theory with experimental data is estimated using statistical techniques. Two kinds of statistical analyses are performed. When looking for BSM physics, like in the $W^{\prime}$ boson search (see Chapter 7), in the absence of an observation we set an upper limit on the production cross section times branching ratio at $95 \%$ confidence level (C.L.). When probing known SM processes, like in the s-channel search, (see Chapter 6), the production cross section is directly measured, and $p$-values are computed to determine the significance of observation of the measured process. In these analyses the fits are performed using Bayesian techniques. Systematic uncertainties are handled with a Bayesian approach assigning flat priors to the nuisance parameters. Frequentist approaches are not used unless explicitly mentioned. 


\subsection{Bayesian Approach}

A bin is defined as a portion of the final discriminant variable space for which the number of expected events $\mu$ is compared to the number observed events $n$. The probability to observe the expected $\mu$ events given $n$ observed events, $\mathrm{P}(\mu \mid n)$, is evaluated bin-by-bin to quantify the agreement between the prediction and the data. Under the assumption of prior knowledge, Bayes' theorem [88] can be applied to evaluate $\mathrm{P}(\mu \mid n)$ as follows:

$$
P(\mu \mid n)=\frac{L(n \mid \mu) \pi(\mu)}{P(n)}
$$

where $\mathrm{P}(\mu \mid n)$ is the posterior probability on $\mu, \pi(\mu)$ is the prior probability on $\mu$, and $\mathrm{P}(n)$ is the probability to observe $n$ events. The statistical probability $\mathrm{L}(n \mid \mu)$ to measure $n$ events given the expected $\mu$ events is given by the Poisson distribution:

$$
L(n \mid \mu)=\frac{\mu^{n} e^{-\mu}}{n !}
$$

From equation (5.1), using the condition that the sum of all probabilities should sum to unity:

$$
\int P(n \mid \mu) d \mu=1=\int \frac{L(n \mid \mu) \pi(\mu)}{P(n)} d \mu
$$

one obtains:

$$
P(n)=\int L(n \mid \mu) \pi(\mu) d \mu
$$

Specifying $\mu$ in terms of the contribution from the expected number of signal $(s)$ and background $(b)$ events, one obtains:

$$
P(s+b \mid n)=\kappa(n, s+b) \frac{(s+b)^{n} e^{-(s+b)}}{n !}
$$

where $\kappa(n, s+b)=\pi(s+b) / P(n)$.

The number of SM background events is not a parameter of interest and is taken as given, i.e. $P(s \mid n, b)$. In order to know how $P(s \mid n, b)$ relates to the SM prediction of the signal $s_{\mathrm{SM}}$, the ratio $\beta=s / s_{\mathrm{SM}}$ is also introduced for the case of non-zero SM signal. A likelihood function $L$, that represents the probability for $\beta$ to have a value between 0 and $l$, given the observed number of events $n$, can be written as:

$$
L=\int_{0}^{l} P\left(\beta \mid n, \sum_{k} b_{k}, s_{S M}\right) d \beta
$$


where the total number of expected background events $b$ is expressed in terms of the expected event yields for each background process $b_{k}$.

Up to this point systematic uncertainties have not been taken into account. Systematic uncertainties affect the predicted event yields of both $s$ and $b_{k}$ as rate uncertainties, which apply to all the bins of a distribution with $100 \%$ positive correlation, and as shape uncertainties, which apply separately to each bin with varying degrees of correlation.

To each independent source of systematic uncertainty $m$ corresponds a nuisance parameter $\theta_{m}$. For a given set of nuisance parameter values, the number of expected events can be expressed as:

$$
\mu=\sum_{k}\left[\left(\prod_{m \in S} \theta_{m}\right) b_{k}\right]+\beta\left(\prod_{m \in S} \theta_{m}\right) s_{\mathrm{SM}}
$$

where $\mathcal{S}$ is the set of systematic uncertainties. To model each nuisance parameter, a Gaussian distribution is used:

$$
G\left(\theta_{m}>0 \mid \tilde{\mu}, \sigma\right)=\frac{1}{\sqrt{2 \pi \sigma^{2}}} \exp \left[-\frac{\left(\theta_{m}-\tilde{\mu}\right)^{2}}{2 \sigma^{2}}\right]
$$

where the Gaussian is truncated such that $\theta_{m}$ takes on physical values $\left(\theta_{m}>0\right), \tilde{\mu}$ is the mean value of $\theta_{m}$, and $\sigma$ is the standard deviation of $\theta_{m}$. To take into account systematic uncertainties, each nuisance parameter is convoluted in the statistical probability:

$$
L\left(n \mid \beta, \sum_{k} b_{k}, s_{\mathrm{SM}}, \sum_{m} \theta_{m}\right)=\int \ldots \int \frac{1}{n !} \mu^{n} e^{-\mu} \times \prod_{m} G\left(\theta_{m}>0 \mid \tilde{\mu}, \sigma\right) \mathrm{d} m
$$

where $\mu=\mu\left(\beta, b_{k}, s_{\mathrm{SM}}, \theta_{m}\right)$. The likelihood $L$ is made independent of the nuisance parameters by integrating (5.9) over the nuisance parameter space [89]. This process is computationally very expensive because it requires sampling a nuisance parameter space of large dimensions. Conventional MC integration methods uniformly sample this space and evaluate the integrand at each point. Points with small integrands contribute very little to the likelihood and the peaks of the nuisance-parameter prior distributions $G\left(\theta_{m}>0 \mid \tilde{\mu}, \sigma\right)$ are not sufficiently probed. In this thesis, Markov Chain Monte Carlo (MCMC) techniques [89] are employed for the purpose of properly sampling the peak of the $G$ distributions.

\subsubsection{Markov Chain Integration}

Markov Chain Monte Carlo (MCMC) methods sample the nuisance parameter space employing a Markov chain technique. Following the Metropolis-Hastings algorithm [90], 
the integration starts from a random point and selects the next point using a Gaussian function. The probability densities are then compared: if $p($ new $) / p$ (old) $>1$, the algorithm moves to the next point; if not, the next point is selected with the probability $\Pi$ $=p($ new $) / p($ old $)$. Using this approach, the algorithm spends more time around points which contribute significantly to the integral, while also sampling enough of the parameter space.

The length of the chain is an important parameter of the technique. Long Markov chains are needed to reach a stable solution to the integration. In practice, chain lengths of $1,000,000$ to $10,000,000$ steps are typically used.

\subsubsection{Combination of Multiple Bins and Channels}

The case of a single bin corresponds to a counting experiment. Additional sensitivity is provided when all the bins of a distribution are used to compare the prediction to the data, and the likelihood $L$ is computed for each bin assuming that each bin is independent from the others. The gain in sensitivity arises primarily from different $s / b$ ratios in different bins, e.g. high (low) $s / b$ for high (low) values of the discriminant function. Further sensitivity is achieved by combining the likelihood with that of additional search regions or final states.

Since they can alter the predictions across many bins, shape uncertainties have an important role when multiple bins are considered. Shape uncertainties are conveniently viewed as correlated bin-specific rate uncertainty.

The total likelihood is obtained assuming that all bins are independent, and is given by:

$$
L=\prod_{i} L_{i}
$$

where $L_{i}$ is the likelihood for the $i$ th bin. The case of multiple channels is straightforward: one simply considers the additional bins as if they were from the same distribution. The combination of independent searches relies on the same approach, considering multiple channels from different analyses at the same time. The measurement benefits from the higher statistics and from the correlation of systematic uncertainties among the different channels. When the values of the nuisance parameters are different for different regions, the estimate is performed in each channel independently and then the combination is performed to obtain a higher precision. 


\subsection{Upper Limit and Cross Section Measurement}

Considering the statistical framework described in the previous Section, a likelihood function is used to extract the signal cross section or to set an upper limit, given the data and the backgrounds (with the associated uncertainties). This function is the product of the Poisson probabilities for each bin of each histogram in each channel. These Poisson probabilities are functions of $n_{i}$, the number of observed events in each bin, and $\mu_{i}$, the predictions in each bin; $i$ ranges from 1 to $n_{\text {bins. }}$. The likelihood function is given by:

$$
L=\prod_{i=1}^{n_{\mathrm{bins}}} \frac{\mu_{i}^{n_{i}} e^{-\mu_{i}}}{n_{i} !}
$$

The prediction in each bin is a sum over the signal and background contributions:

$$
\mu_{i}=\sum_{k=1}^{n_{b k g}} b_{i k}+\sum_{k=1}^{n_{s i g}} s_{i k}
$$

where $b_{i k}\left(s_{i k}\right)$ is the background (signal) prediction in the bin $i$ for the process $k$.

Nuisance parameters $\theta_{m}$ affect signal and background predictions. Shape and rate effects due to a given nuisance parameter are treated as $100 \%$ correlated. The overall rate uncertainties do not include the statistical uncertainties, which are taken into account by bin-by-bin uncertainties since they are independent from each other and from any other source of systematic uncertainty. Nuisance parameters $\eta_{i k}$ are associated to bin-by-bin uncertainties in each bin $i$ for each process $k$ and convoluted into the likelihood function. The nuisance parameters are applied starting from shape uncertainties, followed by bin-by-bin uncertainties, and finally overall rate uncertainties [78].

The likelihood is thus a function of the observed data $\mathbf{D}=\left\{n_{i}\right\}$, the signal scale factors $\boldsymbol{\beta}$ (one for each signal process), the nuisance parameters $\boldsymbol{\theta}=\left\{\theta_{s, i k}, \theta_{b, i k}\right\}$ and $\boldsymbol{\eta}=$ $\left\{\eta_{s, i k}, \eta_{b, i k}\right\}$, as well as the central values of the signal $s=\left\{s_{i k}^{0}\right\}$ and background $\boldsymbol{b}=\left\{b_{i k}^{0}\right\}$ predictions, and the the magnitudes of the rate $\boldsymbol{\rho}=\left\{\rho_{b, i k}^{m \pm}, \rho_{s, i k}^{m \pm}\right\}$, shape $\kappa=\left\{\kappa_{b, i k}^{m \pm}, \kappa_{s, i k}^{m \pm}\right\}$, and bin-by-bin $\boldsymbol{\delta}=\left\{\delta_{b, i k^{\prime}}^{0} \delta_{b, i k}^{m \pm}, \delta_{s, i k^{\prime}}^{0} \delta_{s, i k}^{m \pm}\right\}$ uncertainties:

$$
L=L(\mathbf{D} \mid \boldsymbol{\beta}, \boldsymbol{\eta}, \boldsymbol{s}, \boldsymbol{b}, \boldsymbol{\rho}, \boldsymbol{\kappa}, \boldsymbol{\delta})
$$

We use the MCLIMIT software package [91] to numerically integrate $L$ over the nuisance parameters:

$$
L^{\prime}(\beta)=\int L(\mathbf{D} \mid \beta, \boldsymbol{\theta}, \boldsymbol{\eta}, \boldsymbol{s}, \boldsymbol{b}, \boldsymbol{\rho}, \boldsymbol{\kappa}, \boldsymbol{\delta}) \pi(\boldsymbol{\theta}) \pi(\boldsymbol{\eta}) d \boldsymbol{\theta} d \boldsymbol{\eta}
$$


where the $\pi$ functions are the Bayesian priors assigned to each nuisance parameter. The measured cross section corresponds to the maximum of $L^{\prime}$, which occurs at $\beta^{\text {max. }}$

$$
\sigma_{\text {meas }}=\beta^{\max } \sigma_{\mathrm{SM}}
$$

The asymmetric uncertainties quoted correspond to the shortest interval containing $68 \%$ of the integral of the posterior, assuming a uniform prior in $\beta, \pi(\beta)=1$ :

$$
0.68=\frac{\int_{\beta_{\text {low }}}^{\beta_{\text {high }}} L^{\prime}(\beta) \pi(\beta) d \beta}{\int_{0}^{\infty} L^{\prime}(\beta) \pi(\beta) d \beta}
$$

The same posterior is used to determine the 95\% C.L. upper limit $l$ satisfying:

$$
0.95=\frac{\int_{0}^{l} L^{\prime}(\beta) \pi(\beta) d \beta}{\int_{0}^{\infty} L^{\prime}(\beta) \pi(\beta) d \beta}
$$

\subsubsection{Expected and Observed Limits and Cross Sections}

Before using data to set upper limits or measure the signal production cross section, a consistency-check on simulated data is performed, in order to test the machinery and to ensure that no biases are introduced in the analysis.

A pseudo-experiment is simulated by generating pseudo-data for each bin from a Poisson probability density function. A value is assigned to each source of uncertainty by varying the nuisance parameters drawn from prior distributions. The effect is then incorporated into the predictions that are combined to provide a simulated data distribution. In the case of upper limits, pseudo-data are generated without including signal. Upper limits and cross sections are then derived as previously described.

The median expected value is the median of the distribution of upper limits or cross sections from pseudo-experiments. The expected uncertainty on the limits or cross sections corresponds to the boundary of a $68 \%$ interval of this distribution. Sufficient pseudoexperiments have to be generated to ensure sufficient statistical accuracy; typically $\mathcal{O}(10000)$ experiments are generated.

\subsection{Significance Calculation}

In addition to measuring cross sections or setting limits, it is important to establish evidence or observation of a given process. The $p$-value, which is the probability of observing a signal that is as large as the one observed or larger, assuming that the signal 
is absent, is usually calculated to evaluate the significance of the signal observation. By convention, an observed $p$-value of less than $1.35 \times 10^{-3}$ constitutes evidence for a signal, and an observed $p$-value less than $2.87 \times 10^{-7}$ constitutes a discovery. These correspond to $+3 \sigma$ and $+5 \sigma$ excesses, respectively, where the correspondence between the $p$-value and the number of $\sigma$ is computed using the integral of one side of a Gaussian distribution.

Usually, an ensemble-based calculation is performed. Pseudo-experiments are generated, simulating signal and background processes mixed together and randomly fluctuated according to the distribution of the uncertainties. Each pseudo-experiment passes through the same machinery as the data, yielding one outcome. The experimental outcomes are ranked on a one-dimensional scale as more or less signal- or background-like based on a quantity known as a test statistic. In this thesis, both $\beta^{\max }=\sigma_{\text {meas }} / \sigma_{\mathrm{SM}}$ and the loglikelihood ratio:

$$
-2 \ln Q=-2 \ln \frac{L\left(\mathbf{D} \mid \boldsymbol{\beta}, \hat{\boldsymbol{\theta}}, \hat{\boldsymbol{\eta}}, \boldsymbol{s}=\boldsymbol{s}^{\mathrm{SM}}, \boldsymbol{b}, \boldsymbol{\rho}, \boldsymbol{\kappa}, \boldsymbol{\delta}\right)}{L(\mathbf{D} \mid \boldsymbol{\beta}, \hat{\boldsymbol{\theta}}, \hat{\boldsymbol{\eta}}, \boldsymbol{s}=0, \boldsymbol{b}, \boldsymbol{\rho}, \boldsymbol{\kappa}, \boldsymbol{\delta})}
$$

are employed as test statistics. In (5.18), $\hat{\theta}$ and $\hat{\eta}$ are the best-fit values of the nuisance parameters which maximize $L$ given the data $D$, assuming the presence of signal with the predicted rate, and $\hat{\hat{\theta}}$ and $\hat{\eta}$ are the best-fit values of the nuisance parameters which maximize $L$ assuming no signal.

Distributions of $\beta^{\max }$ or of $-2 \ln Q$ are derived from the results of simulated pseudoexperiments, which are generated assuming (test hypothesis) or neglecting (null hypothesis) the signal. The integral of the null hypothesis distribution from the median value of the test hypothesis to infinitely large positive values gives the expected $p$-value. The observed $p$-value is estimated computing the same integral from the $\beta^{\max }$ or $-2 \ln Q$ value obtained using data. Many pseudo-experiments are required in order to achieve sufficient precision on the significance estimate. When the sensitivity of an analysis to the signal is high, the generation of such a large sample of pseudo-experiments becomes computationally very expensive. In this case, an asymptotic approximation is applied by generating a smaller number of pseudo-experiments and fitting the $-2 \ln Q$ distribution with a Gaussian function [92]. 


\section{Chapter 6}

\section{Observation of $s$-Channel Single Top Quark Production}

The study of single top quark production is particularly interesting because of the direct dependence of the cross section on the magnitude of the $W t b$ coupling. Furthermore $s-$ channel single top quark production is of interest since significant deviations from the SM expectation would indicate the presence of contributions from non-SM particles such as higher mass partners of the $W$ boson (denoted as $W^{\prime}$-like resonances) or charged Higgs bosons. Single top quark production was observed at the Tevatron in 2009 [68, 93, 94]. However, $s$-channel production was not observed independently before the work presented in this thesis. Observation of single top quark production in the $t$-channel was first obtained at the Tevatron by the D0 experiment [95], and was also established by ATLAS and CMS $[96,97]$. The s-channel contribution could not be firmly isolated at the LHC because it has an unfavorable production rate compared to the background rates. The D0 Collaboration reported the first evidence of s-channel single top quark production in 2013 [98], measuring a cross section of $1.10_{-0.31}^{+0.33}$ (stat+syst) $\mathrm{pb}$ and quoting a significance of 3.7 standard deviations. More recently, CDF obtained 3.8 standard deviation evidence using events containing one isolated muon or electron, large $\mathbb{E}_{T}$, and two jets, at least one of which is $b$-tagged [99]. This sample will be referred in the following as the " $\ell v b \bar{b}$ " sample. To add acceptance to this data set, the analysis presented here uses for the first time events with large $\mathbb{E}_{T}$, two or three jets of which one or more are $b$-tagged, and no detected electron or muon candidates. This sample of events is referred to as the " $E_{T} b \bar{b}$ " sample and contains s-channel single top quark contributions where $t \rightarrow W b \rightarrow \ell v$ and $\ell$ is an electron or a muon that is not identified in the detector, or a $\tau$ that decays hadronically. 


\subsection{Search for s-Channel Single Top Quark Production in the $\boldsymbol{E}_{T} b \bar{b}$ Sample}

In this Section, the search for s-channel single top quark production in the $\mathbb{E}_{T} b \bar{b}$ sample is reported. Most of the techniques developed for the low-mass Higgs boson search [100] in the same data sample are exploited, including the HOBIT $b$-tagger [63]. By combining the results of the searches in this and in the $\ell v b \bar{b}$ sample, an improved sensitivity to $s$-channel single top production from the full CDF II data set is obtained.

\subsubsection{Definition of the Analysis Subsamples}

In addition to the event preselection that has been introduced in Section 4.2.2, the two jets with the largest transverse energies, $E_{T}^{j_{1}}$ and $E_{T}^{j_{2}}$, are required to satisfy $25<E_{T}^{j_{1}}<200$ $\mathrm{GeV}$ and $20<E_{T}^{j_{2}}<120 \mathrm{GeV}$. The sample with no additional jets is the two-jet sample; a three-jet sample is added where the third jet has $15<E_{T}^{j_{3}}<100 \mathrm{GeV}$.

The limits on the maximum energy of the jets are applied since the HOBIT algorithm overestimates the tagging efficiency for the very-high-energy jets. Figure 6.1 shows the HOBIT tagging efficiency and the mistag rate for jets with large $E_{T}$. Above $E_{T}=200 \mathrm{GeV}$, the tag rate rises for both $b$ jets and light-flavor jets, reaching $100 \%$ at about $250 \mathrm{GeV}$ for the HOBIT loose operating point. In general, the $b$-tag efficiency increases with increasing jet $E_{T}$ due to the greater displacement of $B$ hadrons. Similarly, the mistag efficiency increases, due to the higher rapidity and $p_{T}$ of tracks in high- $E_{T}$ jets. Since the HOBIT algorithm was trained on MC samples with few jets at high energy, it is insensitive to kinematic differences between high- $E_{T} b$ jets and high- $E_{T}$ light-flavour jets and it tags all the jets above a certain $E_{T}$ threshold independently from the flavour of the originating parton.

Preselected events are assigned to three independent subsamples depending on the HOBIT output of the two leading jets. As described in Section 3.2.3, jets with HOBIT values larger than 0.98 are defined as tightly tagged (" $\mathrm{T}$ "), whereas jets with outputs between 0.72 and 0.98 are defined as loosely tagged ("L"). The two-jet and three-jet samples are analyzed separately, leading to six event subsamples with different signal to background ratios. This strategy improves sensitivity and helps separate s-channel single top quark production, enhanced in the double-tag categories, from the $t$-channel production, enhanced in the single-tag categories. 

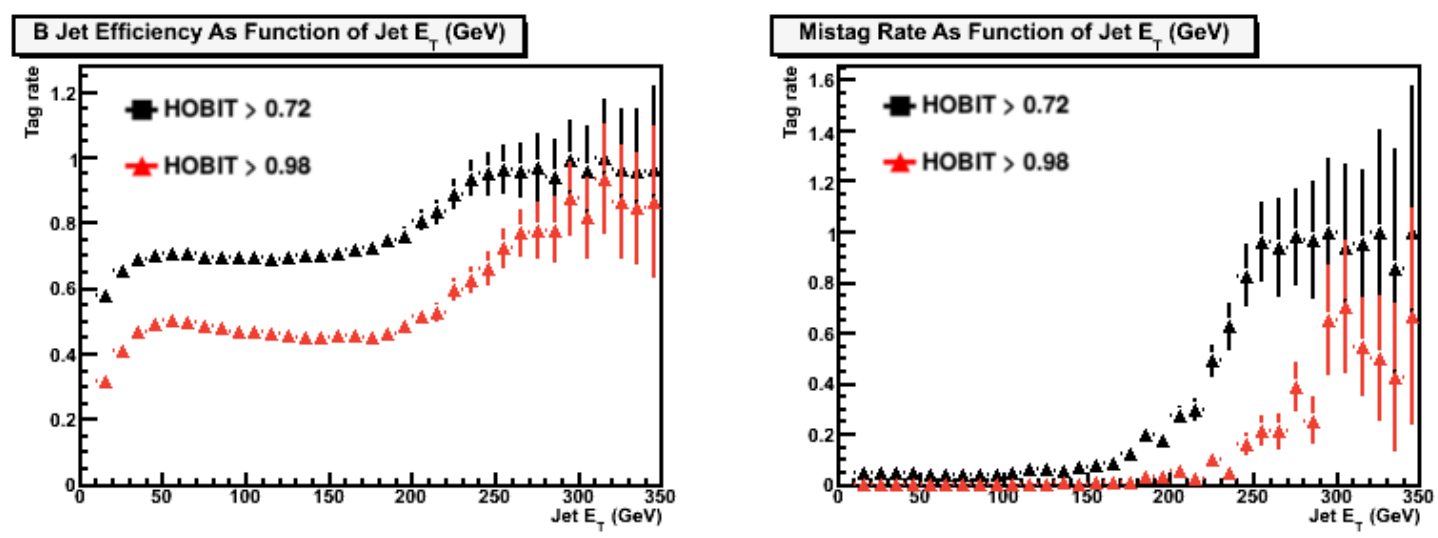

Figure 6.1 - The $b$-tag efficiency and the mistag rate as a function of jet $E_{T}$ in MC samples independent from the samples used in the training. The black triangles are for the HOBIT loose operating point and the colored triangles are for the HOBIT tight operating point. The $\mathrm{x}$-axis is extended compare to Figure 3.6 to show the behaviour of the $b$-tag efficiency and the mistag rate for high- $E_{T}$ jets. From [101]

\subsubsection{Multivariate Discriminants}

In order to better separate the signal from the backgrounds, a staged neural network (NN) technique is employed. A first network, $\mathrm{NN}_{\mathrm{QCD}}$, is trained to discriminate QCD multijet events from signal events. Events that satisfy a minimal requirement on the $\mathrm{NN}_{\mathrm{QCD}}$ output variable are further analyzed by a function, $\mathrm{NN}_{\text {sig }}$, derived from the outputs of two additional neural networks, $\mathrm{NN}_{V \text { jets }}$ and $\mathrm{NN}_{t \bar{t}}$, designed respectively to separate the signal from $V+$ jets (and some more QCD multijet events) and $t \bar{t}$ backgrounds.

The $\mathrm{NN}_{\mathrm{QCD}}$ discriminant is trained using QCD multijet data events as the background sample. Since the kinematic properties associated with the presence of a $W$ boson in the $s$-channel single top quark and $W+$ jets production processes are very similar, in contrast with those of events originating from QCD multijet production, $W+$ jets events are used as signal sample. The discriminant is trained separately for the two-jet and three-jet samples using twelve to fifteen activity-derived $\left(\mathbb{E}_{T}, \not_{T}[102]\right)$, angular $\left(\Delta \varphi\left(\vec{E}_{T}, \vec{p}_{T}\right)\right.$, angular separations between $\vec{E}_{T}, \vec{p}_{T}$ and jet directions), and event-shape (sphericity ${ }^{1}$ ) observables for the input variables. By requiring a suitable threshold on $\mathrm{NN}_{\mathrm{QCD}}$, the QCD multijet contribution is reduced by $88 \%$ while keeping $85 \%$ of the signal. The observed and estimated event yields after the $\mathrm{NN}_{\mathrm{QCD}}$ requirement are shown in Tables 6.1 and 6.2.

The two additional networks, $\mathrm{NN}_{V \text { jets }}$ and $\mathrm{NN}_{t \bar{t}}$, are trained for events that satisfy the minimum requirement on the $\mathrm{NN}_{\mathrm{QCD}}$ output variable. The first, $\mathrm{NN}_{V \text { jets }}$ is trained to separate the s-channel single top quark signal from $V+$ jets and the remaining QCD multijet backgrounds. A simulated signal is used in the training, while the background sample

\footnotetext{
${ }^{1}$ The event sphericity is defined by $S=\frac{3}{2}\left(\lambda_{-} 2+\lambda_{-} 3\right)$, where the sphericity tensor is $S^{\alpha \beta}=\left(\sum_{-} i p_{-} i^{\alpha} p_{\_}{ }^{\beta}\right) /\left(\sum_{-} i p_{-} i^{2}\right)$ and $\lambda \_1>\lambda \_2>\lambda \_3$ are its three eigenvalues and satisfy $\lambda \_1+\lambda \_2+\lambda \_3=$ 1. The index $i$ refers to each jet in the event.
} 
consists of pretag data events that satisfy the requirement on $\mathrm{NN}_{\mathrm{QCD}}$, reweighted by the tag-rate probability as derived from the TRM (see Section 4.3.3). The $\mathrm{NN}_{\mathrm{QCD}}$ requirement changes the pretag data composition, enhancing the $V+$ jets contribution and selecting QCD multijet events with properties closer to those expected for $V+$ jets events. The contribution from other processes to pretag events is negligible and the background model obtained by reweighting these events via the tag-rate probability accounts for both the $V+$ jets and QCD multijet event contributions, allowing for a more straightforward training of the $\mathrm{NN}_{V \text { jets. }}$. The second, $\mathrm{NN}_{t \bar{t}}$, is trained to separate $s$-channel single top quark from $t \bar{t}$ production using simulation for both components. Variables which describe the energy and momentum flow in the detector and angular variables are used in the training of the $\mathrm{NN}_{V \text { jets }}$ and $\mathrm{NN}_{t \bar{t}}$ discriminants. Some of the most separating variables used in the training of the $\mathrm{NN}_{V \text { jets }}$ and $\mathrm{NN}_{t \bar{t}}$ discriminants, as well as the ones used in the training of the $\mathrm{NN}_{\mathrm{QCD}}$, are shown in Figures 6.2 and 6.3:
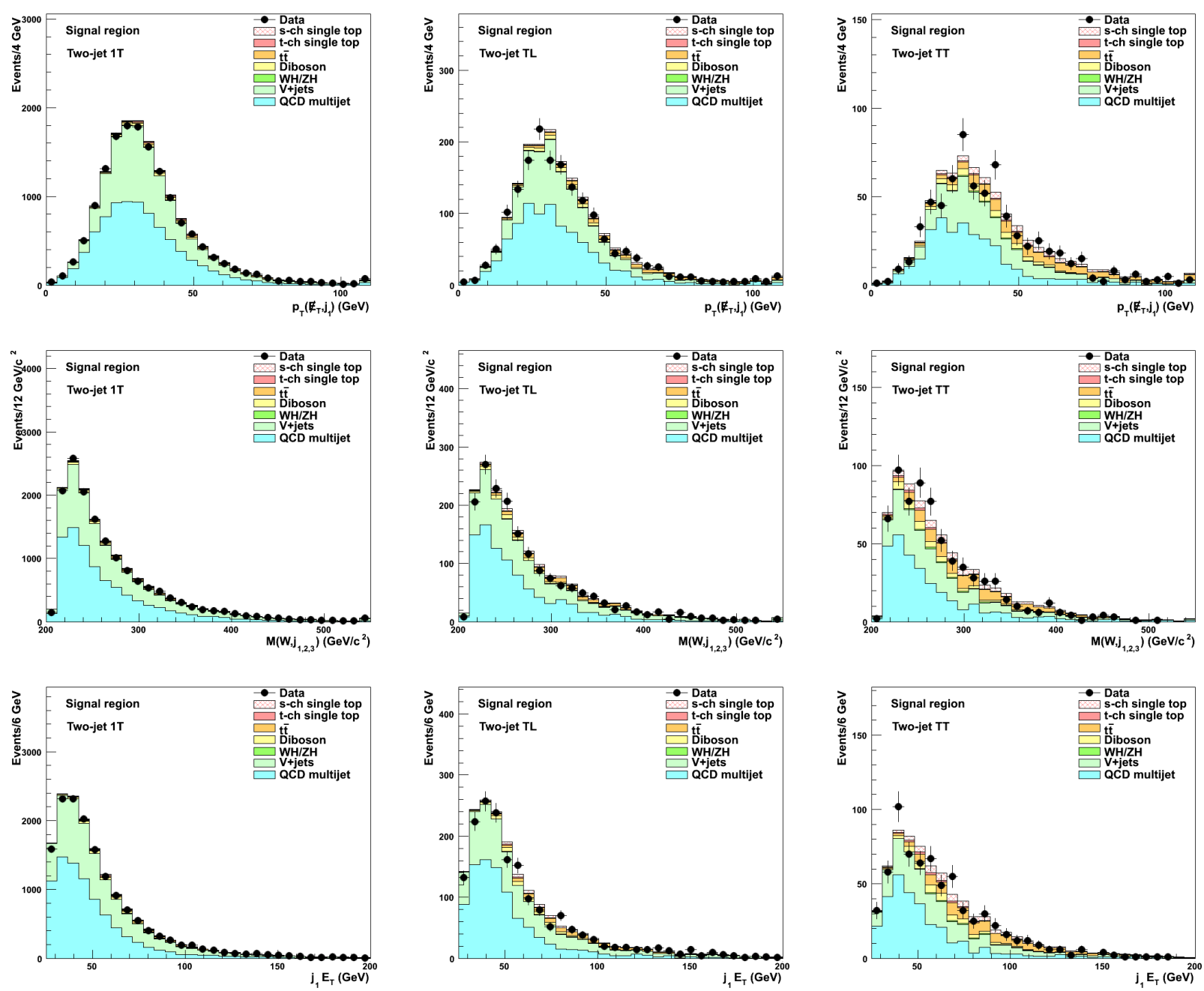

Figure 6.2 - Predicted and observed distributions in the signal region for some of the most separating kinematic variables used in the training of the $\mathrm{NN}_{\mathrm{QCD}}, \mathrm{NN}_{V j \mathrm{jets}}$ and $\mathrm{NN}_{t \bar{t}}$ discriminants in the two-jet subsample.

The final discriminant, $\mathrm{NN}_{\text {sig }}$, is defined as the quadrature sum of the $\mathrm{NN}_{V \text { jets }}$ and $\mathrm{NN}_{t \bar{t}}$ 

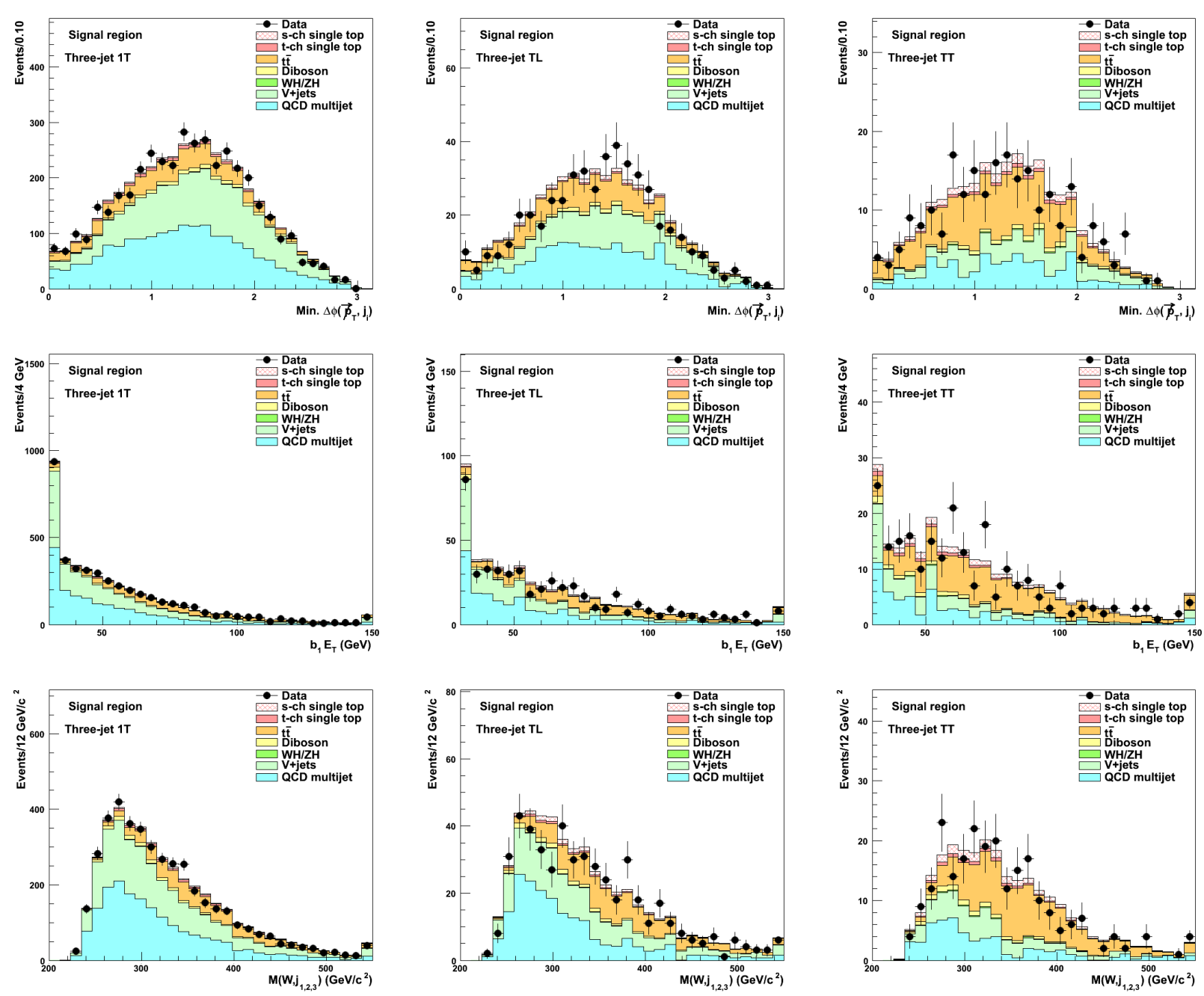

Figure 6.3 - Predicted and observed distributions in the signal region for some of the most separating kinematic variables used in the training of the $\mathrm{NN}_{\mathrm{QCD}}, \mathrm{NN}_{V \text { jets }}$ and $\mathrm{NN}_{t \bar{t}}$ discriminants in the three-jet subsample.

output variables, both weighted by an appropriate weight optimized to improve the sensitivity $^{2}$ in each analysis subsample, taking into account the different background contributions. Figure 6.4 shows the predicted and observed shapes of the $\mathrm{NN}_{\text {sig }}$ output variable for each of the six event subsamples.

The modeling of SM backgrounds is tested in several control samples. A first EWK control sample, defined in Section 4.2.2, is independent from the signal sample and is sensitive primarily to top-quark pair, $V+$ jets, and, to a lesser extent, $V V$ production. A second QCD control sample is defined to contain events that do not meet the minimal requirement on the $\mathrm{NN}_{\mathrm{QCD}}$ output variable but otherwise satisfy the preselection criteria. This event sample, dominated by QCD multijet production, is used to validate the data-driven QCD multijet background model and to obtain scale factors, ranging from 0.7 to 0.9 , for

${ }^{2}$ The sensitivity is estimated using the binned significance as figure of merit. The binned significance is defined as the quadrature sum of the signal-over-background ratios calculated bin-by-bin over the bins of the $\mathrm{NN}_{\text {sig }}$ output value. The effect of the systematic uncertainties is not taken into account in this calculation. 
Table 6.1 - Numbers of predicted and observed two-jet events in the 1T, TL, and TT subsamples. The uncertainties on the predicted numbers of events are due to the theoretical-crosssection uncertainties and the uncertainties on signal and background modeling. Central values and uncertainties are obtained from the fit to the data which incorporates the theoretical constraints.

\begin{tabular}{cccc}
\hline \hline Category & $1 \mathrm{~T}$ & $\mathrm{TL}$ & $\mathrm{TT}$ \\
\hline$t$-ch single top & $161 \pm 31$ & $10.8 \pm 2.1$ & $9.2 \pm 1.7$ \\
$t \bar{t}$ & $243 \pm 24$ & $84.8 \pm 9.3$ & $92.4 \pm 8.4$ \\
Diboson & $285 \pm 26$ & $51.3 \pm 4.6$ & $37.2 \pm 3.4$ \\
$V H$ & $12.6 \pm 1.4$ & $6.6 \pm 0.8$ & $7.6 \pm 0.8$ \\
$V+$ jets & $6528 \pm 2048$ & $694 \pm 216$ & $220 \pm 69$ \\
MJ & $8322 \pm 180$ & $928 \pm 59$ & $300 \pm 32$ \\
\hline Signal & $86.2 \pm 47.7$ & $41.8 \pm 23.2$ & $45.9 \pm 25.3$ \\
\hline Total prediction & $15557 \pm 2056$ & $1733 \pm 224$ & $663 \pm 76$ \\
\hline Observed & 15312 & 1743 & 686 \\
\hline \hline
\end{tabular}

Table 6.2 - Numbers of predicted and observed three-jet events in the 1T, TL, and TT subsamples.

\begin{tabular}{cccc}
\hline \hline Category & $1 \mathrm{~T}$ & $\mathrm{TL}$ & $\mathrm{TT}$ \\
\hline$t$-ch single top & $82.2 \pm 15.8$ & $7.5 \pm 1.5$ & $6.8 \pm 1.3$ \\
$t \bar{t}$ & $597 \pm 60$ & $118 \pm 13$ & $110 \pm 10$ \\
Diboson & $108 \pm 10$ & $15.7 \pm 1.5$ & $8.8 \pm 0.8$ \\
$V H$ & $6.0 \pm 0.7$ & $1.9 \pm 0.2$ & $2.2 \pm 0.2$ \\
$V+$ jets & $1610 \pm 505$ & $165 \pm 51$ & $50 \pm 16$ \\
MJ & $1818 \pm 49$ & $188 \pm 15$ & $55.9 \pm 7.6$ \\
\hline Signal & $45.7 \pm 25.3$ & $15.4 \pm 8.5$ & $16.2 \pm 8.9$ \\
\hline Total prediction & $4220 \pm 511$ & $495 \pm 55$ & $234 \pm 20$ \\
\hline Observed & 4198 & 490 & 237 \\
\hline \hline
\end{tabular}

normalizing modeled QCD multijet contributions to the TT, TL, and 1T event subsamples. Figure 6.5 illustrates the technique used to derive the QCD multijet normalization.

Comparisons of modeled and observed distributions for multiple kinematic variables, including those used as inputs to the $\mathrm{NN}_{\mathrm{QCD}}, \mathrm{NN}_{V \text { jets }}$, and $\mathrm{NN}_{t \bar{t}}$, are used to validate the accuracy of the model and are presented in the Appendix B.

\subsubsection{Cross Section Extraction and Significance Calculation}

Several sources of systematic uncertainty are taken into account. Uncertainties on theoretical cross section values are included for $t \bar{t}(3.5 \%), t$-channel single top quark $(6.2 \%)$, $V V(6 \%), V H(5 \%)$, and $W+c(23 \%)$ processes [16, 22, 79, 80,77]. Other systematic uncertainties arise from the normalization of the $V$-plus-heavy-flavour (30\%) and of the QCD 

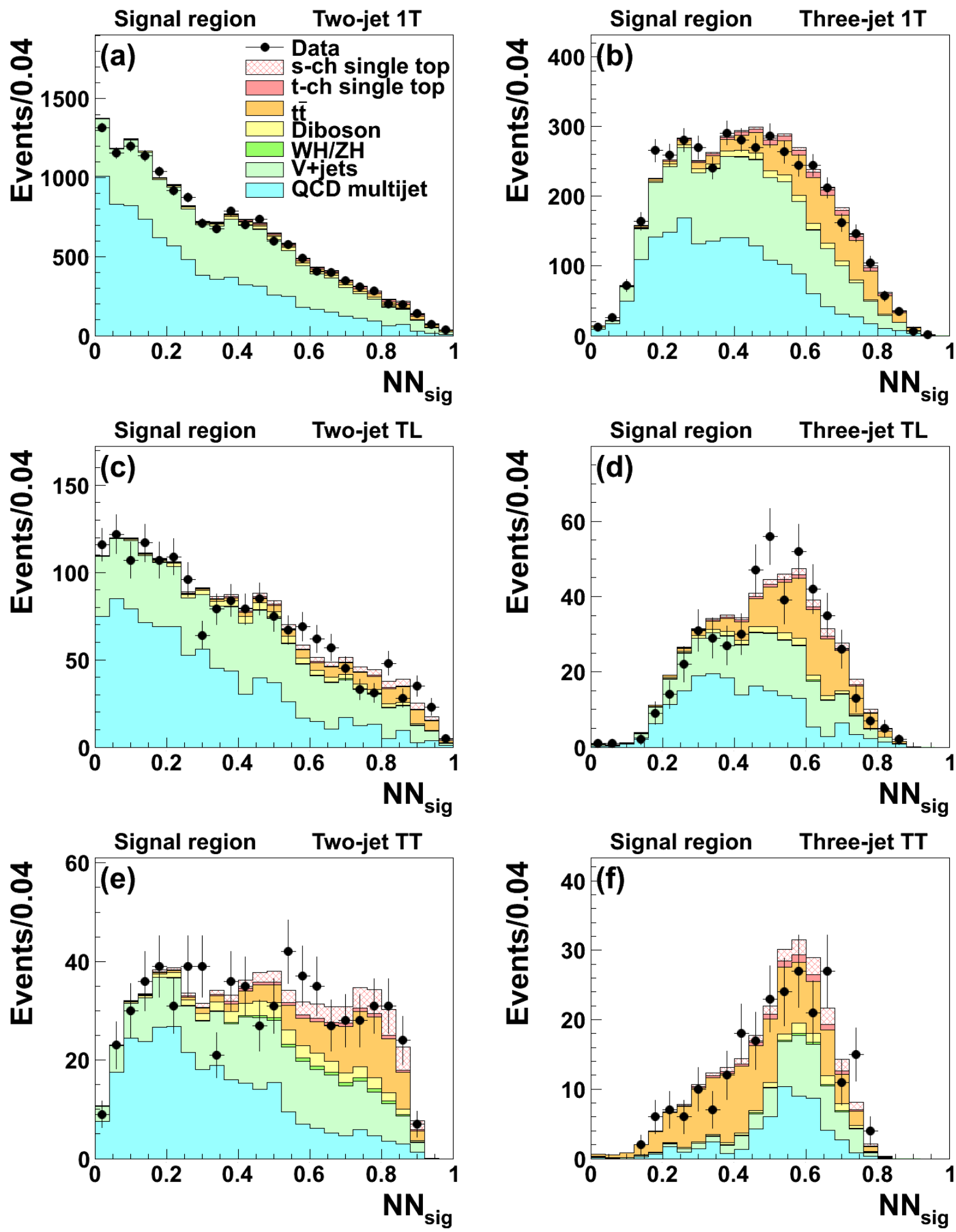

Figure 6.4 - Predicted and observed final discriminant distributions in the signal region, for (a) 1T two-jet, (b) 1T three-jet, (c) TL two-jet, (d) TL three-jet, (e) TT two-jet and (f) TT three-jet event subsamples.

multijet $(3 \%-7 \%)$ background contributions. All samples whose normalizations are not constrained according to the data are subject to a luminosity uncertainty of $6 \%$ [70]. Fur- 


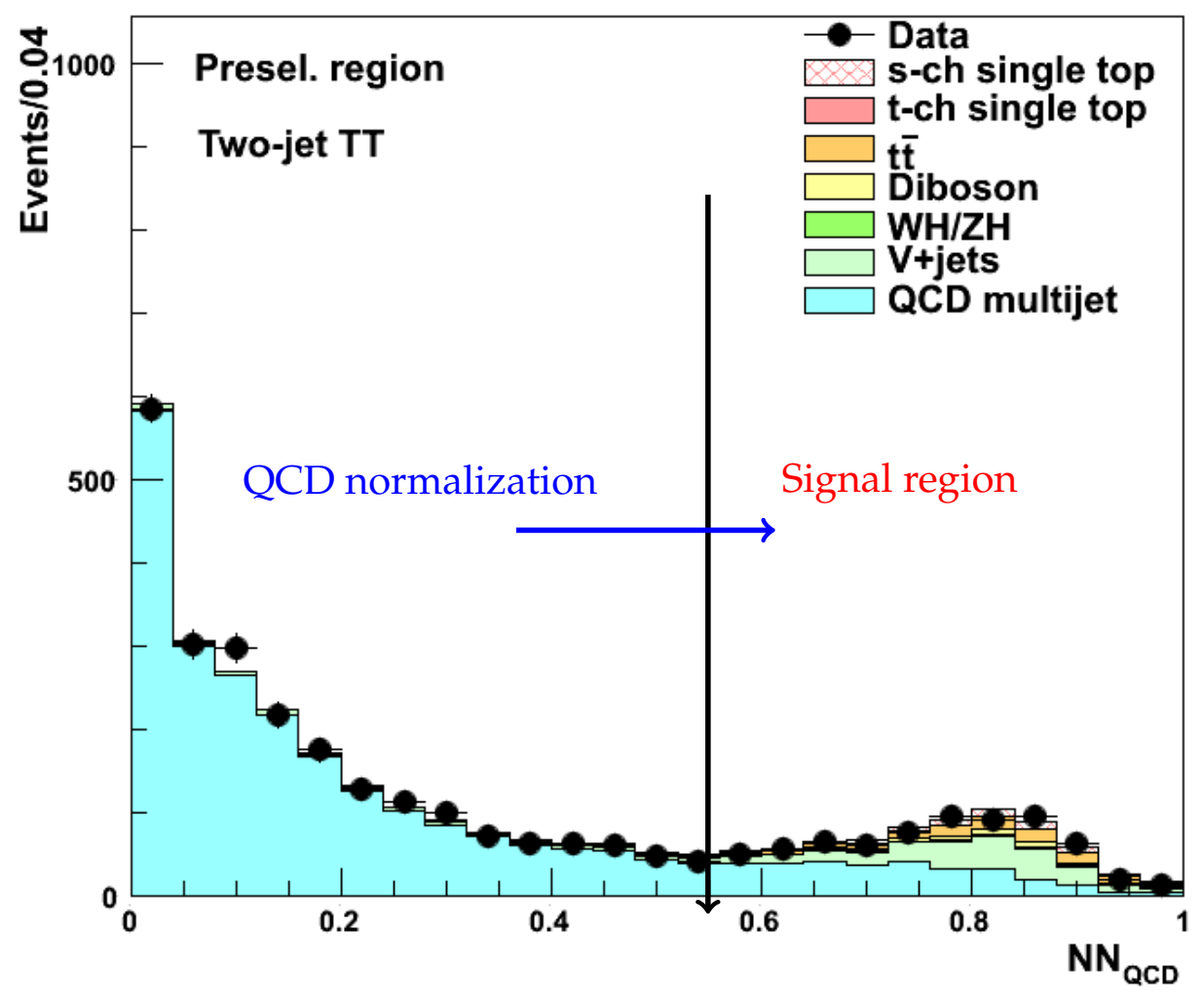

Figure 6.5 - The $\mathrm{NN}_{\mathrm{QCD}}$ output variable in the preselection region for the TT two-jet subsample. The strategy applied to derive the QCD multijet normalization is summarized. The subsample composed by the events that do not satisfy the $\mathrm{NN}_{\mathrm{QCD}}>0.55$ requirement is used to calculate a scale factor, as described in Section 4.3.3. The scale factor is applied to the events in the signal region, defined by the the $\mathrm{NN}_{\mathrm{QCD}}>0.55$ requirement. 
thermore, uncertainties are assigned due to the efficiencies of the lepton antiselection criteria (2\%). A normalization uncertainty of $2 \%$ due to variations in the assumed parton distribution functions is also assigned. To account for differences in the trigger efficiency in data and simulation, a 1 to $3 \%$ rate uncertainty is assigned.

Possible mismodeling in the HOBIT $b$-tagging efficiency is taken into account by applying scale factors to the simulation to correct its $b$-tagging efficiency to that of data. Uncertainties on the HOBIT scale factor determination are included, ranging from $8 \%$ to $16 \%$ [63]. Mistag rate uncertainties $(20 \%-30 \%$ ) are also included (see Section 4.3.2).

Uncertainties in the jet energy scale [56] are included by correlating the variations in the predicted yields of signals and backgrounds (of the order of $1 \%-6 \%$ ) with the corresponding distortions in the predicted kinematic distributions arising from jet energy scale shifts in all samples except the QCD multijet background, which is determined entirely from data. An additional systematic uncertainty is incorporated for the QCD multijet model, accounting for shape variations in the QCD multijet prediction, obtained by varying the tag-rate probabilities by one standard deviation from their central values.

The dominant systematic uncertainties arise from the $V+\mathrm{HF}$ normalization, $b$-tagging efficiencies and mistag rates, and the shape of the data-driven QCD multijet model. As already mentioned in Chapter 5 , the separating power of the $\mathrm{NN}_{\text {sig }}$ discriminant helps in constraining both rate and shape uncertainties. As an example, the $V+\mathrm{HF}$ normalization systematic uncertainty gets constrained to a post-fit value of $12 \%$ in the single-tag sample and a $21 \%$ in the double-tag samples, compared to a pre-fit value of $30 \%$.

A combined likelihood is formed, which is the product of Poisson probabilities for each bin of the six $\mathrm{NN}_{\text {sig }}$ discriminants shown in Figure 6.4, as described in Section 5.2. To account for systematic uncertainties, a Bayesian technique is used, in which each independent source of systematic uncertainty is assigned a nuisance parameter with a Gaussian prior probability density, truncated when necessary to ensure non-negative event yields. The impact of each nuisance parameter is propagated to the predictions of the signal and background yields in each bin of each histogram in the analysis. A non-negative uniform prior probability distribution is assumed for the s-channel single top quark cross section, which is extracted from its posterior probability density after integrating over all nuisance parameters. Following the procedure shown in Section 5.1.2, results from each of the six search subsamples are combined by taking the product of their likelihoods and simultaneously varying the correlated uncertainties.

The measured s-channel single top quark cross section in the $\mathbb{E}_{T} b \bar{b}$ sample is $1.12_{-0.57}^{+0.61}$ (stat+syst) $\mathrm{pb}$. The extracted posterior probability distribution for $\sigma_{s}$ is presented in Figure 6.6.

The $p$-value is determined using an ensemble-based calculation to be $3.1 \times 10^{-2}$, corresponding to a significance of 1.9 standard deviations. The median expected significance assuming that a signal is present at the SM rate is 1.8 standard deviations. The distribu- 


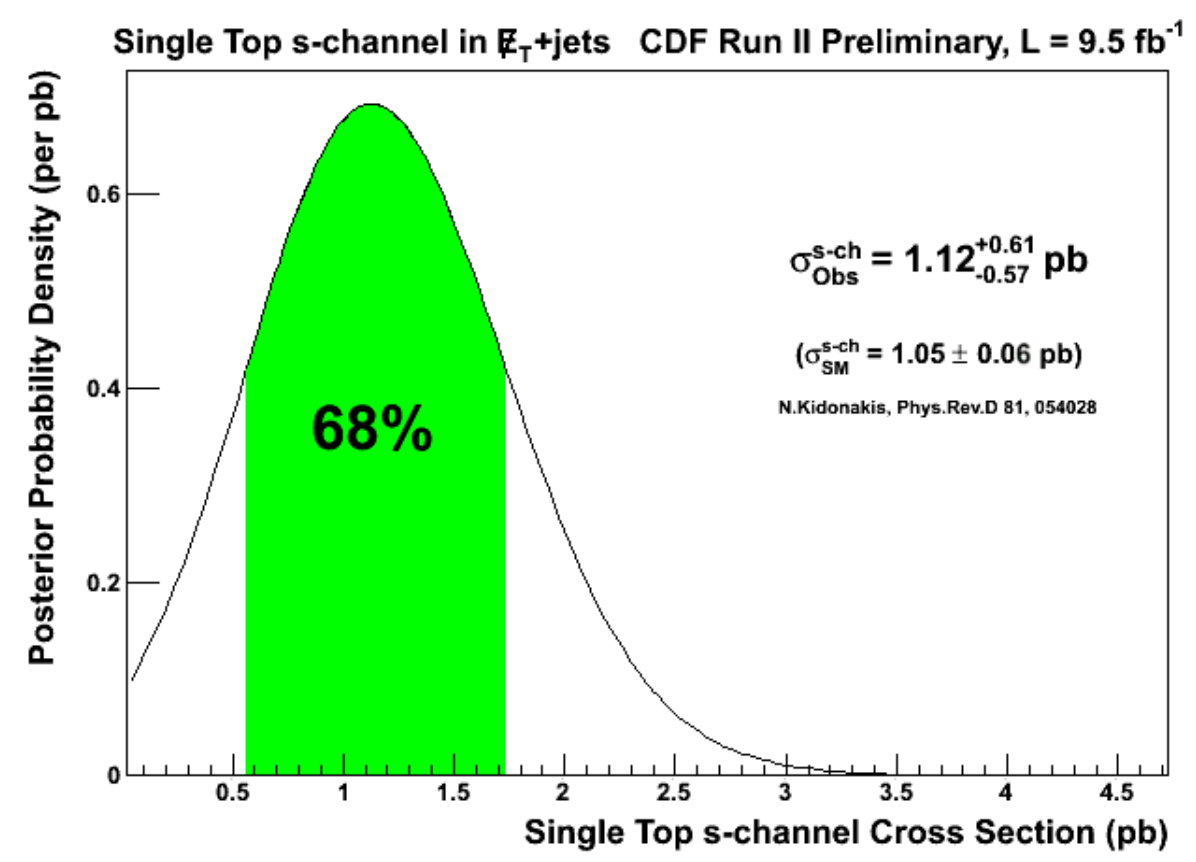

Figure 6.6 - The posterior probability distribution for the CDF $\mathbb{E}_{T} b \bar{b}$ analysis.

tions of pseudo-experiments that include background-only, or signal-plus-background, contributions are presented in Figure 6.7.

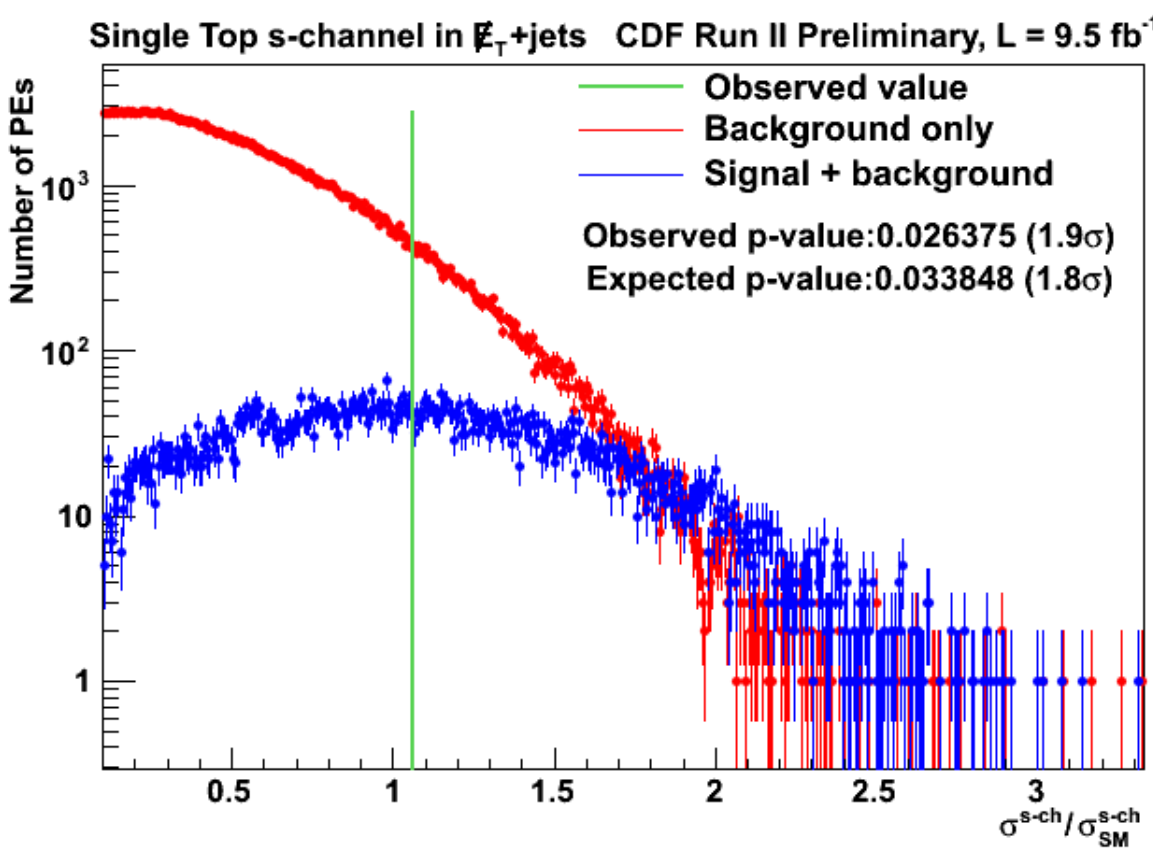

Figure 6.7 - Pseudo-experiment distributions for the background-only (red) and SM-signalplus-background (blue) hypotheses from the CDF $\mathbb{E}_{T} b \bar{b}$ analysis. 


\subsection{CDF Combination}

The result of the $E_{T} b \bar{b}$ analysis is combined with the result of a similar search in the $\ell v b \bar{b}$ sample [99]. In that search, candidate events were selected by requiring exactly one reconstructed muon or electron in the final state. Hence, no such events are included in the $\mathbb{E}_{T} b \bar{b}$ analysis described above. Four independent tagging categories, according to the score of the HOBIT tagger on the two leading jets (tight-tight TT, tight-loose TL, singletight $1 \mathrm{~T}$, and loose-loose LL), were analyzed separately. Events were also divided into three independent samples based on different categories of reconstructed leptons. To discriminate the signal from backgrounds, neural networks were employed. These neural networks were optimized separately for each tagging and lepton category. Correlated systematic uncertainties were treated as described above for the $\mathbb{E}_{T} b \bar{b}$ search. Finally, a binned-likelihood technique was applied to the final NN output to extract the $s$-channel single top quark cross section. The significance of the result from the $\ell v b \bar{b}$ channel was 3.8 standard deviations, and the measured cross section was $1.41_{-0.42}^{+0.44}$ (stat+syst) pb, assuming a top-quark mass of $172.5 \mathrm{GeV} / \mathrm{c}^{2}$.

The two analyses are combined by taking the product of their likelihoods and simultaneously varying the correlated uncertainties, following the same procedure explained in Section 5.1.2. The uncertainties associated with the theoretical cross sections of the $t \bar{t}, t-$ channel electroweak single top quark, $V V$, and $V H$ production processes; the luminosity; the $b$-tagging efficiency; and the mistag rate are considered fully correlated between the two searches. The combined measurement results in an s-channel single top quark production cross section of $1.36_{-0.32}^{+0.37} \mathrm{pb}$, consistent with the $\mathrm{SM}$ cross section of $1.05 \pm 0.05$ $\mathrm{pb}$ [22]. The extracted posterior probability distribution for $\sigma_{s}$ is presented in Figure 6.8.

The combined background-only $p$-value is $1.6 \times 10^{-5}$, which corresponds to a signal significance of 4.2 standard deviations. The median expected significance is 3.4 standard deviations. The distributions of pseudo-experiments that include background-only, or signal-plus-background, contributions are presented in Figure 6.9.

\subsection{Tevatron Combination}

The CDF result is then combined with the result of the analysis performed by the D0 Collaboration [98], that established the first evidence for s-channel single top quark production. D0 analyzed a final-state topology that contains single top quark events in which the $W$ boson from the decay of the top quark decays leptonically producing an electron or a muon and an associated neutrino. It selected events that contain only one isolated lepton $\ell$ with large transverse momentum $p_{T}$, large imbalance $\mathbb{E}_{T}$, two or three jets with large $p_{T}$, and one or two $b$ jets. To identify $b$ jets, multivariate techniques are used [103]. Additional selection criteria were applied to exclude kinematic regions that are difficult 


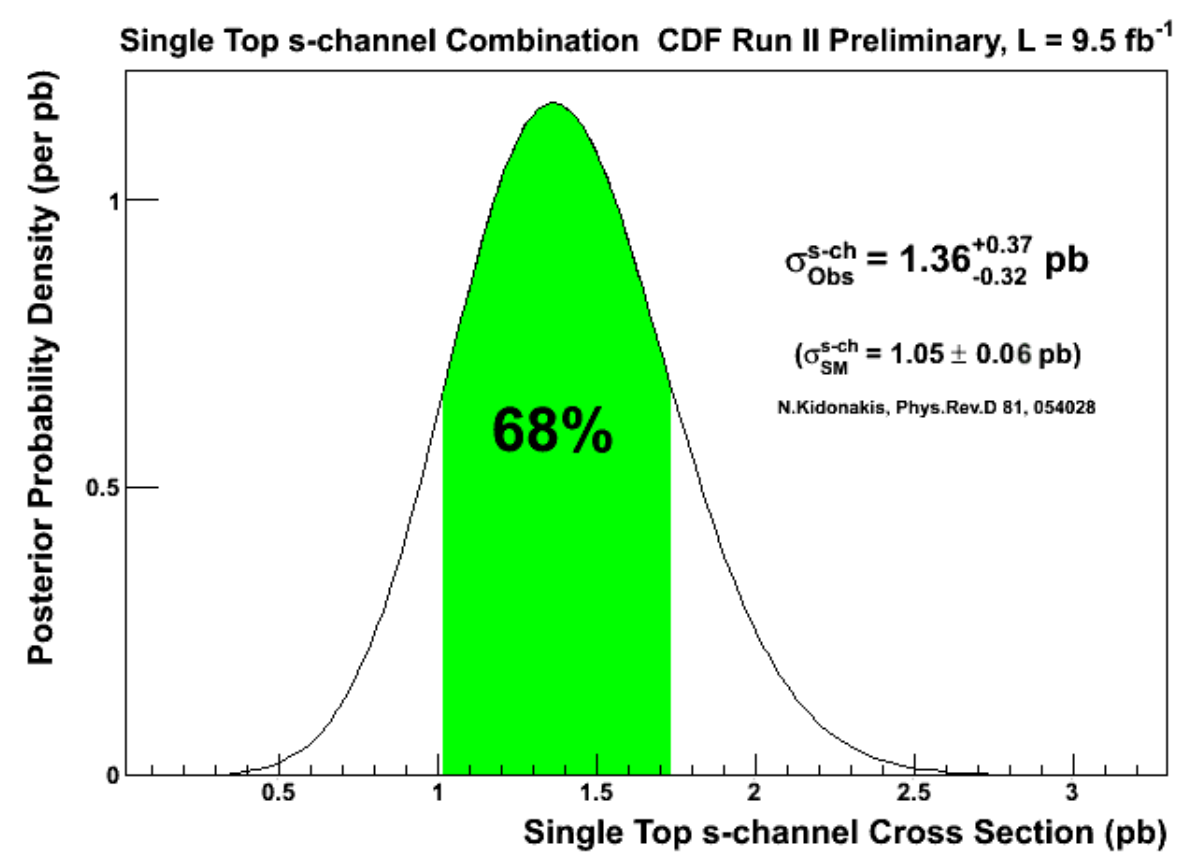

Figure 6.8 - The posterior probability distribution for the CDF combination.

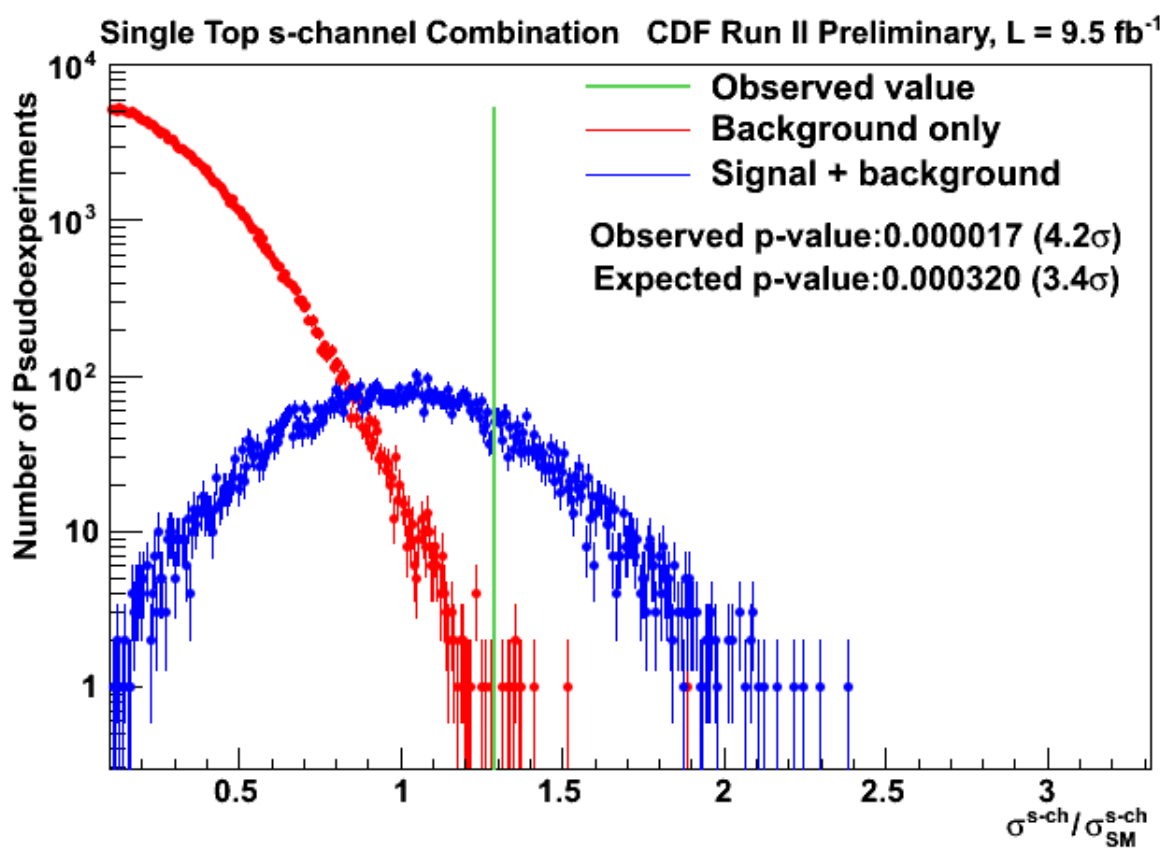

Figure 6.9 - Pseudo-experiment distributions for the background-only (red) and SM-signalplus-background (blue) hypotheses from the CDF combination.

to model, and to minimize the QCD multijet background. Events passing the selection were further separated into independent analysis channels based on the number of reconstructed jets as well as the number and quality of $b$-tagged jets. 
Multivariate discriminants were formed, optimized for separating the s-channel single top quark signal events in each of the analysis samples from the larger background contributions, to extract the cross section measurements ${ }^{3}$.

The combined Tevatron cross section measurement is performed following the same strategy used for the CDF combination and illustrated in Section 5.1.2, forming a binned likelihood as a product of all analysis channels of both Collaborations. Sources of systematic uncertainty common to measurements of both Collaborations are assumed to be $100 \%$ correlated, while other uncertainties are assumed to be uncorrelated, as summarized in Table 6.3.

Table 6.3 - Systematic uncertainties associated with the CDF and D0 single top quark schannel cross section measurements. The values shown for each category indicate the range of uncertainties applied to the predicted normalizations for signal and background contributions over the full set of analysis samples from each experiment. The black dots indicate which categories contribute uncertainties on the shape of the final multivariate discriminant output variable. It is also noted if categories are treated as fully correlated between the two experiments.

\begin{tabular}{lccccc}
\hline \hline Systematic uncertainty & \multicolumn{2}{c}{ CDF } & \multicolumn{2}{c}{ D0 } & Correlated \\
& Rate & Shape & Rate & Shape & \\
\hline Lumi from detector & $4.5 \%$ & & $4.8 \%$ & & \\
Lumi from cross section & $4.0 \%$ & & $3.8 \%$ & & Yes \\
Signal modeling & $2-10 \%$ & $\bullet$ & $3-8 \%$ & & Yes \\
Background (simulation) & $2-12 \%$ & $\bullet$ & $2-11 \%$ & $\bullet$ & Yes \\
Background (data) & $15-40 \%$ & $\bullet$ & $19-50 \%$ & $\bullet$ & \\
Detector modeling & $2-10 \%$ & $\bullet$ & $1-5 \%$ & $\bullet$ & \\
$b$-tagging & $10-30 \%$ & & $15-40 \%$ & $\bullet$ & \\
JES & $0-20 \%$ & $\bullet$ & $9-40 \%$ & $\bullet$ & \\
\hline \hline
\end{tabular}

Figure 6.10 shows the signal and background expectations and the data as a function of $\log _{10}(s / b)$ of the collected bins, for the combined CDF and D0 analyses. The respective background-subtracted $\log _{10}(s / b)$ discriminant distribution using the most likely values for the signal and background yields derived from the likelihood fit is shown in Figure 6.11.

The extracted posterior probability distribution for $\sigma_{\mathcal{S}}$ is presented in Figure 6.12, and Figure 6.13 gives a graphical presentation of the individual and combined measurements. All measurements agree within their uncertainties with the SM prediction of $1.05 \pm$ $0.06 \mathrm{pb}$ [22]. The most probable value for the combined cross section is $1.29_{-0.24}^{+0.26} \mathrm{pb}$ for a top-quark mass of $172.5 \mathrm{GeV}$.

\footnotetext{
${ }^{3}$ For the D0 Collaboration this is different from Ref. [98], where a combined $s$ - and $t$-channel discriminant was used to measure both single top quark channels separately, without assuming their SM cross sections. For this combination, a discriminant where the $s$-channel is the only signal and the $t$-channel is considered as background normalized to its SM cross section is used.
} 


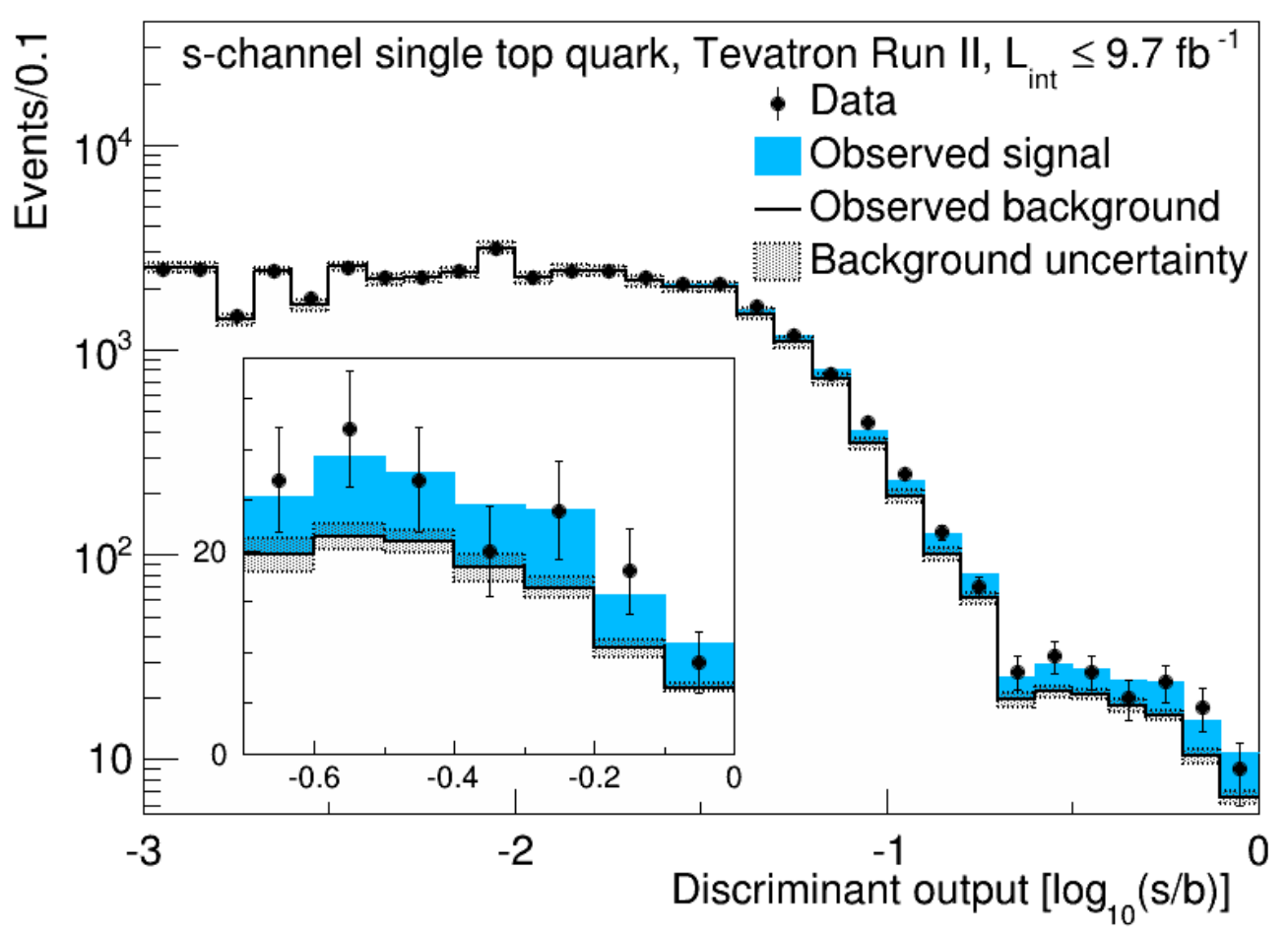

Figure 6.10 - Distribution of the discriminant histograms, summed for bins with similar signal-to-background ratio $(s / b)$. The expected sum of the backgrounds is shown by the unfilled histogram, and the total uncertainty of the background is represented by the gray shaded band. The expected s-channel signal contribution is shown by a filled blue histogram.

The total expected uncertainty is $20 \%$, and the expected uncertainty without considering systematic uncertainties is $14 \%$. The dependence of the measured value on the assumed value of the top quark mass is estimated to be negligible compared to the uncertainty on the measurement [78].

The statistical significance of this result is quantified through a calculated $p$-value based on an asymptotic approximation, using the log-likelihood ratio as test statistic (see Section 5.3). The distributions of the log-likelihood ratio resulting from fits to pseudo-data that include background-only, or signal-plus-background, contributions are presented in Figure 6.14. A $p$-value of $1.8 \times 10^{-10}$ is calculated, corresponding to a significance of 6.3 standard deviations, to be compared to the expected sensitivity of 5.1 standard deviations. The existence of an s-channel contribution to the single top quark production is herewith established. 


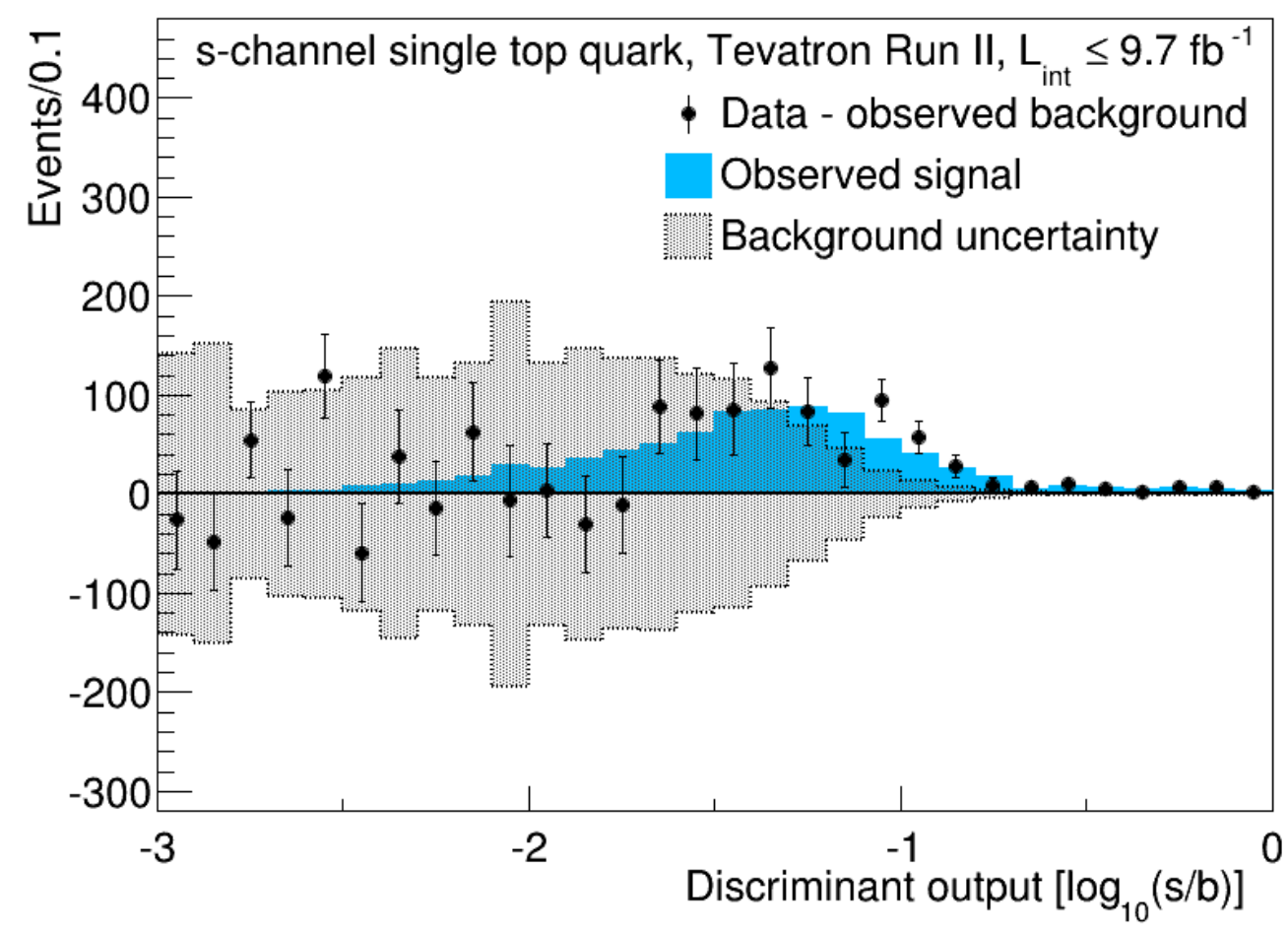

Figure 6.11 - The background-subtracted distribution of the discriminant histograms, summed for bins with similar signal-to-background ratio $(s / b)$. The background and $s-$ channel signal (blue filled histogram) have been normalized to the most likely values returned from the likelihood fit and the uncertainty on the background uncertainty has also been constrained by the data (gray shaded band).

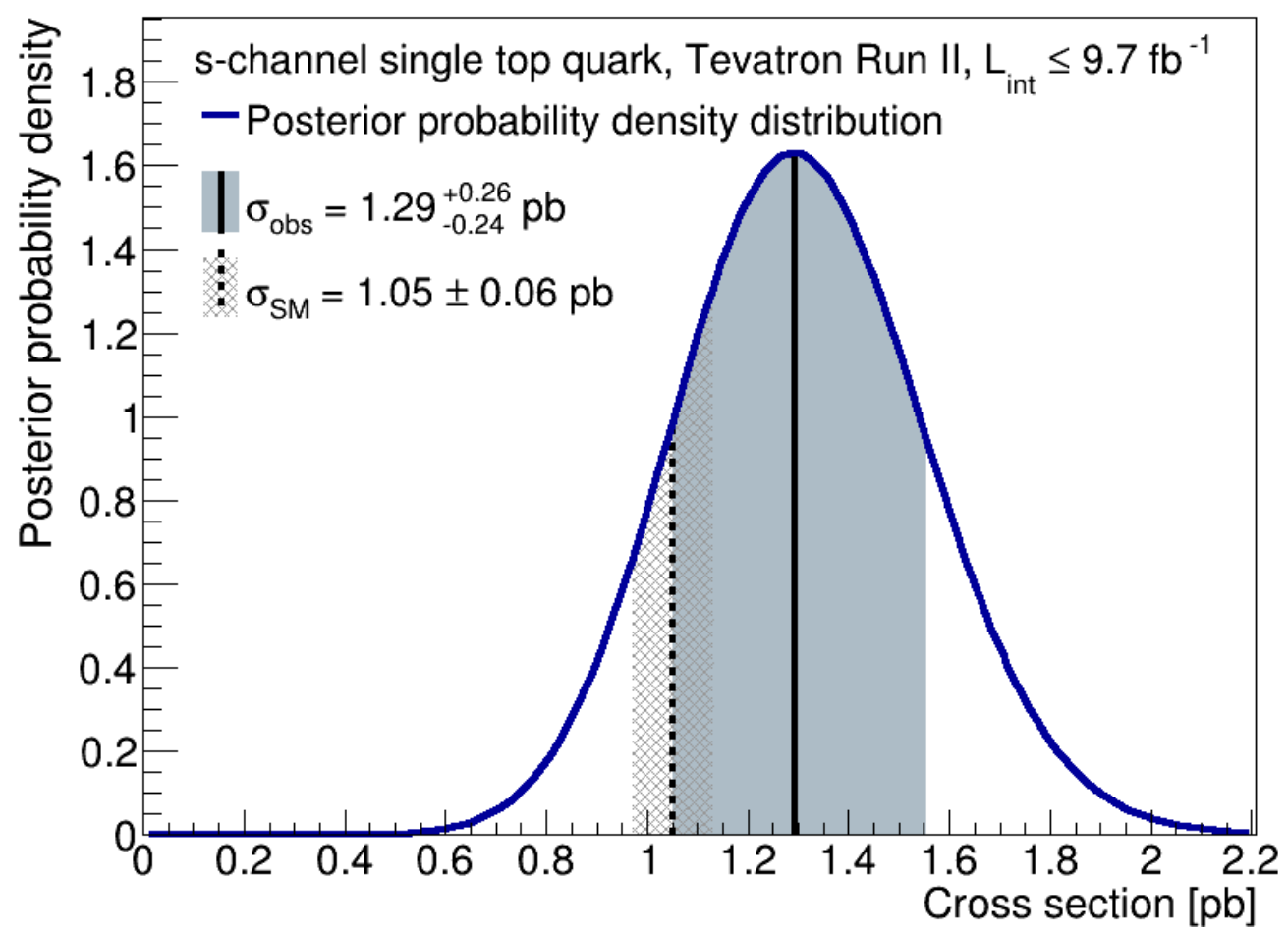

Figure 6.12 - The posterior probability distribution for the combination of the CDF and D0 analysis channels compared with the theoretical prediction [22]. 


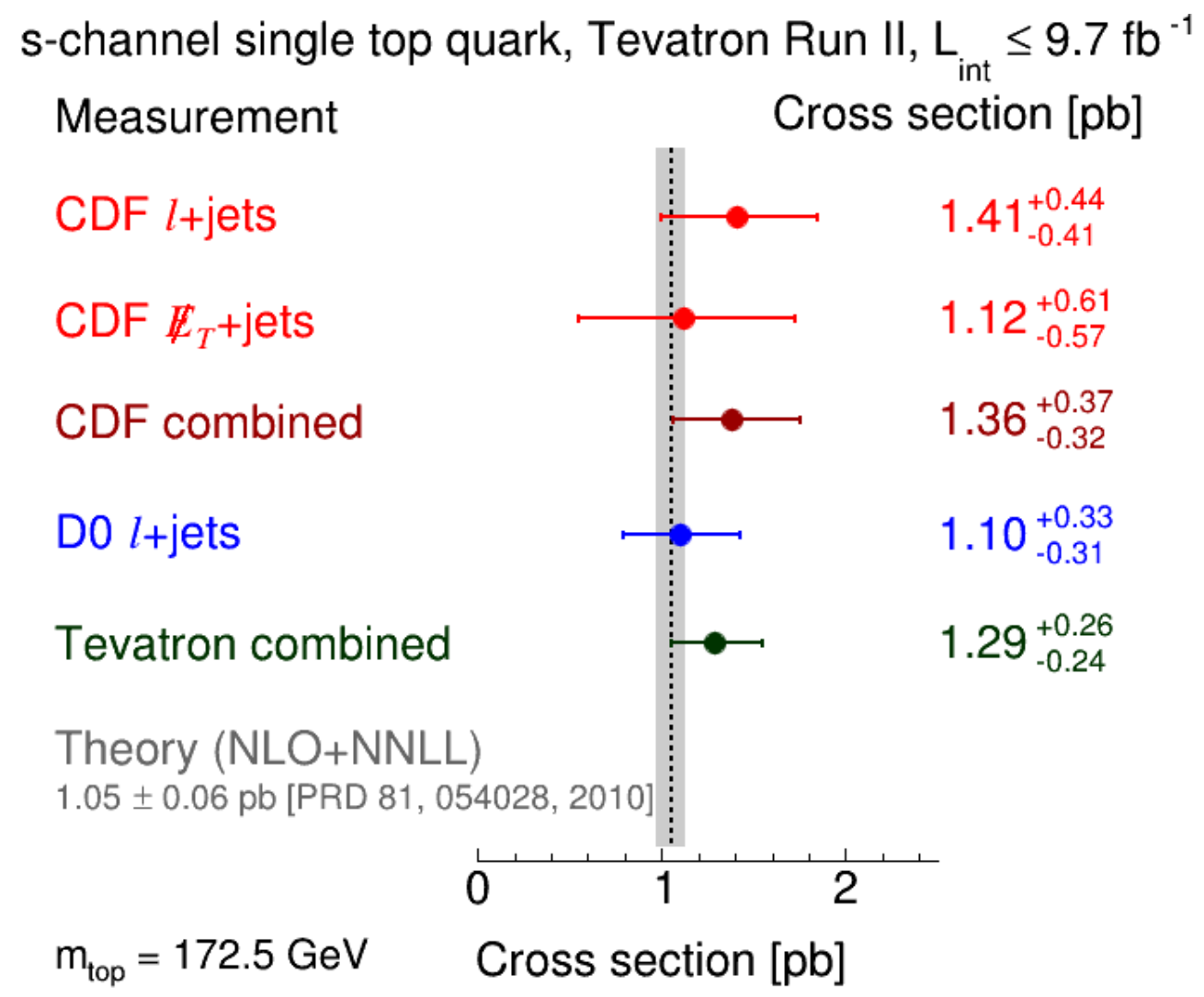

Figure 6.13 - Measured single-top-quark s-channel production cross sections from each of the individual analyses and various combinations of these analyses compared with the theoretical prediction [22]. 


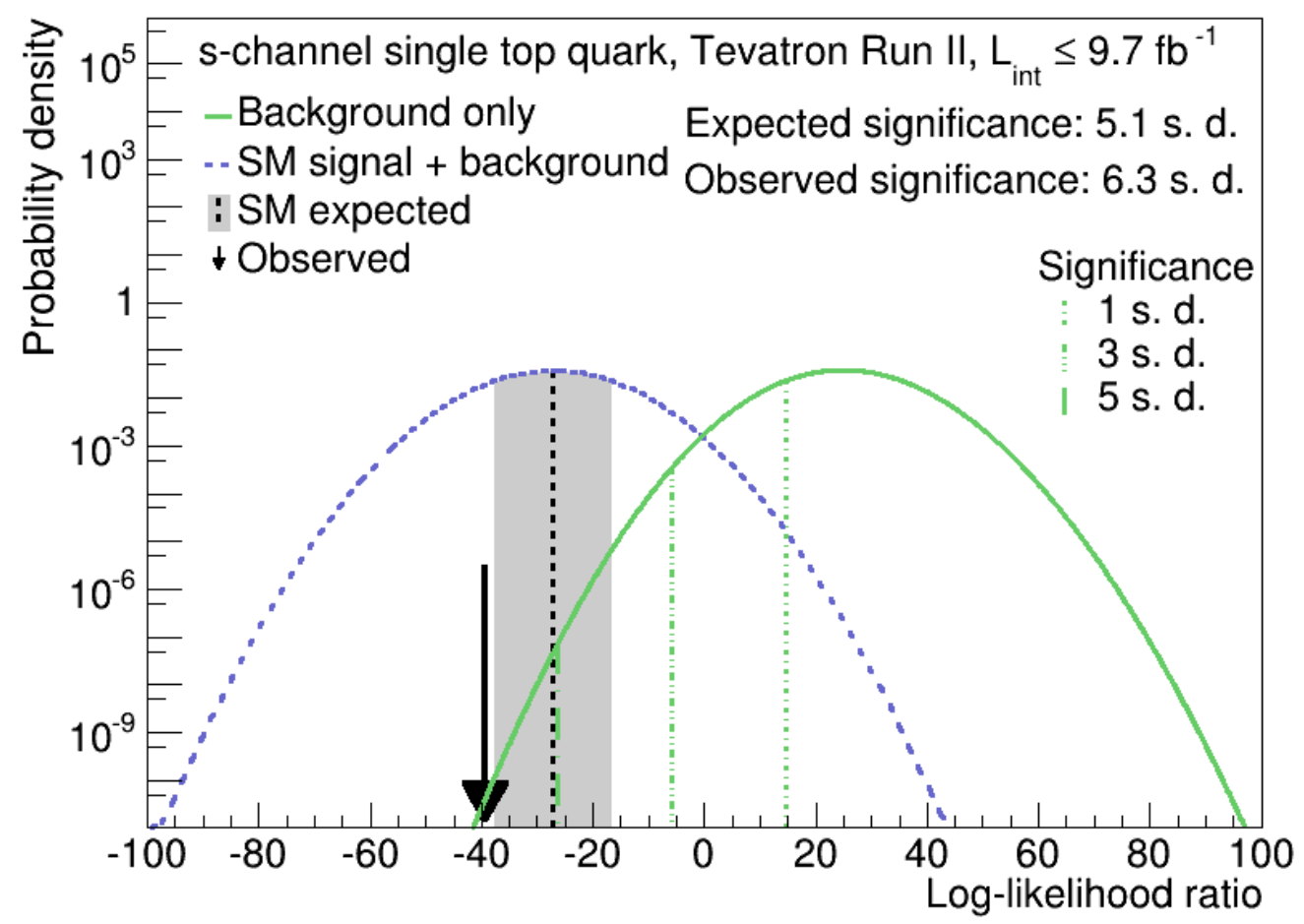

Figure 6.14 - Log-likelihood ratios using an asymptotic approximation for the backgroundonly (solid green line) and SM-signal-plus-background (dashed blue) hypotheses from the combined measurement. 


\section{Chapter 7}

\section{Search for New Resonances Decaying to a Top and a Bottom Quark}

Short-lived massive states decaying to pairs of SM leptons or quarks are included in several modifications of the SM, such as $S U(2)_{R}$ SM extensions [41], Kaluza-Klein extradimensions [38], technicolor [39] or Little Higgs scenarios [40] where a resonance decaying to $a t b$ pair (where $t b$ indicates both the state $\bar{t} \bar{b}$ and its charge conjugate $\bar{t} b$ ) can appear. Searches for $W^{\prime}$ bosons in the $W^{\prime} \rightarrow t b$ decay channel are complementary to searches in the leptonic decay channel $W^{\prime} \rightarrow \ell v$, and can probe cases where the couplings of the $W^{\prime}$ to fermions favour quarks versus leptons.

In the recent past, searches in the $W^{\prime} \rightarrow t b$ channel have been performed by the CDF [37] and D0 [104] experiments at the Tevatron, and by the ATLAS [105] and CMS [106] experiments at the LHC. The LHC experiments have superior sensitivity to the Tevatron for searches at the highest masses due to the higher center-of-mass energy. However, the Tevatron experiments have competitive sensitivity in the lower mass region $\left(M_{W^{\prime}}<\right.$ $700 \mathrm{GeV} / c^{2}$ ) due to the more favourable signal-to-background ratio in searches for particles produced in quark-initiated states, such as the $W^{\prime}$ boson, with respect to the SM background processes which are mainly gluon initiated.

In this Chapter we present a new search for $W^{\prime}$-like resonances decaying to $t b$ in events where $t \rightarrow W b$ and the $W$ decays to a charged lepton-neutrino pair.

A simple left-right symmetric SM extension [107] is used as a benchmark model, predicting the existence of $W^{\prime}$ bosons of unknown mass and universal weak coupling strength. This search is sensitive to any narrow resonant state decaying to $t b$ since no specific assumptions on the signal model are made. 


\subsection{Definition of the Analysis Subsamples}

The event selection applied in this search is similar to the one applied in the s-channel single top quark search reported in Chapter 6. Since we are looking for massive resonances decaying in a $t$ and in a $b$-quark, the minimum values required for the $\mathbb{E}_{T}$ and jet energies are higher with respect to the selection criteria used in the s-channel analysis. Offline, $E_{T}>50 \mathrm{GeV}$ is required. Events with two or three high- $E_{T}$ jets are selected and the two jets with the largest transverse energies, $E_{T}^{j_{1}}$ and $E_{T}^{j_{2}}$, are required to satisfy $E_{T}^{j_{1}}>35 \mathrm{GeV}$ and $E_{T}^{j_{2}}>25 \mathrm{GeV}$. Events satisfying the EWK selection criteria described in Section 4.2.2 are also considered as signal-sensitive events. In this search, events containing identified electrons or muons are thus analyzed. At this stage of the analysis, 941 predicted signal events, simulated considering a $W^{\prime}$ boson mass of $300 \mathrm{GeV}$, are accepted by the selection, compared to a total number of data events of 391,229 . In order to identify $b$ jets, the SECVTX 3.2.1 and JETPROB 3.2.2 algorithms are used. The choice of SECVTX and JETPROB instead of the HOBIT tagger used in Chapter 6 is due to the fact that a large contribution from events with high energy jets is expected in this analysis, while HOBIT is not able to distinguish light-flavour jets from heavy-flavour jets if the jet energy is larger than about $200 \mathrm{GeV}$, as explained in Section 6.1.1.

At least one of the first two leading jets in $E_{T}$ is required to be tagged by SECVTX. Events are further divided among twelve statistically independent regions, depending on whether the other leading jet is not tagged (1T), tagged by JETPROB but not by SECVTX (TL), and tagged by SECVTX (TT); the number of jets (two-jet or three-jet sample) and the presence of at least one reconstructed electron or muon (no-lepton and lepton sample) The signalto-background ratio and background composition are different among the subsamples, resulting in an increased sensitivity. At this stage of the analysis 483 predicted signal events for a $W^{\prime}$ boson mass of $300 \mathrm{GeV}$ are accepted by the selection, compared to a total number of data events of 25,256 .

\subsection{Multivariate Discriminants}

An artificial neural network, $\mathrm{NN}_{\mathrm{QCD}}$, is employed to reject the dominant $\mathrm{QCD}$ multijet background, separating it from signal and other backgrounds. The same $\mathrm{NN}_{\mathrm{QCD}}$ function trained to separate $W+$ jets events from the QCD multijet background in the s-channel single top quark search (see Section 6.1.2) is used. This is justified since the final state topologies between $W^{\prime}$ boson and s-channel single top quark production are very similar. As no information on the $W^{\prime}$ mass is included in the training sample, this also ensures a smooth performance in QCD multijet background separation across the whole $W^{\prime}$ boson mass range.

Events are required to satisfy a minimum $\mathrm{NN}_{\mathrm{QCD}}$ requirement. To determine the ap- 
propriate normalization in each different analysis subsample, a scale factor is derived in the region composed by the rejected events, where the tagged diboson, top and $W / Z+$ jets background estimates are subtracted from the tagged data. Table 7.1 and Table 7.2 show expected event yields for background processes, observed data events, and expected number of events for one signal hypothesis.

Table 7.1 - Numbers of predicted and observed two-jet events with and without identified leptons, in the 1T, TL, and TT subsamples. The uncertainties on the predicted numbers of events are due to the theoretical and experimental uncertainties on signal and background modeling. Expected number of events for a right-handed $W^{\prime}$ boson with SM-like couplings and $300 \mathrm{GeV} / c^{2}$ mass are shown.

\begin{tabular}{lccc}
\hline \hline Category & $1 \mathrm{~T}$ & TL & TT \\
\hline$W^{\prime}\left(300 \mathrm{GeV} / c^{2}\right)$ & $156 \pm 10$ & $59.9 \pm 4.6$ & $84.6 \pm 7.9$ \\
$s$-ch. single top & $98.3 \pm 9.8$ & $36.4 \pm 3.8$ & $46.1 \pm 4.3$ \\
$t$-ch. single top & $167 \pm 24$ & $7.3 \pm 1.1$ & $7.9 \pm 1.1$ \\
$t \bar{t}$ & $457 \pm 32$ & $141 \pm 11$ & $177 \pm 12$ \\
$V V$ & $259 \pm 18$ & $28.5 \pm 2.0$ & $27.0 \pm 2.0$ \\
$V H$ & $14.0 \pm 1.2$ & $5.4 \pm 0.5$ & $7.2 \pm 0.5$ \\
$V+$ jets & $3472 \pm 901$ & $236 \pm 61$ & $157 \pm 39$ \\
QCD & $2766 \pm 103$ & $220 \pm 17$ & $102 \pm 12$ \\
\hline Total background & $7235 \pm 908$ & $674 \pm 64$ & $524 \pm 43$ \\
Observed & 7128 & 680 & 507 \\
\hline \hline
\end{tabular}

Table 7.2 - Numbers of predicted and observed three-jet events with and without identified leptons, in the 1T, TL, and TT subsamples. The uncertainties on the predicted numbers of events are due to the theoretical and experimental uncertainties on signal and background modeling. Expected number of events for a right-handed $W^{\prime}$ boson with SM-like couplings and $300 \mathrm{GeV} / c^{2}$ mass are shown.

\begin{tabular}{lccc}
\hline \hline Category & $1 \mathrm{~T}$ & TL & TT \\
\hline$W^{\prime}\left(300 \mathrm{GeV} / c^{2}\right)$ & $79.5 \pm 5.34$ & $23.5 \pm 1.9$ & $28.8 \pm 3.0$ \\
$s$-ch. single top & $50.0 \pm 5.3$ & $13.0 \pm 1.5$ & $16.0 \pm 1.6$ \\
$t$-ch. single top & $90.8 \pm 13.7$ & $5.8 \pm 0.9$ & $6.9 \pm 1.0$ \\
$t \bar{t}$ & $899 \pm 65$ & $148 \pm 12$ & $162 \pm 11$ \\
$V V$ & $106 \pm 8$ & $9.7 \pm 0.7$ & $7.8 \pm 0.6$ \\
$V H$ & $6.3 \pm 0.5$ & $1.7 \pm 0.2$ & $2.1 \pm 0.2$ \\
$V+$ jets & $1360 \pm 357$ & $80.6 \pm 21.2$ & $51.6 \pm 13.4$ \\
QCD multijet & $1261 \pm 64$ & $92.8 \pm 9.4$ & $31.8 \pm 4.6$ \\
\hline Total & $3774 \pm 369$ & $352 \pm 26$ & $278 \pm 18$ \\
Observed & 3613 & 388 & 274 \\
\hline \hline
\end{tabular}

A final discriminant to distinguish each $W^{\prime}$ boson mass hypothesis from the remaining backgrounds is derived applying a staged neural network technique. In analogy 
to the strategy described in Section 6.1.2, two additional networks, $\mathrm{NN}_{V \text { jets }}$ and $\mathrm{NN}_{t \bar{t}}$, are trained for events that satisfy the minimum requirement on the $\mathrm{NN}_{\mathrm{QCD}}$ output variable. The first, $\mathrm{NN}_{V \text { jets, }}$ is trained to separate the $W^{\prime}$ boson signal from $V+$ jets and the remaining QCD backgrounds. In the training, a simulated $W^{\prime}$ signal is used, while the background sample consists of pretag data events that satisfy the requirement on $\mathrm{NN}_{\mathrm{QCD}}$, reweighted by the tag-rate probability as derived from the TRM (see Section 4.3.3). The second, $\mathrm{NN}_{t \bar{t}}$, is trained to separate $W^{\prime}$ boson from $t \bar{t}$ production using simulation for both components. Variables which describe the energy and momentum flow in the detector and angular variables are used in the training of the $\mathrm{NN}_{V \text { jets }}$ and $\mathrm{NN}_{t \bar{t}}$ discriminants. Some of the most separating variables used in the training of the $\mathrm{NN}_{V \text { jets }}$ and $\mathrm{NN}_{t \bar{t}}$ discriminants, as well as the ones used in the training of the $\mathrm{NN}_{\mathrm{QCD}}$, are shown in Figures 7.1 - 7.4:
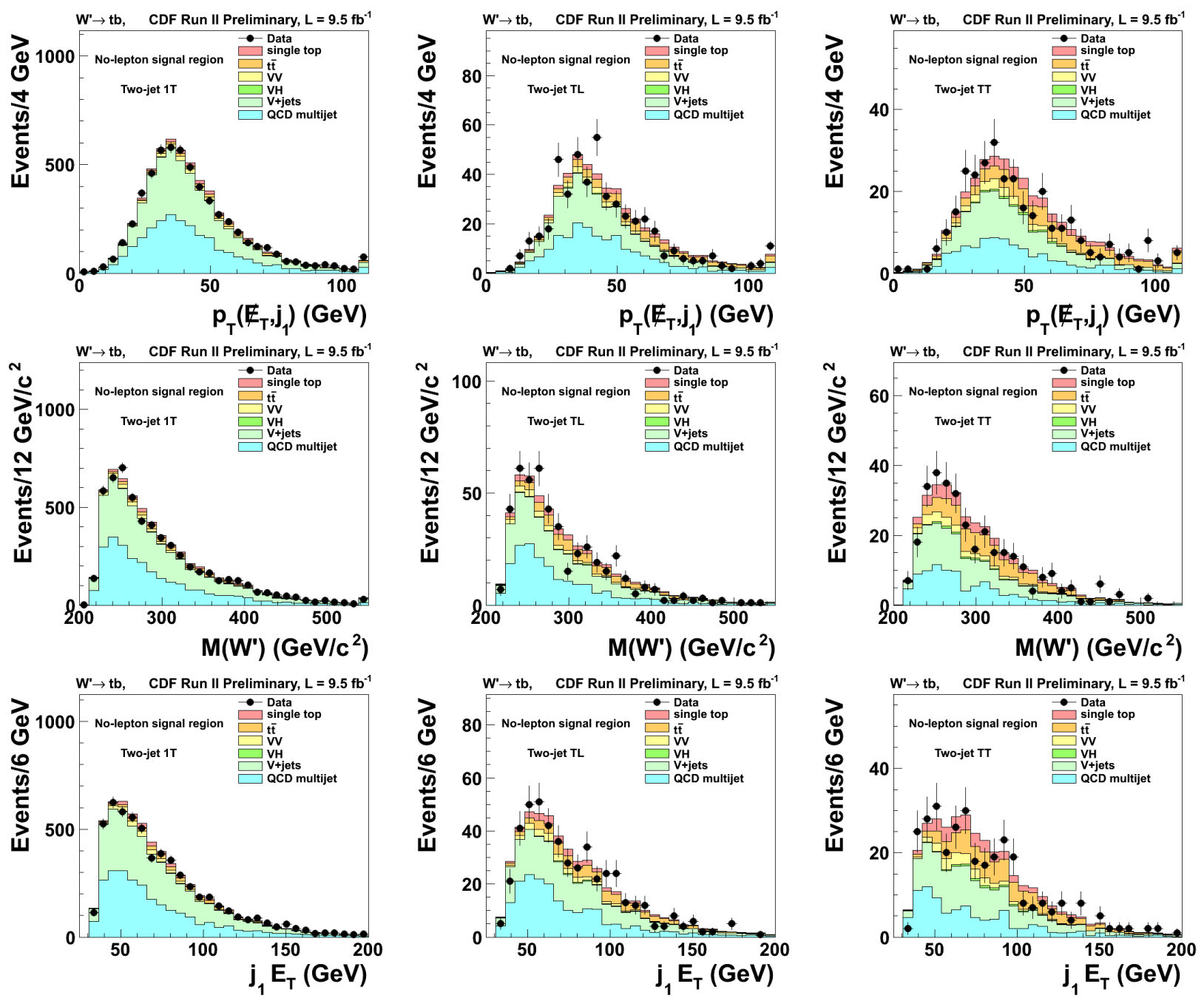

Figure 7.1 - Predicted and observed distributions in the signal region for some of the most separating kinematic variables used in the training of the $\mathrm{NN}_{\mathrm{QCD}}, \mathrm{NN}_{V j \text { jets }}$ and $\mathrm{NN}_{t \bar{t}}$ discriminants in the no-lepton two-jet subsample.

The final discriminant, $\mathrm{NN}_{\text {sig }}$, is defined as the quadrature sum of the $\mathrm{NN}_{V \text { jets }}$ and $\mathrm{NN}_{t \bar{t}}$ output variables, both weighted by an appropriate weight optimized to improve the sen- 

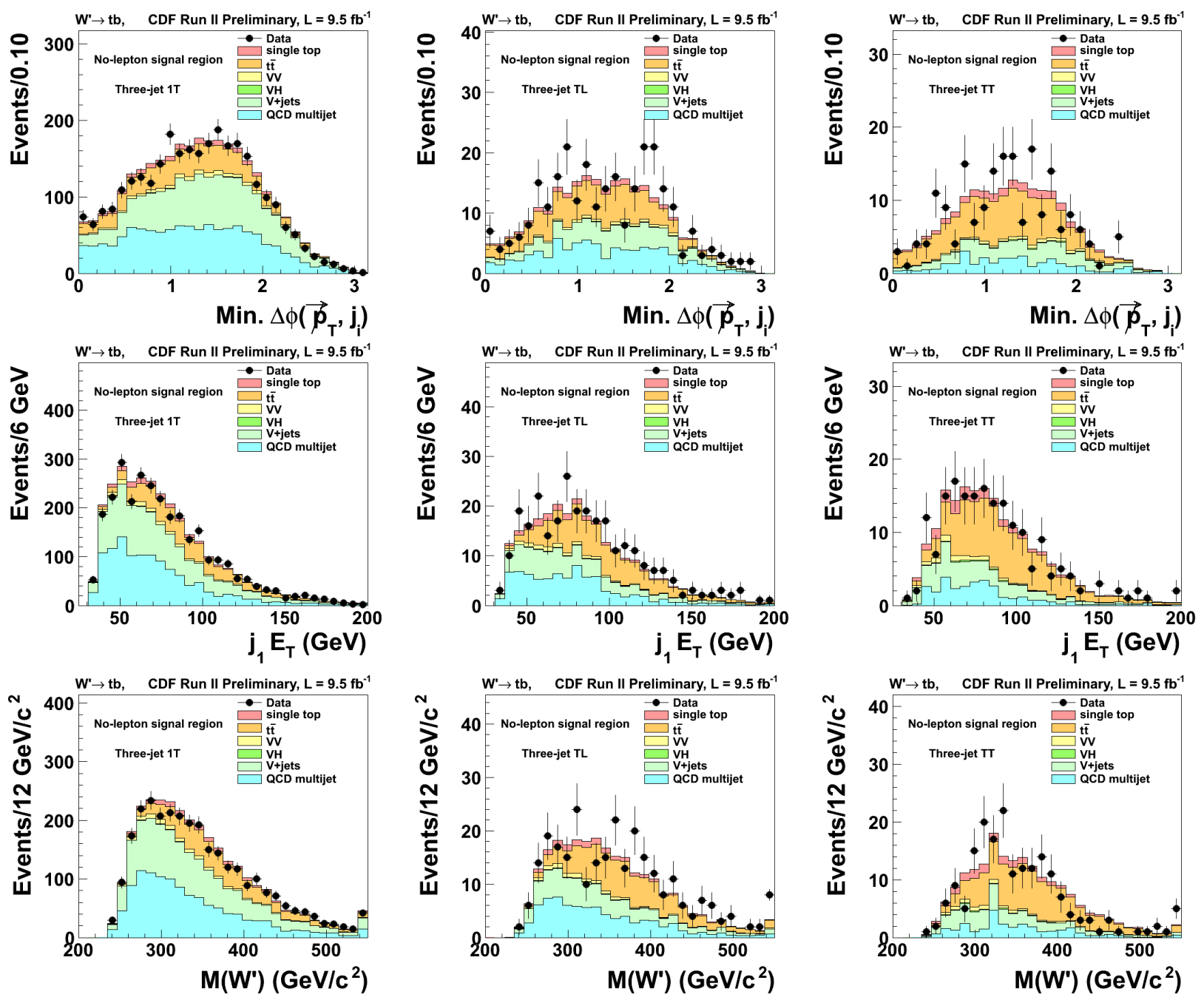

Figure 7.2 - Predicted and observed distributions in the signal region for some of the most separating kinematic variables used in the training of the $\mathrm{NN}_{\mathrm{QCD}}, \mathrm{NN}_{V j \mathrm{jets}}$ and $\mathrm{NN}_{t \bar{t}}$ discriminants in the no-lepton three-jet subsample.

sitivity in each analysis subsample, taking into account the different background contributions. Figure 3.6 shows the predicted and observed shapes of the $300 \mathrm{GeV} W^{\prime} \mathrm{NN}_{\text {sig }}$ output variable.

To validate the background modeling, we compare data and the corresponding combined background prediction in a QCD control sample composed by the events rejected by the minimum requirements on the $\mathrm{NN}_{\mathrm{QCD}}$ output variable. The validation on a variety of kinematic, angular, and event-shape variables in this control samples and in the signal sample are presented in Appendix C. 

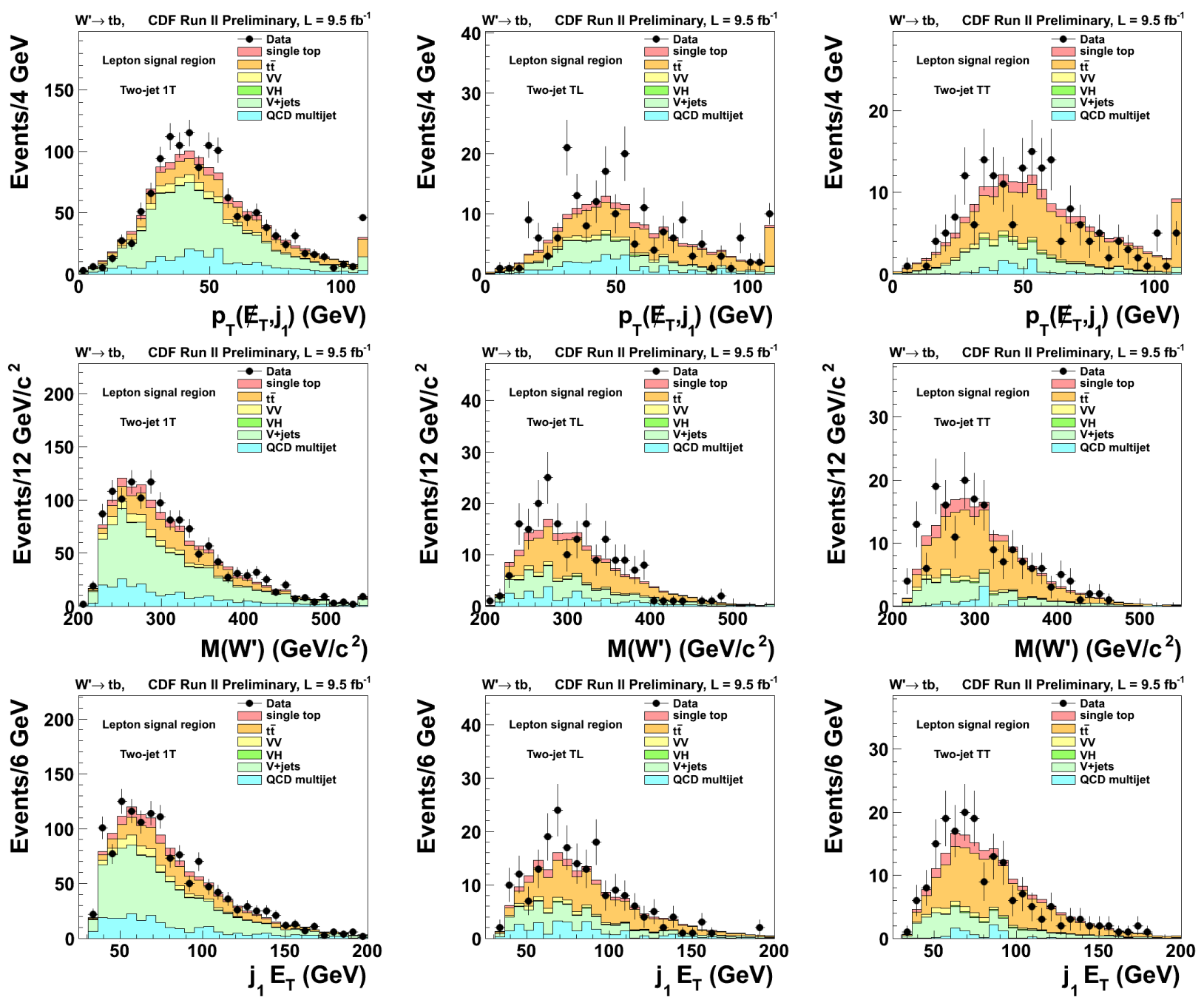

Figure 7.3 - Predicted and observed distributions in the signal region for some of the most separating kinematic variables used in the training of the $\mathrm{NN}_{\mathrm{QCD}}, \mathrm{NN}_{V \text { jets }}$ and $\mathrm{NN}_{t \bar{t}}$ discriminants in the lepton two-jet subsample.

\subsection{Limit Extraction}

A binned likelihood fit is performed to probe for a $W^{\prime} \rightarrow t b$ signal in the presence of SM backgrounds. A Bayesian likelihood method, with a flat, non-negative, prior probability for the $W^{\prime}$ boson production cross section times branching fraction, $\sigma(p \bar{p} \rightarrow$ $\left.W^{\prime}\right) \times \mathcal{B}\left(W^{\prime} \rightarrow t b\right)$, and truncated Gaussian priors for the uncertainties on the acceptance and shape of the backgrounds, is applied for all $W^{\prime}$ boson mass hypotheses, obtaining 95\% C.L. upper limits on $\sigma\left(p \bar{p} \rightarrow W^{\prime}\right) \times \mathcal{B}\left(W^{\prime} \rightarrow t b\right)$ as a function of $M_{W^{\prime}}$, using the methodology described in Section 5.2. The twelve analysis subsamples are combined by taking the product of their likelihoods and simultaneously varying the correlated uncertainties, as described in Section 5.1.2.

The systematic uncertainties considered in the fit are identical to those used in Section 6.1.3, where the uncertainty due to the efficiencies of the lepton antiselection criteria $(2 \%)$ is 

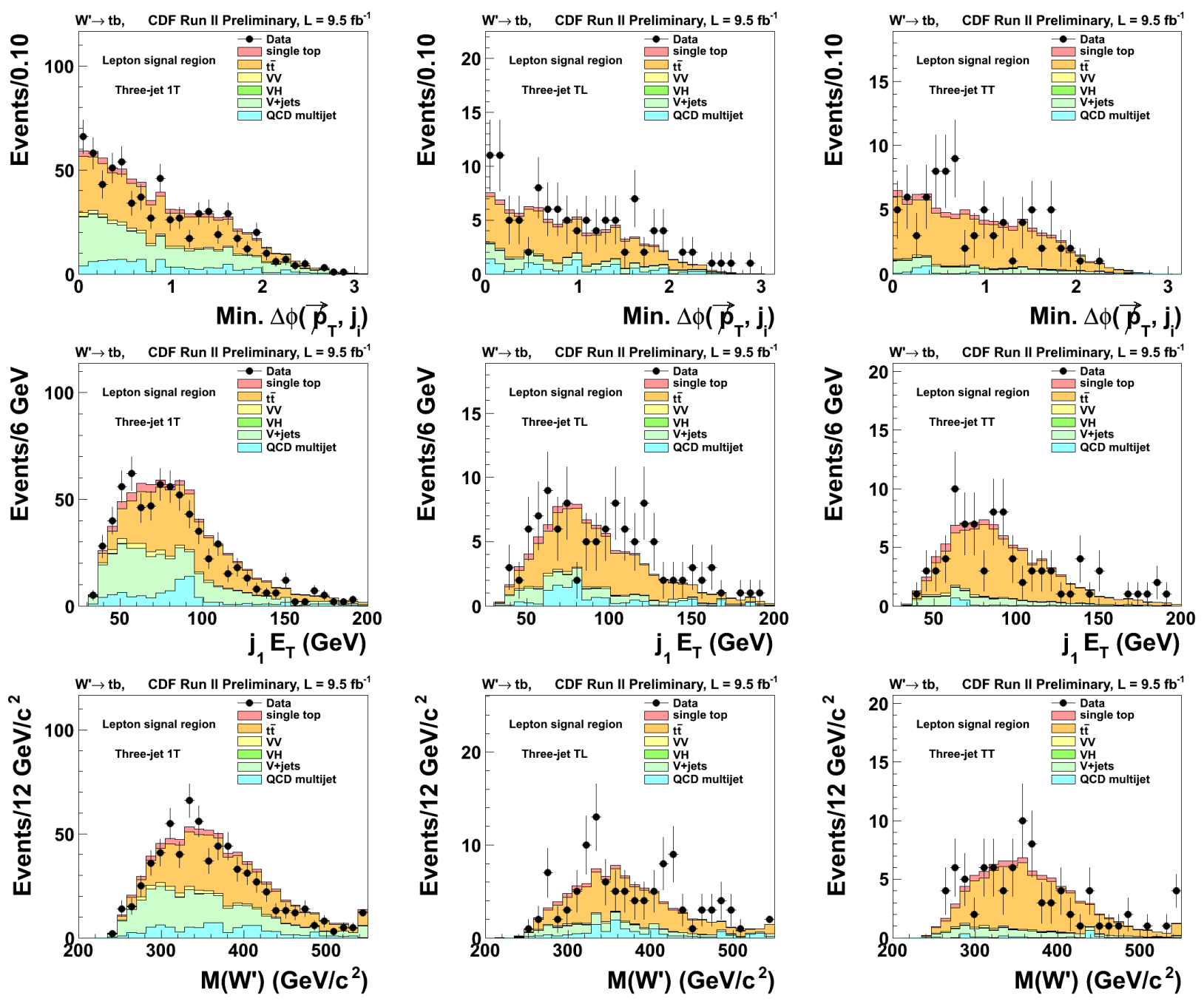

Figure 7.4 - Predicted and observed distributions in the signal region for some of the most separating kinematic variables used in the training of the $\mathrm{NN}_{\mathrm{QCD}}, \mathrm{NN}_{V j \mathrm{jets}}$ and $\mathrm{NN}_{t \bar{t}}$ discriminants in the lepton three-jet subsample.

considered in this analysis as the uncertainty on lepton reconstruction efficiency. The schannel single top quark production is considered as a background for this search and the uncertainty on its normalization (5\%) is also included. Since the SECVTX and the JETPROB taggers are used, the uncertainty on the $b$-tagging efficiency ranges from 8 to $16 \%$. An additional uncertainty on the $b$-tagging efficiency is applied to signal templates as an $E_{T}$-dependent term, to properly take into account the uncertainty in the extrapolation of the $b$-tagging scale factor to the high- $E_{T}$ regions typical of $W^{\prime} \rightarrow t b$ events [37].

The expected and observed upper limits are shown in Figure 7.6. The observed limits are compatible with the expectations calculated assuming that no $W^{\prime} \rightarrow t b$ signal is present in the data. By comparing the limits on $\sigma\left(p \bar{p} \rightarrow W^{\prime}\right) \times \mathcal{B}\left(W^{\prime} \rightarrow t b\right)$ with the theoretical NLO calculations for a right-handed $W^{\prime}$ boson with SM-like couplings [107], $W^{\prime}$ bosons with masses less than $860(880) \mathrm{GeV} / c^{2}$ are excluded, in cases where decay to leptons is allowed (forbidden). 

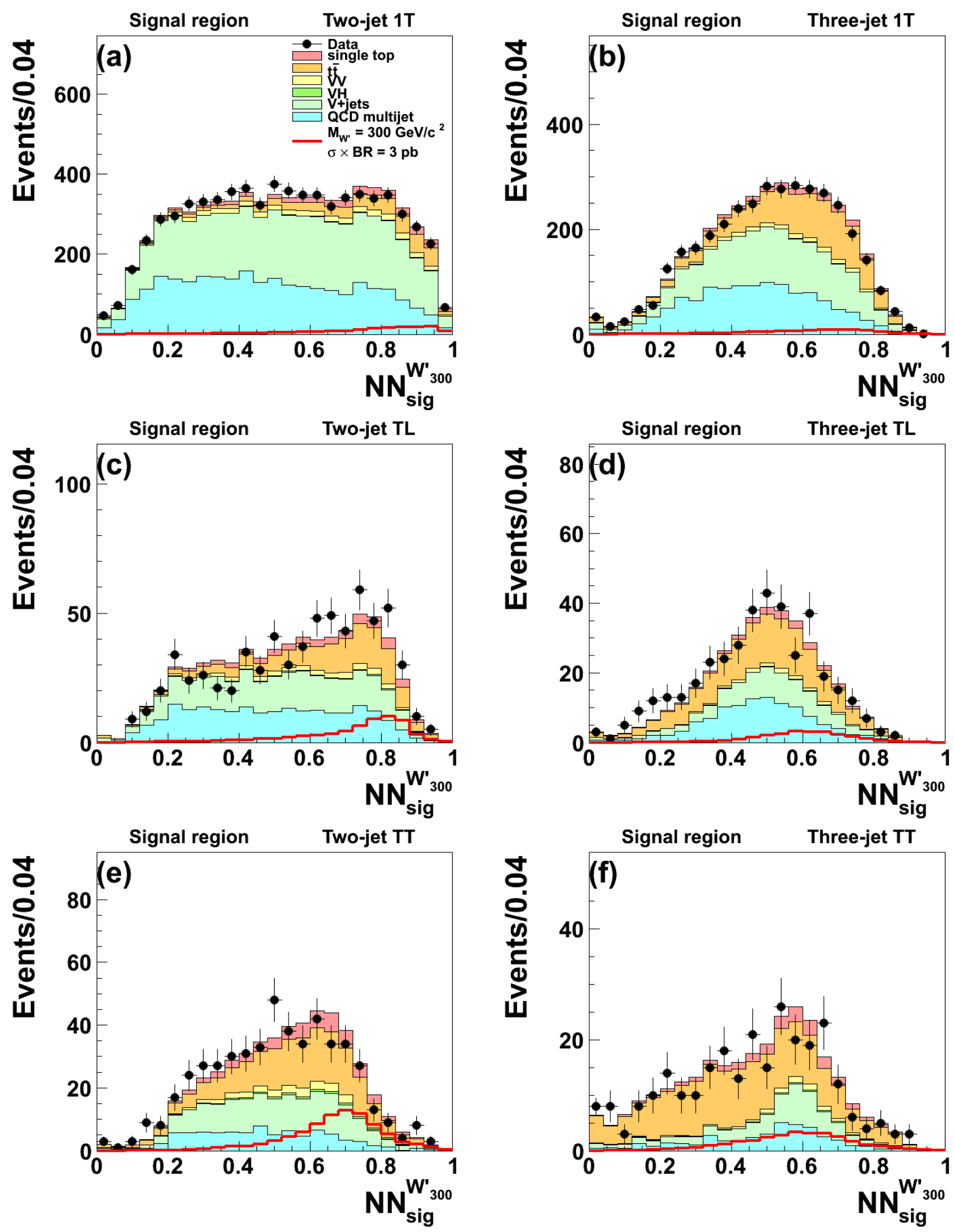

Figure 7.5 - Predicted and observed final discriminant distributions in the signal region, for (a) 1T two-jet, (b) 1T three-jet, (c) TL two-jet, (d) TL three-jet, (e) TT two-jet and (f) TT threejet event subsamples. Events from the no-lepton and lepton subsamples are merged. The distribution for a $W^{\prime}$ boson mass of $300 \mathrm{GeV} / c^{2}$ with $\mathrm{SM}$ coupling is superimposed.

For a simple s-channel production model with effective coupling $g_{W^{\prime}}$, and assuming that couplings to light quarks and heavy quarks are identical, the cross section is proportional 
to $g_{W^{\prime}}^{2}$. Relaxing the assumption of universal weak coupling, the limits on the cross section can be interpreted as upper limits on $g_{W^{\prime}}$ as a function of $M_{W^{\prime}}$. The excluded region of the $g_{W^{\prime}}-M_{W^{\prime}}$ plane is shown in Figure 7.7, with $g_{W^{\prime}}$ expressed in units of $g_{\mathrm{SM}}$. For a value of $M_{W^{\prime}}=300 \mathrm{GeV} / c^{2}$, the effective coupling is constrained at the $95 \%$ C.L. to be less than 0.1 of the $W$ boson coupling.

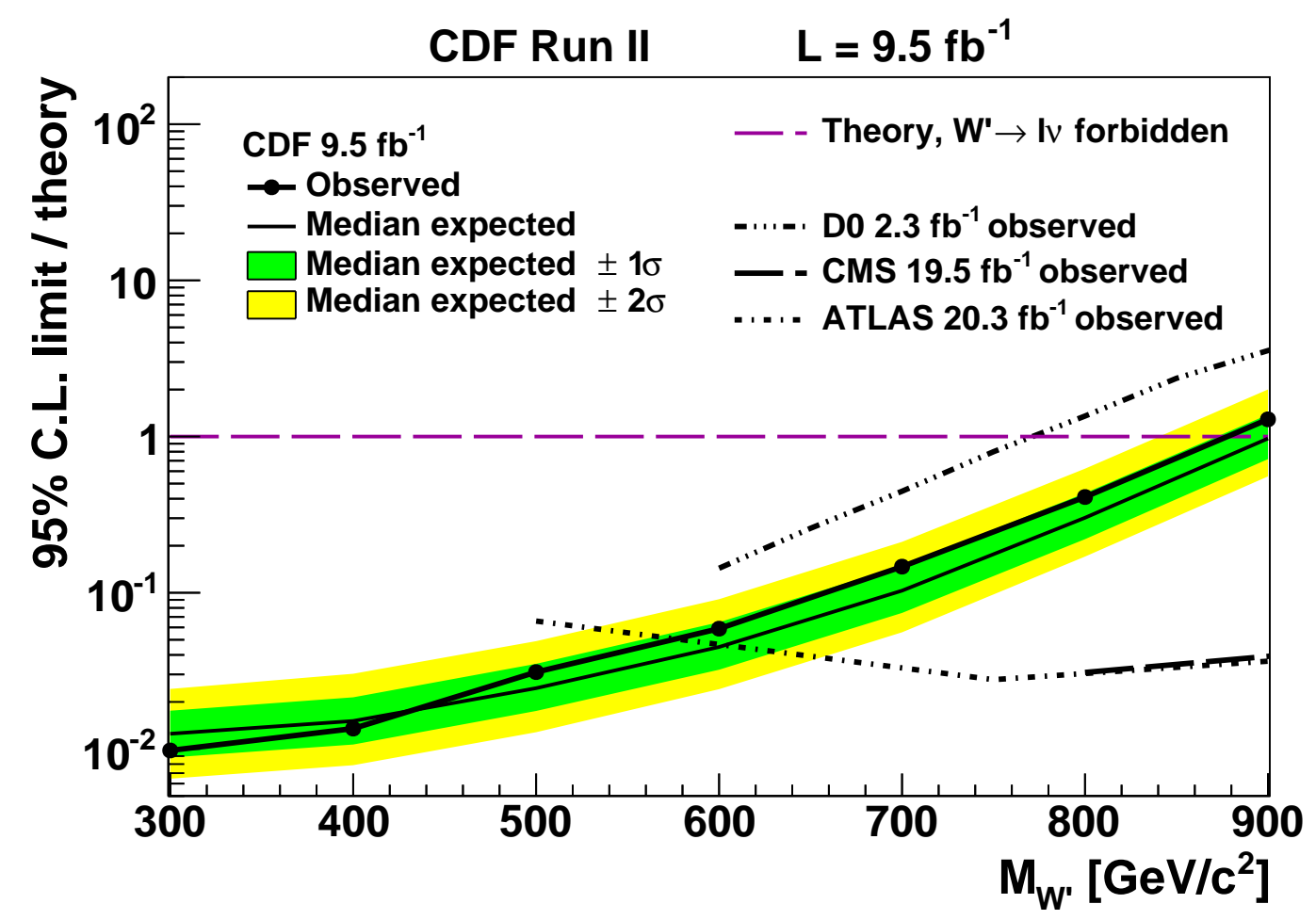

Figure 7.6 - Observed and expected limits on $\sigma\left(p \bar{p} \rightarrow W^{\prime}\right) \times \mathcal{B}\left(W^{\prime} \rightarrow t b\right)$, with $\pm 1 \sigma$ and $\pm 2 \sigma$ confidence intervals and theoretical predictions for a right-handed $W^{\prime}$ with SM-like couplings in cases where the leptonic decay mode $W^{\prime} \rightarrow \ell v$ is allowed (solid line) or forbidden (dashed). The CDF limits are compared with observed limits from the latest $W^{\prime}$ boson searches from ATLAS [105], CMS [106] and D0 [104]. 


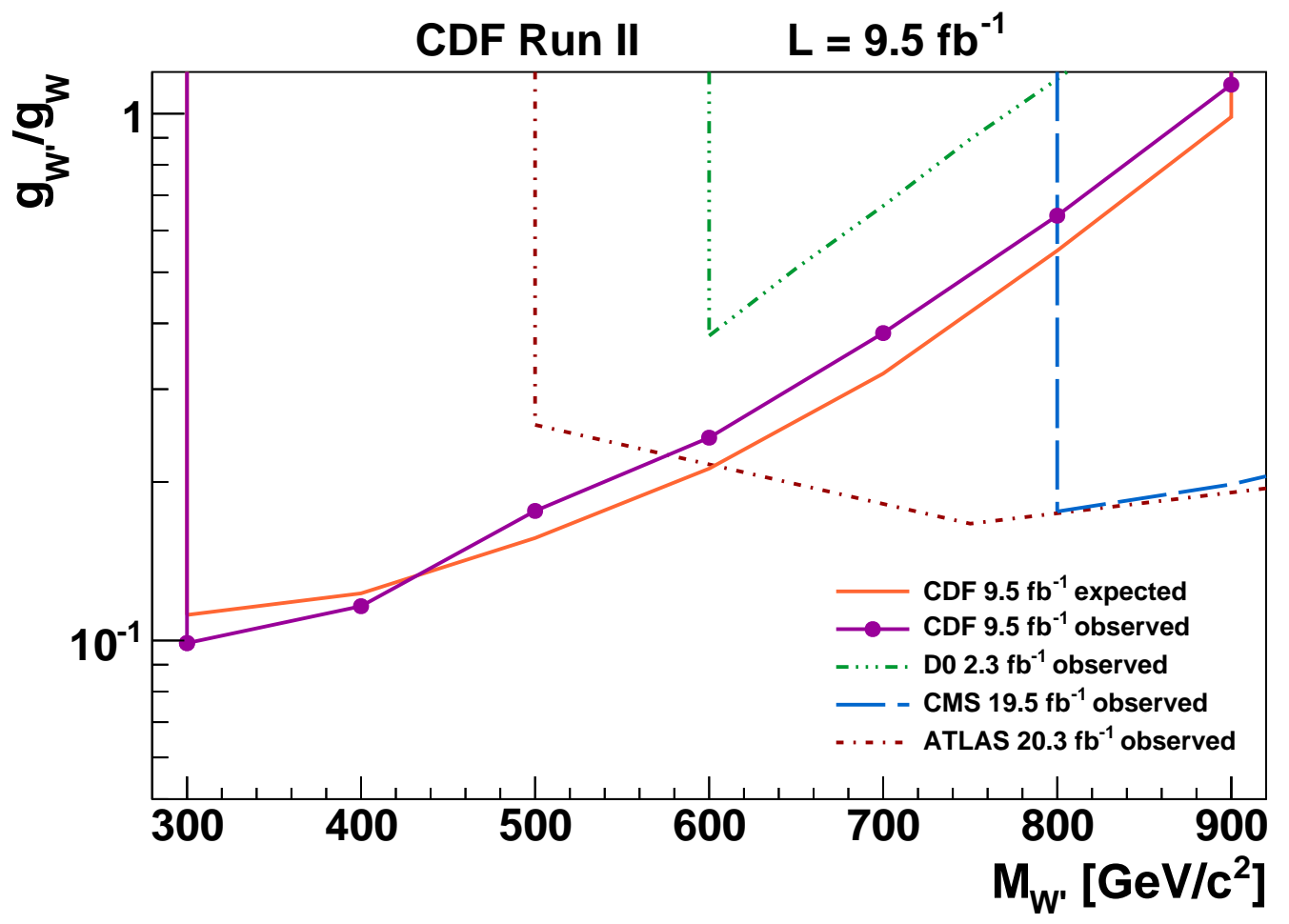

Figure 7.7 - Expected 95\% C.L. limits on the production cross section times branching ratio of a right-handed $W^{\prime}$ with SM coupling strength, normalized to the theoretical cross section times branching ratio as a function of $M_{W^{\prime}}$ in cases where the leptonic decay mode $W^{\prime} \rightarrow \ell v$ is forbidden. The CDF limits are compared with observed limits from the latest $W^{\prime}$ boson searches from ATLAS [105], CMS [106] and D0 [104]. 


\section{Chapter 8}

\section{Conclusions}

In this thesis, the CDF search for s-channel single top quark production, performed for the first time in the $\mathbb{E}_{T} b \bar{b}$ channel, is presented. The result is combined with that from a search in the $\ell v b \bar{b}$ channel [99] to strengthen the reported evidence for s-channel single top quark production, leading to an improvement of more than $10 \%$ on the uncertainty of the measured cross section. We also report the first observation of s-channel single-topquark production with a significance of 6.3 standard deviations by combining the CDF and D0 measurements. The combined value of the s-channel single top quark production cross section is $\sigma_{s}=1.29_{-0.24}^{+0.26} \mathrm{pb}$, in agreement with the SM expectation.

At CDF, a search for a massive resonance decaying to $t b$ is performed with the full CDF II dataset. The data are found to be consistent with the background-only hypothesis, and upper limits are set on the production cross-section times branching ratio at the $95 \%$ confidence level. For a specific benchmark model (left-right symmetric SM extension), in cases where the leptonic decay mode is allowed (forbidden), we exclude $W^{\prime}$ bosons with masses lower than $860(880) \mathrm{GeV} / c^{2}$. For masses smaller than approximately $570 \mathrm{GeV} / c^{2}$, this search yields the most constraining limits to date on the production of narrow $t b$ resonances. 


\section{Appendix A}

\section{Measurement of the Single Top Quark Production Cross Section and the CKM Matrix Element $\left|V_{t b}\right|$ at the Tevatron}

The observation of single top quark production at the Tevatron $[68,93,94]$ was a significant achievement, allowing measurements of the cross section at a hadron collider and improving bounds on the CKM [9] matrix element magnitude $\left|V_{t b}\right|$ due to the direct coupling of the $b$-quark with the singly produced top quark.

Excluding the contribution from the $t W$ production mode, which is expected to be negligible in the final state considered in this Chapter [29], the SM prediction for the combined $s$ - and $t$-channel single top quark production cross section $\sigma_{\mathrm{SM}}^{s+t}$ is $3.15 \pm 0.36 \mathrm{pb}$, which has been calculated including next-to-next-to-leading order corrections [22]. The primary sensitivity to measuring this quantity is usually obtained from events where the $W$ boson from the $t \rightarrow W b$ process decays leptonically to a charged lepton $\ell$ (where $\ell$ represents either an electron $e$ or muon $\mu$ ) and an antineutrino, with a pair of jets, one of which is $b$-tagged (hereafter the " $\ell v b \bar{b}$ " sample) This final state provides a distinctive signature against backgrounds from QCD multijet production, which contain no leptons and multiple jets.

A complementary approach consists in using final states that contain two or three jets and significant $\mathbb{E}_{T}$, which results from the leptonic decay of the $W$ boson, where the lepton is not identified due to reconstruction or acceptance effects and the neutrino carries significant unmeasured momentum. Although QCD multijet events comprise the dominant background in this final state (hereafter the " $E_{T} b \bar{b}$ " analysis or sample), the requirement of significant $E_{T}$ greatly suppresses such background. In addition, this search has sensitivity to events where the $W$ boson decays via $W^{-} \rightarrow \tau^{-} \bar{\nu}_{\tau}$, and the $\tau^{-}$decays hadronically, resulting in a reconstructed jet signature. 


\section{A.1 Measurement of Single Top Quark Production in the $\mathbb{E}_{T} \boldsymbol{b} \bar{b}$ Sample}

The first CDF measurement of single top quark production in the $\mathbb{E}_{T} b \bar{b}$ final state was performed with a data set corresponding to an integrated luminosity of $2.1 \mathrm{fb}^{-1}$ [108]. This Chapter presents a new measurement using the full CDF data set. All the techniques developed in the search for s-channel single top quark production in the $E_{T} b \bar{b}$ sample presented in Chapter 6 are exploited in this update. The results of this analysis and those of the most recent $\ell v b \bar{b}$ analysis [109] are then combined to obtain a more precise measurement of the single top quark cross section and to place a more stringent limit on the CKM matrix element magnitude $\left|V_{t b}\right|$.

\section{A.1.1 Multivariate Discriminants}

All events that satisfy the kinematic and $b$-tagging criteria described in Sections 4.2.2 and 6.1.1 are considered in this analysis. A series of multivariate discriminants that take advantage of nontrivial variable correlations are employed to separate the signal from the remaining backgrounds.

To optimize the suppression of QCD multijet background, the same $\mathrm{NN}_{\mathrm{QCD}}$ multivariate discriminant that was developed in the $E_{T} b \bar{b} s$-channel single top quark search described in Section 6.1.2 is used. All events that satisfy a minimum $\mathrm{NN}_{\mathrm{QCD}}$ threshold requirement populate the signal region, in which the dominant backgrounds are from QCD multijet production, $V+$ heavy-flavour-jets events, and $t \bar{t}$ events. Events that do not meet the minimal $\mathrm{NN}_{\mathrm{QCD}}$ threshold are used to validate the background prediction with the data. From this validation, multiplicative correction factors ranging from 0.7 to 0.9 are derived for each of the 1T, TL and TT QCD multijet predictions so that the total predicted background normalizations are in agreement with data. These corrections are applied to the QCD multijet prediction in the signal region.

Two additional discriminants are developed that exploit the differences in kinematic properties between the signal and the $V$ +jets background, and the signal and $t \bar{t}$ background processes. The first discriminant $\mathrm{NN}_{V \text { jets }}$ is trained using simulated $t$-channel single top quark events for the signal sample and QCD-modeled events that satisfy the requirement on $\mathrm{NN}_{\mathrm{QCD}}$, for the background sample. The second discriminant $\mathrm{NN}_{t \bar{t}}$ is trained to separate $t$-channel single top quark from $t \bar{t}$ production, again using simulated $t$-channel single top quark events for the signal and simulated $t \bar{t}$ for the background. The values of these two discriminants are then combined in quadrature for an overall discriminant called $N N_{\text {sig }}{ }^{t}$; this is analogous to the strategy adopted to derive the final discriminant in the $\mathbb{E}_{T} b \bar{b}$ s-channel single top quark search described in Section 6.1.2. 
The s-channel optimized $\mathrm{NN}_{\text {sig }}$ discriminant as used in the $\mathbb{E}_{T} b \bar{b} s$-channel single top quark search and the $N N_{\text {sig }}{ }^{t}$ discriminant of this analysis are combined to obtain an $N N_{\text {sig }}{ }^{s+t}$ final discriminant, used to simultaneously separate both $s$ - and $t$-channel signal processes from the remaining background. For events with $\mathrm{NN}_{\text {sig }}$ output values larger than $0.6, \mathrm{NN}_{\text {sig }}$ is assigned to the $N N_{\mathrm{sig}}{ }^{s+t}$ output. For the remaining events, $N N_{\mathrm{sig}}{ }^{s+t}$ is defined as the $N N_{\mathrm{sig}^{t}}{ }^{t}$ output multiplied by 0.6. Figure A.1 shows the predicted and observed distributions of the $N_{\mathrm{sig}}{ }^{s+t}$ output variable for each of the six event subsamples used in this analysis.

\section{A.1.2 Cross Section and $\left|V_{t b}\right|$ Limit Extraction}

The sources of systematic uncertainty described in Section 6.1.3 are taken into account to measure the signal cross section.

A likelihood fit to the binned $N N_{\mathrm{sig}}{ }^{s+t}$ distribution is used to extract the single top quark signal in the presence of SM backgrounds. The Bayesian method described in Section 5.2 is employed to extract the signal cross section.

Tables A.1 and A.2 show the event yields in the two- and three-jet subsamples, respectively, as determined from applying the measurement procedure to the six discriminants shown in Figure A.1.

Table A.1 - Numbers of predicted and observed events in the two-jet signal region in the subsamples with exactly one tightly tagged jet (1T), one tightly and one loosely tagged jet (TL), and two tightly tagged jets (TT). The uncertainties in the predicted numbers of events are due to the theoretical cross section uncertainties and to the uncertainties on signal and background modeling. Both the uncertainties and the central values are those determined by the fit to the data with theory constraints.

\begin{tabular}{cccc}
\hline \hline Category & $1 \mathrm{~T}$ & $\mathrm{TL}$ & $\mathrm{TT}$ \\
\hline$t \bar{t}$ & $243 \pm 24$ & $84.8 \pm 9.3$ & $92.4 \pm 8.4$ \\
Diboson & $285 \pm 26$ & $51.3 \pm 4.6$ & $37.2 \pm 3.4$ \\
$V H$ & $12.6 \pm 1.4$ & $6.6 \pm 0.8$ & $7.6 \pm 0.8$ \\
$V+$ jets & $6528 \pm 2048$ & $694 \pm 216$ & $220 \pm 69$ \\
MJ & $8322 \pm 180$ & $928 \pm 59$ & $300 \pm 32$ \\
\hline$s$-ch single top & $86.2 \pm 47.7$ & $41.8 \pm 23.2$ & $45.9 \pm 25.3$ \\
$t$-ch single top & $161 \pm 31$ & $10.8 \pm 2.1$ & $9.2 \pm 1.7$ \\
\hline Total prediction & $15643 \pm 2057$ & $1775 \pm 225$ & $709 \pm 80$ \\
\hline Observed & 15312 & 1743 & 686 \\
\hline \hline
\end{tabular}

The observed single top quark production cross section $\left(\sigma_{\mathrm{obs}}^{s+t}\right)$ is $3.53_{-1.16}^{+1.25} \mathrm{pb}$, consistent with the SM prediction of $3.15 \pm 0.36 \mathrm{pb}$ [22]. The magnitude of $V_{t b}$ is extracted from the single top quark cross section posterior probability density by the relation $\left|V_{t b}\right|_{\mathrm{obs}}^{2}=$ $\left|V_{t b}\right|_{\mathrm{SM}}^{2} \sigma_{\mathrm{obs}}^{s+t} / \sigma_{\mathrm{SM}}^{s+t}$, where variables with the subscript "SM" ("obs") correspond to the the- 

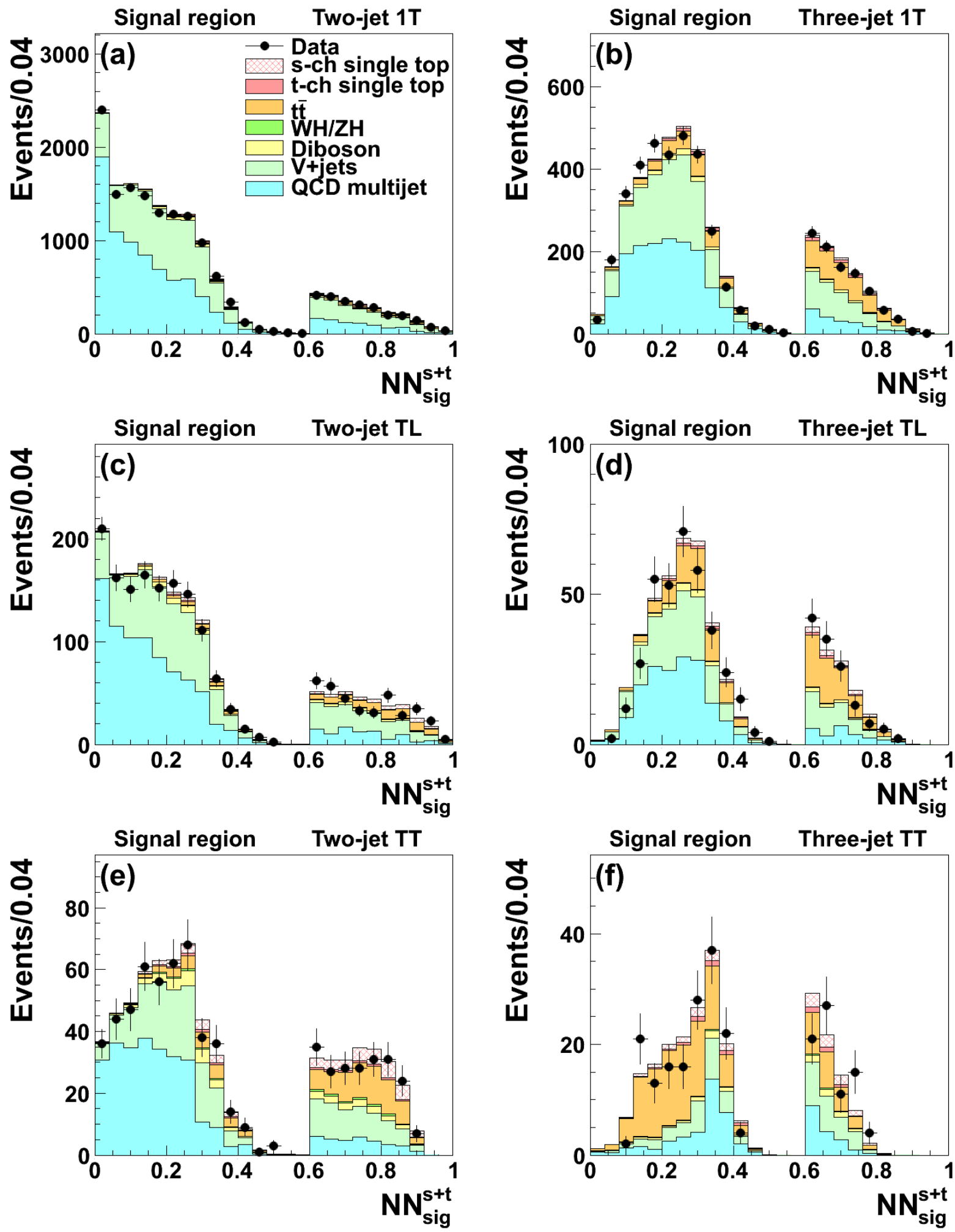

Figure A.1 - Predicted and observed $N N_{\text {sig }}{ }^{s+t}$ distributions in the signal region, for 1T two-jet (a), 1T three-jet (b), TL two-jet (c), TL three-jet (d), TT two-jet (e) and TT three-jet (f) subsamples.

oretical predictions (observed values) [9]. We assume $\left|V_{t b}\right|_{S M}^{2}$ is unity and fix the $s$ - and $t$-channel relative contributions to their SM prediction. Including the theoretical uncer- 
Table A.2 - Numbers of predicted and observed three-jet events in the 1T, TL, and TT subsamples.

\begin{tabular}{cccc}
\hline \hline Category & 1T & TL & TT \\
\hline$t \bar{t}$ & $597 \pm 60$ & $118 \pm 13$ & $110 \pm 10$ \\
Diboson & $108 \pm 10$ & $15.7 \pm 1.5$ & $8.8 \pm 0.8$ \\
$V H$ & $6.0 \pm 0.7$ & $1.9 \pm 0.2$ & $2.2 \pm 0.2$ \\
$V+$ jets & $1610 \pm 505$ & $165 \pm 51$ & $50 \pm 16$ \\
MJ & $1818 \pm 49$ & $188 \pm 15$ & $55.9 \pm 7.6$ \\
\hline$s$-ch single top & $45.7 \pm 25.3$ & $15.4 \pm 8.5$ & $16.2 \pm 8.9$ \\
$t$-ch single top & $82.2 \pm 15.8$ & $7.5 \pm 1.5$ & $6.8 \pm 1.3$ \\
\hline Total prediction & $4266 \pm 512$ & $510 \pm 56$ & $250 \pm 22$ \\
\hline Observed & 4198 & 490 & 237 \\
\hline \hline
\end{tabular}

tainty of the signal cross section (5.8\% for s-channel, $6.2 \%$ for $t$-channel) [22] and assuming a uniform prior in the interval $0<\left|V_{t b}\right|^{2}<1$, a lower bound on $\left|V_{t b}\right|$ of 0.63 is obtained at the $95 \%$ C.L., as shown in Figure A.2.

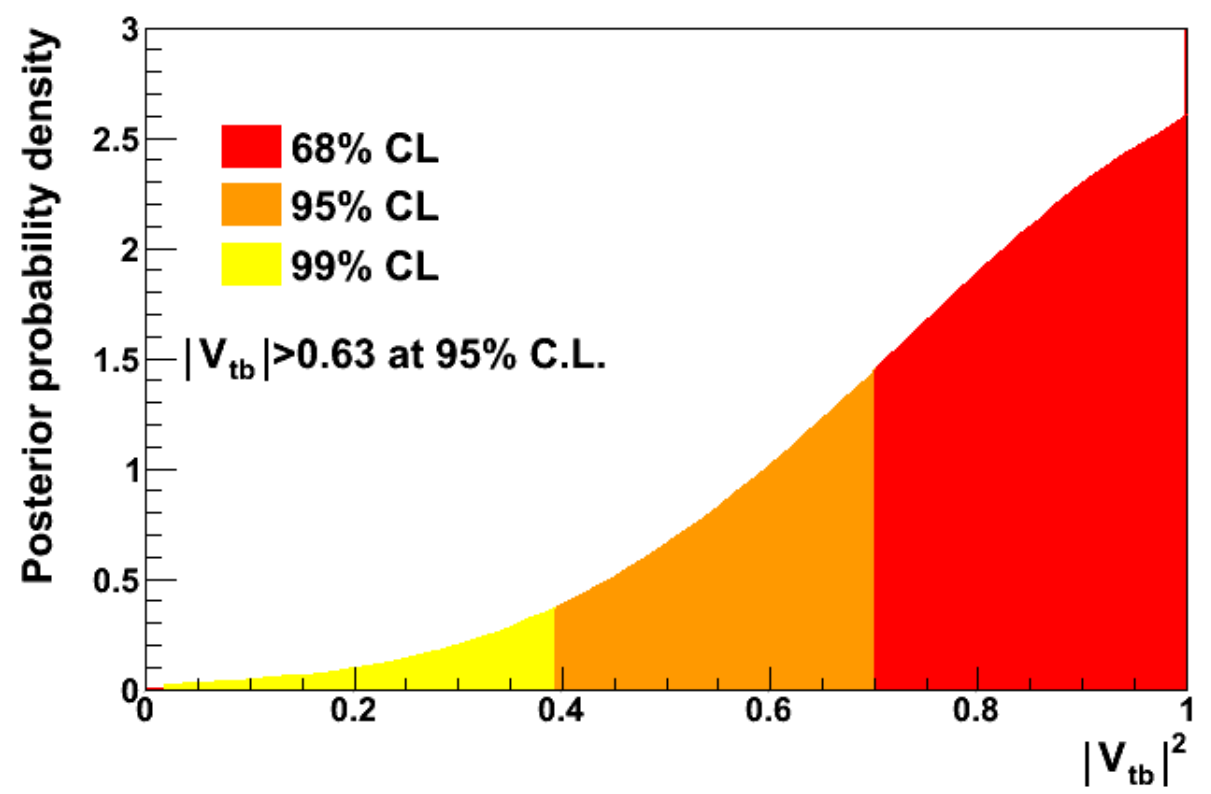

Figure A.2 - The posterior probability distribution for the for the $\mathbb{E}_{T} b \bar{b}$ analysis presented here. The orange region indicates the allowed values of $\left|V_{t b}\right|^{2}$ corresponding to the limit of $\left|V_{t b}\right|>0.63$ at $95 \%$ C.L.

We measure the $t$-channel cross section by itself to be $1.19_{-0.97}^{+0.93} \mathrm{pb}$, where the s-channel cross section is constrained to its SM prediction; this result is consistent with the SM prediction of $2.10 \pm 0.12 \mathrm{pb}$ [22]. We also compute the two-dimensional posterior for the $s$ - and $t$-channel cross sections, where the relative contributions of both channels are allowed to vary independently; the result is shown in Figure A.3. 


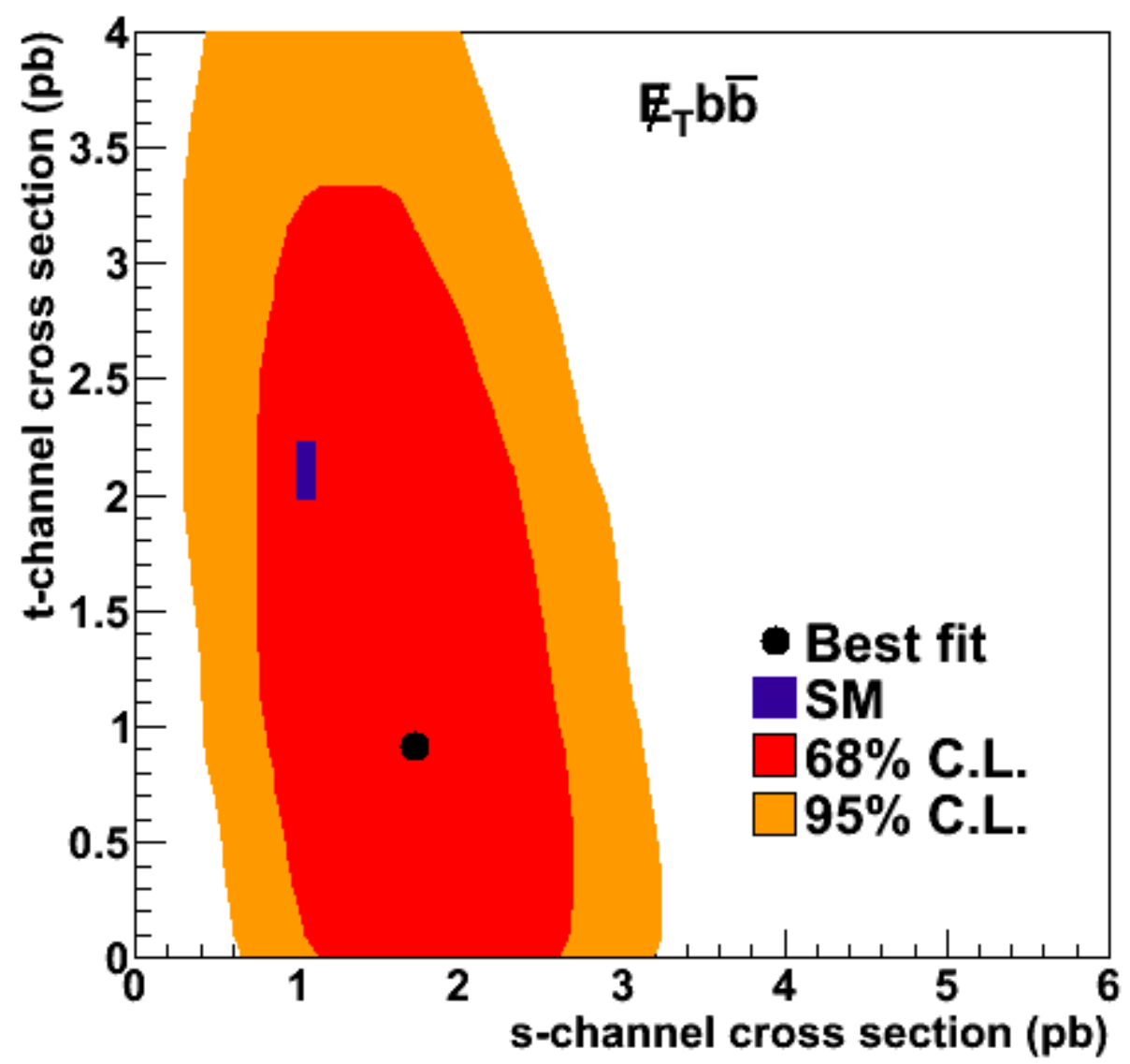

Figure A.3 - Two-dimensional posterior probability densities of the $s$ - and $t$-channel cross sections for the $\ddot{E}_{T} b \bar{b}$ analysis presented here.

\section{A.2 CDF Combination}

These results are combined with those of the most recent CDF measurement of single top quark production in the $\ell v b \bar{b}$ sample [109], which measured a cross section of $3.04_{-0.53}^{+0.57} \mathrm{pb}$ assuming a top quark mass of $172.5 \mathrm{GeV} / \mathrm{c}^{2}$. The combination is achieved following the same procedure explained in Section 5.1.2 and applied in Section 6.2. In the $\ell v b \bar{b}$ analysis, candidate events were selected by requiring exactly one reconstructed charged lepton ( $e$ or $\mu$ ) in the final state. Hence, no such events are included in the $\mathbb{E}_{T} b \bar{b}$ analysis described above. The uncertainties associated with the theoretical cross sections of the $t \bar{t}, V V$, and $V H$ production processes, and those associated with the luminosity are taken as fully correlated between the two analyses.

The combined measurement results in a single top quark production cross section of $3.02_{-0.48}^{+0.49} \mathrm{pb}$, consistent with the SM prediction. From the posterior probability density on $\left|V_{t b}\right|^{2}$, a $95 \%$ C.L. lower limit of $\left|V_{t b}\right|>0.84$ is obtained, as shown in Figure A.4. 


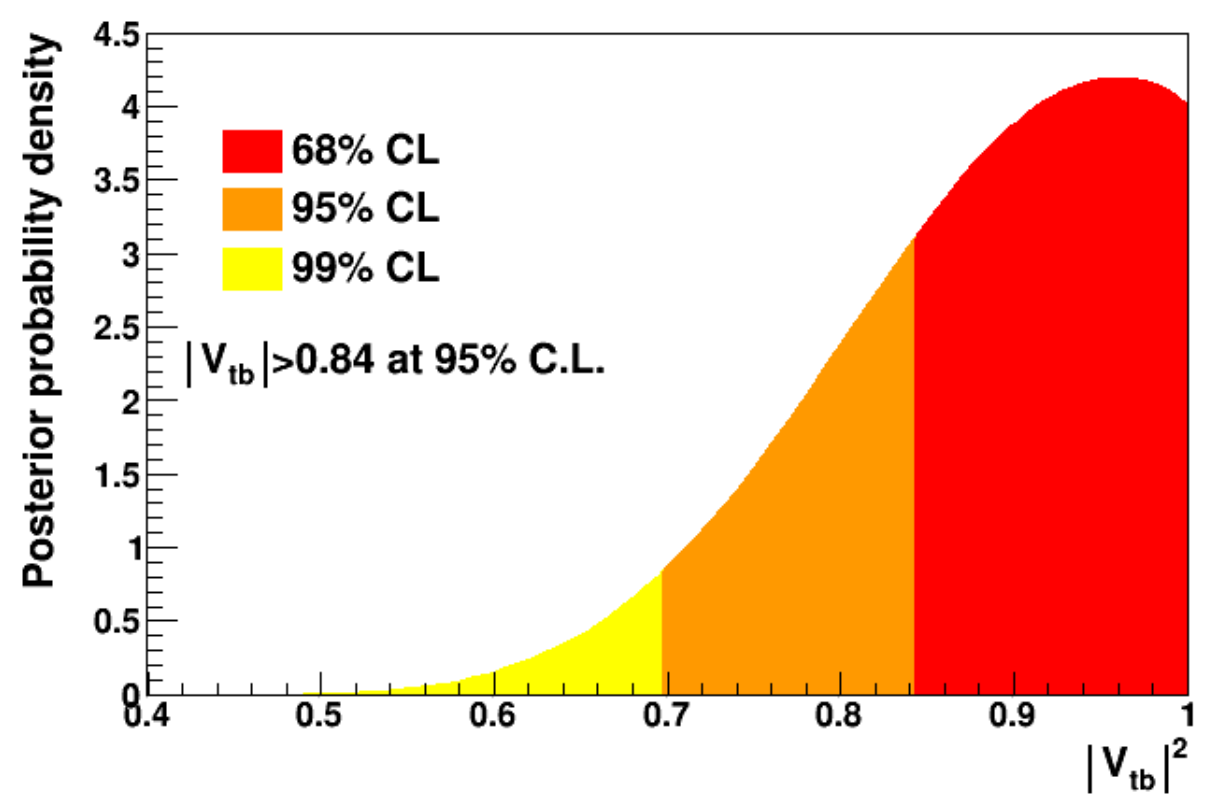

Figure A.4 - The posterior probability distribution for the CDF combination of the $E_{T} b \bar{b}$ and $\ell v b \bar{b}$ analysis results. The orange region indicates the allowed values of $\left|V_{t b}\right|^{2}$ corresponding to the limit of $\left|V_{t b}\right|>0.84$ at $95 \%$ C.L.

The $t$-channel cross section, measured in the same way as for the $E_{T} b \bar{b}$ analysis, is $1.65_{-0.36}^{+0.38}$ $\mathrm{pb}$, in agreement with the SM prediction given above. The two-dimensional posterior probability is shown in Figure A.5, where the relative $s$ - and $t$-channel contributions are allowed to vary freely.

\section{A.3 Tevatron Combination}

The CDF result is then combined with the result of the analysis performed by the D0 Collaboration [98]. The D0 analysis is described in Section 6.3. At D0, multivariate discriminants were optimized to separate signal events from their large background contributions in each of the analysis subsamples [98]. This is carried out separately considering either $s$-channel single top quark production as the only signal, or $t$-channel single top quark production as the only signal. These two discriminants are used simultaneously in a joint discriminant, sensitive to both $s$ - and $t$-channel signals, to compare data to the modeled distributions for each contributing signal and background process.

The combined Tevatron cross section measurement is obtained using a Bayesian statistical analysis (see Sections 5.1.2, 5.2) .

The sources of systematic uncertainties for the combined $t$ and $s+t$-channels are identical to those used in Section 6.3, summarized in Table 6.3. There it is also given which 


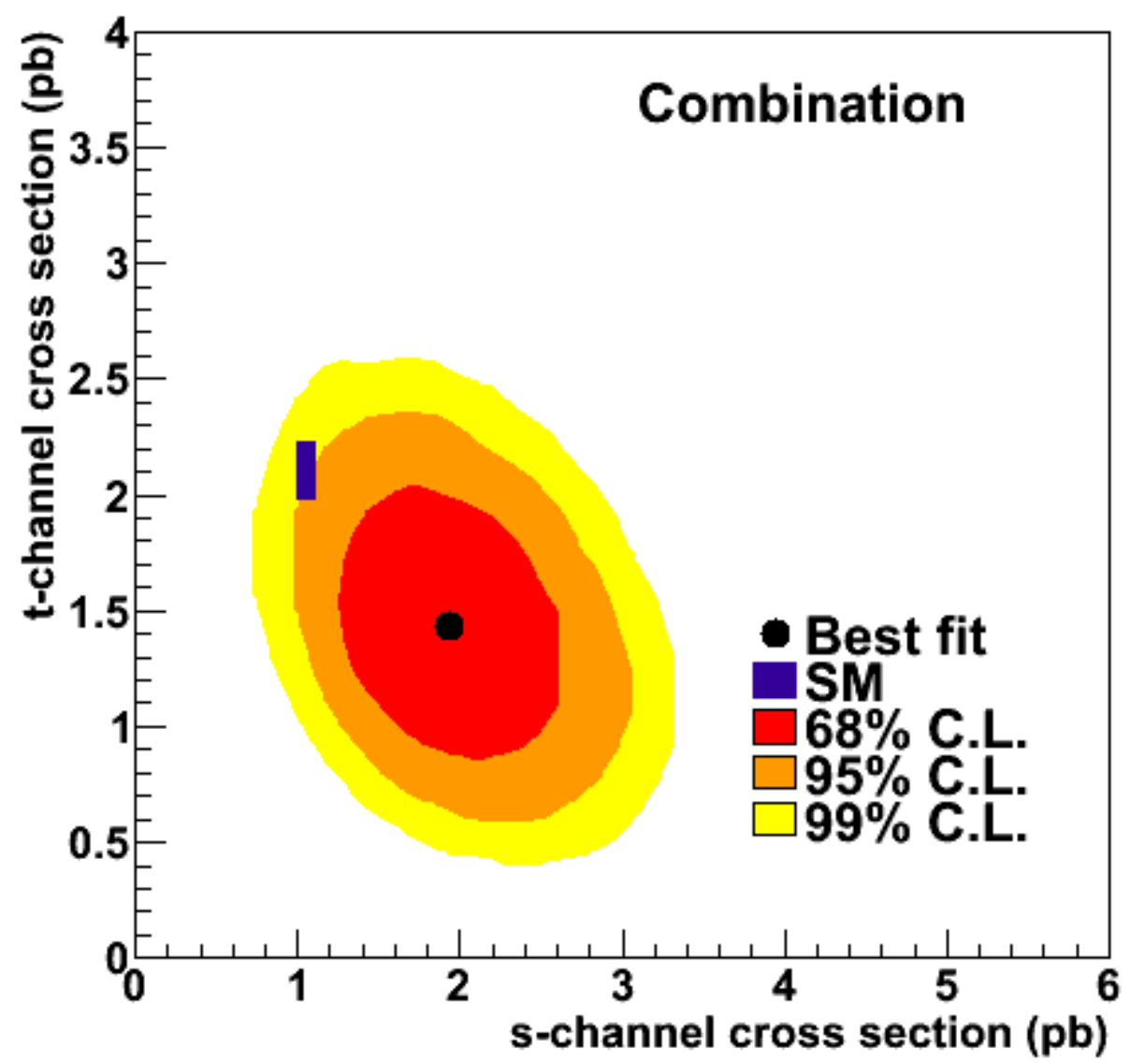

Figure A.5 - Two-dimensional posterior probability densities of the $s$ - and $t$-channel cross sections for the CDF combination of the $\mathscr{E}_{T} b \bar{b}$ and $\ell v b \bar{b}$ analysis results.

sources of systematic uncertainty common to measurements of both collaborations are assumed to be $100 \%$ correlated, and which uncertainties are assumed to be uncorrelated. The dependence of the results on their correlation is found to be negligible.

A two-dimensional (2D) posterior probability density is constructed as a function of $\sigma_{\mathcal{S}}$ and $\sigma_{t}$ in analogy to the one-dimensional (1D) posterior probability described in Section 6.3. The measured cross section is quoted as the value at the position of the maximum, and the area of the distribution in the most narrow region that encompasses $68 \%$ of the entire area defines the uncertainty (statistical and systematic components combined). The expected cross sections are obtained by setting the number of events in each channel equal to the value given by the prediction of the sum of the SM signal and background.

Figure A.6 shows the expected $s$ and $t$-channel contributions and the data together with the background prediction.

The extracted 2D posterior probability distribution as a function of $\sigma_{t}$ and $\sigma_{s}$ is presented in Figure A.7. To measure the mean value and uncertainty on the individual cross sec- 


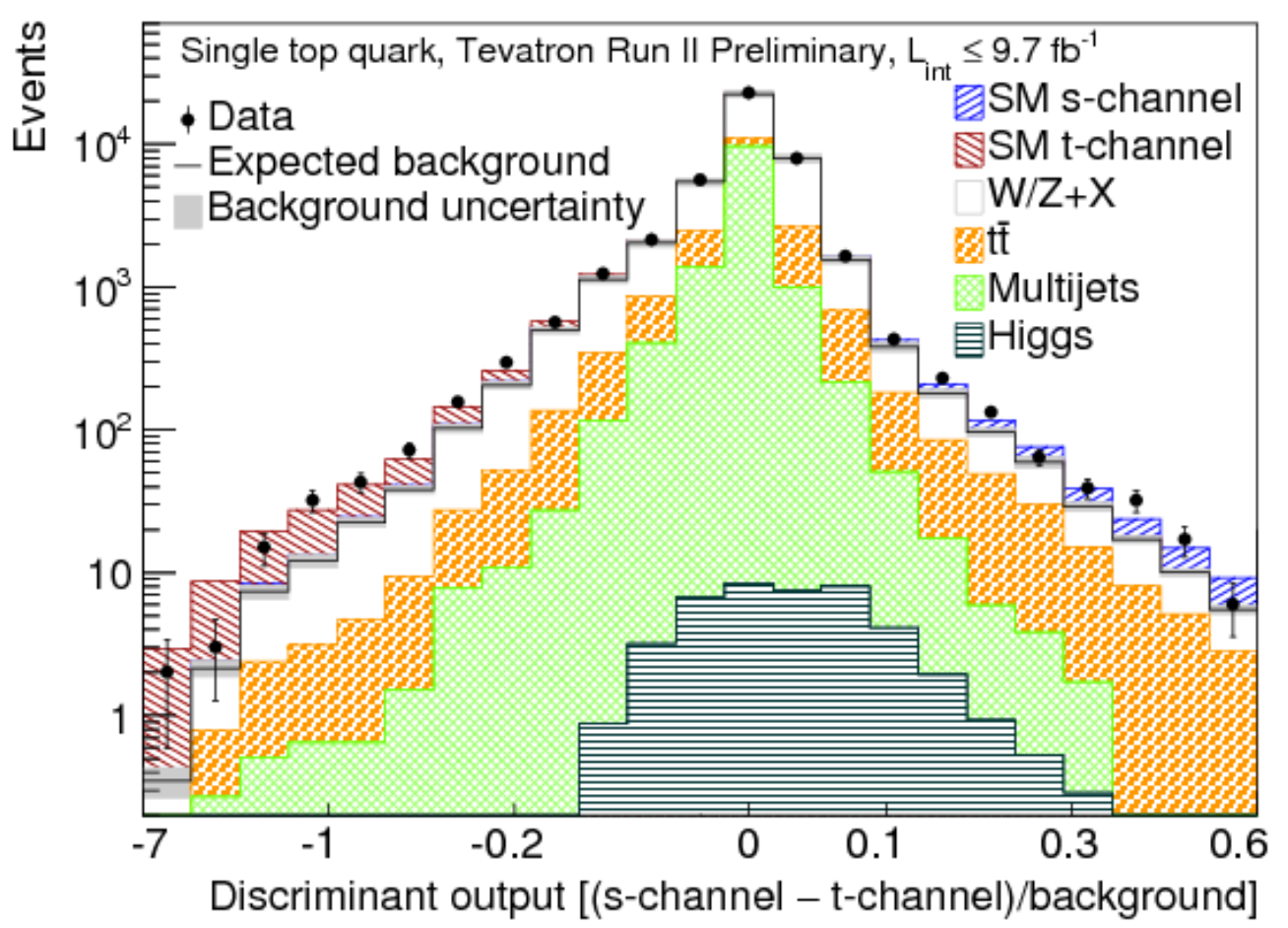

Figure A.6 - Distribution of the discriminant, summed over bins with similar ratios of (schannel $-t$-channel) signals over background. Displayed are the data, $s$ - and $t$-channel production as predicted in the SM and the expected background. The total expected background (black solid line) is shown with uncertainty (gray shaded band). A nonlinear scale is used on the abscissa to better display the range of values.

tions, the $1 \mathrm{D}$ posterior probability functions are obtained by integrating the 2D posterior over the other variable. The most probable value for the combined $t$-channel cross section is $\sigma_{t}=2.25_{-0.31}^{+0.29} \mathrm{pb}$ for a top-quark mass of $172.5 \mathrm{GeV}$. The combined $s+t$ cross section $\sigma_{s+t}$ is measured without assuming the SM ratio of $\sigma_{s} / \sigma_{t}$ by forming first a $2 \mathrm{D}$ posterior of $\sigma_{s+t}$ versus $\sigma_{t}$ and then integrating over all possible values of $\sigma_{t}$ to derive the $1 \mathrm{D}$ estimate of $\sigma_{s+t}$. The combined cross section is $\sigma_{s+t}=3.30_{-0.40}^{+0.52} \mathrm{pb}$ for a top-quark mass of $172.5 \mathrm{GeV}$. The systematic uncertainty on the measured cross section due to the uncertainty on the top quark mass is negligible compared with the other uncertainties [78]. Figure A.8 gives a graphical presentation of the individual and combined measurements including previous measurements of the s-channel cross section (Section 6.3). All measurements agree within their uncertainties with the SM prediction [22].

Using the same multivariate discriminants for $s$ and $t$ channels of the different analyses, a Bayesian posterior probability density was formed for $\left|V_{t b}\right|^{2}$ assuming a uniform prior, without assumptions on the $\sigma_{s} / \sigma_{t}$ production ratio. Additional theoretical uncertainties are considered for the $s-$ and $t$-channel cross sections [22]. The extracted posterior probability distribution for $\left|V_{t b}\right|^{2}$ is presented in Figure A.9. A value of $\left|V_{t b}\right|=1.02_{-0.05}^{+0.06}$ is obtained. Restricting the prior to the SM region [0,1], a limit of $\left|V_{t b}\right|>0.92$ is extracted at 95\% C.L.. 


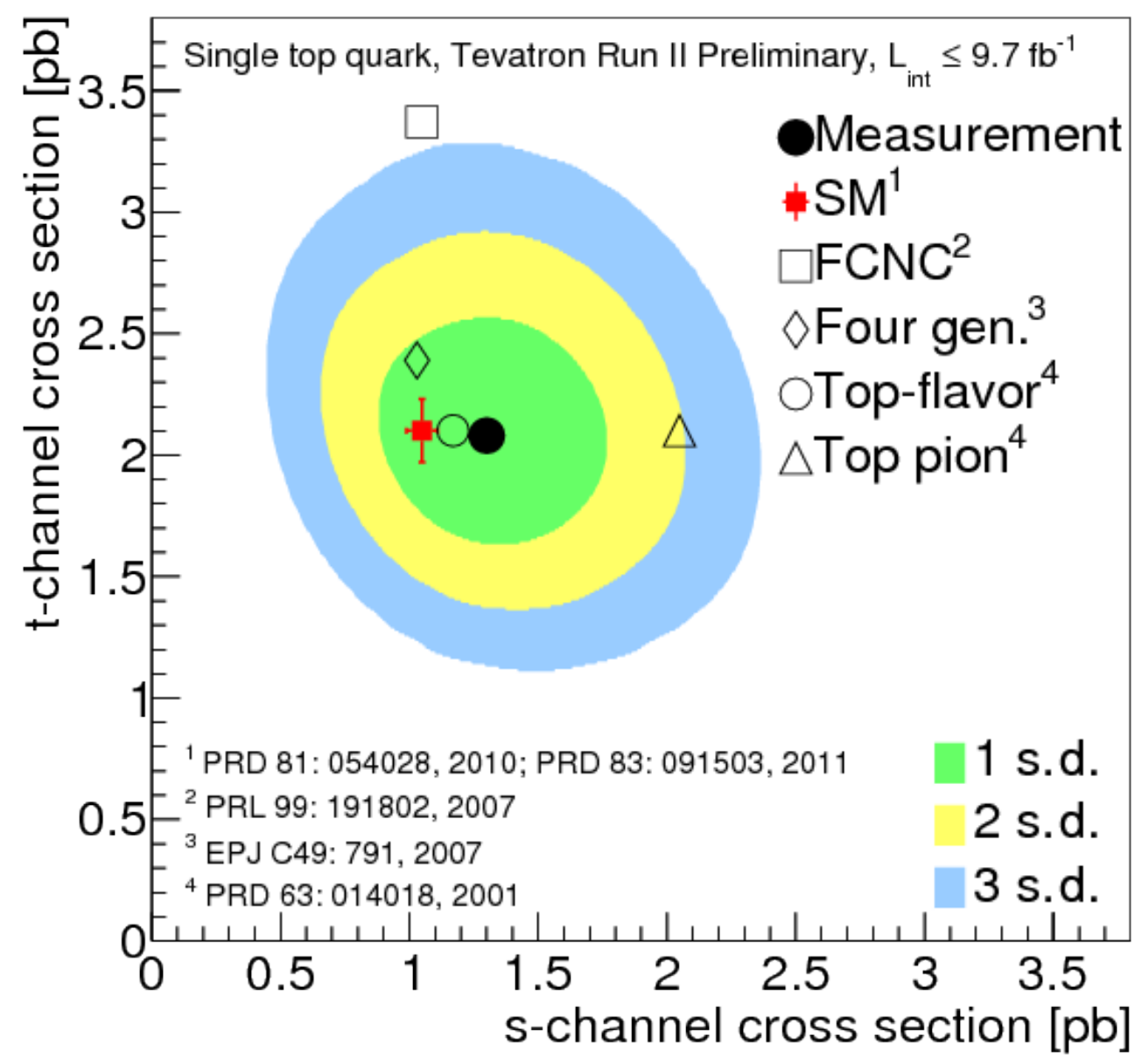

Figure A.7 - The 2D posterior probability with one, two, and three standard deviation probability contours for the combination of the CDF and D0 analysis channels compared with the theoretical prediction of the SM [22]. Several BSM predictions are also shown. 
Tevatron Run II Preliminary single top quark summary Measurement s-channel: CDF

PRL 112, 231805 (2014)

D0

PLB 726, 656 (2013)

Tevatron

PRL 112, 231803 (2014)

t-channel:

CDF

arXiv:1410.4909

D0

PLB 726, 656 (2013)

Tevatron

$\mathrm{s}+\mathrm{t}$ :

CDF

arXiv:1410.4909

D0

PLB 726, 656 (2013)

Tevatron

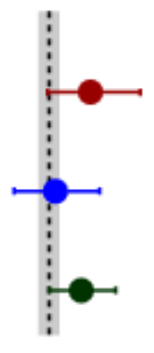

Cross section $[\mathrm{pb}]$

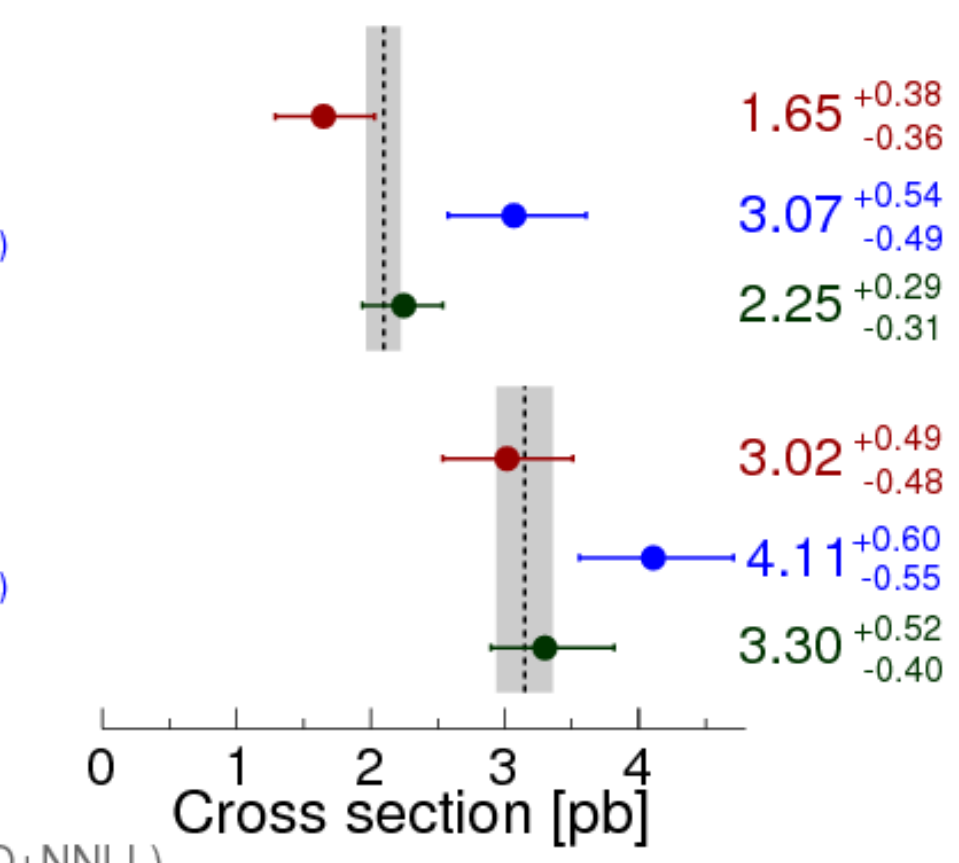

Theory (NLO+NNLL)

PRD81 054028 (2010), PRD83 091503 (2011)

$$
\mathrm{m}_{\mathrm{t}}=172.5 \mathrm{GeV}
$$

Figure A.8 - Measured single-top-quark production cross sections from the CDF and D0 Collaborations in different production channels and the Tevatron combinations of these analyses compared with the NLO+NNLL theoretical prediction [22]. 


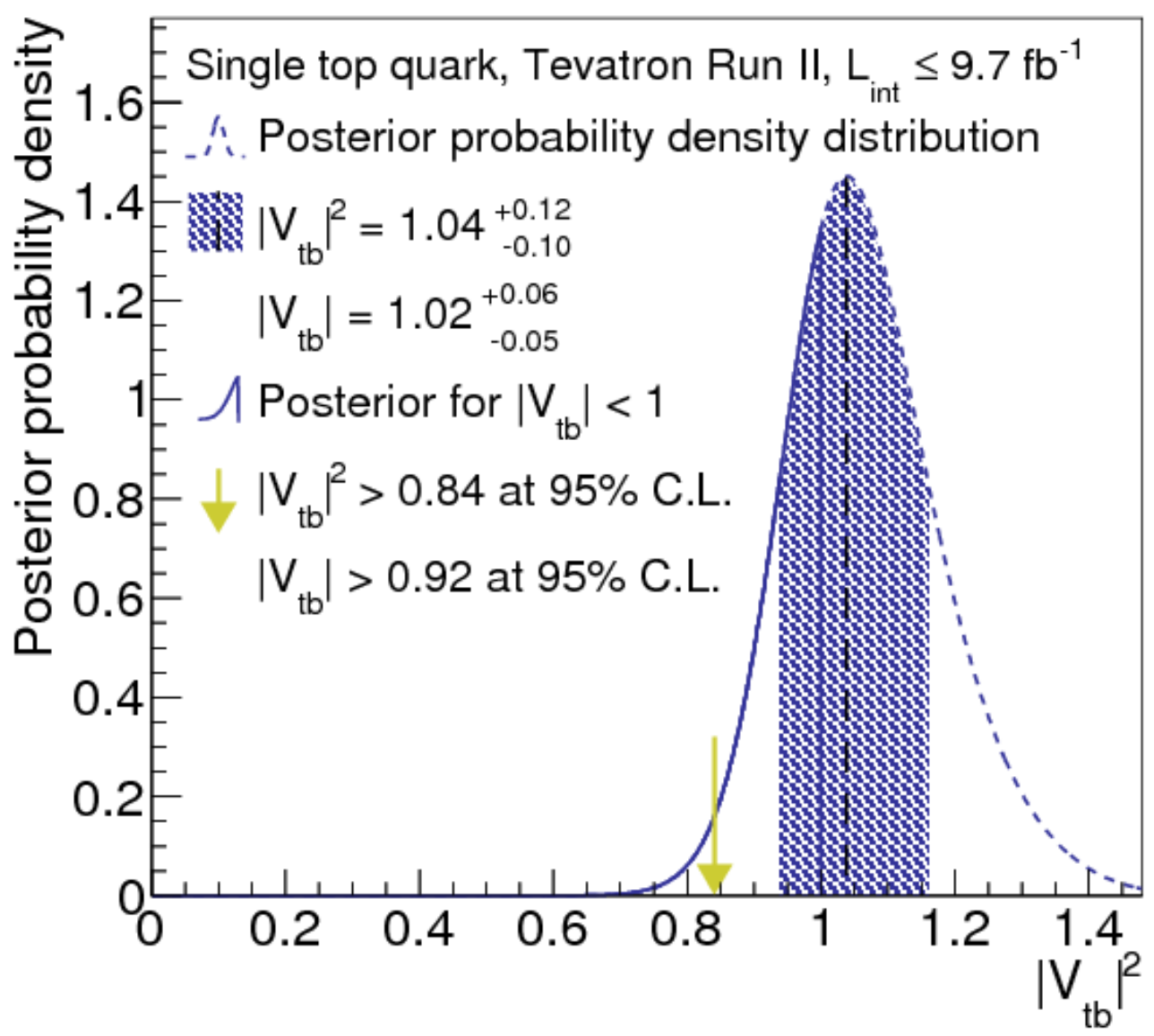

Figure A.9 - The posterior probability distribution for the combination of CDF and D0 analysis channels. The region delimited by the solid blue line indicates the allowed values of $\left|V_{t b}\right|^{2}$ corresponding to the limit of $\left|V_{t b}\right|>0.92$ at $95 \%$ C.L. 


\section{Appendix B}

\section{Validation Plots for the Single Top Analyses}

In this Section, validation plots are provided for the single top analyses. The predicted and the observed distributions for a number of kinematic variables, including those used as inputs to the $\mathrm{NN}_{\mathrm{QCD}}, \mathrm{NN}_{V \text { jets, }}$ and $\mathrm{NN}_{t \bar{t}}$ discriminants, are tested in the EWK and QCD control samples defined in Sections 4.2.2 and 6.1.2, respectively. Plots for the most separating variables, as well as for the neural network output values, are shown in this Section. The agreement between the model and the data distribution is satisfying.

\section{B.1 Two-Jets EWK Sample}
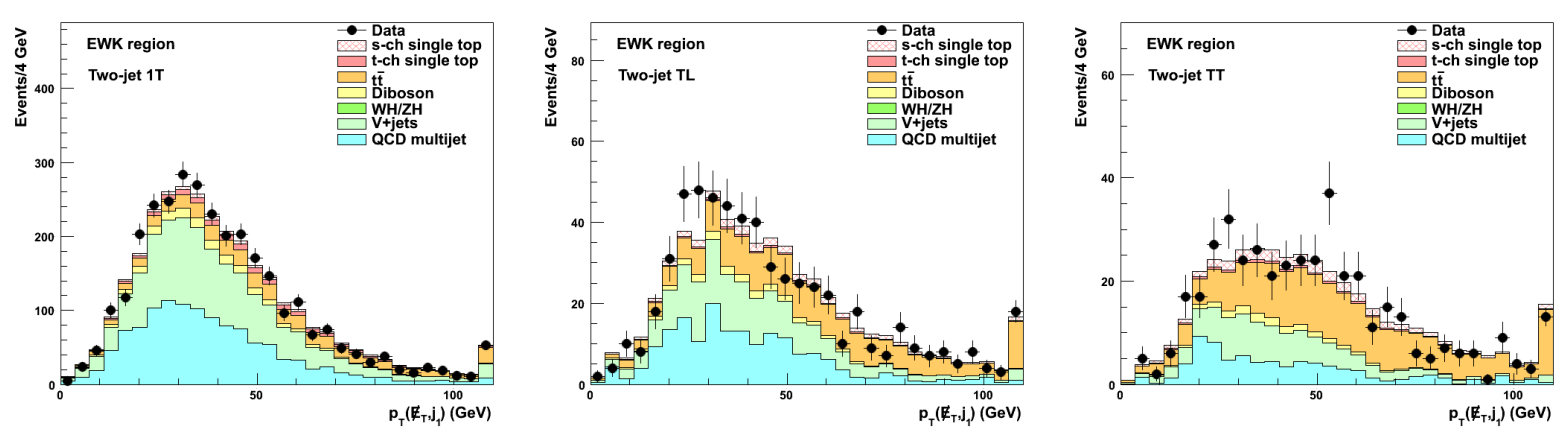

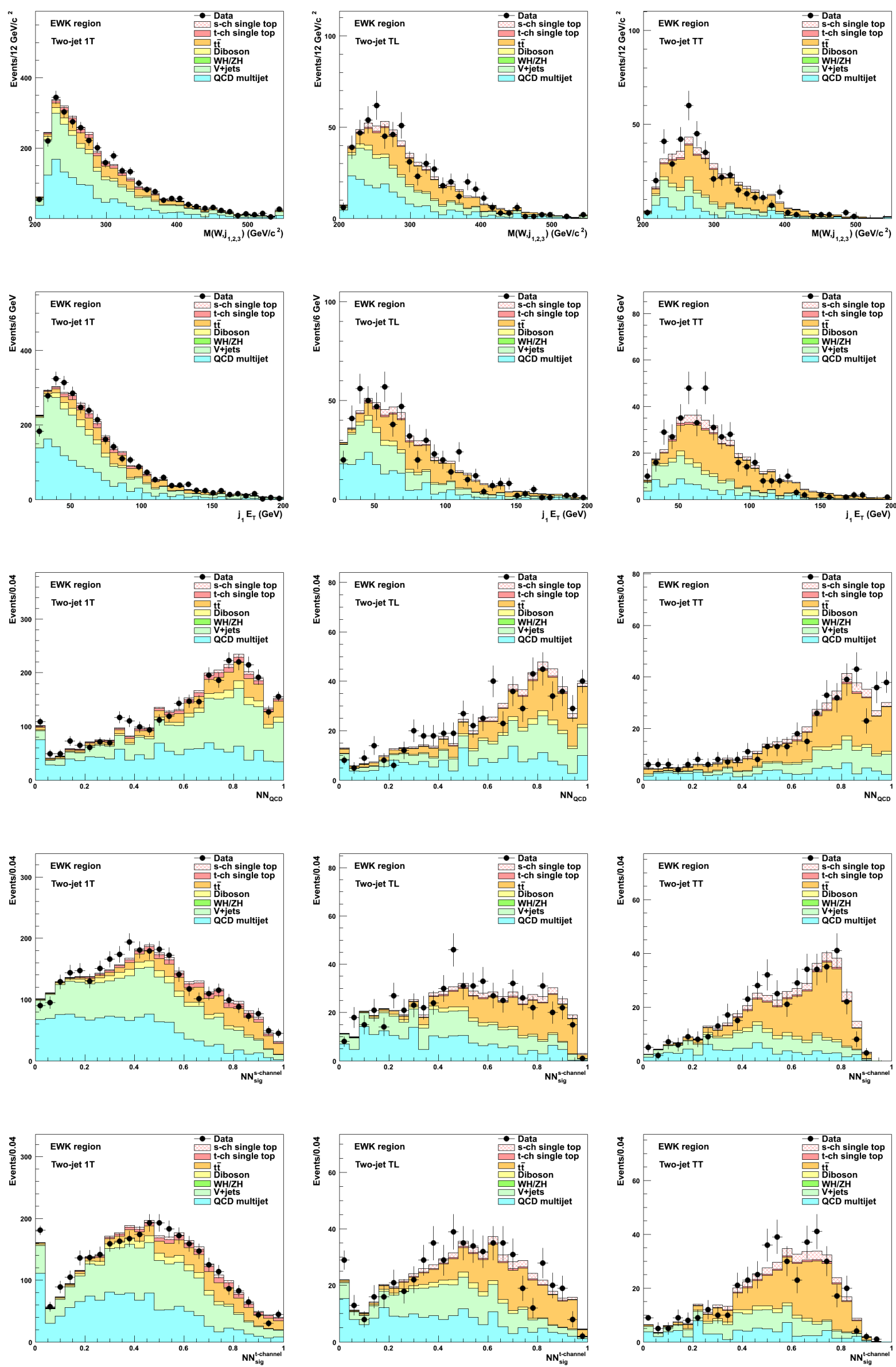

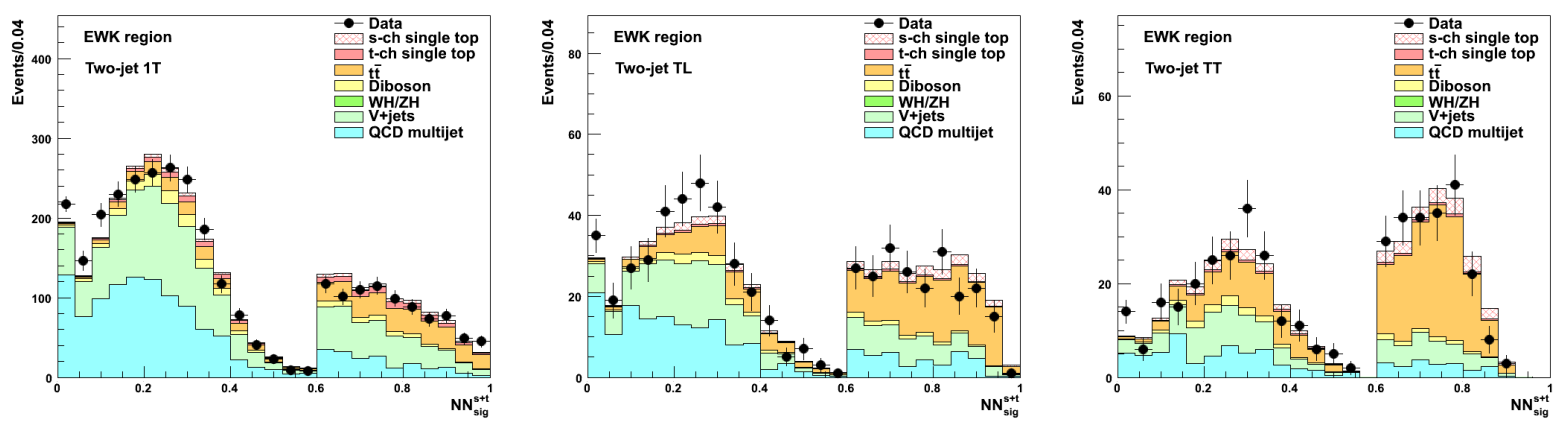


\section{B.2 Three-Jets EWK Sample}
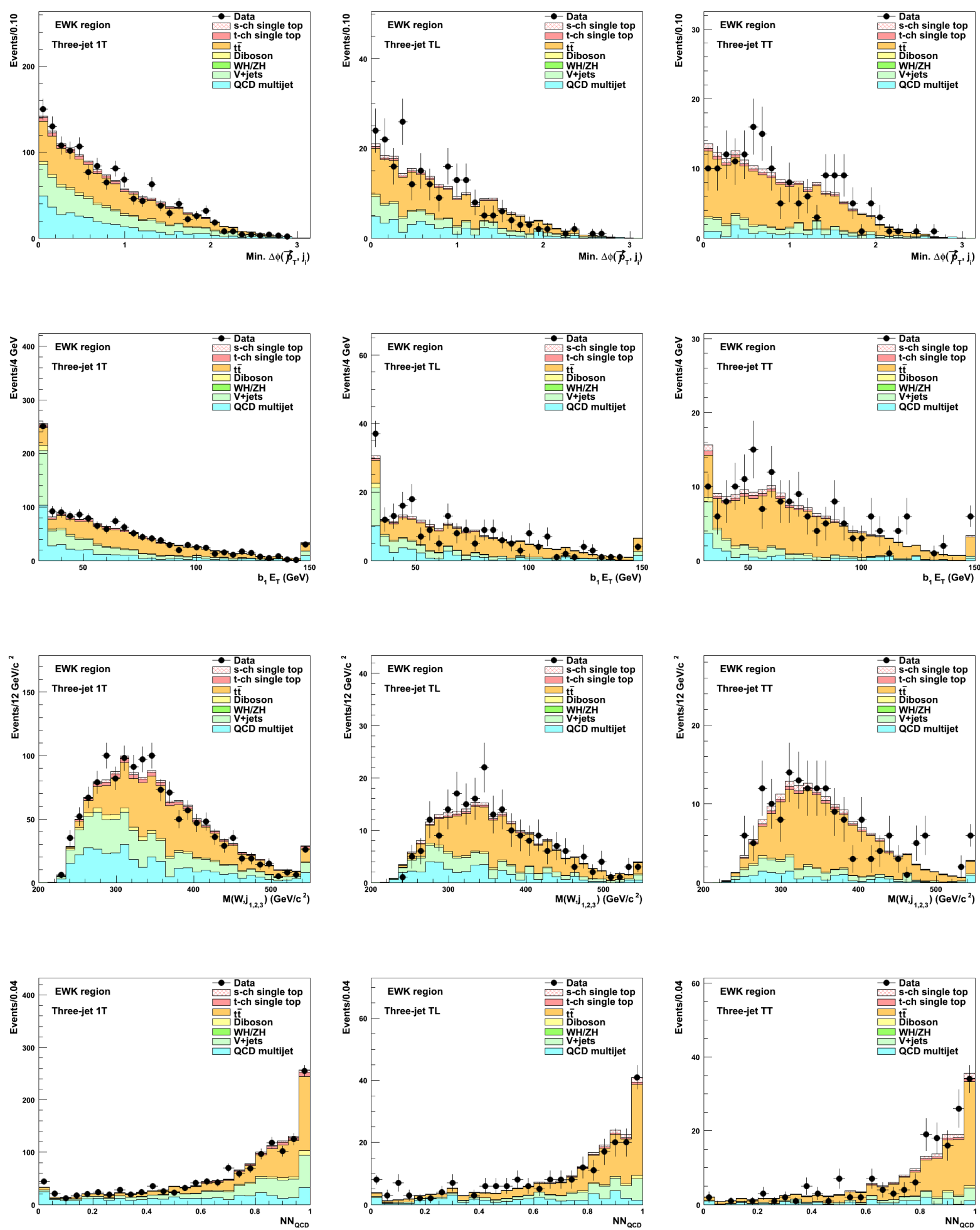

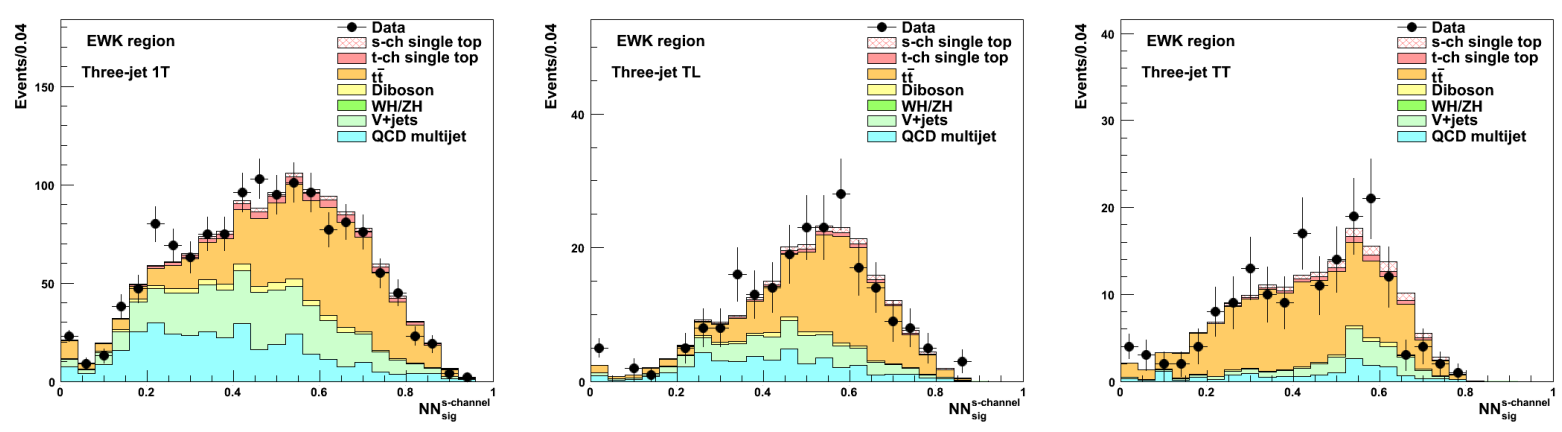


\section{B.3 Two-Jets QCD Sample}
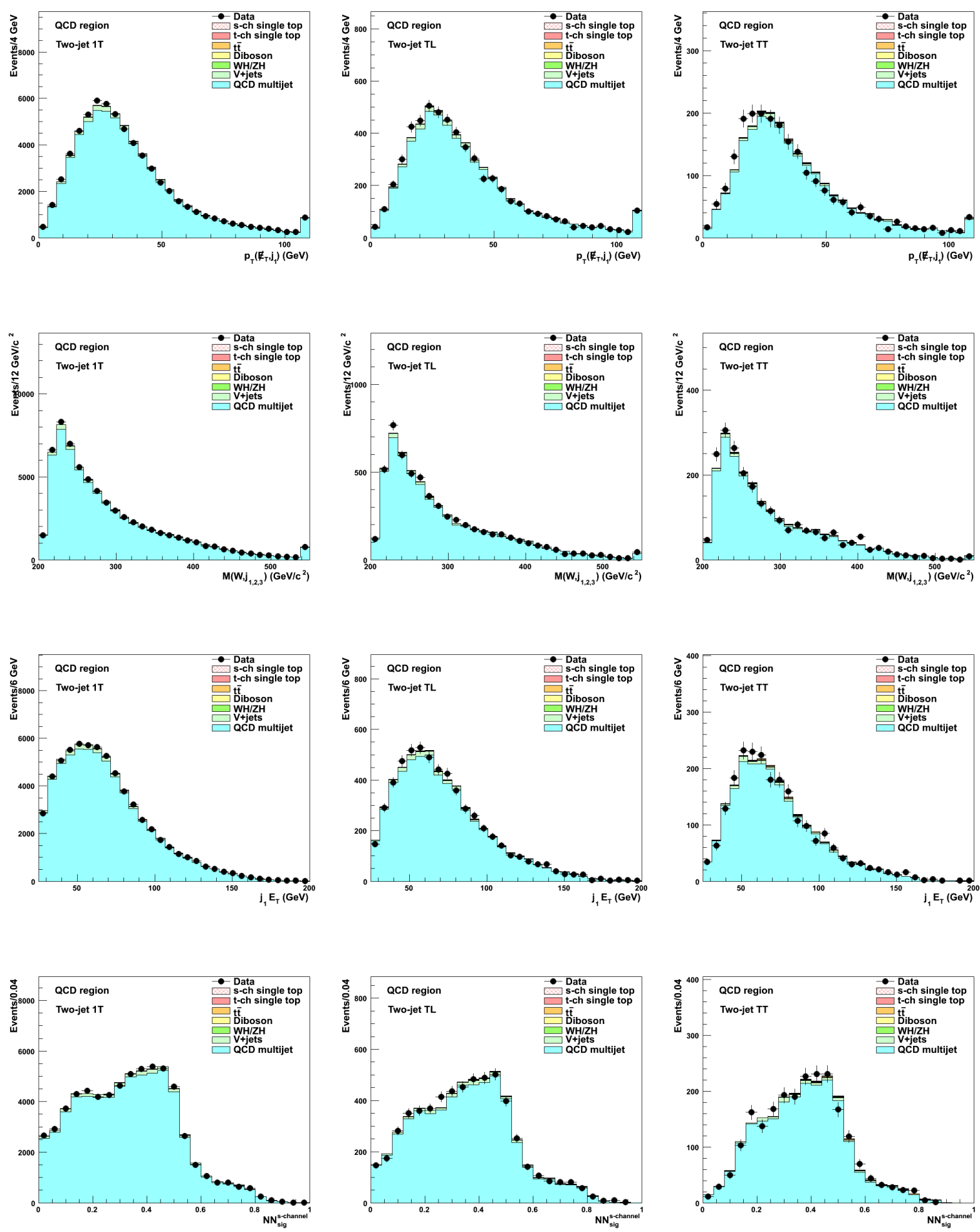

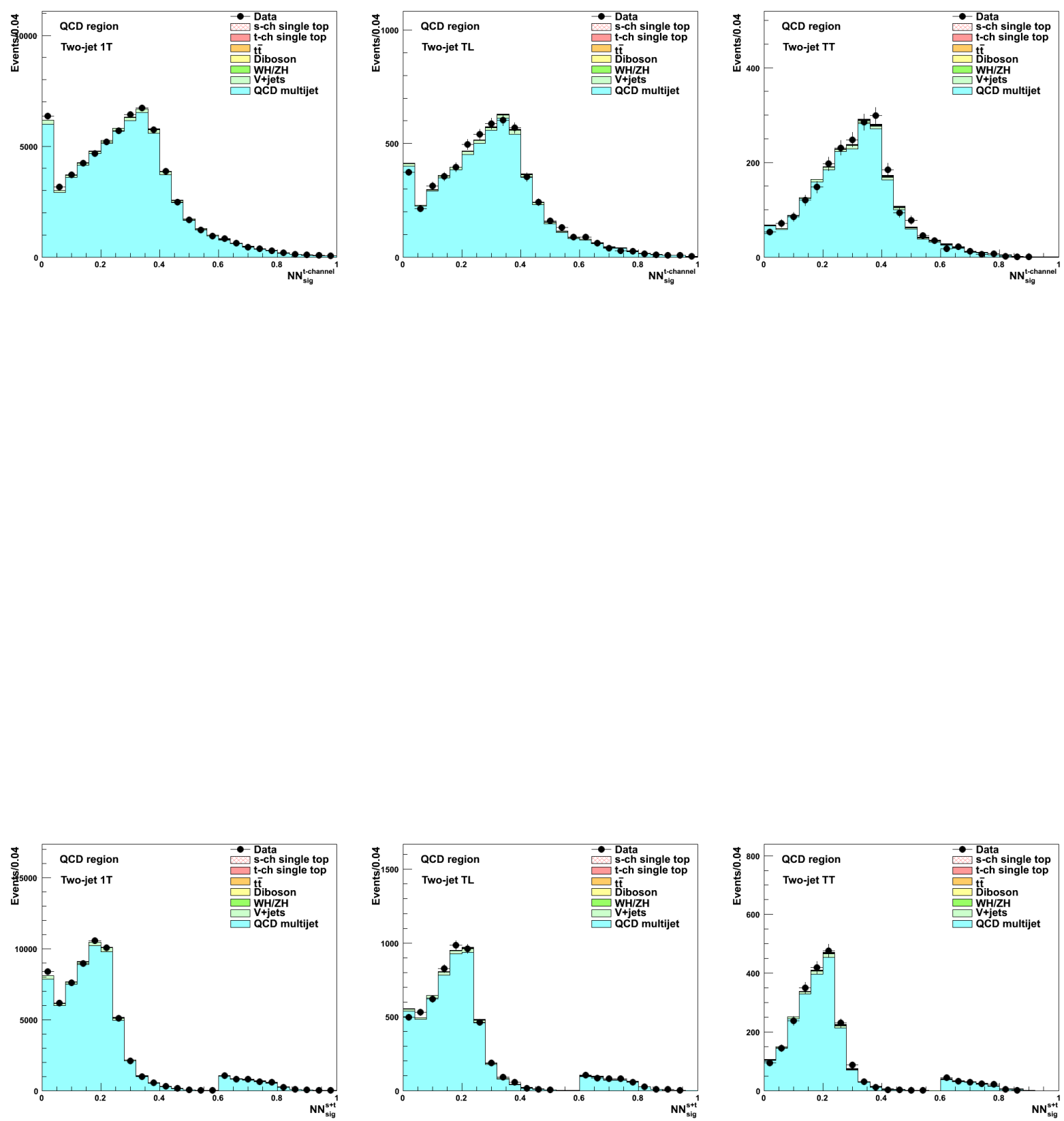


\section{B.4 Three-Jets QCD Sample}
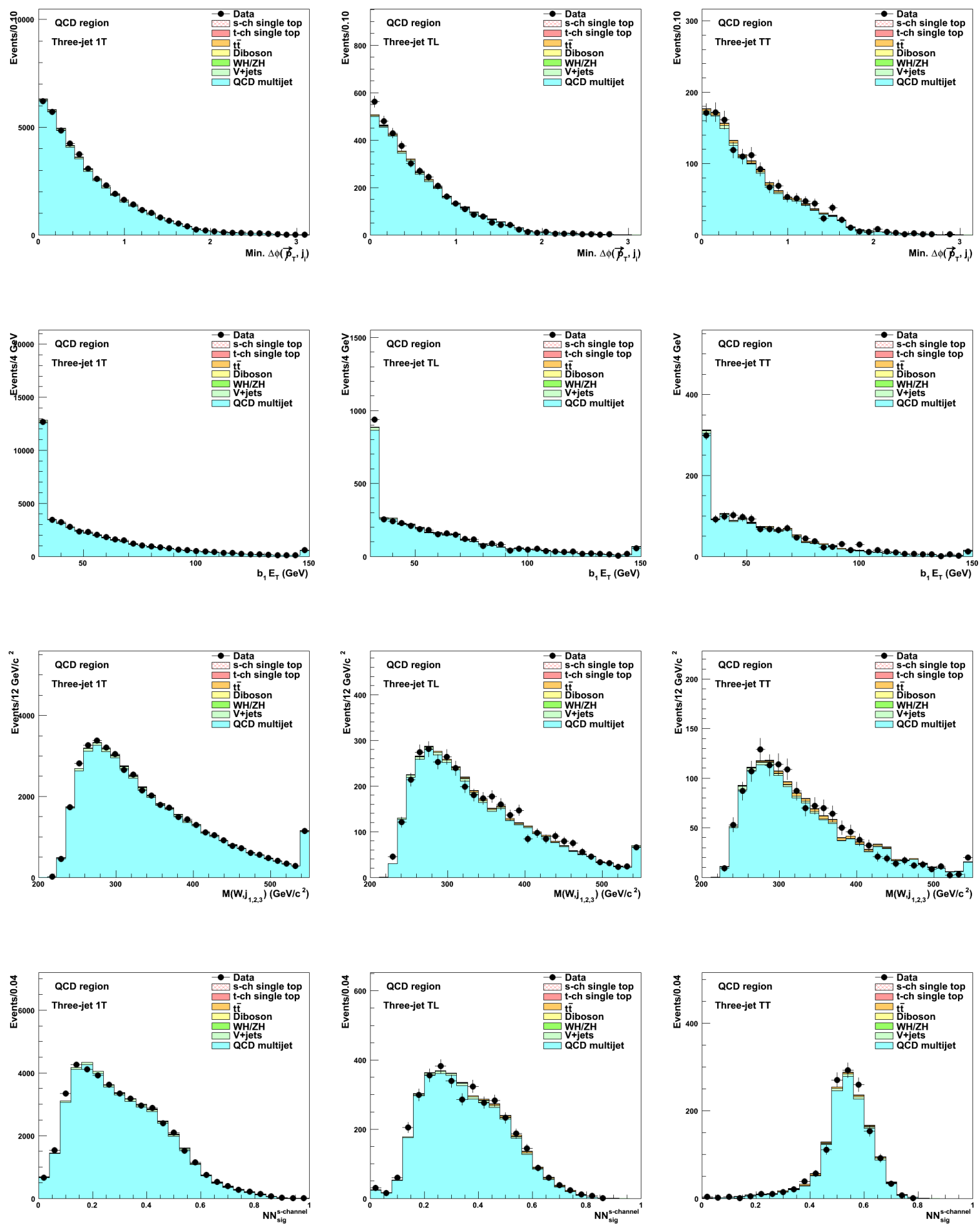

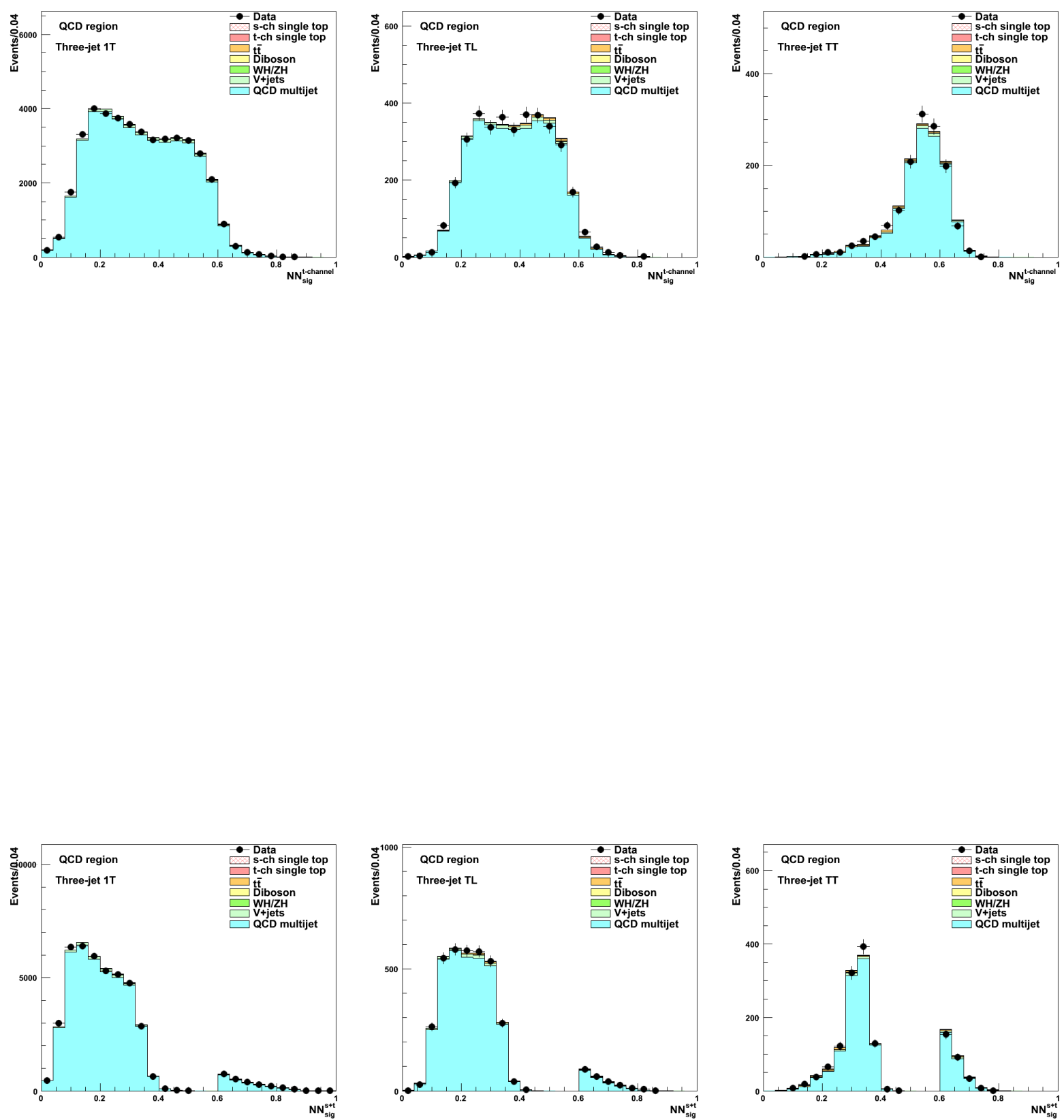


\section{Appendix C}

\section{Validation Plots for the $W^{\prime}$ Boson Analysis}

In this Section, validation plots are provided for the $W^{\prime}$ boson analysis. The predicted and the observed distributions for a number of kinematic variables, including those used as inputs to the $\mathrm{NN}_{\mathrm{QCD}}, \mathrm{NN}_{V \text { jets, }}$ and $\mathrm{NN}_{t \bar{t}}$ discriminants, are tested in the QCD control sample defined in Section 7.2. Plots for the most separating variables, as well as for the neural network output values, are shown in this Section. The agreement between the model and the data distribution is satisfying.

\section{C.1 Two-Jets Lepton QCD Sample}
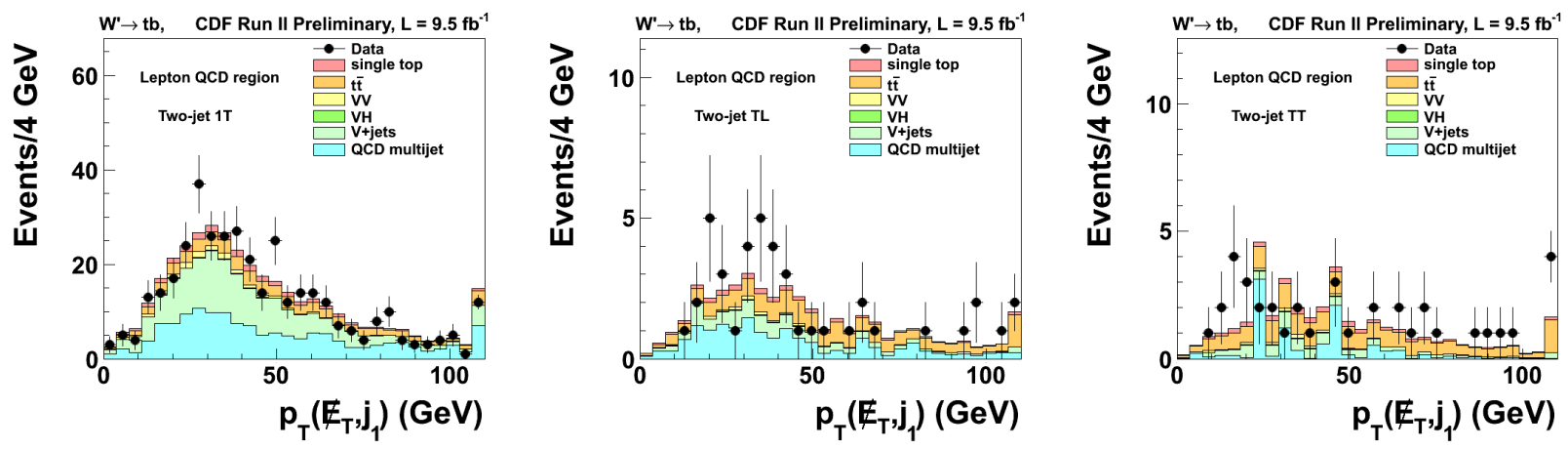

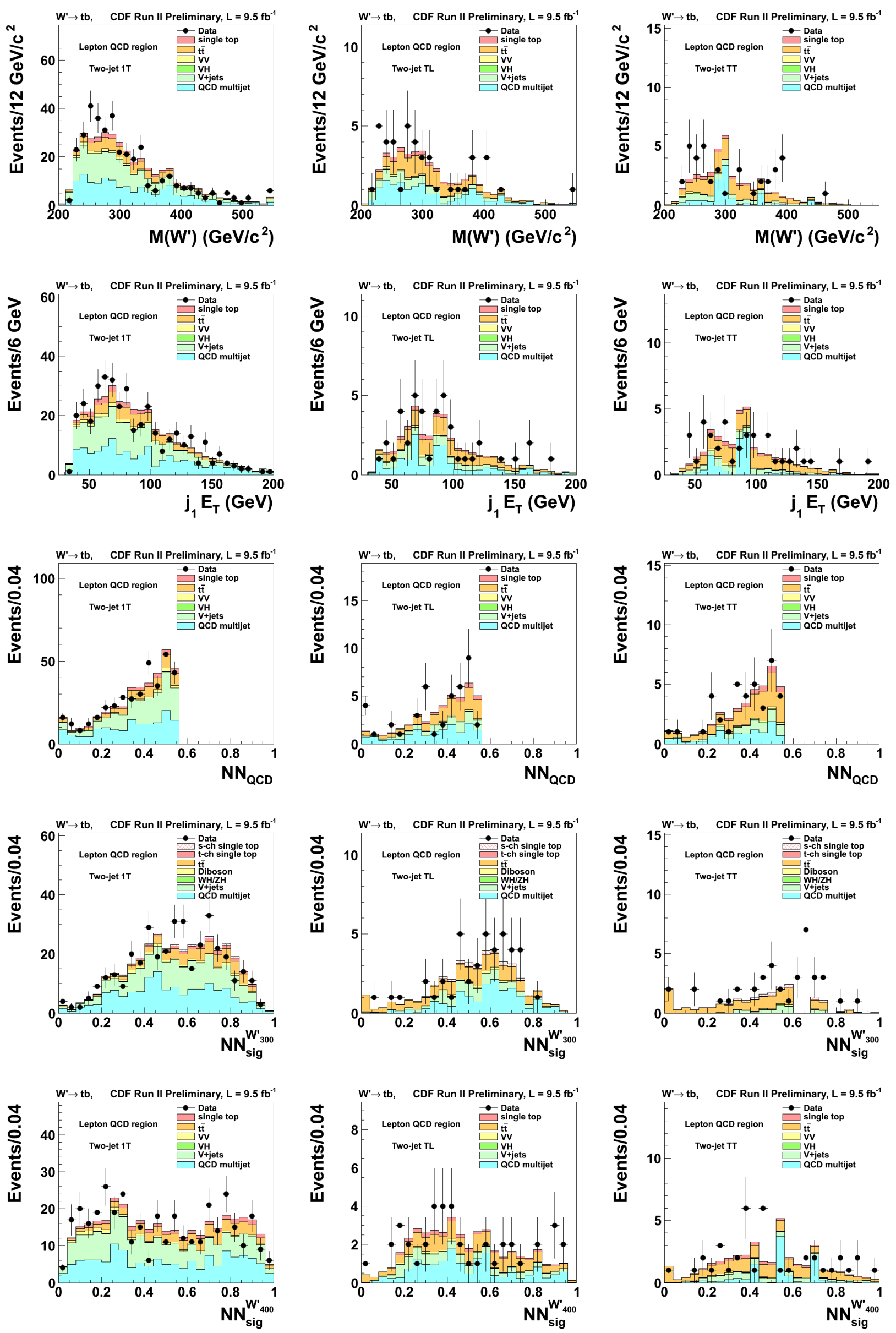

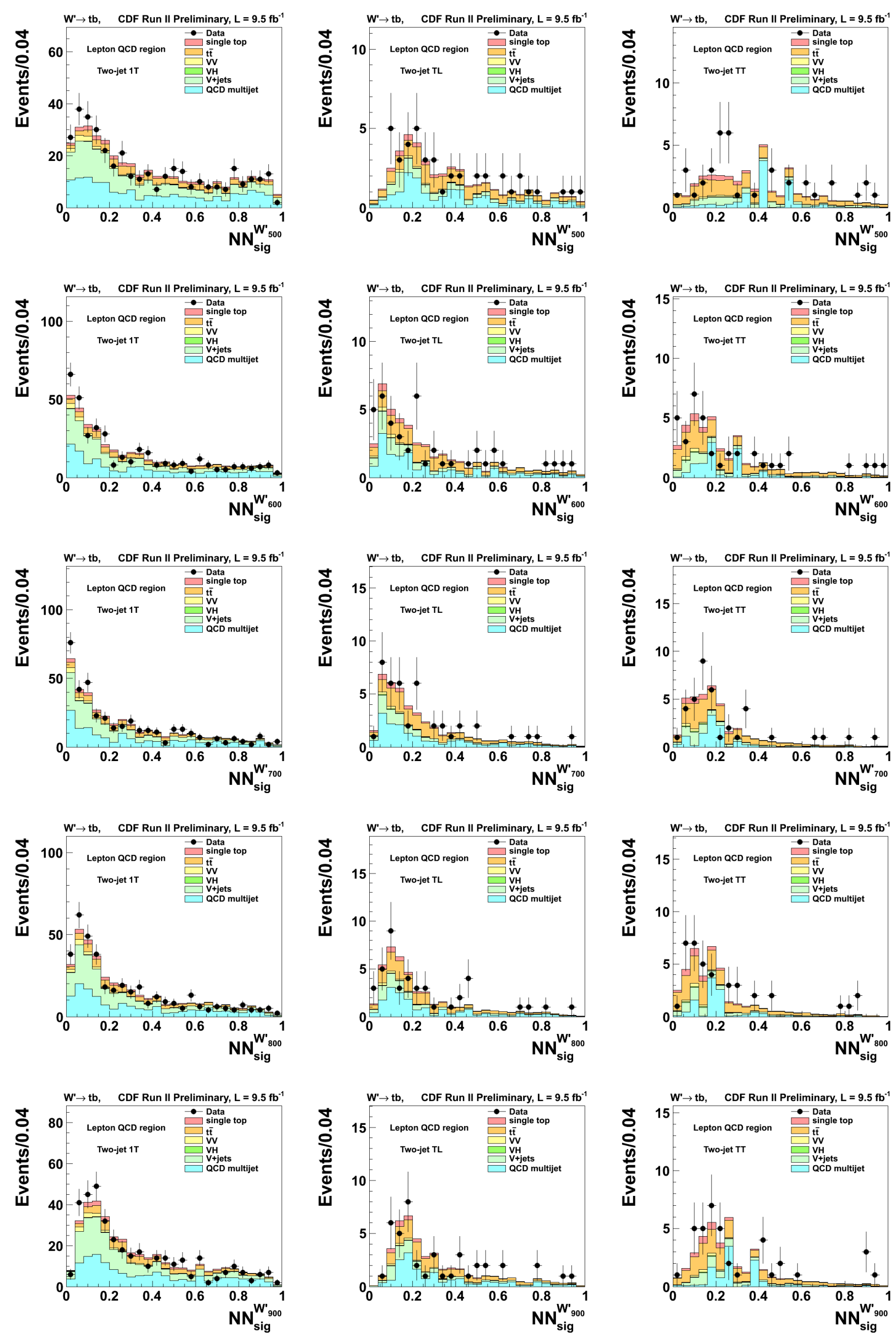


\section{C.2 Three-Jets Lepton QCD Sample}
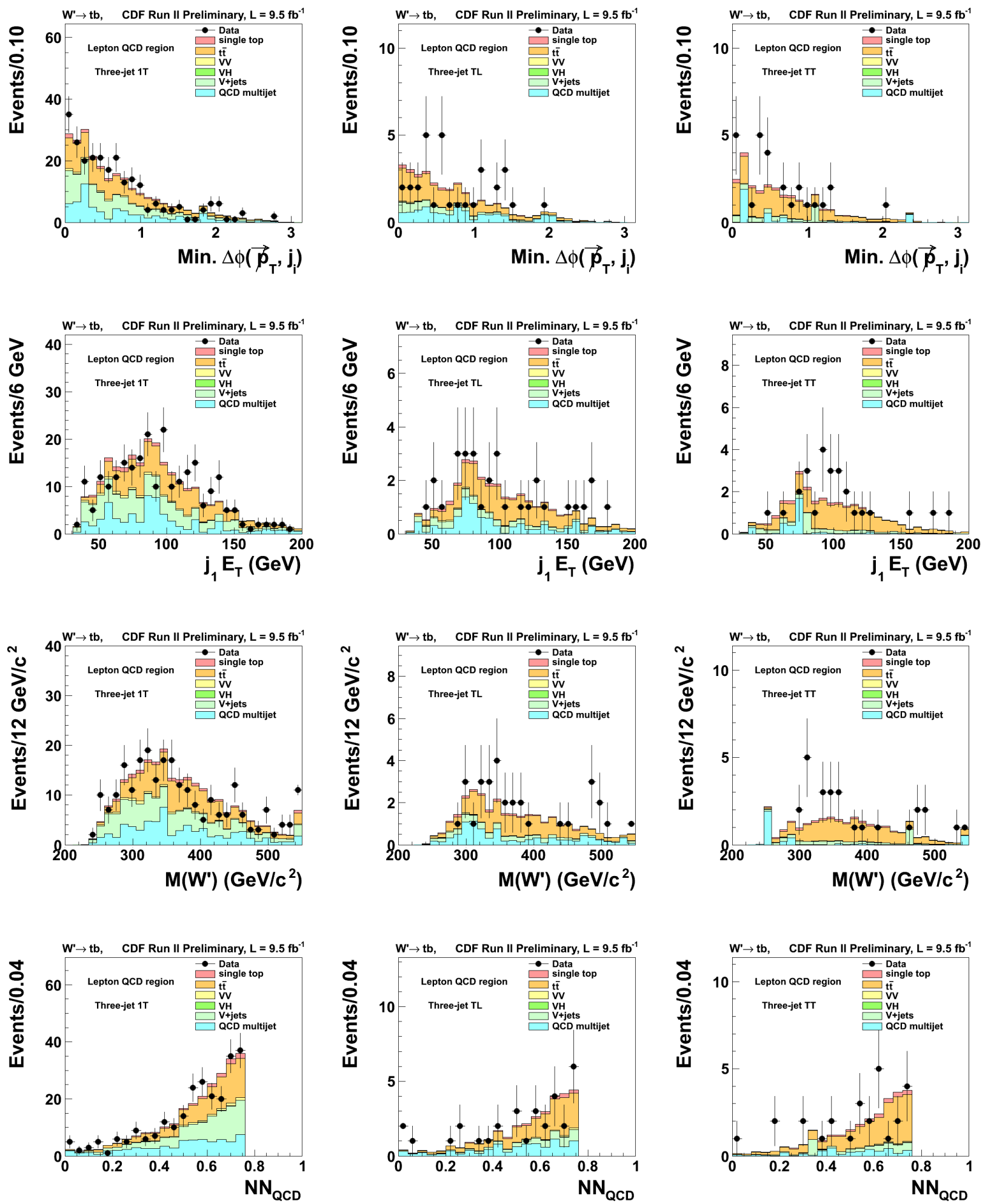

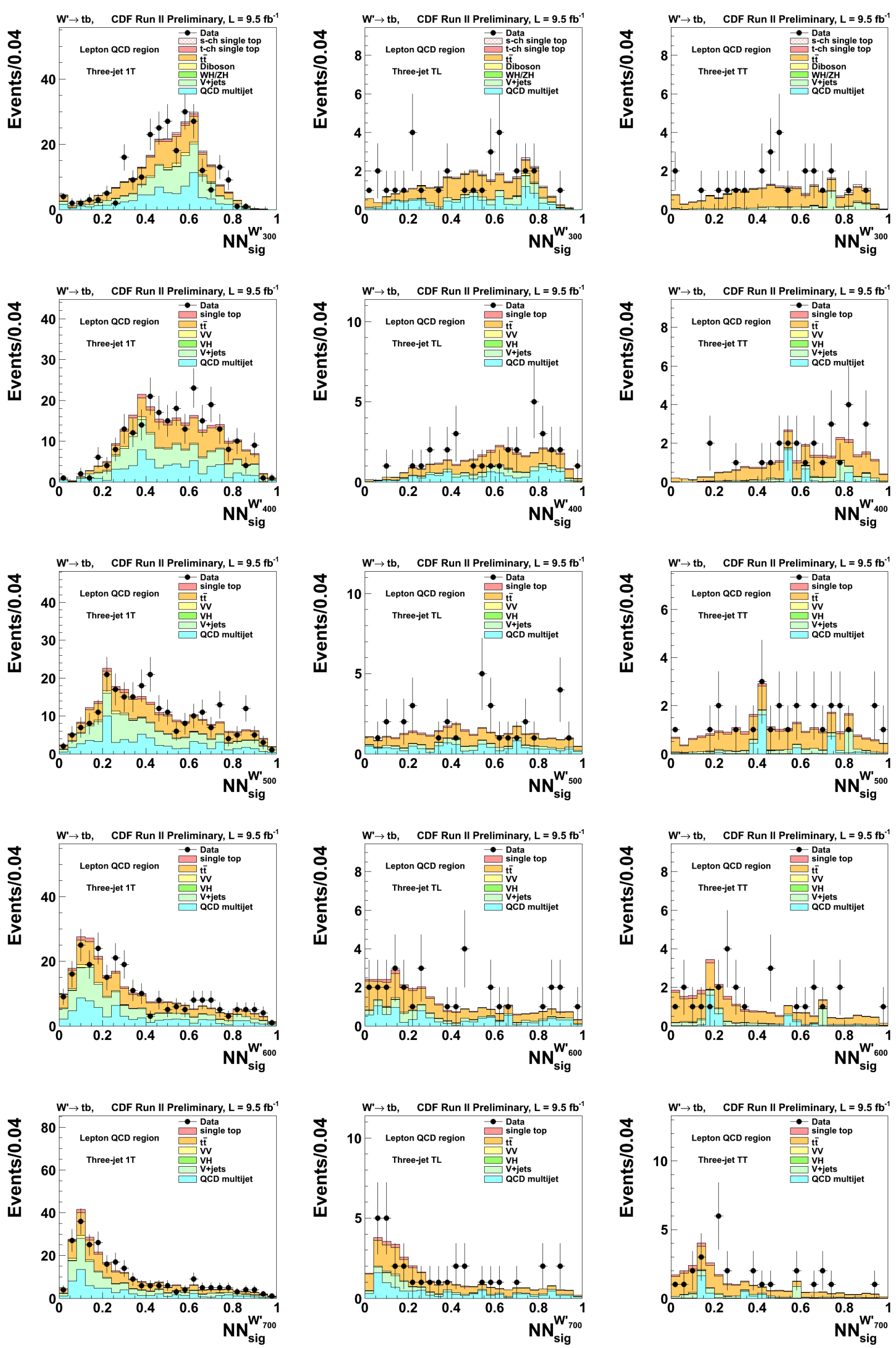

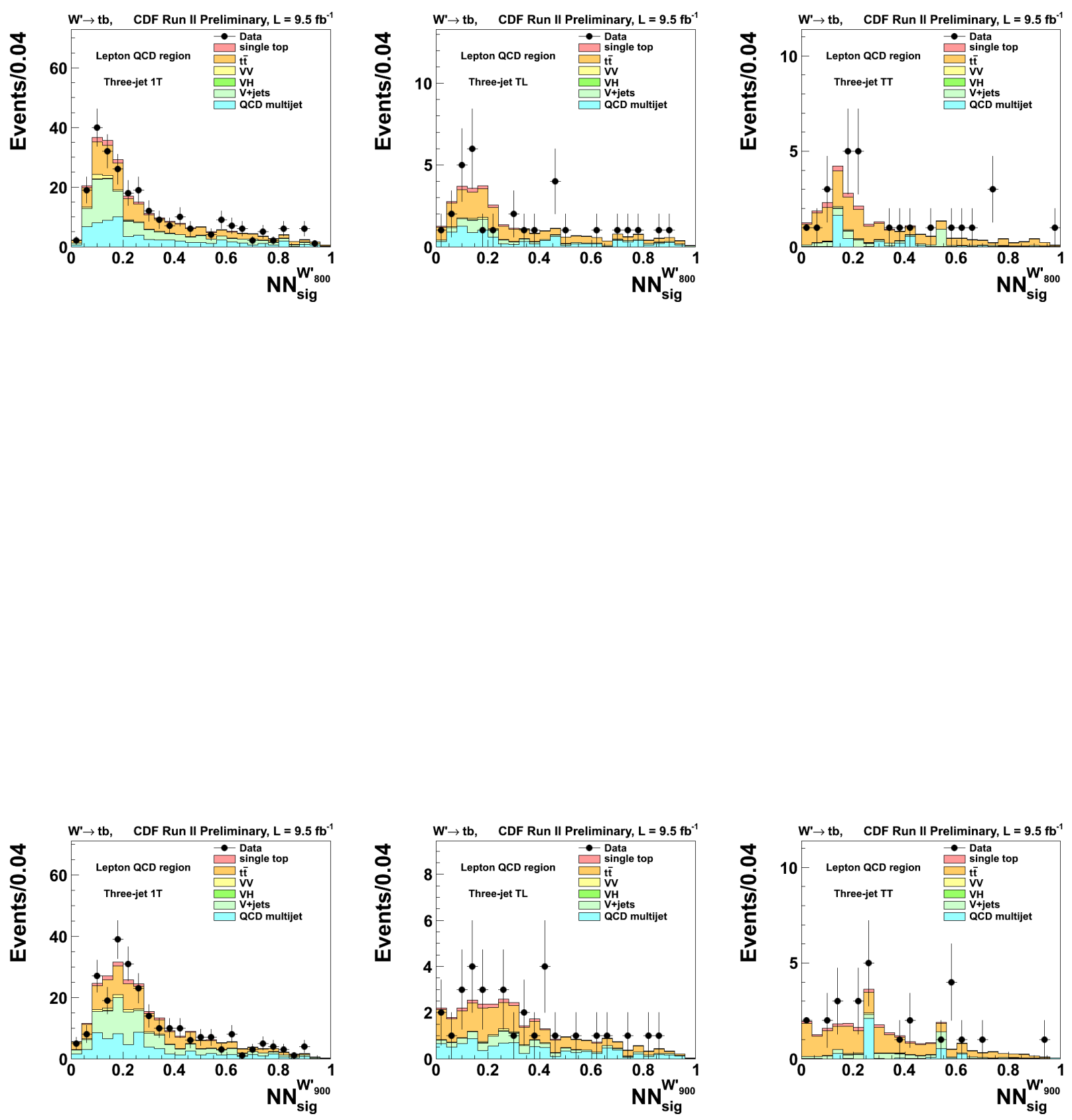


\section{C.3 Two-Jets No-Lepton QCD Sample}
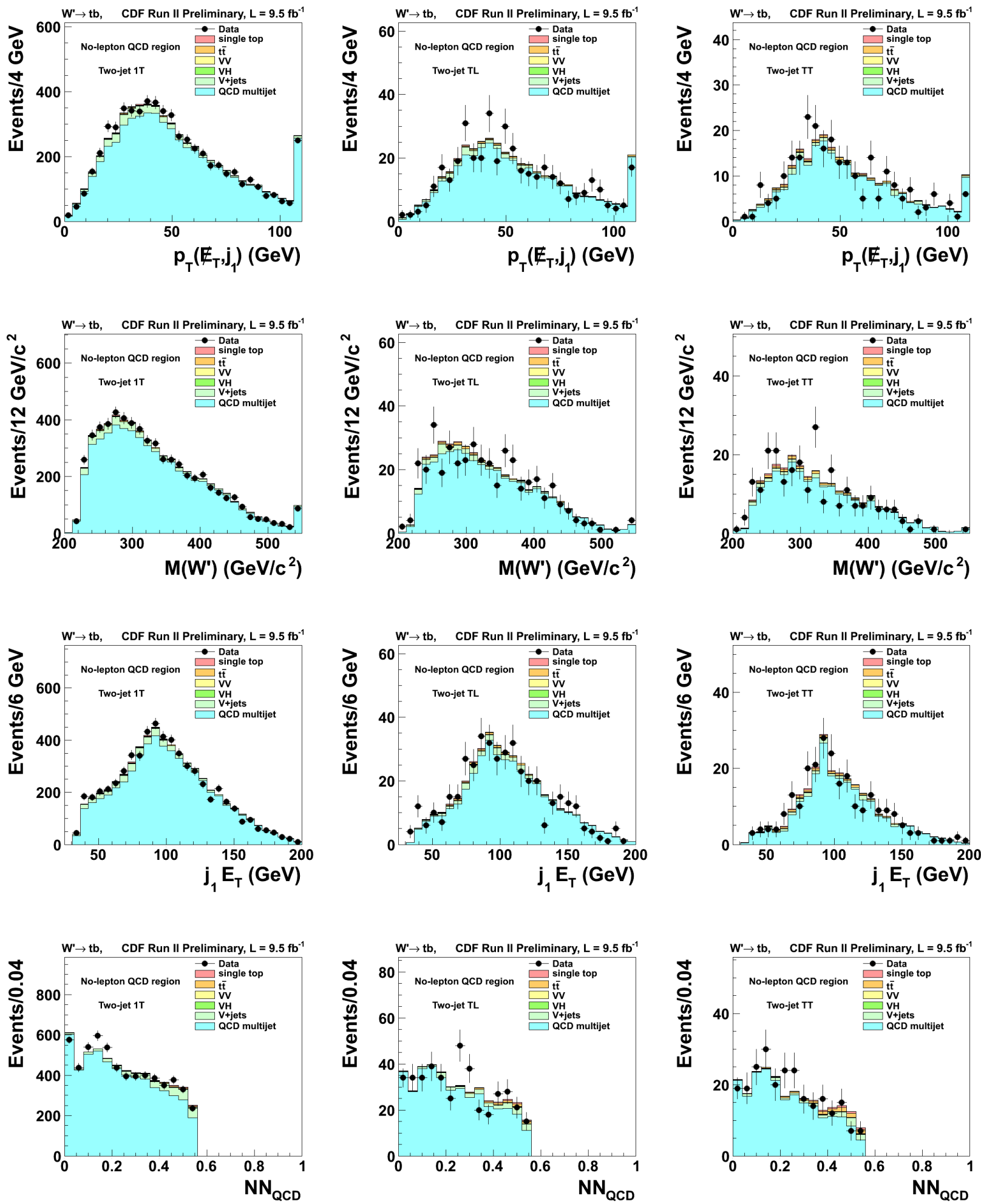

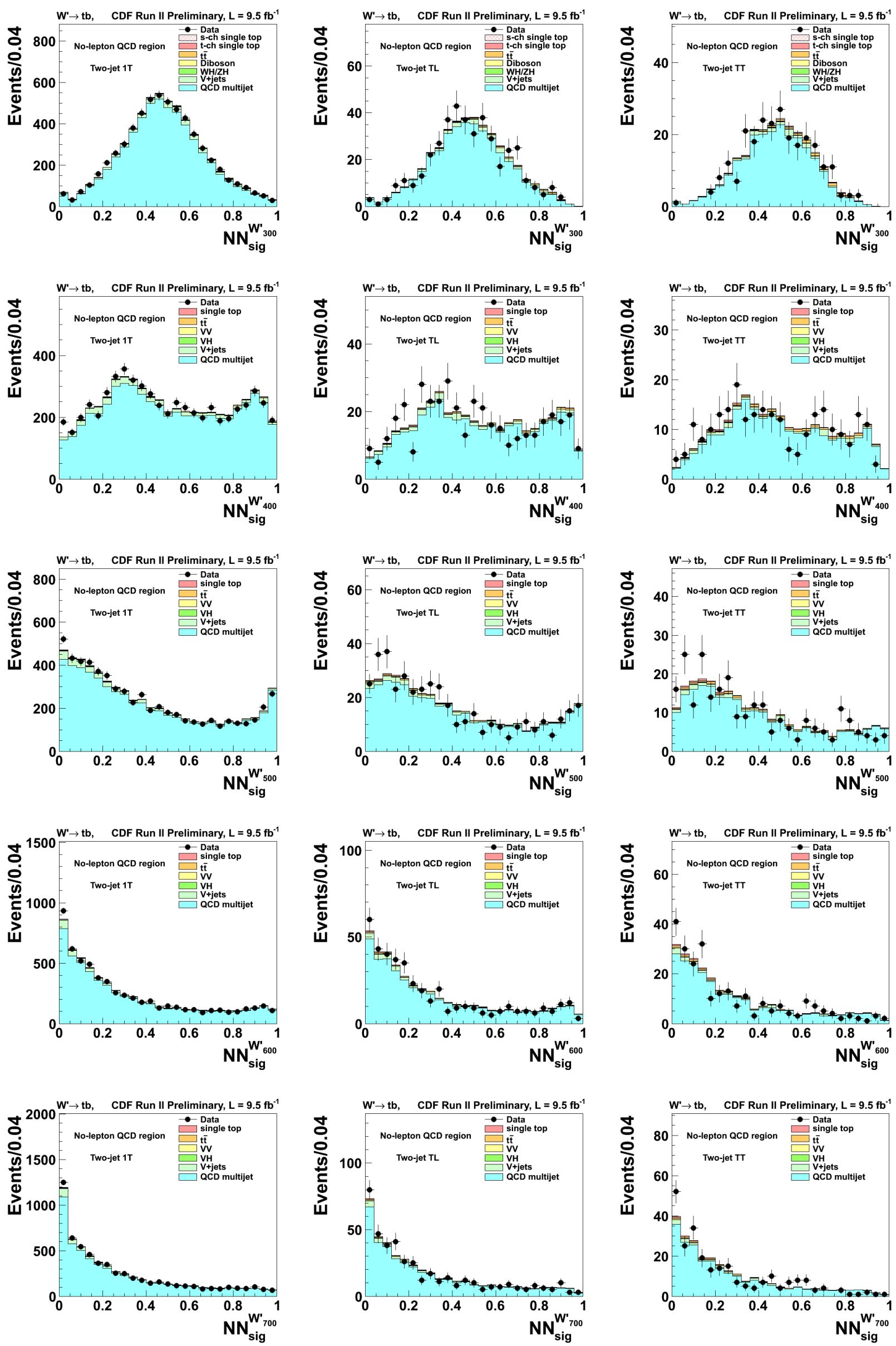

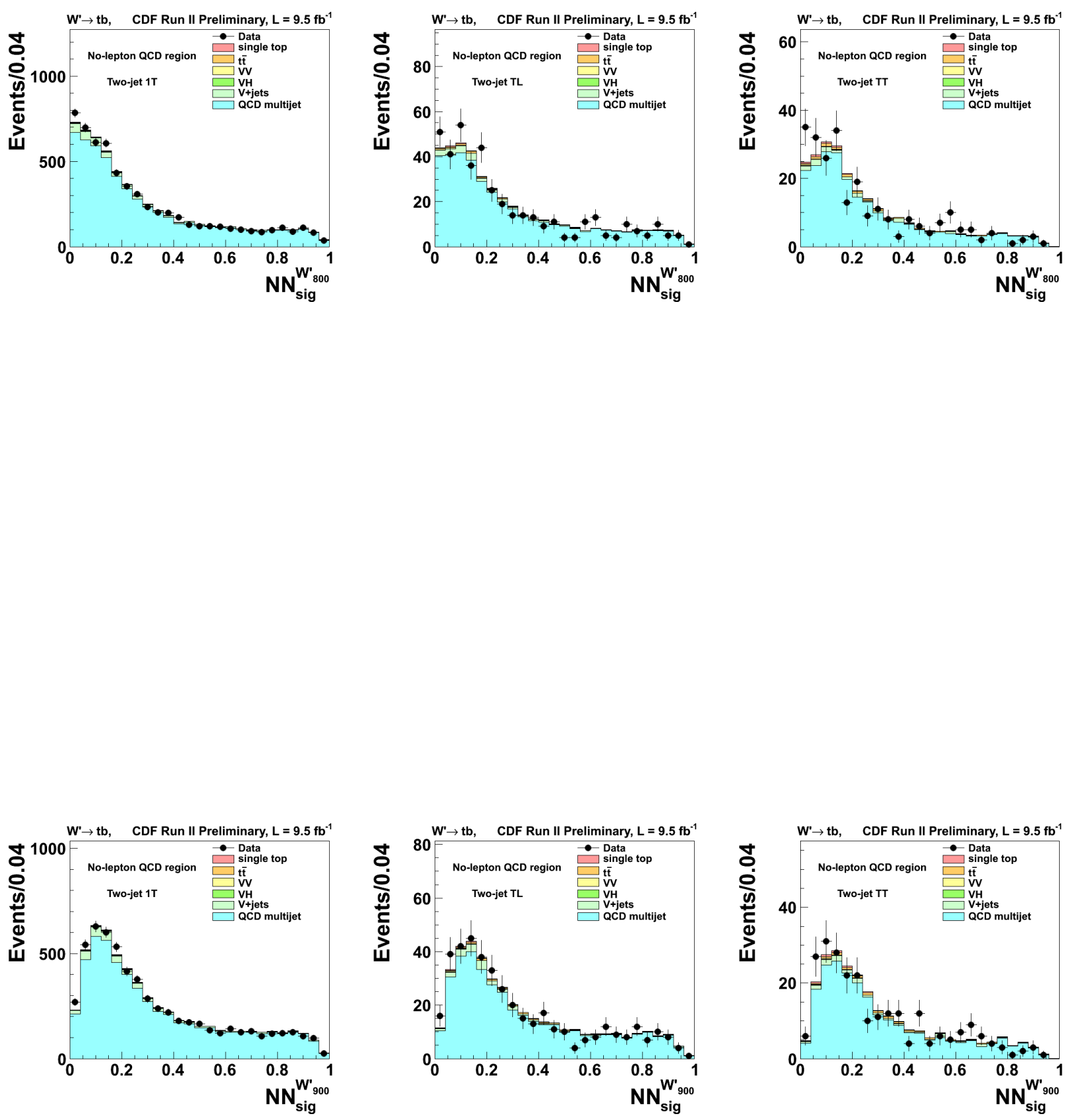


\section{C.4 Three-Jets No-Lepton QCD Sample}
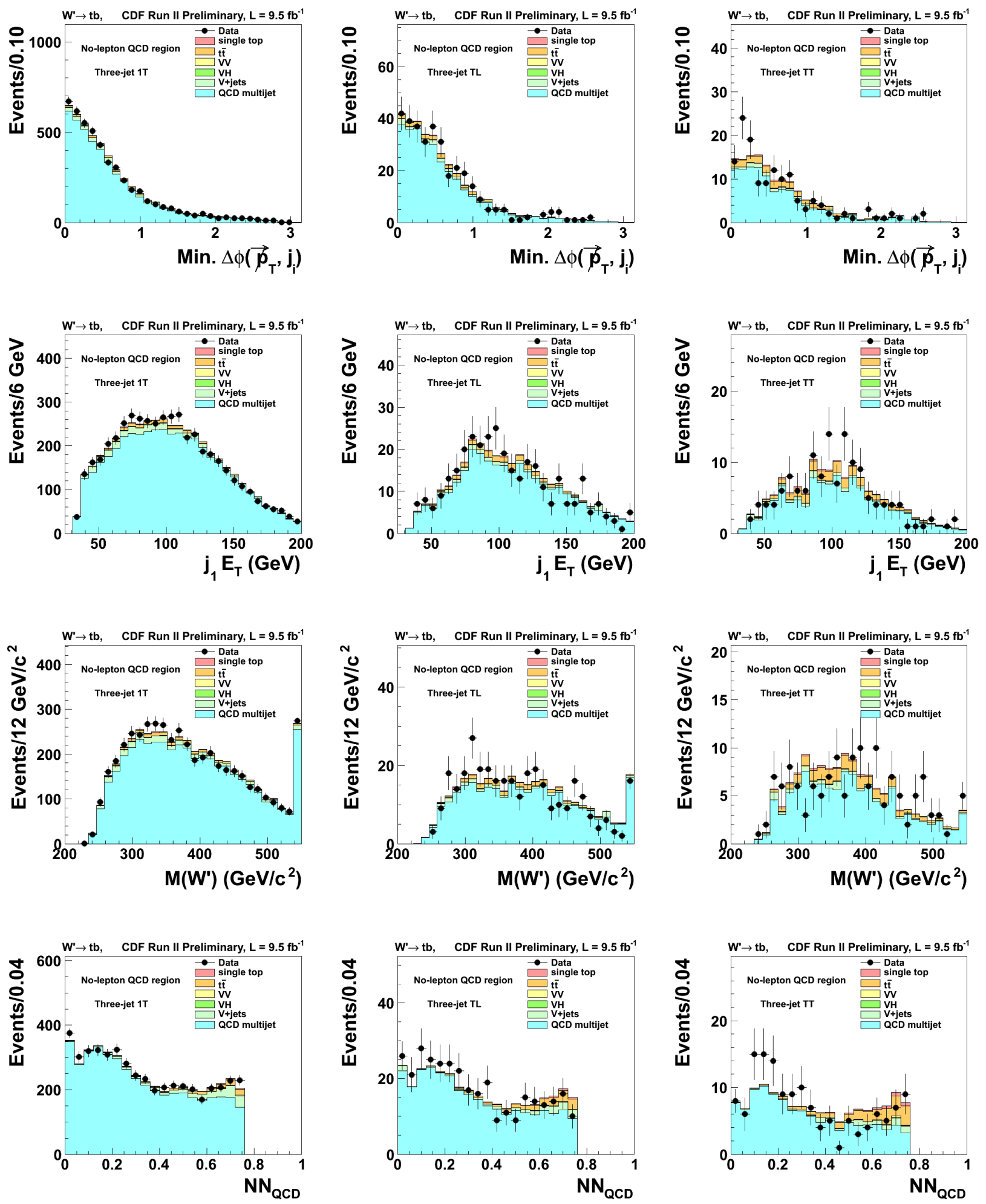

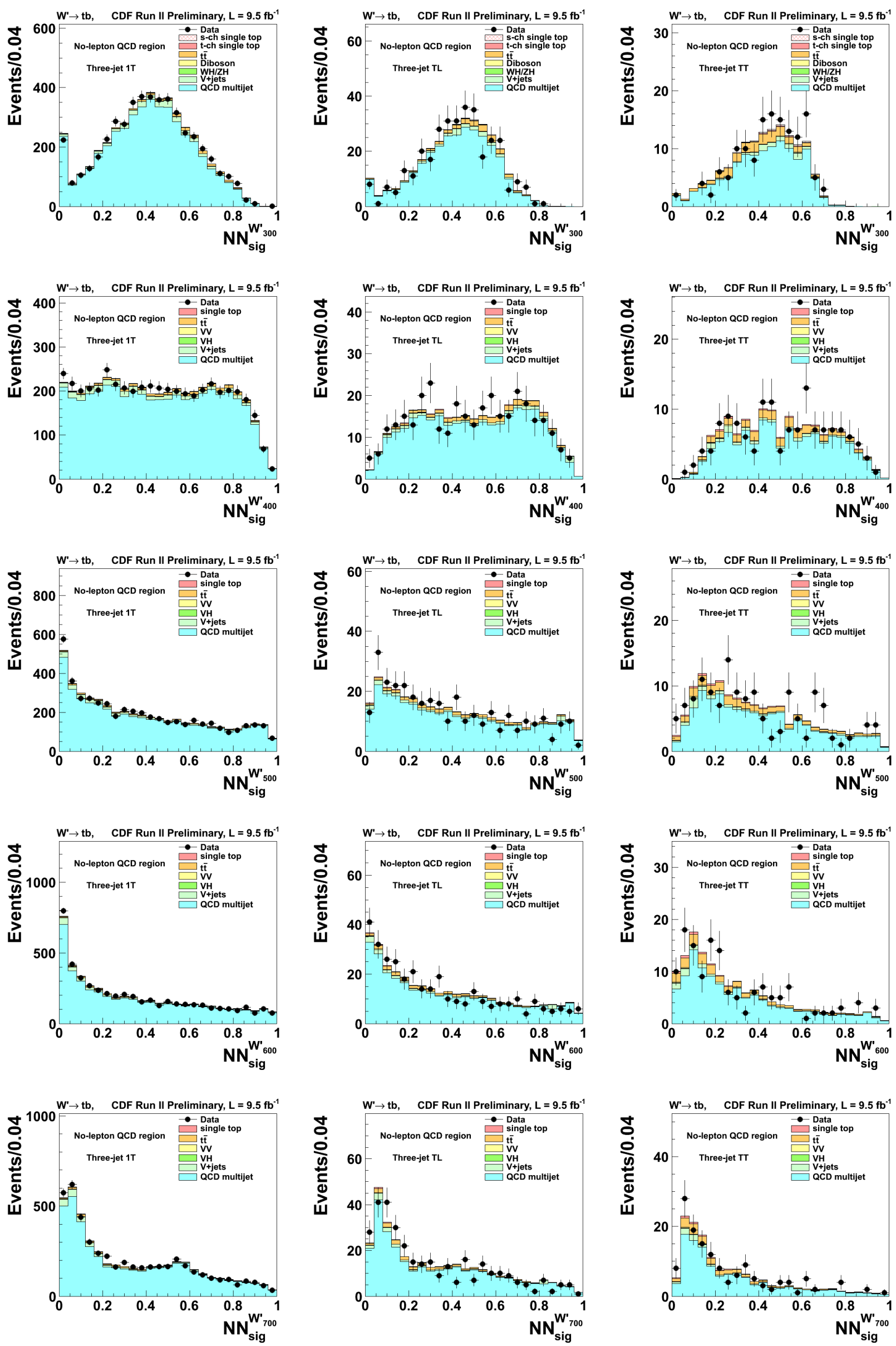

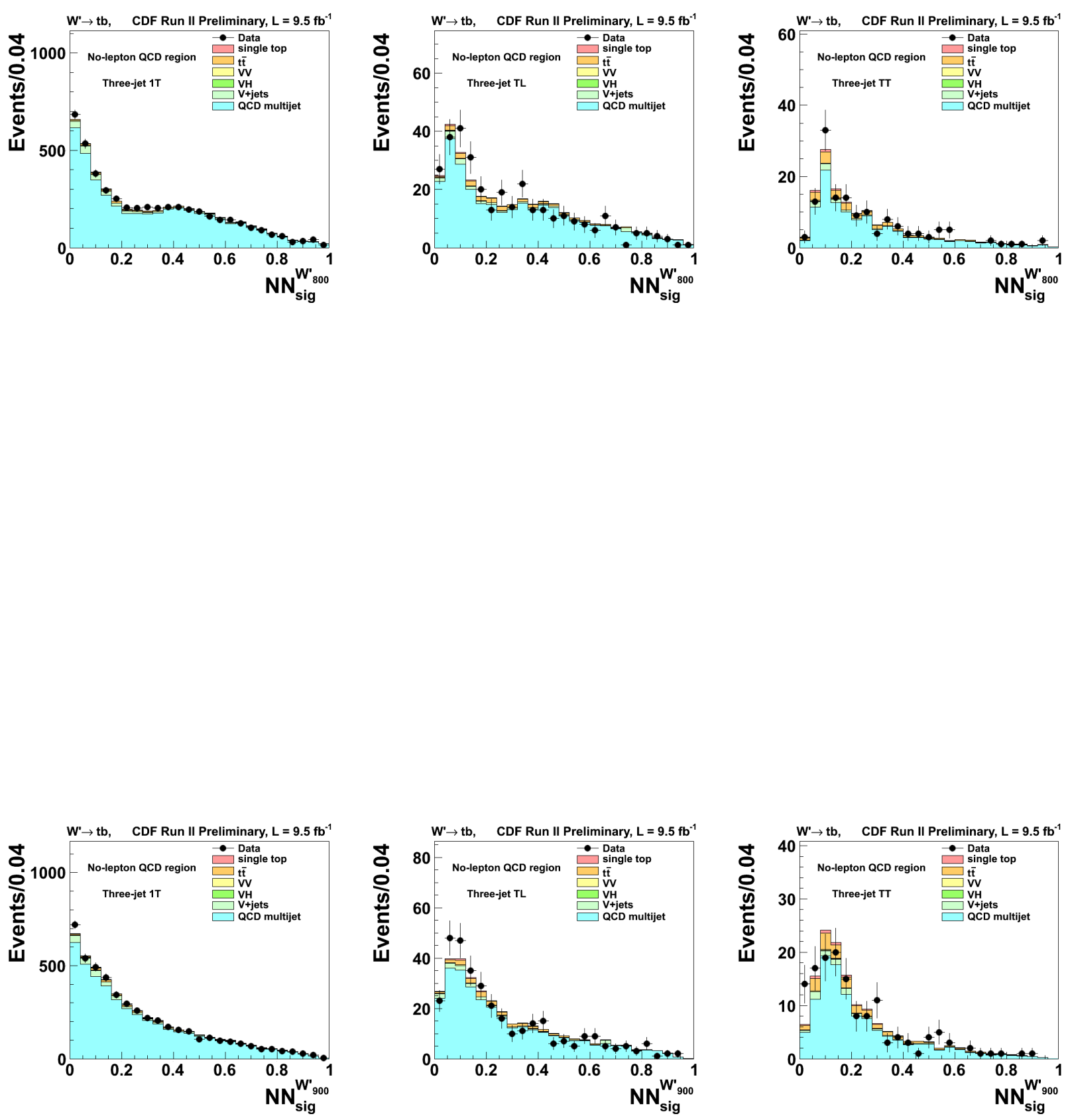


\section{Bibliography}

[1] G. Aad et al. (ATLAS Collaboration), Phys.Lett. B 716 1-29 (2012).

[2] S. Chatrchyan et al. (CMS Collaboration), Phys.Lett. B 716 30-61 (2012).

[3] G. Aad et al. (ATLAS Collaboration), Phys. Lett. B 726 (2013) 120.

[4] G. Aad et al. (ATLAS Collaboration), arXiv:hep-ex/1501.04943v2.

[5] S. L. Glashow, Nucl. Phys. 22, 579 (1961).

[6] S.F. Novaes, Standard Model: An Introduction. arXiv:hep-ph/0001283v1.

[7] P.W. Higgs, Phys. Rev. Lett. 12, 132 (1964); idem., Phys. Rev. 145, 1156 (1966); F. Englert and R. Brout, Phys. Rev. Lett. 13, 321 (1964); G.S. Guralnik, C.R. Hagen, and T.W. Kibble, Phys. Rev. Lett. 13, 585 (1964).

[8] K. Nakamura et al., J. Phys. G37, (2010) 075021.

[9] N. Cabibbo, Phys. Rev. Lett. 10, 531 (1963);

M. Kobayashi and T. Maskawa, Prog. Theor. Phys. 49, 652 (1973).

[10] I. J. R. Aitchison and A. J. G. Hey. Gauge Theories in Particle Physics 2 Volume Set. Taylor Francis, 3 edition, January 2004.

[11] F. Abe et al. (CDF Collaboration), Phys. Rev. Lett. 74, 2626 (1995);

S. Abachi et al. (D0 Collaboration), Phys. Rev. Lett. 74, 2632 (1995).

[12] The ATLAS, CDF, CMS, D0 Collaborations, arXiv:hep-ex/1403.4427v1

[13] R. Ellis, W.J. Stirling, and B.R. Webber QCD and Collider Physics Cambridge University Press 2003.

[14] S. Willenbrock, arXiv:hep-ph/0211067.

[15] A. Abulencia et al. (CDF Collaboration), Phys. Rev. D, 73, 112006 (2006).

[16] P. Baernreuther, M. Czakon and A. Mitov, Phys. Rev. Lett. 109, 132001 (2012).

[17] S. Cortese and R. Petronzio, Phys. Lett. B, 253, 494-498 (1991). 
[18] T. Stelzer and S. Willenbrock, Phys. Lett. B, 357, 125-130, (1995).

[19] M. C. Smith and S. Willenbrock, Phys. Rev. D, 54, 6696-6702 (1996).

[20] S. Mrenna and C.P. Yuan, C. P., Phys. Lett. B, 416, 1-2 (1998).

[21] A. P. Heinson, A. S. Belyaev and E. E. Boos, Phys. Rev. D, 56, 5 (1997)

[22] N. Kidonakis, Phys. Rev. D 81, 054028 (2010); 83, 091503(R) (2011).

[23] N. Kidonakis, arXiv:hep-ph/1212.2844.

[24] S. Willenbrock and D.A. Dicus, Phys. Rev. D, 34, 07 (1986).

[25] C.P. Yuan, C. P., Phys. Rev. D, 41, 42-51 (1990).

[26] R.K. Ellis and S. Parke, Phys. Rev. D, 46, 3785-3788 (1992).

[27] T. Stelzer, Z. Sullivan, and S. Willenbrock, Phys. Rev. D, 56, 11 (1997).

[28] N. Kidonakis, Phys. Rev. D, 83, 091503 (2011).

[29] N. Kidonakis, Phys. Rev. D 74, 114012 (2006).

[30] N. Kidonakis, Phys. Rev. D 82, 054018 (2010).

[31] J. Beringer et al., Phys. Rev. D 86, 010001 (2012).

[32] Ahmad et al. (SNO Collaboration), Phys. Rev. Lett. 89, 011301 (2002).

[33] Y. Fukuda. et al., Phys. Rev. Lett. 81, 1562 (1998).

[34] T. Aaltonen et al. (CDF Collaboration), Phys. Rev. Lett. 110, 121802 (2013).

[35] R. M. Harris and S. Jain, Eur. Phys. J. C 72, 2072 (2012).

[36] http://www-cdf.fnal.gov/physics/new/top/2012/MttLJwebPub/

[37] T. Aaltonen et al. (CDF Collaboration), Phys. Rev. Lett. 103, 041801 (2009)

[38] Y. Mimura and S. Nandi, Phys. Lett. B 538, 406 (2002); G. Burdman, B. Dobrescu and E. Ponton, Phys. Rev. D 74, 075008 (2006).

[39] E. Malkawi, T. Tait and C.P. Yuan, Phys. Lett. 385, 304 (1996); H. Georgi, E. Jenkins and E. Simmons, Nucl. Phys. B 331, 541 (1990).

[40] M. Perelstein, Prog. Part. Nucl. Phys. 58, (2007).

[41] J.C. Pati and A. Salam, Phys. Rev. D 10, 275 (1974); R.N. Mohapatra and J.C. Pati, Phys. Rev. D 11, 566 (1975); G. Senjanovic and R.N. Mohapatra, Phys. Rev. D 12, 1502 (1975). 
[42] D. Mohl, Phys. Reports, 58, (1980).

[43] P. C. Bhat, W. J. Spalding, arXiv:hep-ex/0410046v1.

[44] R. Blair et al., FERMILAB-DESIGN-1996-01.

[45] C. S. Hill., Nucl. Instrum. Meth. A 530, 1-6, 2004.

[46] A. Sill., Nucl. Instrum. Meth. A 447, 1-8, 2000.

[47] A. Affolder et al. (CDF Collaboration), Nucl. Instrum. Meth. A 453, 84-88, 2000.

[48] F. D. Snider, Nucl. Instrum. Methods Phys. Res., Sect. A 566, 133-141 (2006).

[49] B. Ashmanskas et al., Nucl. Instrum. Methods Phys. Res., Sect. A 518, 532-536 (2004).

[50] T. Affolder et al. (CDF Collaboration), Nucl. Instrum. Methods Phys. Res., Sect. A 526, 249-299 (2004).

[51] A. Kotwal and C. Hays, Nucl. Instrum. Methods Phys. Res., Sect. A 762, 85-99 (2014).

[52] D. Acosta et al. (CDF Collaboration), Nucl. Instrum. Methods Phys. Res., Sect. A 518, 605 (2004).

[53] C. M. Ginsburg, Eur. Phys. J. C 33, 1002-1004 (2004).

[54] G. Ascoli et al., FERMILAB-PUB-87-188-E.

[55] E. Thomson et al., IEEE Trans.Nucl.Sci. 49, 1063-1070 (2002).

[56] A. Bhatti et al., Nucl. Instrum. Methods Phys. Res., Sect. A 566, 375-412 (2006).

[57] C. Adloff et al., Z. Phys. C74, 221-236 (1997).

[58] T. Affolder et al., Phys. Rev. D 64, 032001 (2001).

[59] T. Affolder et al., Phys. Rev. D 63, 032003 (2001).

[60] T. Aaltonen et al., Phys. Rev. Lett. 104, 141801 (2010).

[61] D. Acosta et al., Phys. Rev. D 71, 052003 (2005).

[62] A. Abulencia et al., Phys. Rev. D 74, 072006 (2006).

[63] J. Freeman, T. Junk, M. Kirby, Y. Oksuzian, T. J. Phillips, F. D. Snider, M. Trovato, J. Vizan, and W. M. Yao, Nucl. Instrum. Methods Phys. Res., Sect. A 697, 64 (2013).

[64] P.C. Bhat, Annu. Rev. Nucl. Part. Sci. 2011. 61:281309

[65] C. Ferrazza, Master's thesis, Universita "La Sapienza" Roma (2006).

P. Mastrandrea, FERMILAB-THESIS-2008-63. 
[66] J. Freeman et al., Nucl. Instrum. Methods Phys. Res., Sect. A 663, 27-37 (2012).

[67] D. Acosta et al., CDF Note 7410.

[68] T. Aaltonen et al. (CDF Collaboration), Phys. Rev. Lett. 103, 092002 (2009).

[69] J. Antos et al., IEEE Trans. Nucl. Sci. 53, 2897-2906 (2006).

[70] S. Klimenko, J. Konigsberg and T. Liss, Fermilab-FN-0741 (2003).

[71] A. Bhatti et al., IEEE Trans. Nucl. Sci. 56, 3 (2009).

[72] O. Gonzalez, K. Potamianos, M. Vidal and H. Wolfe, CDF note 10538.

K. Potamianos, Ph.D. Thesis, Purdue University, FERMILAB-THESIS-2011-34 (2011).

[73] M.L. Mangano, M. Moretti, F. Piccinini, R. Pittau, and A.D. Polosa, J. High Energy Phys. 0307 (2003) 001.

[74] T. Sjostrand, S. Mrenna, and P. Skands, J. High Energy Phys. 05 (2006) 026.

[75] M. L. Mangano, M. Moretti, F. Piccinini, and M. Treccani, J. High Energy Phys. 01 (2007) 013.

[76] J. Alwall, S. Hoche, F. Krauss, N. Lavesson, L. Lonnblad, F. Maltoni, M. Mangano, M. Moretti, C. Papadopoulos, F. Piccinini, S. Schumann, M. Treccani, J. Winter, and M. Worek, Eur. Phys. J. C 53, 473 (2008).

[77] T. Aaltonen et al. (CDF Collaboration), Phys. Rev. Lett. 110, 071801 (2013).

[78] T. Aaltonen et al. (CDF Collaboration), Phys. Rev. D 82, 112005 (2010).

[79] J. M. Campbell and R. K. Ellis, Phys. Rev. D 60, 113006 (1999).

[80] J. Baglio and A. Djouadi, J. High Energy Phys. 10 (2010) 064;

O. Brien, R. V. Harlander, M. Weisemann, and T. Zirke, Eur. Phys. J. C 72, 1868 (2012).

[81] S. Alioli, P. Nason, C. Oleari, and E. Re, J. High Energy Phys. 06 (2010) 043.

[82] H. Lai, J. Huston, S. Kuhlmann, J. Morfin, F. Olness, J. Owens, J. Pumplin, and W. Tung, Eur. Phys. J. C 12, 375 (2000).

[83] T. Aaltonen et al. (CDF Collaboration), Phys. Rev. D 82, 034001 (2010).

[84] GEANT, detector description and simulation tool, CERN Program Library Long Writeup W5013 (1993).

[85] G. Grindhammer and S. Peters, arxiv:hep-ex/001020.

[86] D. Acosta et al. (CDF Collaboration), Phys. Rev. D 71, 052003 (2005);

A. Abulencia et al. (CDF Collaboration), Phys. Rev. D 74, 072006 (2006). 
[87] E. Boos et al., Phys. Lett. B 655, 245 (2007).

[88] Mr. Bayes and Mr. Price, An essay towards solving a problem in the doctrine of chances. by the late rev. mr. bayes, f. r. s. communicated by mr. price, in a letter to john canton, a. m. f. r. s. Philosophical Transactions, 53:370-418, 1763.

[89] Statistics, in K. Nakamura et al. (Particle Data Group), J. Phys. G 37075021 (2010).

[90] N. Metropolis, A. W. Rosenbluth, M. N. Rosenbluth, A. H. Teller, and E. Teller, Journal of Chemical Physics, 21:1087-1092 (1953);

W.K. Hastings, Biometrika, 97-109 (1970).

[91] T. Junk., Nucl. Instrum. Methods Phys. Res., Sect. A 434 , 435-443 (1999).

T. Junk., CDF/DOC/STATISTICS/PUBLIC / 8128.

[92] G. Cowan, K. Cranmer, E. Gross, and O. Vitells, Eur. Phys. J. C 71, 1554 (2011).

[93] V. M. Abazov et al. (D0 Collaboration), Phys. Rev. Lett. 103, 092001 (2009).

[94] CDF and D0 Collaboration, Tevatron Electroweak Working Group, arXiv:hepex/0908.2171.

[95] V. Abazov et al. (D0 Collaboration), Phys. Lett. B 705, 313 (2011).

[96] G. Aad et al. (ATLAS Collaboration), Phys. Lett. B 717, 330 (2012).

[97] S. Chatrchyan et al. (CMS Collaboration), J. High Energy Phys. 12 (2012) 035.

[98] V. M. Abazov et al. (D0 Collaboration), Phys. Lett. B 726, 656 (2013).

[99] T. Aaltonen et al. (CDF Collaboration), Phys. Rev. Lett. 112, 231804 (2014).

[100] T. Aaltonen et al. (CDF Collaboration), Phys. Rev. D 87, 052008 (2013).

[101] M. Kirby, private communications.

[102] M. Bentivegna, Q. Liu, F. Margaroli, and K. Potamianos, arxiv:hep-ex/1205.4470.

[103] V. M. Abazov et al. (D0 Collaboration), Nucl. Instrum. Methods A 620, 490 (2010);

V. M. Abazov et al. (D0 Collaboration), arXiv:hep-ex/1312.7623.

[104] V. M. Abazov et al. (D0 Collaboration), Phys. Lett. B 699, 145 (2011).

[105] G. Aad et al. (ATLAS Collaboration), 2014), arXiv:hep-ex/1410.4103.

[106] S. Chatrchyan et al. (CMS Collaboration), J. High Energy Phys. 05 (2014) 108.

[107] Z. Sullivan, Phys. Rev. D 66, 075011 (2002).

[108] T. Aaltonen et al. (CDF Collaboration), Phys. Rev. D 81, 072003 (2010).

[109] T. Aaltonen et al. (CDF Collaboration), arXiv:hep-ex/1407.4031. 